\title{
Constant mean curvature spheres in homogeneous three-manifolds
}

\author{
William H. Meeks III ${ }^{1}$ • Pablo Mira ${ }^{2}$. \\ Joaquín Pérez ${ }^{3}$. Antonio Ros ${ }^{3}$
}

Received: 9 December 2017 / Accepted: 16 September 2020 /

Published online: 30 October 2020

(C) The Author(s) 2020

\begin{abstract}
We prove that two spheres of the same constant mean curvature in an arbitrary homogeneous three-manifold only differ by an ambient isometry, and we determine the values of the mean curvature for which such spheres exist.
\end{abstract}

William H. Meeks III: This material is based upon work for the NSF under Award No.

DMS - 1309236. Any opinions, findings, and conclusions or recommendations expressed in this publication are those of the authors and do not necessarily reflect the views of the NSF. Pablo Mir was partially supported by MINECO/FEDER, Grant No. MTM2016-80313-P, and Programa de Apoyo a la Investigacion, Fundacion Seneca-Agencia de Ciencia y Tecnologia Region de Murcia, 19461/PI/14. Research of the Joaquín Pérez and Antonio Ros partially supported by MINECO/FEDER Grants Nos. MTM2014-52368-P and MTM2017-89677-P, and Regional J. Andalucia Grant no. P18-FR-4049.

$凶$ Joaquín Pérez

jperez@ugr.es

William H. Meeks III

profmeeks@gmail.com

Pablo Mira

pablo.mira@upct.es

Antonio Ros

aros@ugr.es

1 Mathematics Department, University of Massachusetts, Amherst, MA 01003, USA

2 Departamento de Matemática Aplicada y Estadística, Universidad Politécnica de Cartagena, 30203 Cartagena, Murcia, Spain

3 Department of Geometry and Topology and Institute of Mathematics (IEMath-GR), University of Granada, 18071 Granada, Spain 
This gives a complete classification of immersed constant mean curvature spheres in three-dimensional homogeneous manifolds.

Mathematics Subject Classification Primary 53A10; Secondary 49Q05 . $53 \mathrm{C} 42$

\section{Contents}

1 Introduction . . . . . . . . . . . . . . . . . . . . . . 148

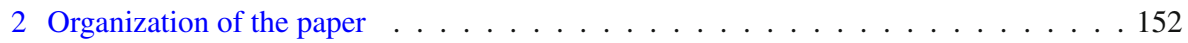

3 Reduction of Theorems 1.1 and 1.2 to Theorem $1.5 \ldots \ldots$. . . . . . . . . . 154

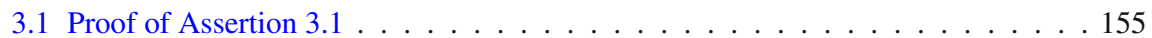

3.2 Proof of Assertion $3.2 \ldots \ldots$. . . . . . . . . . . . . . . . . . 164

3.3 Constant mean curvature spheres in complete locally homogeneous three-manifolds 165

4 Background on constant mean curvature surface theory in metric Lie groups . . . . 166

4.1 Invariant constant mean curvature surfaces . . . . . . . . . . . . . . . . . . 166

4.2 Metric semidirect products . . . . . . . . . . . . . . . . . . . . . . . . . . . . . . . . . . . . .

4.3 Metric Lie groups isomorphic to $\widetilde{\mathrm{SL}}(2, \mathbb{R}) \ldots \ldots . \ldots . \ldots 171$

5 The geometry of invariant limit surfaces of index-one spheres . . . . . . . . . . 178

6 The existence of periodic invariant limit surfaces . . . . . . . . . . . . . . 196

7 Proof of Theorem 1.5 when $X$ is a semidirect product . . . . . . . . . . . . . 206

7.1 Proof of Theorem 7.2 when $K_{\Sigma}$ is horizontal $\ldots \ldots \ldots$. . . . . . . . . 208

7.2 Case (S2.3) is impossible when $K_{\Sigma}$ is not horizontal . . . . . . . . . . . . 209

7.3 Case (S2.2) is impossible when $K_{\Sigma}$ is not horizontal . . . . . . . . . . . 210

7.4 Case (S2.1) is impossible when $K_{\Sigma}$ is not horizontal . . . . . . . . . . . . 217

8 Proof of Theorem 1.5 when $X$ is isomorphic to $\widetilde{\mathrm{SL}}(2, \mathbb{R}) \ldots \ldots . \ldots 221$

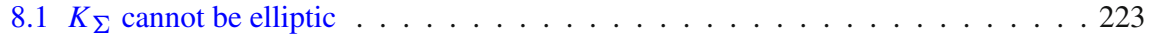

8.2 Proof of Theorem 8.1 when $K_{\Sigma}$ is parabolic . . . . . . . . . . . . . . . 224

8.3 Proof of Theorem 8.1 when $K_{\Sigma}$ is hyperbolic and case (S2.3) holds . . . . . 227

8.4 Case (S2.2) is impossible if $K_{\Sigma}$ is hyperbolic . . . . . . . . . . . . . 228

8.5 Case (S2.1) is impossible if $K_{\Sigma}$ is hyperbolic . . . . . . . . . . . . 233

9 Appendix: A nonvanishing result for the CMC flux . . . . . . . . . . . . . 235

References . . . . . . . . . . . . . . . . . . . . . . . . . . . . 242

\section{Introduction}

In this manuscript we solve the fundamental problem of classifying constant mean curvature spheres in an arbitrary homogeneous three-manifold, where by a sphere, we mean a closed immersed surface of genus zero:

Theorem 1.1 Let $M$ be a Riemannian homogeneous three-manifold. Then, any two spheres in $M$ of the same constant mean curvature differ by an isometry of $M$. Moreover, if $X$ denotes the Riemannian universal cover of $M$, and $\mathrm{Ch}(X)$ denotes the Cheeger constant of $X$, then:

1. If $X$ is not diffeomorphic to $\mathbb{R}^{3}$, then, for every $H \in \mathbb{R}$, there exists a sphere of constant mean curvature $H$ in $M$. 
2. If $X$ is diffeomorphic to $\mathbb{R}^{3}$, then the values $H \in \mathbb{R}$ for which there exists a sphere of constant mean curvature $H$ in $M$ are exactly those with $|H|>$ $\mathrm{Ch}(X) / 2$.

Our study of constant mean curvature spheres in homogeneous threemanifolds provides a natural parameterization of their moduli space and fundamental information about their geometry and symmetry, as explained in the next theorem.

Theorem 1.2 Let $M$ be a Riemannian homogeneous three-manifold, $X$ denote its Riemannian universal cover and let $S_{H}$ be a sphere of constant mean curvature $H \in \mathbb{R}$ in $M$. Then:

1. $S_{H}$ is maximally symmetric; that is, there exists a point $p \in M$, which we call the center of symmetry of $S_{H}$, with the property that any isometry $\Phi$ of $M$ that fixes $p$ also satisfies $\Phi\left(S_{H}\right)=S_{H}$. In particular, constant mean curvature spheres are totally umbilical if $M$ has constant sectional curvature, and are spheres of revolution if $M$ is rotationally symmetric.

2. If $H=0$ and $X$ is a Riemannian product $\mathbb{S}^{2}(\kappa) \times \mathbb{R}$, where $\mathbb{S}^{2}(\kappa)$ is a sphere of constant curvature $\kappa>0$, then it is well-known that $S_{H}$ is totally geodesic, stable and has nullity 1 for its stability operator. Otherwise, $S_{H}$ has index 1 and nullity 3 for its stability operator, and it is Alexandrov embedded; that is, the immersion of $S_{H}$ into $M$ extends to an isometric immersion $F: B \rightarrow M$ of a Riemannian topological 3-ball $B$, such that $\partial B=S_{H}$ is mean convex, i.e. the mean curvature of $\partial B$ with respect to its inward pointing normal vector is nonnegative.

Moreover, if $\mathcal{M}_{M}(p)$ denotes the space of spheres of nonnegative constant mean curvature in $M$ that have a base point $p \in M$ as a center of symmetry, then the map $S_{H} \in \mathcal{M}_{M}(p) \mapsto H \in \mathbb{R}$ that assigns to each sphere $S_{H}$ its mean curvature is a homeomorphism between: $(i) \mathcal{M}_{M}(p)$ and $[0, \infty)$ if $X$ is not diffeomorphic to $\mathbb{R}^{3}$, or (ii) $\mathcal{M}_{M}(p)$ and $(\mathrm{Ch}(X) / 2, \infty)$ if $X$ is diffeomorphic to $\mathbb{R}^{3}$. This homeomorphism is real analytic except at $H=0$ when $X=\mathbb{S}^{2}(\kappa) \times \mathbb{R}$.

Theorems 1.1 and 1.2 can be generalized to the case where $M$ is a complete, locally homogeneous three-manifold; see Sect. 3.3.

Remark 1.3 The Cheeger constant $\operatorname{Ch}(Y)$ of a Riemannian manifold $Y$ with infinite volume is defined as the infimum of the quotients $\frac{\operatorname{Area}(\partial D)}{\operatorname{Volume}(D)}$ where $D$ is any smooth compact domain in $Y$. When $Y=X$ is a homogeneous manifold diffeomorphic to $\mathbb{R}^{3}$, the value of $\mathrm{Ch}(X)$ is explicitly known, except when $X$ is isometric to the universal cover of the special linear group $\widetilde{\mathrm{SL}}(2, \mathbb{R})$ endowed with a left invariant metric with a three-dimensional isometry group; see Section 2.2 in [27]. It is a problem of independent interest to determine the 
exact value of $\mathrm{Ch}(X)$ in such spaces $X=(\widetilde{\mathrm{SL}}(2, \mathbb{R}),\langle\rangle$,$) , which is known to$ be positive; see [27] and item 5 of Lemma 4.9 of the present paper for this and other related partial results.

The classification of constant mean curvature spheres is an old problem. In the nineteenth century, Jellet [18] proved that any constant mean curvature sphere in $\mathbb{R}^{3}$ that is star-shaped with respect to some point is a round sphere. In 1951, Hopf [16] introduced a holomorphic quadratic differential for any constant mean curvature surface in $\mathbb{R}^{3}$ and then used it to prove that constant mean curvature spheres in $\mathbb{R}^{3}$ are round. His proof also worked in the other simply-connected three-dimensional spaces of constant sectional curvature, which shows that these spheres are, again, totally umbilical (hence, they are the boundary spheres of geodesic balls of the space); see e.g. [6,7].

In 2004, Abresch and Rosenberg [1,2] proved that any constant mean curvature sphere in the product spaces $\mathbb{H}^{2}(\kappa) \times \mathbb{R}$ and $\mathbb{S}^{2}(\kappa) \times \mathbb{R}$, where $\mathbb{S}^{2}(\kappa)$ (resp. $\left.\mathbb{H}^{2}(\kappa)\right)$ denotes the two-dimensional sphere (resp. the hyperbolic plane) of constant Gaussian curvature $\kappa \neq 0$, and more generally, in any simply connected Riemannian homogeneous three-manifold with an isometry group of dimension four, is a rotational sphere. Their theorem settled an old problem posed by Hsiang and Hsiang [17], and reduced the classification of constant mean curvature spheres in these homogeneous spaces to an ODE analysis. For their proof, Abresch and Rosenberg introduced a perturbed Hopf differential, which turned out to be holomorphic for constant mean curvature surfaces in these spaces. The Abresch-Rosenberg theorem was the starting point for the development of the theory of constant mean curvature surfaces in rotationally symmetric homogeneous three-manifolds; see e.g., $[9,11]$ for a survey on the beginnings of this theory.

It is important to observe here that a generic homogeneous three-manifold $M$ has an isometry group of dimension three, and that the techniques used by Abresch and Rosenberg in the rotationally symmetric case do not work to classify constant mean curvature spheres in such an $M$.

In 2013, Daniel and Mira [10] introduced a new method for studying constant mean curvature spheres in the homogeneous manifold $\mathrm{Sol}_{3}$ with its usual Thurston geometry. Using this method, they classified constant mean curvature spheres in $\mathrm{Sol}_{3}$ for values $H$ of the mean curvature greater than $\frac{1}{\sqrt{3}}$, and reduced the general classification problem to the obtention of area estimates for the family of spheres of constant mean curvature greater than any given positive number. These crucial area estimates were subsequently proved by Meeks [23], which completed the classification of constant mean curvature spheres in $\mathrm{Sol}_{3}$ : For any $H>0$ there is a unique constant mean curvature sphere $S_{H}$ in $\mathrm{Sol}_{3}$ with mean curvature $H$; moreover, $S_{H}$ is maximally symmetric, embedded, and has index one [10,23]. 
In [26] we extended the Daniel-Mira theory in [10] to arbitrary simply connected homogeneous three-manifolds $X$ not isometric to $\mathbb{S}^{2}(\kappa) \times \mathbb{R}$. However, the area estimates problem in this general setting cannot be solved following Meeks' approach in $\mathrm{Sol}_{3}$, since the proof by Meeks uses in an essential way special properties of $\mathrm{Sol}_{3}$ that are not shared by a general $X$.

In order to solve the area estimates problem in any homogeneous threemanifold, the authors have developed in previous works an extensive theoretical background for the study of constant mean curvature surfaces in homogeneous three-manifolds, see [24-28]. Specifically, in [28] there is a detailed presentation of the geometry of metric Lie groups, i.e., simply connected homogeneous three-manifolds given by a Lie group endowed with a left invariant metric. In $[26,28]$ we described the basic theory of constant mean curvature surfaces in metric Lie groups. Of special importance for our study here is [26], where we extended the Daniel-Mira theory [10] to arbitrary metric Lie groups $X$, and we proved Theorems 1.1 and 1.2 in the case that $M$ is a homogeneous three-sphere. In [24] we studied the geometry of spheres in metric Lie groups whose left invariant Gauss maps are diffeomorphisms. In [27] we proved that any metric Lie group $X$ diffeomorphic to $\mathbb{R}^{3}$ admits a foliation by leaves of constant mean curvature of value $\mathrm{Ch}(X) / 2$. In [25] we established uniform radius estimates for certain stable minimal surfaces in metric Lie groups. All these works are used in the present manuscript to solve the area estimates problem.

The main results of this paper, Theorems 1.1 and 1.2, can be reduced to demonstrating Theorem 1.5 below; this reduction is explained in Sect. 3. To state Theorem 1.5, we need the notion of entire Killing graph given in the next definition, where $X$ is a simply connected homogeneous three-manifold.

Definition 1.4 Given a nowhere zero Killing field $K$ on $X$, we say that an immersed surface $\Sigma \subset X$ is a Killing graph with respect to $K$ if whenever $\Sigma$ intersects an integral curve of $K$, this intersection is transversal and consists of a single point. If additionally $\Sigma$ intersects every integral curve of $K$, then we say that $\Sigma$ is an entire Killing graph.

By classification, any homogeneous manifold $X$ diffeomorphic to $\mathbb{R}^{3}$ is isometric to a metric Lie group. In this way, any nonzero vector field $K$ on $X$ that is right invariant for such Lie group structure is a nowhere-zero Killing field on $X$. We note that any integral curve of $K$ is a properly embedded curve in $X$ diffeomorphic to $\mathbb{R}$.

Theorem 1.5 Let $X$ be a homogeneous manifold diffeomorphic to $\mathbb{R}^{3}$, and let $S_{n}$ be a sequence of constant mean curvature spheres in $X$ with $\operatorname{Area}\left(S_{n}\right) \rightarrow$ $\infty$. Then, there exist isometries $\Phi_{n}$ of $X$ and compact domains $\Omega_{n} \subset \Phi_{n}\left(S_{n}\right)$ with the property that a subsequence of the $\Omega_{n}$ converges uniformly on compact subsets of $X$ to an entire Killing graph with respect to some nowhere zero 
Killing vector field on $X$, which in fact is right invariant with respect to some Lie group structure on $X$.

One should note that Theorem 1.5 is well-known if the isometry group of $X$ has dimension 6 (equivalently, $X$ has constant curvature) or dimension 4 . For instance, if $X$ has constant curvature $c \leq 0$, constant mean curvature spheres are totally umbilical by Hopf's theorem, and converge in the sense of Theorem 1.5 to planes (if $c=0$ ) or horospheres (if $c<0$ ). If $X$ has an isometry group of dimension four, then constant mean curvature spheres are rotational by the Abresch-Rosenberg theorem, and they converge in the sense of Theorem 1.5 to entire graphs of constant mean curvature in $X$; this follows from classification theorems of rotational CMC surfaces in these spaces by Abresch and Rosenberg [1], Figueroa et al. [12] and Torralbo [42].

The uniqueness statement in Theorem 1.1 relies on the following uniqueness property, whose proof uses in a fundamental way the Poincaré-Hopf index theorem: two spheres $\Sigma_{1}, \Sigma_{2}$ of the same constant mean curvature in a metric Lie group $X$ are congruent provided that the left invariant Gauss map of one of them is a diffeomorphism (see Definition 3.3 for the notion of left invariant Gauss map). This property was proved in [26, Theorem 4.1(2)], and it also follows directly from the general Gálvez-Mira uniqueness theory for immersed spheres in three-manifolds in [13]. A consequence of Theorem 1.5 above will be the existence, in the case that $X$ is diffeomorphic to $\mathbb{R}^{3}$ and for every $H>$ $\mathrm{Ch}(X) / 2$, of a sphere with constant mean curvature $H$ whose left invariant Gauss map is a diffeomorphism. This existence result and the uniqueness property explained above imply the uniqueness statement of Theorem 1.1.

The authors wish to thank the referees for valuable suggestions which led to an improvement of the exposition.

\section{Organization of the paper}

We next explain the organization of the paper and outline the strategy of the proof of the main theorems. In our study in [26] of the space of constant mean curvature spheres of index one in metric Lie groups $X$, we proved that when $X$ is diffeomorphic to $\mathbb{S}^{3}$, Theorems 1.1 and 1.2 hold for $M=X$. In that same paper we proved that if $X$ is a metric Lie group not diffeomorphic to $\mathbb{S}^{3}$ (and hence, diffeomorphic to $\mathbb{R}^{3}$ ), then there exists a constant $h_{0}(X) \geq 0$ such that: (i) for any $H>h_{0}(X)$ there is an index-one sphere $S_{H}$ of constant mean curvature $H$ in $X$; (ii) any sphere of constant mean curvature $H>h_{0}(X)$ is a left translation of $S_{H}$, and (iii) if $H_{n} \searrow h_{0}(X)$, then the areas of the spheres $S_{H_{n}}$ diverge to $\infty$ as $n \rightarrow \infty$; furthermore, the spheres $S_{H_{n}}$ bound isometrically immersed Riemannian three-balls $\widetilde{f}_{n}: B_{n} \rightarrow X$ with the positive mean curvature $H_{n}$; in particular, $S_{H_{n}}$ is Alexandrov-embedded. 
In Sect. 3 we explain how Theorems 1.1 and 1.2 can be deduced from Theorem 1.5 and from the result stated in the last paragraph.

Section 4 is an introductory section; in it we prove some basic properties of constant mean curvature surfaces $f: \Sigma \rightarrow X$ in a metric Lie group $X$ that are invariant, i.e., such that $f(\Sigma)$ is everywhere tangent to a nonzero right invariant Killing vector field $K_{\Sigma}$ on $X$ (equivalently, $f(\Sigma)$ is invariant under the flow of $K_{\Sigma}$ ), and we explain in more detail the geometry of metric Lie groups diffeomorphic to $\mathbb{R}^{3}$. For this analysis, we divide these metric Lie groups into two categories: metric semidirect products and those of the form $(\widetilde{\mathrm{SL}}(2, \mathbb{R}),\langle\rangle$,$) , where \widetilde{\mathrm{SL}}(2, \mathbb{R})$ is the universal cover of the special linear group $\operatorname{SL}(2, \mathbb{R})$ and $\langle$,$\rangle is a left invariant metric; here the metric semidirect$ products $X$ under consideration are the semidirect product of a normal subgroup $\mathbb{R}^{2}$ with $\mathbb{R}$ and we refer to the cosets in $X$ of $\mathbb{R}^{2}$ as being horizontal planes.

In Sect. 5 we prove that if $\left\{S_{n}\right\}_{n}$ is a sequence of index-one spheres with constant mean curvatures of values $H_{n} \searrow h_{0}(X)$ in a metric Lie group $X$ diffeomorphic to $\mathbb{R}^{3}$, then, after taking limits of an appropriately chosen subsequence of left translations of these spheres, there exists an invariant surface $f: \Sigma \leftrightarrow X$ of constant mean curvature $h_{0}(X)$ that is a limit of compact domains of the spheres $S_{n}$. Moreover, $f$ is complete, stable, $\Sigma$ has the topology of a plane or a cylinder, and if $f(\Sigma)$ is not a coset of a two-dimensional subgroup of $X$, then the closure $\overline{\gamma_{f}}$ of its left invariant Gauss map image $\gamma_{f}$ in $\mathbb{S}^{2}$ (see Definition 3.3 for this notion) has the structure of a one-dimensional lamination. Another key property proved in Sect. 5 is that whenever $f(\Sigma)$ is tangent to some coset of a two-dimensional subgroup of $X$, then $f(\Sigma)$ lies in one of the two closed half-spaces bounded by this coset; see Corollary 5.9.

In Sect. 6 we prove in Theorem 6.1 that the invariant limit surface $f(\Sigma)$ can be chosen so that its left invariant Gauss map image $\gamma_{f} \subset \mathbb{S}^{2}$ is a point or a closed embedded regular curve. In the case $\gamma_{f}$ is a point, Theorem 1.5 follows easily from the fact that $f(\Sigma)$ is a coset of a two-dimensional subgroup of $X$. In the case that $\gamma_{f}$ is a closed curve, one can choose $f$ so that one of the following mutually exclusive possibilities occurs:

1. $\Sigma$ is diffeomorphic to an annulus.

2. $\Sigma$ is diffeomorphic to a plane, and $f(\Sigma)$ is an immersed annulus in $X$.

3. $\Sigma$ is diffeomorphic to a plane and there exists an element $a \in X$ such that the left translation by $a$ in $X$ leaves $f(\Sigma)$ invariant, but this left translation does not lie in the 1-parameter subgroup of isometries generated by the nonzero right invariant Killing vector field $K_{\Sigma}$ that is everywhere tangent to $f(\Sigma)$.

In Sect. 7 we prove Theorem 1.5 in the case that $X$ is a metric semidirect product. For that, it suffices to prove that $f(\Sigma)$ is an entire Killing graph for some right invariant Killing vector field $V$ in $X$. This is proved as follows. 
First, we show that if case 3 above holds, then the Killing field $K_{\Sigma}$ which is everywhere tangent to $f(\Sigma)$ is horizontal with respect to the semidirect product structure of $X$, and we prove that $f(\Sigma)$ is an entire graph with respect to any other horizontal Killing field $V$ linearly independent from $K_{\Sigma}$. So, it suffices to rule out cases 1 and 2 above. We prove that case 2 is impossible by constructing in that situation geodesic balls of a certain fixed radius $R^{*}>0$ in the abstract Riemannian three-balls $B_{n}$ that the Alexandrov-embedded indexone spheres $S_{n}$ bound, and whose volumes tend to infinity as $n \rightarrow \infty$. This unbounded volume result eventually provides a contradiction with Bishop's theorem. Finally, we show that case 1 is impossible by constructing an abstract three-dimensional cylinder bounded by $\Sigma$ that submerses isometrically into $X$ with boundary $f(\Sigma)$, and then proving that a certain CMC flux of $\Sigma$ in this abstract cylinder is different from zero. This gives a contradiction with the homological invariance of the CMC flux and the fact that $f(\Sigma)$ is a limit of the (homologically trivial) Alexandrov-embedded constant mean curvature spheres $S_{n}$.

In Sect. 8 we prove Theorem 1.5 in the remaining case where $X$ is isomorphic to $\widetilde{\mathrm{SL}}(2, \mathbb{R})$. The arguments and the basic strategy of the proof in this situation follow closely those from the previous Sect. 7. However, several of these arguments are by necessity different from those in Sect. 7, as many geometric properties of metric semidirect products do not have analogous counterparts in $\operatorname{SL}(2, \mathbb{R})$.

The paper finishes with an Appendix that can be read independently from the rest of the manuscript. In it, we prove a general nonvanishing result for the CMC flux that is used in Sects. 7 and 8 for ruling out case 1 above, as previously explained.

\section{Reduction of Theorems 1.1 and 1.2 to Theorem 1.5}

In order to show that Theorems 1.1 and 1.2 are implied by Theorem 1.5, we will prove the next two assertions in Sects. 3.1 and 3.2, respectively.

Assertion 3.1 Assume that Theorem 1.5 holds. Then Theorems 1.1 and 1.2 hold for the particular case that the homogeneous three-manifold $M$ is simply connected.

Assertion 3.2 Theorems 1.1 and 1.2 hold provided they hold for the particular case that the homogeneous three-manifold $M$ is simply connected.

In what follows, $M$ will denote a homogeneous three-manifold and $X$ will denote its universal Riemannian cover; if $M$ is simply connected, we will identify $M=X$. By an $H$-surface (or $H$-sphere) in $M$ or $X$ we will mean an immersed surface (resp. sphere) of constant mean curvature of value $H \in \mathbb{R}$ immersed in $M$ or $X$. 
The stability operator of an $H$-surface $f: \Sigma q M$ is the Schrödinger operator $L=\Delta+q$, where $\Delta$ stands for the Laplacian with respect to the induced metric in $\Sigma$, and $q$ is the smooth function given by

$$
q=|\sigma|^{2}+\operatorname{Ric}(N)
$$

where $|\sigma|^{2}$ denotes the squared norm of the second fundamental form of $f$, and $N$ is the unit normal vector field to $f$. The immersion $f$ is said to be stable if $-L$ is a nonnegative operator. When $\Sigma$ is closed, the index of $f$ is defined as the number of negative eigenvalues of $-L$, and the nullity of $f$ is the dimension of the kernel of $L$. We say that a function $u \in C^{\infty}(\Sigma)$ is a Jacobi function if $L u=0$. Functions of the type $u=\langle N, F\rangle$ where $F$ is a Killing vector field on the ambient space $M$ are always Jacobi functions on $\Sigma$.

The classification of simply connected homogeneous Riemannian threemanifolds is well-known, and we explain next some of its basic features. For details about what follows, see e.g. Milnor [34], Scott [39] or Sekigawa [40]; see also the notes [28] by two of the authors, which will be used extensively along this paper, and which contain proofs of the results that we detail next.

A simply connected homogeneous three-manifold $X$ has an isometry group $I(X)$ of dimension $n=6,4$ or 3 . If $n=6, M$ is a space of constant curvature $c \in \mathbb{R}$. If $n=4, M$ has rotational symmetry, and is isometric to one of the so-called $\mathbb{E}(\kappa, \tau)$-spaces; see e.g. [8] for a description of their geometries. All these $\mathbb{E}(\kappa, \tau)$ spaces are fibrations over a 2-dimensional manifold $\mathbb{M}^{2}(\kappa)$ of constant curvature $\kappa$, and the constant $\tau$ denotes the bundle curvature of the fibration. At last, in the generic case that $I(X)=3$, the homogeneous manifold $X$ is isometric to a metric Lie group, with a finite isotropy group at any point.

From a topological viewpoint, a simply connected homogeneous threemanifold $X$ is diffeomorphic to $\mathbb{R}^{3}$, to $\mathbb{S}^{3}$ or to $\mathbb{S}^{2} \times \mathbb{R}$. In the $\mathbb{S}^{2} \times \mathbb{R}$ case, $X$ is actually isometric to the product space $\mathbb{S}^{2}(\kappa) \times \mathbb{R}$ for some $\kappa>0$. In all other cases, $X$ is isometric to a metric Lie group. If $X$ is diffeomorphic to $\mathbb{S}^{3}$, the underlying Lie group structure is $\mathrm{SU}(2)$. If $X$ is diffeomorphic to $\mathbb{R}^{3}$, then $X$ is isometric to either a metric semidirect product $\mathbb{R}^{2} \rtimes_{A} \mathbb{R}$ for some $A \in \mathcal{M}_{2}(\mathbb{R})$ equipped with its canonical metric (see Sect. 4.2) or to the universal cover $\widetilde{S L}(2, \mathbb{R})$ of the special linear group with some left invariant metric (see Sect. 4.3). In particular, every simply connected homogeneous Riemannian three-manifold not isometric to $\mathbb{S}^{2}(\kappa) \times \mathbb{R}$ is isometric to a Lie group endowed with a left invariant metric.

\subsection{Proof of Assertion 3.1}

Let $M=X$ denote a simply connected homogeneous three-manifold. When $X$ is isometric to $\mathbb{S}^{2}(\kappa) \times \mathbb{R}$, the statements contained in Theorems 1.1 and 1.2 
follow from the Abresch-Rosenberg theorem that states that constant mean curvature spheres in $\mathbb{S}^{2}(\kappa) \times \mathbb{R}$ are rotational spheres, and from an ODE analysis of the profile curves of these spheres. More specifically, an explicit expression of the rotational $H$-spheres in $\mathbb{S}^{2}(\kappa) \times \mathbb{R}$ can be found in Pedrosa and Ritore [36, Lemma 1.3], or in [1]. From this expression it follows that if $H \neq 0$, then each of these rotational $H$-spheres is embedded, and bounds a unique compact subdomain in $X$ which is a mean convex ball that is invariant under all the isometries of $X$ that fix the mid point in the ball of the revolution axis of the sphere (this point can be defined as the center of symmetry of the sphere). If $H=0$, the corresponding rotational sphere is a slice $\mathbb{S}^{2}(\kappa) \times\left\{t_{0}\right\}$, that is trivially stable, and hence has index zero and nullity one. Moreover, any point of $\mathbb{S}^{2}(\kappa) \times\left\{t_{0}\right\}$ can be defined as the center of symmetry of the sphere in this case, in the sense that any isometry of $\mathbb{S}^{2}(\kappa) \times \mathbb{R}$ that fixes the point must leave invariant $\mathbb{S}^{2}(\kappa) \times\left\{t_{0}\right\}$. That the rotational $H$-spheres in $\mathbb{S}^{2}(\kappa) \times \mathbb{R}$ for $H \neq 0$ have index one and nullity three is shown in Souam [41, proof of Theorem 2.2]. Also, the rotational $H$-spheres for $H>0$ with a fixed center of symmetry $p_{0}$ converge as $H \rightarrow 0$ to a double cover of the slice $\mathbb{S}^{2}(\kappa) \times\left\{t_{0}\right\}$ that contains $p_{0}$. So, from all this information and the Abresch-Rosenberg theorem, it follows that Theorems 1.1 and 1.2 hold when $X$ is isometric to $\mathbb{S}^{2}(\kappa) \times \mathbb{R}$.

When $X$ is diffeomorphic to $\mathbb{S}^{3}$, the statements in Theorems 1.1 and 1.2 were proven in our previous work [26].

So, from now on in this Sect. 3.1, $X$ will denote a homogeneous manifold diffeomorphic to $\mathbb{R}^{3}$. As explained above, $X$ is isometric to a metric Lie group. In the sequel, $X$ will be regarded as a metric Lie group, and $e$ will denote its identity element. Given $x \in X$, we will denote by $l_{x}, r_{x}: X \rightarrow X$ the left and right translations by $x$, respectively given by $l_{x}(y)=x y, r_{x}(y)=y x$ for all $y \in X$. Thus, $l_{x}$ is an isometry of $X$ for every $x \in X$.

Definition 3.3 Given an immersed oriented surface $f: \Sigma \uparrow \rightarrow X$ with unit normal vector field $N: \Sigma \rightarrow T X$ (here $T X$ refers to the tangent bundle of $X$ ), we define the left invariant Gauss map of $f$ to be the map $G: \Sigma \rightarrow \mathbb{S}^{2} \subset T_{e} X$ that assigns to each $p \in \Sigma$, the unit tangent vector $G(p)$ to $X$ at the identity element $e$ given by $\left(d l_{f(p)}\right)_{e}(G(p))=N_{p}$.

The following proposition follows from a rearrangement of ideas taken from [26], but for the sake of clarity, we will include a proof here.

Proposition 3.4 Let $X$ be any metric Lie group $X$ diffeomorphic to $\mathbb{R}^{3}$. Then, there exists a number $h_{0}(X) \geq 0$ such that:

(1) For every $|H|>h_{0}(X)$, there exists an $H$-sphere $S_{H}$ in $X$ with index one and nullity three for its stability operator. Furthermore, $S_{H}$ is Alexandrov embedded, i.e., $S_{H}$ is the boundary of a (unique) mean convex immersed ball $\tilde{f}_{n}: B_{n} \rightarrow X$ in $X$. 
(2) If $\Sigma$ is an $H$-sphere in $X$ for some $|H|>h_{0}(X)$, then $\Sigma$ is a left translation of $S_{H}$.

(3) The left invariant Gauss map $G: S_{H} \rightarrow \mathbb{S}^{2}$ of $S_{H}$ is an orientationpreserving diffeomorphism.

(4) Each $S_{H}$ lies inside a real-analytic family $\left\{\Sigma_{H^{\prime}} \mid H^{\prime} \in(H-\varepsilon, H+\varepsilon)\right\}$ of index-one spheres in $X$ for some $\varepsilon>0$, where $\Sigma_{H}=S_{H}$ and $\Sigma_{H^{\prime}}$ has constant mean curvature of value $H^{\prime}$; note that, by item 2 above, each $\Sigma_{H^{\prime}}$ is a left translation of $S_{H^{\prime}}$. In particular, there exists a unique component $\mathcal{C}$ of the space of index-one spheres with constant mean curvature in $X$ such that $S_{H} \in \mathcal{C}$ for all $H>h_{0}(X)$.

(5) For every $H_{0}>h_{0}(X)$ there exists some positive constant $C=C\left(X, H_{0}\right)$ such that if $|H| \in\left(h_{0}(X), H_{0}\right]$, then the norm of the second fundamental form of $S_{H}$ is at most $C$.

(6) For every $H_{0}>h_{0}(X)$ there exists some positive constant $D=D\left(X, H_{0}\right)$ such that if $|H|>H_{0}$, then the area of $S_{H}$ is at most $D$.

(7) If $S_{n}$ is a sequence of constant mean curvature spheres in $X$ whose mean curvatures $H_{n}>h_{0}(X)$ satisfy $H_{n} \rightarrow h_{0}(X)$, then $\operatorname{Area}\left(S_{n}\right) \rightarrow \infty$.

Proof After a change of orientation if necessary, it clearly suffices to prove all statements for the case where $H \geq 0$. By [26, item 3 of Theorem 4.1], there exists a unique component $\mathcal{C}$ of the space of index-one spheres with constant mean curvature in $X$ such that the values of the mean curvatures of the spheres in $\mathcal{C}$ are not bounded from above. As $X$ is diffeomorphic to $\mathbb{R}^{3}$, then [26, item 6 of Theorem 4.1] ensures that the map $\mathcal{H}: \mathcal{C} \rightarrow \mathbb{R}$ that assigns to each sphere in $\mathcal{C}$ its mean curvature is not surjective. By item 5 in the same theorem, there exists $h_{0}(X) \geq 0$ such that for every $H>h_{0}(X)$, there is an index-one sphere $S_{H} \in \mathcal{C}$, and the following property holds:

(7) $)^{\prime}$ The areas of any sequence $\left\{S_{H_{n}}\right\}_{n} \subset \mathcal{C}$ with $H \rightarrow h_{0}(X)$ satisfy $\lim _{n \rightarrow \infty} \operatorname{Area}\left(S_{H_{n}}\right)=\infty$.

Since the sphere $S_{H}$ has index one, then Cheng [5, Theorem 3.4] gives that the nullity of $S_{H}$ is three. Alexandrov embeddedness of $S_{H}$ follows from [26, Corollary 4.4]. Now item 1 of Proposition 3.4 is proved.

Once this item 1 has been proved, items 2, 3, 4, 5 of Proposition 3.4 are proved in items 1, 2, 3, 4 of [26, Theorem 4.1]. Item 6 of Proposition 3.4 is a direct consequence of the existence, uniqueness and analyticity properties of the spheres $S_{H}$ stated in items 1,2, 4, and of the fact also proved in [26] that, for $H$ large enough, the spheres $S_{H}$ bound small isoperimetric regions in $X$. Finally, item 7 of Proposition 3.4 follows from property (7) ${ }^{\prime}$ above and from the uniqueness given by the already proven item 2 of Proposition 3.4. Now the proof is complete.

A key notion in what follows is the critical mean curvature of $X$. 
Definition 3.5 Let $X$ be a homogeneous manifold diffeomorphic to $\mathbb{R}^{3}$, let $\mathcal{A}(X)$ be the collection of all closed, immersed surfaces in $X$, and given a surface $\Sigma \in \mathcal{A}(X)$, let $\left|H_{\Sigma}\right|: \Sigma \rightarrow[0, \infty)$ stand for the absolute mean curvature function of $\Sigma$. The critical mean curvature of $X$ is defined as

$$
H(X)=\inf \left\{\max _{\Sigma}\left|H_{\Sigma}\right|: \Sigma \in \mathcal{A}(X)\right\} .
$$

Item 2 of Theorem 1.4 in [27] gives that $\mathrm{Ch}(X)=2 H(X)$. By definition of $H(X)$, every compact $H$-surface in $X$ satisfies $|H| \geq H(X)$. Observe that Proposition 3.4 gives that for every $H>h_{0}(X)$ there is an $H$-sphere $S_{H}$ in $X$. Thus,

$$
h_{0}(X) \geq H(X)=\mathrm{Ch}(X) / 2 .
$$

In order to prove Assertion 3.1, in the remainder of this section we will assume that Theorem 1.5 holds. Actually, we will just assume that Theorem 1.5 holds for any sequence $S_{n}$ of spheres in $X$ satisfying the conditions of item 7 of Proposition 3.4.

We will prove next that $h_{0}(X)=H(X)$. If $S_{n}$ are constant mean curvature spheres satisfying item 7 of Proposition 3.4, then by Theorem 1.5 we obtain the existence of a surface $\Sigma_{0}$ in $X$ of constant mean curvature $h_{0}(X)$ that is an entire Killing graph with respect to some nonzero right invariant Killing field $K$. Let $\Gamma=\{\Gamma(t) \mid t \in \mathbb{R}\}$ be the 1-parameter subgroup of $X$ given by $\Gamma(0)=e, \Gamma^{\prime}(0)=K(e)$. As left translations are isometries of $X$, it follows that $\left\{l_{a}\left(\Sigma_{0}\right) \mid a \in \Gamma\right\}$ defines a foliation of $X$ by congruent surfaces of constant mean curvature $h_{0}(X)$, and this foliation is topologically a product foliation. A standard application of the mean curvature comparison principle shows then that there are no closed surfaces $\Sigma$ in $X$ with $\max _{\Sigma}|H|<h_{0}(X)$ (otherwise, we left translate $\Sigma_{0}$ until $\Sigma$ is contained in the region of $X$ on the mean convex side of $\Sigma_{0}$, and then start left translating $\Sigma_{0}$ towards its mean convex side until it reaches a first contact point with $\Sigma$; this provides a contradiction with the mean curvature comparison principle). Therefore, we conclude that $h_{0}(X)=H(X)$ and that, by item 1 of Proposition 3.4, the values $H \in \mathbb{R}$ for which there exists a sphere of constant mean curvature $H$ in $X$ are exactly those with $|H|>H(X)$. This fact together with item 2 of Proposition 3.4 proves item 2 of Theorem 1.1, and thus it completes the proof of Theorem 1.1 in the case $X$ is diffeomorphic to $\mathbb{R}^{3}$ (assuming that Theorem 1.5 holds).

Regarding the proof of Theorem 1.2 when $X$ is diffeomorphic to $\mathbb{R}^{3}$, similar arguments as the one in the preceding paragraph prove item 2 of Theorem 1.2. To conclude the proof of Theorem 1.2 (and hence of Assertion 3.1) we need to show that all spheres $S_{H}$ in $X$ are maximally symmetric, something that we will prove next. We note that the 'Moreover' part in the statement of Theorem 1.2 
follows directly from the existence of a center of symmetry of each sphere $S_{H}$, and the analyticity properties in item 4 of Proposition 3.4.

In the case that the isometry group $I(X)$ of $X$ is of dimension 6 , all constant mean curvature spheres in $X$ are totally umbilical; in particular they are maximally symmetric, and the definition of the center of symmetry of the sphere is clear.

When $I(X)$ has dimension 4, it follows from the Abresch-Rosenberg theorem that constant mean curvature spheres in $X$ are rotational, and thus they can be explicitly classified. In particular, if $X$ is diffeomorphic to $\mathbb{R}^{3}$, these spheres are embedded and intersect their rotation axis orthogonally at exactly two points. In this way, we may define their center of symmetry as the midpoint of these two intersection points along the rotation axis. It can be checked that, with this definition, any constant mean curvature sphere in such space $X$ is invariant with respect to any ambient isometry that fixes its center of symmetry, and in particular they are maximally symmetric.

So, in the remainder of this section we deal with the case that $X$ is a metric Lie group diffeomorphic to $\mathbb{R}^{3}$ whose isometry group $I(X)$ has dimension 3 . Let $\operatorname{Stab}_{e}(X)$ denote the group of isometries $\psi$ of $X$ with $\psi(e)=e$, and let $\mathrm{Stab}_{e}^{+}(X)$ denote the index-two subgroup of orientation-preserving isometries in $\operatorname{Stab}_{e}(X)$. The next proposition gives some basic properties of the elements of $\operatorname{Stab}_{e}(X)$ that will be needed for proving the maximal symmetry of $H$ spheres in $X$. Its proof follows from the analysis of metric Lie groups in [28].

Proposition 3.6 Assume that $I(X)$ has dimension three. Then, there exists a 1-parameter subgroup $\Gamma$ of $X$ such that:

(1) There exists an isometry $\phi \in \operatorname{Stab}_{e}^{+}(X)$ of order two in $X$ that is a group automorphism of $X$, and whose fixed point set is $\Gamma$, i.e., $\Gamma=\{x \in X$ : $\phi(x)=x\}$.

(2) Every $\psi \in \operatorname{Stab}_{e}(X)$ leaves $\Gamma$ invariant, in the sense that $\psi(\Gamma)=\Gamma$.

Moreover, if there exists another 1-parameter subgroup $\widehat{\Gamma} \neq \Gamma$ of $X$ that satisfies the previous two properties, then $\phi(\widehat{\Gamma})=\widehat{\Gamma}$, where $\phi$ is given with respect to $\Gamma$ by item 1 above.

Proof First suppose that the underlying Lie group structure $Y$ of $X$ is not unimodular ${ }^{1}$. Hence $Y$ is isomorphic to some semidirect product $\mathbb{R}^{2} \rtimes_{A} \mathbb{R}$ for some matrix $A \in \mathcal{M}_{2}(\mathbb{R})$ with $\operatorname{trace}(A) \neq 0$ and $\operatorname{det}(A) \neq 0$ since the dimension of $I(X)=3$; see Sect. 4.2 for this notion of nonunimodular metric Lie group. Then [28, item 4 of Proposition 2.21] gives that $\operatorname{Stab}_{e}^{+}(X)=\left\{1_{X}, \phi\right\}$ where $\phi(x, y, z)=(-x,-y, z)$. Therefore, item 1 of Proposition 3.6 holds with the choice $\Gamma=\{(0,0, z) \mid z \in \mathbb{R}\}$ and the above $\phi$. Regarding item 2, we

\footnotetext{
1 A Lie group with Lie algebra $\mathfrak{g}$ is called unimodular if for all $V \in \mathfrak{g}$, the Lie algebra endo-

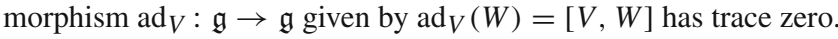


divide the argument into two cases in this nonunimodular case for $Y$. Given $b \in \mathbb{R}-\{0\}$, consider the matrix

$$
A(b)=\left(\begin{array}{ll}
1 & 0 \\
0 & b
\end{array}\right)
$$

(A1) If $X$ is not isomorphic and homothetic to any $\mathbb{R}^{2} \rtimes_{A(b)} \mathbb{R}$ with $A$ given by (3.2) with $b \neq-1$, then [28, item 3 of Proposition 2.24] gives that $\operatorname{Stab}_{e}(X)=\operatorname{Stab}_{e}^{+}(X)$.

(A2) If $X$ is isomorphic and homothetic to $\mathbb{R}^{2} \rtimes_{A(b)} \mathbb{R}$ for some $b \neq-1,0$, then [28, item (3a) of Proposition 2.24] implies that then $\operatorname{Stab}_{e}(X)-$ $\operatorname{Stab}_{e}^{+}(X)=\left\{\psi_{1}, \psi_{2}\right\}$ where $\psi_{1}(x, y, z)=(-x, y, z)$ and $\psi_{2}(x, y, z)=$ $(x,-y, z)$. In the case that $b=0$, then $\operatorname{det}(A(b))=0$, which is not the case presently under consideration as the isometry group of $X$ for $b=0$ has dimension four.

In both cases (A1) and (A2), item 2 of Proposition 3.6 holds with $\Gamma$ being the $z$-axis. The moreover part of Proposition 3.6 also holds, because item 1 only holds for $\Gamma=\{(0,0, z) \mid z \in \mathbb{R}\}$.

Assume next that $Y$ is unimodular. Since $\operatorname{dim}(I(X))=3$ and $Y$ is unimodular, then there exists a frame of left invariant vector fields $E_{1}, E_{2}, E_{3}$ on $Y$ that are eigenfields of the Ricci tensor of $X$, independently of the left invariant metric on $X$, see [28, Section 2.6]. By the proof of Proposition 2.21 of [28], for each $i=1,2,3$ there exists an orientation-preserving automorphism $\phi_{i}: X \rightarrow X$ whose differential satisfies $\left(\phi_{i}\right)_{*}\left(E_{i}\right)=E_{i}$ and $\left(\phi_{i}\right)_{*}\left(E_{j}\right)=-E_{j}$ whenever $j \neq i$. Furthermore, $\phi$ is an isometry of every left invariant metric on $Y$ (in particular, of the metric of $X$ ). Also, item 1 of Proposition 2.24 in [28] gives that $\operatorname{Stab}_{e}^{+}(X)$ is the dihedral group $\left\{1_{X}, \phi_{1}, \phi_{2}, \phi_{3}\right\}$. Therefore, item 1 of Proposition 3.6 holds with any of the choices $\phi=\phi_{i}$ and $\Gamma=\Gamma_{i}$, for $i=1,2,3$, where $\Gamma_{i}$ is the 1-parameter subgroup of $X$ generated by $\left(E_{i}\right)_{e}$. Regarding item 2, we again divide the argument into two cases in this unimodular case for $Y$.

(A1)' If $X$ is not isomorphic and homothetic to $\mathbb{R}^{2} \rtimes_{A(-1)} \mathbb{R}$ (recall that $\mathbb{R}^{2} \rtimes_{A(-1)} \mathbb{R}$ is $\mathrm{Sol}_{3}$ with its standard metric), then [28, item 3 of Proposition 2.24] ensures that $\operatorname{Stab}_{e}(X)=\operatorname{Stab}_{e}^{+}(X)$. Thus item 2 of Proposition 3.6 holds for every choice of the form $\Gamma=\Gamma_{i}$ and $\phi=\phi_{i}$ with $i=1,2,3$. Once here, and since it is immediate that $\phi_{i}\left(\Gamma_{j}\right)=\Gamma_{j}$ for any $i, j \in\{1,2,3\}$, we conclude that the moreover part of Proposition 3.6 holds in this case.

(A2) If $X$ is isomorphic and homothetic to $\mathbb{R}^{2} \rtimes_{A(-1)} \mathbb{R}$, then [28, item $3 \mathrm{~b}$ of Proposition 2.24] gives that $\operatorname{Stab}_{e}(X)-\operatorname{Stab}_{e}^{+}(X)=\left\{\psi_{1}, \psi_{2}, \psi_{3}, \psi_{4}\right\}$, 
where

$$
\begin{array}{ll}
\psi_{1}(x, y, z)=(-x, y, z), & \psi_{2}(x, y, z)=(x,-y, z), \\
\psi_{3}(x, y, z)=(y,-x,-z), & \psi_{4}(x, y, z)=(-y, x,-z) .
\end{array}
$$

Therefore, item 2 of Proposition 3.6 only holds for the choice $\Gamma=$ $\{(0,0, z) \mid z \in \mathbb{R}\}$ and $\phi(x, y, z)=(-x,-y, z)$ (in particular, the moreover part of Proposition 3.6 also holds).

Now the proof is complete.

We are now ready to prove the existence of the center of symmetry of any $H$-sphere in $X$, in the remaining case that $I(X)$ has dimension three. Let $f_{H}: S_{H} \leftrightarrow X$ denote an $H$-sphere, with left invariant Gauss map $G: S_{H} \rightarrow \mathbb{S}^{2}$. By item 3 of Proposition 3.4 and $h_{0}(X)=H(X)$, we know $G$ is an orientation preserving diffeomorphism. Without loss of generality we can assume that $f_{H}\left(q_{H}\right)=e$, where $q_{H} \in S_{H}$ is the unique point such that $G\left(q_{H}\right)=v:=\Gamma^{\prime}(0)$ and $\Gamma: \mathbb{R} \rightarrow X$ is an arc length parameterization of the 1-parameter subgroup of $X$ that appears in Proposition 3.6. Let $\phi \in \operatorname{Stab}_{e}^{+}(X)$ be an isometry associated to $\Gamma$ in the conditions of item 1 of Proposition 3.6. Clearly $d \phi_{e}(v)=v$, hence $f_{H}\left(S_{H}\right)$ and $\left(\phi \circ f_{H}\right)\left(S_{H}\right)$ are two $H$-spheres in $X$ passing through $e$ with the same left invariant Gauss map image at $e$. By the uniqueness of $H$-spheres up to left translations in item 2 of Proposition 3.4, we conclude that $f_{H}\left(S_{H}\right)=\left(\phi \circ f_{H}\right)\left(S_{H}\right)$. Let $q_{H}^{*}$ be the unique point in $S_{H}$ such that $G\left(q_{H}^{*}\right)=-v$. Since $d \phi_{e}(-v)=-v$, we see that the Gauss map image of $\phi \circ f_{H}: S_{H} \hookrightarrow X$ at $q_{H}^{*}$ is also $-v$. Since $f_{H}\left(S_{H}\right)=\left(\phi \circ f_{H}\right)\left(S_{H}\right)$, this implies (by uniqueness of the point $q_{H}^{*}$ ) that $f_{H}\left(q_{H}^{*}\right)$ is a fixed point of $\phi$, which by item 1 of Proposition 3.6 shows that $f_{H}\left(q_{H}^{*}\right) \in \Gamma$.

Let $x_{H} \in \Gamma$ be the midpoint of the connected arc of $\Gamma$ that connects $e$ with $f_{H}\left(q_{H}^{*}\right)$, and define $\widehat{f}_{H}:=l_{x_{H}^{-1}} \circ f_{H}: S_{H} \uparrow X$. As the family $\left\{f_{H}: S_{H} \uparrow X: H>H(X)\right\}$ is real analytic with respect to $H$ (by items 2 and 4 of Proposition 3.4), then the family $\left\{\widehat{f}_{H}: S_{H} \rightarrow X \mid H>H(X)\right\}$ is also real analytic in terms of $H$. Also, note that the points $p_{H}:=\widehat{f}_{H}\left(q_{H}\right)$ and $p_{H}^{*}:=\widehat{f}_{H}\left(q_{H}^{*}\right)$ both lie in $\Gamma$, and are equidistant from $e$ along $\Gamma$. This property and item 2 of Proposition 3.6 imply that every $\psi \in \operatorname{Stab}_{e}(X)$ satisfies $\psi\left(\left\{p_{H}, p_{H}^{*}\right\}\right)=\left\{p_{H}, p_{H}^{*}\right\}$, and hence either $\psi\left(p_{H}\right)=p_{H}$ and $\psi\left(p_{H}^{*}\right)=p_{H}^{*}$, or alternatively $\psi\left(p_{H}\right)=p_{H}^{*}$ and $\psi\left(p_{H}^{*}\right)=p_{H}$.

Take $\psi \in \operatorname{Stab}_{e}(X)$. We claim that $\psi$ leaves $\widehat{f}_{H}\left(S_{H}\right)$ invariant. To see this, we will distinguish four cases.

(B1) Suppose that $\psi$ preserves orientation on $X$ and satisfies $\psi\left(p_{H}\right)=p_{H}$, $\psi\left(p_{H}^{*}\right)=p_{H}^{*}$. The first condition implies that $\left(\psi \circ \widehat{f}_{H}\right)\left(S_{H}\right)$ is an $H$-sphere in $X$. So, by items 2 and 3 of Proposition 3.4 we have $l_{a}\left(\widehat{f}_{H}\left(S_{H}\right)\right)=\left(\psi \circ \widehat{f}_{H}\right)\left(S_{H}\right)$ for some $a \in X$, and that $l_{a}\left(p_{H}\right)$ corresponds to the unique point of $\left(\psi \circ \widehat{f}_{H}\right)\left(S_{H}\right)$ where its Gauss map takes 
the value $v$. But now, from the second condition we obtain that $\psi$ restricts to $\Gamma$ as the identity map, which implies that the value of the left invariant Gauss map image of $\left(\psi \circ \widehat{f}_{H}\right)\left(S_{H}\right)$ at $p_{H}$ is $v$. Thus, $l_{a}\left(p_{H}\right)=p_{H}$, hence $a=e$ and $\widehat{f}_{H}\left(S_{H}\right)=\left(\psi \circ \widehat{f}_{H}\right)\left(S_{H}\right)$, that is, $\psi$ leaves $\widehat{f}_{H}\left(S_{H}\right)$ invariant.

(B2) Suppose that $\psi$ reverses orientation on $X$ and satisfies $\psi\left(p_{H}\right)=p_{H}^{*}$, $\psi\left(p_{H}^{*}\right)=p_{H}$. The first condition implies that after changing the orientation of $\left(\psi \circ \widehat{f}_{H}\right)\left(S_{H}\right)$, this last surface is an $H$-sphere in $X$. Since by the second condition $\psi$ restricts to $\Gamma$ as minus the identity map, then the left invariant Gauss map image of $\left(\psi \circ \widehat{f}_{H}\right)\left(S_{H}\right)$ (with the reversed orientation) at $p_{H}$ is $v$. Now we deduce as in case (B1) that $\psi$ leaves $\widehat{f}_{H}\left(S_{H}\right)$ invariant.

(B3) Suppose that $\psi$ reverses orientation on $X$ and satisfies $\psi\left(p_{H}\right)=p_{H}$, $\psi\left(p_{H}^{*}\right)=p_{H}^{*}$. As in case (B2), we change the orientation on $(\psi \circ$ $\left.\widehat{f}_{H}\right)\left(S_{H}\right)$ so it becomes an $H$-sphere in $X$. Item 2 of Proposition 3.4 then gives that there exists $a \in X$ such that $\left(\psi \circ \widehat{f}_{H}\right)\left(S_{H}\right)=l_{a}\left(\widehat{f}_{H}\left(S_{H}\right)\right)$. As $p_{H}^{*} \in \widehat{f}_{H}\left(S_{H}\right) \cap \Gamma$ and $\psi$ fixes $\Gamma$ pointwise, then $p_{H}^{*} \in\left(\psi \circ \widehat{f}_{H}\right)\left(S_{H}\right)$. Moreover, the value of the left invariant Gauss map image of $\left(\psi \circ \widehat{f}_{H}\right)\left(S_{H}\right)$ (with the reversed orientation) at $p_{H}^{*}$ is $v$. Since the only point of $l_{a}\left(\widehat{f}_{H}\left(S_{H}\right)\right)$ where its left invariant Gauss map image (with the original orientation) takes the value $v$ is $l_{a}\left(p_{H}\right)$, we deduce that $l_{a}\left(p_{H}\right)=p_{H}^{*}$. Similarly, $p_{H}=l_{a}\left(p_{H}^{*}\right)$. This is only possible if $a=a^{-1}$, which, in our setting that $X$ is diffeomorphic to $\mathbb{R}^{3}$, implies that $a=e$ (note that this does not happen when $X$ is diffeomorphic to the three-sphere, because then $X$ is isomorphic to $\mathrm{SU}(2)$, which has one element of order 2). The claim then holds in this case.

(B4) Finally, assume that $\psi$ preserves the orientation on $X$ and satisfies $\psi\left(p_{H}\right)=p_{H}^{*}, \psi\left(p_{H}^{*}\right)=p_{H}$. As in case $(\mathrm{B} 1),\left(\psi \circ \widehat{f}_{H}\right)\left(S_{H}\right)$ is an $H$-sphere in $X$ and so there exists $a \in X$ such that $\left(\psi \circ \widehat{f}_{H}\right)\left(S_{H}\right)=$ $l_{a}\left(\widehat{f}_{H}\left(S_{H}\right)\right)$. As $p_{H} \in \widehat{f}_{H}\left(S_{H}\right)$, then $p_{H}^{*}=\psi\left(p_{H}\right) \in\left(\psi \circ \widehat{f}_{H}\right)\left(S_{H}\right)$. Since $\psi$ restricts to $\Gamma$ as minus the identity map, then the value of the left invariant Gauss map image of $\left(\psi \circ \widehat{f}_{H}\right)\left(S_{H}\right)$ at $p_{H}^{*}$ is $v$. Since the only point of $l_{a}\left(\widehat{f}_{H}\left(S_{H}\right)\right)$ where its left invariant Gauss map image takes the value $v$ is $l_{a}\left(p_{H}\right)$, we deduce that $l_{a}\left(p_{H}\right)=p_{H}^{*}$. Now we finish as in case (B3).

To sum up, we have proved that any $\psi \in \operatorname{Stab}_{e}(X)$ leaves $\widehat{f}_{H}\left(S_{H}\right)$ invariant; this proves that the $H$-sphere $\widehat{f}_{H}\left(S_{H}\right)$ is maximally symmetric with respect to the identity element $e$, in the sense of item 1 of Theorem 1.2. So, we may define $e$ to be the center of symmetry of $\widehat{f}_{H}\left(S_{H}\right)$. We next show that the above definition of center of symmetry does not depend on the choice of the 1-parameter subgroup $\Gamma$ of $X$ that appears in Proposition 3.6. Suppose $\widehat{\Gamma}$ is another 1-parameter subgroup of $X$ satisfying the conclusions of 
Proposition 3.6. As we explained in the paragraph just after Proposition 3.6, $\widehat{\Gamma}$ determines two points $\widehat{q}, \widehat{q}^{*} \in S_{H}$ with $\widehat{f}_{H}(\widehat{q}), \widehat{f}_{H}\left(\widehat{q}^{*}\right) \in \widehat{\Gamma}$, such that $G(\widehat{q})=\widehat{\Gamma}^{\prime}(0), G\left(\widehat{q}^{*}\right)=-\widehat{\Gamma}^{\prime}(0)$, where $G$ is the left invariant Gauss map of $\widehat{f}_{H}$. To show that the above definition of center of symmetry of $\widehat{f}_{H}\left(S_{H}\right)$ does not depend on $\Gamma$, we must prove that the mid point of the arc of $\widehat{\Gamma}$ between $\widehat{f_{H}}(\widehat{q})$ an $\widehat{f}_{H}\left(\widehat{q}^{*}\right)$ is $e$. To do this, let $\phi \in \operatorname{Stab}_{e}^{+}(X)$ be an order-two isometry that satisfies item 1 of Proposition 3.6 with respect to $\Gamma$. By Proposition 3.6, $\phi(\widehat{\Gamma})=\widehat{\Gamma}$. As $\phi$ leaves $\widehat{f}_{H}\left(S_{H}\right)$ invariant and $G$ is a diffeomorphism, then $\phi\left(\left\{\widehat{f}_{H}(\widehat{q}), \widehat{f}_{H}\left(\widehat{q}^{*}\right)\right\}\right)=\left\{\widehat{f_{H}}(\widehat{q}), \widehat{f}_{H}\left(\widehat{q}^{*}\right)\right\}$. Since the set of fixed points of $\phi$ is $\Gamma$ and $\widehat{f}_{H}(\widehat{q}), \widehat{f}_{H}\left(\widehat{q}^{*}\right) \notin \Gamma$ (because two different 1-parameter subgroups in $X$ only intersect at $e$, here we are using again that $X$ is diffeomorphic to $\mathbb{R}^{3}$ ), then $\phi\left(\widehat{f}_{H}(\widehat{q})\right)=\widehat{f}_{H}\left(\widehat{q}^{*}\right)$. As $\phi$ is an isometry with $\phi(e)=e$ and $\phi(\widehat{\Gamma})=\widehat{\Gamma}$, we deduce that the lengths of the arcs of $\widehat{\Gamma}$ that join $e$ to $\widehat{f}_{H}(\widehat{q})$, and $e$ to $\widehat{f}_{H}\left(\widehat{q}^{*}\right)$, coincide. This implies that the mid point of the arc of $\widehat{\Gamma}$ between $\left.\widehat{f}_{H}(\widehat{q})\right)$ an $\widehat{f}_{H}\left(\widehat{q}^{*}\right)$ is $e$, as desired. As every $H$-sphere in $X$ is a left translation of $\widehat{f}_{H}\left(S_{H}\right)$, we conclude that all $H$-spheres in $X$ are maximally symmetric with respect to some point $p \in X$, which is obtained by the corresponding left translation of the identity element $e$, and thus can be defined as the center of symmetry of the sphere. This completes the proof of Theorem 1.2 (assuming that Theorem 1.5 holds) for the case that $X$ is diffeomorphic to $\mathbb{R}^{3}$. Thus, the proof of Assertion 3.1 is complete.

The next remark gives a direct definition of the center of symmetry of any $H$-sphere in $X$ :

Remark 3.7 (Definition of the center of symmetry). Let $f: S_{H} \rightarrow X$ be an $H$-sphere in a homogeneous manifold $X$ diffeomorphic to $\mathbb{R}^{3}$ with an isometry group of dimension three. Let $q, q^{*} \in S_{H}$ be given by $G(q)=v, G\left(q^{*}\right)=$ $-v$, where $G: S_{H} \rightarrow \mathbb{S}^{2}$ denotes the left invariant Gauss map of $S_{H}$ and $v:=\Gamma^{\prime}(0)$, with $\Gamma(t)$ a 1-parameter subgroup satisfying the conditions in Proposition 3.6. Then, by our previous discussion, the left coset $\alpha:=l_{f(q)}(\Gamma)$ passes through both $f(q)$ and $f\left(q^{*}\right)$, and we define the center of symmetry of $S_{H}$ as the midpoint $p \in \alpha$ of the subarc of $\alpha$ that joins $f(q)$ with $f\left(q^{*}\right)$.

It is worth mentioning that this definition gives a nonambiguous definition of the center of symmetry of an $H$-sphere; however, when $X$ has an isometry group of dimension three and is nonunimodular, there are many points $p \in X$ besides this center of symmetry such that the $H$-sphere is invariant under all isometries of $X$ that fix $p$. Nevertheless, in order to make sense of the analyticity properties of the family of constant mean curvature spheres with a fixed center of symmetry (see Theorem 1.2), we need to make the definition of center of symmetry nonambiguous. 


\subsection{Proof of Assertion 3.2}

Let $M$ be a homogeneous three-manifold with universal covering space $\Pi: X \rightarrow M$. To prove Assertion 3.2, we will assume in this Sect. 3.2 that Theorems 1.1 and 1.2 hold for $X$.

We first prove Theorem 1.1 in $M$. Since every constant mean curvature sphere $f: S \leftrightarrow M$ is the projection via $\Pi$ of some lift $\widetilde{f}: S \rightarrow X$ of $f$ with the same mean curvature, then clearly the first and second items in Theorem 1.1 hold in $M$, since they are true by hypothesis in $X$. In order to prove the uniqueness statement in Theorem 1.1, let $f_{1}: S_{1} \rightarrow M, f_{2}: S_{2} \rightarrow M$ be two spheres with the same constant mean curvature in $M$. For $i=1,2$, choose $\widetilde{f}_{i}$ respective lifts of $f_{i}$, let $\widetilde{p}_{i}$ be the centers of symmetry of $\widetilde{f}_{i}$, denote $p_{i}=\Pi\left(\widetilde{p}_{i}\right)$, and let $I: M \rightarrow M$ be an isometry with $I\left(p_{1}\right)=p_{2}$. Let $\widetilde{I}: X \rightarrow X$ be the lift of $I$ that takes $\widetilde{p}_{1}$ to $\widetilde{p}_{2}$. It follows that the $H$-spheres $\widetilde{I} \circ \widetilde{f}_{1}$ and $\widetilde{f}_{2}$ have the same center of symmetry in $X$ and so these immersions have the same images. In particular, it follows that $I \circ f_{1}$ and $f_{2}$ also have the same images, which completes the proof of Theorem 1.1 in $M$.

We next prove Theorem 1.2 in $M$. Suppose $f: S \rightarrow M$ is an oriented $H$ sphere, and let $\widetilde{f}: S \rightarrow X$ denote some lift of $f$. Since the stability operators of $f$ and $\widetilde{f}$ are the same, the index and nullity of $f$ and $\widetilde{f}$ agree. Also, note that if the immersion $\widetilde{f}$ extends to an isometric immersion $F: B \rightarrow X$ of a mean convex Riemannian three-ball $B$ into $X$, then $\Pi \circ F$ is an isometric immersion of $B$ into $M$ that extends $f$. These two trivial observations prove that item 2 of Theorem 1.2 holds in $M$.

If $M$ is covered by $\mathbb{S}^{2}(\kappa) \times \mathbb{R}$ and $f(S)$ is totally geodesic, then any point of the image sphere satisfies the property of being a center of symmetry of $f$. This observation follows by the classification of homogeneous three-manifolds covered by $\mathbb{S}^{2}(\kappa) \times \mathbb{R}$ as being those spaces isometric to $\mathbb{S}^{2}(\kappa) \times \mathbb{R}, \mathbb{S}^{2}(\kappa) \times$ $\mathbb{S}^{1}(R), \mathbb{P}^{2}(\kappa) \times \mathbb{R}$ or $\mathbb{P}^{2}(\kappa) \times \mathbb{S}^{1}(R)$, where $\mathbb{S}^{1}(R)$ is a circle of circumference $R$ for some $R>0$ and $\mathbb{P}^{2}(\kappa)$ is the projective plane of constant Gaussian curvature $\kappa>0$.

Suppose now that $M$ is not covered by $\mathbb{S}^{2}(\kappa) \times \mathbb{R}$ with $f(S)$ being totally geodesic. In order to complete the proof of item 1 of Theorem 1.2, we next define the center of symmetry of $f$ in $M$ as $p:=\Pi(\widetilde{p})$, where $\widetilde{p}$ is the center of symmetry of a lift $\widetilde{f}$ in $X$. Since the center of symmetry of the lift $\widetilde{f}$ is uniquely defined by $\widetilde{f}$ and any other lift is equal to the composition of $\widetilde{f}$ with an isometry $\sigma: X \rightarrow X$ that is a covering transformation, then by uniqueness of the center of symmetry in $X, \sigma$ maps the center of symmetry of $\tilde{f}$ to the center of symmetry of the composed oriented immersion $\sigma \circ \widetilde{f}$; hence $p$ is independent of the choice of the lift of $f$ to $X$. We now show that $f(S)$ is invariant under all isometries of $M$ that fix $p$. Let $I: M \rightarrow M$ be any such isometry, and let $\widetilde{I}: X \rightarrow X$ be the lift of $I$ such that $\widetilde{I}(\widetilde{p})=\widetilde{p}$. As $\widetilde{p}$ is 
a center of symmetry of $\tilde{f}$, then, $\widetilde{I}$ induces an isometry of $\tilde{f}: S \leftrightarrow X$. It follows that $I$ induces an isometry of the $H$-sphere $f$, which proves item 1 of Theorem 1.2 in $M$. Once here, the homeomorphism and analyticity properties in the last statement of Theorem 1.2 follow trivially from the validity of the corresponding statement in $X$. This completes the proof of Theorem 1.2 in $M$, and thus also the proof of Assertion 3.2.

\subsection{Constant mean curvature spheres in complete locally homogeneous three-manifolds}

As a consequence of Theorems 1.1 and 1.2 and of the discussion in Sects. 3.1 and 3.2 we can provide a description of the space of constant mean curvature spheres in complete, locally homogeneous three-manifolds. Specifically, let $Y$ be a complete, connected, locally homogeneous Riemannian three-manifold. Then, the universal Riemannian covering space $\Pi: X \rightarrow Y$ is a simply connected homogeneous three-manifold. Let $\mathrm{Ch}(X)$ be the Cheeger constant of $X$. Remark 3.7, the proof of Proposition 3.6 and the results on the existence and uniqueness of the center of symmetry for any $H$-sphere in $X$ imply that associated to any $H$-sphere $S_{H}$ in $Y$ with $H>0$, there is a unique point $p\left(S_{H}\right) \in Y$ that is the image by $\Pi$ of the center of symmetry of any lift of $S_{H}$ to $X$; that $p\left(S_{H}\right)$ does not depend on the lift of $S_{H}$ can be proved following the arguments used in Sect. 3.2. The following properties of spheres with nonzero constant mean curvature in $Y$ are then easy to check, and provide a parameterization of the space of such constant mean curvature spheres.

1. For any $H>\mathrm{Ch}(X) / 2$ and any $p_{0} \in Y$ there exists a unique $H$-sphere $S_{H}$ in $Y$ with $p\left(S_{H}\right)=p_{0}$.

2. If $\mathrm{Ch}(X)>0$, there are no $H$-spheres in $Y$ for $0<H \leq \mathrm{Ch}(X) / 2$.

3. The space $\mathcal{M}_{H}(Y)$ of all immersed $H$-spheres in $Y$ for a given $H>$ $\mathrm{Ch}(X) / 2$ is naturally diffeomorphic to $Y$ (by the map that sends every $S_{H}$ into $p\left(S_{H}\right)$ ), where $H$-spheres in $Y$ with distinct images are considered to be different elements in $\mathcal{M}_{H}(Y)$.

4. Any $H$-sphere in $Y$ for $H>0$ has index one and nullity three for its stability operator.

5. Any $H$-sphere $S_{H}$ in $Y$ for $H>0$ is invariant under every isometry of $Y$ that fixes $p\left(S_{H}\right)$.

6. Given any two $H$-spheres $S_{H}^{0}, S_{H}^{1} \in \mathcal{M}_{H}(Y)$ for some $H>0$, there exist an isometry $\phi: S_{H}^{0} \rightarrow S_{H}^{1}$ that also preserves the second fundamental forms of $S_{H}^{0}$ and $S_{H}^{1}$ at corresponding points. Moreover, given any path $\alpha:[0,1] \rightarrow Y$ joining $p\left(S_{H}^{0}\right)$ to $p\left(S_{H}^{1}\right)$, there is an associated family of isometric $H$-spheres $S_{H}(t), t \in[0,1]$, in $Y$ with $S_{H}^{0}=S_{H}(0)$ and $S_{H}^{1}=S_{H}(1)$, such that $p\left(S_{H}(t)\right)=\alpha(t)$ for every $t$. 
We observe that item 6 above is the natural generalization to complete locally homogeneous three-manifolds of the uniqueness result in Theorem 1.1 that any two spheres of the same constant mean curvature in a homogeneous three-manifold differ by an ambient isometry.

\section{Background on constant mean curvature surface theory in metric Lie groups}

In this section we will collect some basic material about metric Lie groups diffeomorphic to $\mathbb{R}^{3}$ and immersed surfaces of constant mean curvature in these spaces, that will be needed in later sections. For the purposes of this paper, it is worth dividing metric Lie groups $X$ diffeomorphic to $\mathbb{R}^{3}$ into two classes depending on whether the underlying group structure of $X$ is that of a semidirect product, or it is the one of $\widetilde{\mathrm{SL}}(2, \mathbb{R})$, that can be naturally identified as the universal cover of the group $\operatorname{PSL}(2, \mathbb{R})$ of orientation-preserving isometries of the hyperbolic plane.

We divide this section into three parts: in Sect. 4.1 we describe some geometric aspects of constant mean curvature surfaces in a metric Lie group $X$ that are invariant under the flow of a nonzero right invariant vector field on $X$. Sections 4.2 and 4.3 describe the ambient geometry and particular aspects of surface theory in a metric Lie group isomorphic to a semidirect product or to $\widetilde{S L}(2, \mathbb{R})$, respectively. We will use the contents of Sect. 4.1 in Sect. 5 , but Sects. 4.2 and 4.3 will not be needed until Sect. 6. The basic references for this material are Milnor [34], the notes [28] and the paper [26].

\subsection{Invariant constant mean curvature surfaces}

Given a unit vector $v \in T_{e} X$ in the Lie algebra of $X$, we can extend $v$ by left translation to a left-invariant unit vector field $V$ globally defined on $X$. By using right translations in $X$ in a similar way, we can extend $v$ to a rightinvariant vector field $K$ in $X$. The vector field $K$ will not be, in general, of unit length anymore, but it is a nowhere-zero Killing field on $X$.

By [28, Corollary 3.17], left cosets of two-dimensional subgroups of $X$ are characterized by their left invariant Gauss map being constant, and they all have constant mean curvature with absolute value at most $H(X)$. As every right coset $\Lambda x$ of a two-dimensional subgroup $\Lambda$ of $X$ is the left coset of a conjugate subgroup of $\Lambda$ (namely $\Lambda x=x \Lambda_{1}$ where $\Lambda_{1}=x^{-1} \Lambda x$ ), then we deduce:

(C) The left invariant Gauss map of a left or right coset of a two-dimensional subgroup of $X$ is constant. 
The next proposition is a reformulation of Corollary 3.8 in [26]. We remark that Corollary 3.8 in [26] was written in terms of the $H$-potential of $X$, a concept that we will not introduce here, but one can easily translate that formulation into the one below by using [28, Corollary 3.21].

Proposition 4.1 Let $f: \Sigma \uparrow X$ be a complete immersed $H$-surface in $X$ and let $G: \Sigma \rightarrow \mathbb{S}^{2} \subset T_{e} X$ be its left invariant Gauss map. Assume that there are no two-dimensional subgroups in $X$ with mean curvature $H$ whose (constant) left invariant Gauss map lies in the Gauss map image $G(\Sigma)$. Then:

(1) The differential $d G$ of $G$ has rank at most 1 everywhere on $\Sigma$ if and only if $f$ is invariant under the flow of a nonzero right invariant vector field $K$ on $X$.

(2) If $f$ is invariant under the flow of a nonzero right invariant vector field of $X$, then $\operatorname{rank}(d G)=1$ everywhere on $\Sigma$, and $G(\Sigma)$ is a regular curve in $\mathbb{S}^{2}$.

Remark 4.2 If $K$ is a nonzero right invariant vector field on a metric Lie group $X$ diffeomorphic to $\mathbb{R}^{3}$, then each integral curve of $K$ is diffeomorphic to $\mathbb{R}$ and the quotient space $X / K$ of integral curves of $K$ inherits a two-dimensional differentiable structure that makes the natural projection $\Pi_{K}: X \rightarrow X / K$ a submersion with $\operatorname{ker}\left[d\left(\Pi_{K}\right)_{x}\right]=\operatorname{Span}\{K(x)\}$ for all $x \in X$. Therefore, when $f: \Sigma \leftrightarrow \rightarrow$ satisfies the hypothesis in item 2 of Proposition 4.1, we can consider $f(\Sigma)$ as the surface in $X$ obtained by pulling back via $\Pi_{K_{\Sigma}}$ an immersed curve $\beta$ contained in $X / K_{\Sigma}$. Moreover, after identifying in the standard way $X / K_{\Sigma}$ with any entire Killing graph $S_{0} \subset X$ with respect to $K_{\Sigma}$, the invariant surface $f: \Sigma \rightarrow X$ can be parameterized locally (and even globally if $\Sigma$ is simply connected) as

$$
f(s, t)=l_{\Gamma(t)}(\beta(s)), \quad(s, t) \in I \times \mathbb{R},
$$

where $\beta(s): I \subset \mathbb{R} \rightarrow X / K_{\Sigma} \equiv S_{0}$ is the immersed curve above, and $\Gamma=\Gamma(t)$ is the 1-parameter subgroup of $X$ given by $\Gamma^{\prime}(0)=K_{\Sigma}(e)$.

Let $f: \Sigma \rightarrow X$ be an immersed $H$-surface in $X$ whose left invariant Gauss map $G: \Sigma \rightarrow \mathbb{S}^{2}$ has rank one at every point. It is shown in [26, proof of Corollary 3.8] that around any $z_{0} \in \Sigma$ there exist conformal parameters $(s, t)$ on $\Sigma$ such that $G$ does not depend on $t$, so we can write $G=G(s)$. With respect to these coordinates, the Gauss map $G(s)$ satisfies a second order autonomous ODE of the form

$$
G^{\prime \prime}=\Psi\left(G, G^{\prime}\right)
$$


where $\Psi: \mathbb{S}^{2} \times \mathbb{R}^{3} \rightarrow \mathbb{R}^{3}$ is real analytic; this follows directly from the ODE (4.3) in [26], which is of the form

$$
\widehat{g}^{\prime \prime}=A(\widehat{g})\left(\widehat{g}^{\prime}\right)^{2}+B(\widehat{g})\left|\widehat{g}^{\prime}\right|^{2},
$$

where $A, B$ are real analytic. Here, $\widehat{g}=\pi \circ G$, where $\pi: \mathbb{S}^{2} \rightarrow \overline{\mathbb{C}}$ is a suitable stereographic projection with respect to an orthonormal basis of the Lie algebra of $X$. The special form of this ODE has the following trivial consequence:

(D) If $\widehat{g}(s)$ is a solution of (4.2), then $\widehat{g}\left(\delta_{1} s+\delta_{2}\right), \delta_{1} \neq 0$, is also a solution of the same ODE. Consequently, the same property holds for a solution $G(s)$ of (4.1).

Lemma 4.3 Let $f_{i}: \Sigma_{i} \rightarrow X, i=1,2$ denote two complete immersed $H$ surfaces in $X$ whose Gauss map images $\gamma_{i}:=G_{i}\left(\Sigma_{i}\right)$ are regular curves in $\mathbb{S}^{2}$. Assume that there exist points $p_{i} \in \Sigma_{i}$ with $f_{1}\left(p_{1}\right)=f_{2}\left(p_{2}\right)$ and $G_{1}\left(p_{1}\right)=G_{2}\left(p_{2}\right)=: v \in \mathbb{S}^{2}$, so that $\gamma_{1}, \gamma_{2}$ intersect tangentially at $v$. Then $f_{1}\left(\Sigma_{1}\right)=f_{2}\left(\Sigma_{2}\right)$.

Proof By the previous comments, we can view $f_{1}, f_{2}$ locally around $p_{1}, p_{2}$ as conformal immersions $f_{i}(s, t)$ into $X$, and $G_{1}, G_{2}$ as regular parameterized curves $G_{i}(s): J_{i} \rightarrow \mathbb{S}^{2}$, both of them satisfying the ODE (4.1) on an open interval $J_{i} \subset \mathbb{R}$. The conditions in the statement of the lemma imply that $G_{1}\left(s_{1}\right)=G_{2}\left(s_{2}\right)$ and $\left(G_{1}\right)^{\prime}\left(s_{1}\right)= \pm\left(G_{2}\right)^{\prime}\left(s_{2}\right)$ for some $s_{i} \in J_{i}$. But once here, property (D) above and the uniqueness of solution to the Cauchy problem for ODEs imply that $G_{1}(s)=G_{2}\left(\delta_{1} s+\delta_{2}\right)$ for adequate constants $\delta_{1} \neq 0$ and $\delta_{2}$. In particular, the two Gauss maps coincide after conformal reparameterization of $f_{1}$ or $f_{2}$. Recall now that, by [26, Theorem 3.7], the Gauss map of a conformally parameterized $H$-surface in $X$ determines the surface up to left translation in $X$. Since $f_{1}\left(p_{1}\right)=f_{2}\left(p_{2}\right)$, this left translation is trivial in our case, and so we conclude that $f_{1}\left(\Sigma_{1}\right)=f_{2}\left(\Sigma_{2}\right)$.

\subsection{Metric semidirect products}

Given a real $2 \times 2$ matrix $A \in \mathcal{M}_{2}(\mathbb{R})$, the semidirect product $\mathbb{R}^{2} \rtimes_{A} \mathbb{R}$ is the Lie group $\left(\mathbb{R}^{3} \equiv \mathbb{R}^{2} \times \mathbb{R}, *\right)$ endowed with the group operation $\left(\mathbf{p}_{1}, z_{1}\right) *\left(\mathbf{p}_{2}, z_{2}\right)=$ $\left(\mathbf{p}_{1}+e^{z_{1} A} \mathbf{p}_{2}, z_{1}+z_{2}\right)$; here $e^{B}=\sum_{k=0}^{\infty} \frac{1}{k !} B^{k}$ denotes the usual exponentiation of a matrix $B \in \mathcal{M}_{2}(\mathbb{R})$. Let

$$
A=\left(\begin{array}{ll}
a & b \\
c & d
\end{array}\right), \quad e^{z A}=\left(\begin{array}{ll}
a_{11}(z) & a_{12}(z) \\
a_{21}(z) & a_{22}(z)
\end{array}\right) .
$$

Then, a left invariant frame $\left\{E_{1}, E_{2}, E_{3}\right\}$ of $X$ is given by

$$
E_{1}(x, y, z)=a_{11}(z) \partial_{x}+a_{21}(z) \partial_{y},
$$




$$
E_{2}(x, y, z)=a_{12}(z) \partial_{x}+a_{22}(z) \partial_{y}, \quad E_{3}=\partial_{z} .
$$

Observe that $\left\{E_{1}, E_{2}, E_{3}\right\}$ is the left invariant extension of the canonical basis $\left(\partial_{x}\right)_{e},\left(\partial_{y}\right)_{e},\left(\partial_{z}\right)_{e}$ of the tangent space $T_{e} X$ at the identity element $e=(0,0,0)$. The right invariant extensions on $X$ of the same vectors of $T_{e} X$ define the frame $F_{1}, F_{2}, F_{3}$ where

$$
\begin{aligned}
& F_{1}=\partial_{x}, \quad F_{2}=\partial_{y}, \\
& F_{3}(x, y, z)=(a x+b y) \partial_{x}+(c x+d y) \partial_{y}+\partial_{z} .
\end{aligned}
$$

In terms of $A$, the Lie bracket relations are:

$$
\left[E_{1}, E_{2}\right]=0, \quad\left[E_{3}, E_{1}\right]=a E_{1}+c E_{2}, \quad\left[E_{3}, E_{2}\right]=b E_{1}+d E_{2} .
$$

Definition 4.4 We define the canonical left invariant metric on $\mathbb{R}^{2} \rtimes_{A} \mathbb{R}$ to be that one for which the left invariant frame $\left\{E_{1}, E_{2}, E_{3}\right\}$ given by (4.4) is orthonormal. Equivalently, it is the left invariant extension to $X=\mathbb{R}^{2} \rtimes_{A} \mathbb{R}$ of the inner product on $T_{e} X$ that makes $\left(\partial_{x}\right)_{e},\left(\partial_{y}\right)_{e},\left(\partial_{z}\right)_{e}$ an orthonormal basis.

Some basic properties of the canonical left invariant metric $\langle$,$\rangle on \mathbb{R}^{2} \rtimes_{A} \mathbb{R}$ are the following ones.

(E1) The vector fields $F_{1}, F_{2}, F_{3}$ are Killing.

(E2) The mean curvature of each leaf of the foliation $\mathcal{F}=\left\{\mathbb{R}^{2} \rtimes_{A}\{z\} \mid\right.$ $z \in \mathbb{R}\}$ with respect to the unit normal vector field $E_{3}$ is the constant $H=\operatorname{trace}(A) / 2$. All the leaves of the foliation $\mathcal{F}$ are intrinsically flat, and have critical constant mean curvature. Moreover, the Gauss map image of each leaf of $\mathcal{F}$ with respect to the orientation given by $E_{3}$ (resp. $-E_{3}$ ) is the North (resp. South) pole of $\mathbb{S}^{2}$ with respect to the left invariant frame $\left\{E_{1}, E_{2}, E_{3}\right\}$.

(E3) The change (4.4) from the orthonormal basis $\left\{E_{1}, E_{2}, E_{3}\right\}$ to the basis $\left\{\partial_{x}, \partial_{y}, \partial_{z}\right\}$ produces the following expression of $\langle$,$\rangle in the x, y, z$ coordinates of $X$ :

$$
\begin{aligned}
\langle,\rangle= & {\left[a_{11}(-z)^{2}+a_{21}(-z)^{2}\right] d x^{2} } \\
& +\left[a_{12}(-z)^{2}+a_{22}(-z)^{2}\right] d y^{2}+d z^{2} \\
& +\left[a_{11}(-z) a_{12}(-z)+a_{21}(-z) a_{22}(-z)\right] \\
& (d x \otimes d y+d y \otimes d x) .
\end{aligned}
$$

(E4) The $\pi$-rotation about any of the integral curves of $\partial_{z}=E_{3}$ (vertical lines in the $(x, y, z)$-coordinates) is an order-two orientation preserving isometry. 
In what follows, we will use the standard notation $\mathbb{E}(\kappa, \tau)$ (as in [8]) for a simply connected homogeneous three-manifold $X$ with an isometry group of dimension 4; see Sect. 3. As in our situation $X$ is diffeomorphic to $\mathbb{R}^{3}$, then by classification $\kappa \leq 0$ and $X$ is isometric to $\mathbb{H}^{2}(\kappa) \times \mathbb{R}$ (if $\tau=0, \kappa<0$ ), to Heisenberg space $\mathrm{Nil}_{3}$ (if $\tau \neq 0, \kappa=0$ ), or to $\widetilde{\mathrm{SL}}(2, \mathbb{R}$ ) with a rotationally symmetric left invariant metric if $\tau \neq 0, \kappa<0$.

Lemma 4.5 If $A$ is a singular matrix, then $X=\mathbb{R}^{2} \rtimes_{A} \mathbb{R}$ is isometric to $\mathbb{R}^{3}$ or to an $\mathbb{E}(\kappa, \tau)$-space with $\kappa \leq 0$ and $\tau \in \mathbb{R}$.

Proof If $A=0$, then $X$ is the standard $\mathbb{R}^{3}$. If $A \neq 0$ and $\operatorname{trace}(A)=0$, then [28, Theorem 2.15] gives that either $\operatorname{det}(A) \neq 0$ (which contradicts our hypothesis) or $X$ is isometric to the Heisenberg space $\mathrm{Nil}_{3}$ (recall that $\mathrm{Nil}_{3}$ admits a 1-parameter family of homogeneous metrics, all of which are homothetic). Finally, if trace $(A) \neq 0$ then $X$ is isometric to some $\mathbb{E}(\kappa, \tau)$ with $\kappa<0$ and $\tau \in \mathbb{R}$ as explained in [28, Section 2.8].

Remark 4.6 Let us explain the relation between the basic geometric properties of $\mathbb{E}(\kappa, \tau)$ when viewed as a fibration $\pi: \mathbb{E}(\kappa, \tau) \rightarrow \mathbb{M}^{2}(\kappa)$, and when viewed as a metric semidirect product $\mathbb{R}^{2} \rtimes_{A} \mathbb{R}$, with $A$ singular. When trace $(A) \neq 0$ (with $A$ singular) we have, as explained in the proof of the above lemma, that $\kappa<0$. In that situation, one can check that the unit left invariant vector field $E_{2}$ in (4.4) is actually equal to the Killing field $F_{2}=\partial_{y}$ in (4.5). Thus, $E_{2}$ is actually the unit Killing vertical field associated to the canonical fibration of $\mathbb{E}(\kappa, \tau)$; see the proof of [28, Theorem 2.15]. With this, the family of horizontal planes $\mathbb{R}^{2} \rtimes_{A}\{z\}, z \in \mathbb{R}$, in the semidirect product model corresponds to a family of parallel horocylinders of $\mathbb{E}(\kappa, \tau)$ in the fibration model.

In the case that trace $(A)=0$ (with $A$ singular, and non-zero), $X$ is isometric to $\mathbb{E}(0, \tau) \equiv \mathrm{Nil}_{3}$, for some $\tau \neq 0$. This time, the vector field $E_{1}$ in (4.4) corresponds to the unit Killing field associated to the fibration model of $X$, and the horizontal planes $\mathbb{R}^{2} \rtimes_{A}\{z\}, z \in \mathbb{R}$, correspond to a family of vertical, parallel minimal planes in the usual $\mathbb{E}(\kappa, \tau)$ model of Heisenberg space.

A simple consequence of Eqs. (4.5) and (4.6) is that every horizontal right invariant vector field (i.e., every linear combination of $\partial_{x}, \partial_{y}$ with constant coefficients) is bounded in $\mathbb{R}^{2} \rtimes_{A}\left[z_{1}, z_{2}\right]$, for all $z_{1}, z_{2} \in \mathbb{R}$ with $z_{1} \leq z_{2}$. The following lemma guarantees the existence of at least one nonzero horizontal right invariant vector field in $\mathbb{R}^{2} \rtimes_{A} \mathbb{R}$ which is bounded in a horizontal halfspace.

Lemma 4.7 Let $X$ be a semidirect product $\mathbb{R}^{2} \rtimes_{A} \mathbb{R}$ endowed with its canonical metric, where $A \in \mathcal{M}_{2}(\mathbb{R})$. Then, there exists a nonzero horizontal right invariant vector field which is bounded in $\mathbb{R}^{2} \rtimes_{A}[0, \infty)$. 
Proof If trace $(A) \neq 0$, the lemma follows from [25, item 2 of Proposition 6.1]. If trace $(A)=0$, then either $A=0$ and $\mathbb{R}^{2} \rtimes_{A} \mathbb{R}$ is the standard Euclidean space, for which the lemma is obvious, or else $A \neq 0$ and [28, item 2 of Theorem 2.15] implies that there are three possible cases for $A$ up to rescaling the metric. We will prove the lemma by studying each case separately.

- $A=\left(\begin{array}{cc}0 & -c \\ 1 / c & 0\end{array}\right)$ for some $1 \leq c<\infty$. This case corresponds to the group structure of $\widetilde{\mathrm{E}}(2)$, the universal cover of the group of orientation-preserving rigid motions of the Euclidean plane. A direct computation using (4.5) and (4.6) gives $\left|F_{1}\right|^{2}=\cos ^{2} z+c^{-2} \sin ^{2} z$ and $\left|F_{2}\right|^{2}=\cos ^{2} z+c^{2} \sin ^{2} z$, both bounded.

- $A=\left(\begin{array}{cc}0 & c \\ 1 / c & 0\end{array}\right)$ for some $1 \leq c<\infty$. In this case, $X$ is isomorphic to $\mathrm{Sol}_{3}$. A similar reasoning as in the preceding case gives that the basis of horizontal right invariant vector fields $\widehat{F}_{1}=-c \partial_{x}+\partial_{y}, \widehat{F}_{2}=c \partial_{x}+\partial_{y}$ satisfies $\left|\widehat{F}_{1}\right|=\sqrt{1+c^{2}} e^{z},\left|\widehat{F}_{2}\right|=\sqrt{1+c^{2}} e^{-z}$.

- $A=\left(\begin{array}{ll}0 & 1 \\ 0 & 0\end{array}\right)$ and $X$ is isomorphic to the Heisenberg space Nil 3 . Using again (4.5) and (4.6) we have that $\left|F_{1}\right|=1$.

\subsection{Metric Lie groups isomorphic to $\widetilde{\mathrm{SL}}(2, \mathbb{R})$}

The Lie group $\widetilde{\mathrm{SL}}(2, \mathbb{R})$ is the universal cover of the special linear group $\operatorname{SL}(2, \mathbb{R})=\left\{A \in \mathcal{M}_{2}(\mathbb{R}) \mid \operatorname{det} A=1\right\}$, and of the projective special linear group $\operatorname{PSL}(2, \mathbb{R})=\operatorname{SL}(2, \mathbb{R}) /\left\{ \pm I_{2}\right\}$. The Lie algebra of any of the groups $\widetilde{\mathrm{SL}}(2, \mathbb{R}), \operatorname{SL}(2, \mathbb{R})$, and $\operatorname{PSL}(2, \mathbb{R})$ is $\mathfrak{s l}(2, \mathbb{R})=\left\{B \in \mathcal{M}_{2}(\mathbb{R}) \mid \operatorname{trace}(B)=\right.$ $0\}$. It is worth recalling that $\operatorname{PSL}(2, \mathbb{R})$ has several isomorphic models, that we will use in the sequel:

(F1) The group of orientation-preserving isometries of the hyperbolic plane,

$$
z \in \mathbb{H}^{2} \equiv\left(\mathbb{R}^{2}\right)^{+} \mapsto \frac{a z+b}{c z+d} \in\left(\mathbb{R}^{2}\right)^{+} \quad(a, b, c, d \in \mathbb{R}, a d-b c=1) .
$$

(F2) The group of conformal automorphisms of the unit disc $\mathbb{D}:=\{z \in \mathbb{C}$ : $|z|<1\}$; these automorphisms are the Möbius transformations of the type $\phi(z)=\xi \frac{z+a}{1+\bar{a} z}$, with $a \in \mathbb{D}$ and $|\xi|=1$. The universal cover map $\theta \mapsto \xi:=e^{i \theta}: \mathbb{R} \rightarrow \mathbb{S}^{1}$ gives $\widetilde{\mathrm{SL}}(2, \mathbb{R})$ a structure of an $\mathbb{R}$-bundle over $\mathbb{D}$.

(F3) The unit tangent bundle of $\mathbb{H}^{2}$. This interpretation of $\operatorname{PSL}(2, \mathbb{R})$ as an $\mathbb{S}^{1}$-bundle over $\mathbb{H}^{2}$ (and of $\widetilde{\mathrm{SL}}(2, \mathbb{R})$ as an $\mathbb{R}$-bundle over $\mathbb{H}^{2}$ ) produces a submersion from $\widetilde{\mathrm{SL}}(2, \mathbb{R})$ to $\mathbb{H}^{2}$. 
There are three types of 1-parameter subgroups of $\widetilde{\mathrm{SL}}(2, \mathbb{R})$ :

- Elliptic subgroups. Elements of any of these subgroups correspond to liftings to $\widetilde{\mathrm{SL}}(2, \mathbb{R})$ of rotations around any fixed point in $\mathbb{H}^{2}$. These groups of rotations fix no points in the boundary at infinity $\partial_{\infty} \mathbb{H}^{2}$. Any two elliptic 1-parameter subgroups are conjugate.

- Hyperbolic subgroups. These subgroups correspond to liftings to $\widetilde{\operatorname{SL}}(2, \mathbb{R})$ of translations along any fixed geodesic in $\mathbb{H}^{2}$. In the Poincaré disk model of $\mathbb{H}^{2}$, the hyperbolic subgroup associated to a geodesic $\Gamma$ fixes the two points at infinity given by the end points of $\Gamma$. As in the elliptic case, any two 1-parameter hyperbolic subgroups are conjugate.

- Parabolic subgroups. They correspond to liftings to $\widetilde{\mathrm{SL}}(2, \mathbb{R})$ of rotations about any fixed point $\theta \in \partial_{\infty} \mathbb{H}^{2}$. Any of these rotation subgroups only fixes the point $\theta$ at infinity, and leaves invariant the 1-parameter family of horocycles based at $\theta$. As in the previous cases, 1-parameter parabolic subgroups are all conjugate by elliptic rotations of the Poincaré disk.

The character of a 1-parameter subgroup $\Gamma$ of $\widetilde{\mathrm{SL}}(2, \mathbb{R})$ refers to the property of $\Gamma$ being elliptic, parabolic or hyperbolic. The character is invariant by conjugation, i.e., if $\Gamma$ is a 1-parameter subgroup of $\widetilde{\mathrm{SL}}(2, \mathbb{R})$ and we define $\Gamma_{a}:=a \Gamma a^{-1}=l_{a}\left(r_{a^{-1}}(\Gamma)\right)$ for some $a \in \widetilde{\mathrm{SL}}(2, \mathbb{R})$, then $\Gamma_{a}$ has the same character as $\Gamma$.

Nonzero right invariant vector fields $K$ are in one-to-one correspondence with 1-parameter subgroups $\Gamma$ and with tangent vectors at the identity element $e$ of $\widetilde{\mathrm{SL}}(2, \mathbb{R})$ by the formula $K_{e}=\Gamma^{\prime}(0)$. We will say that a nonzero right invariant vector field $K$ on $\widetilde{\mathrm{SL}}(2, \mathbb{R})$ is elliptic (resp. hyperbolic, parabolic) when the related 1-parameter subgroup $\Gamma$ in $\widetilde{\mathrm{SL}}(2, \mathbb{R})$ is elliptic (resp. hyperbolic, parabolic).

The matrices in the Lie algebra $\mathfrak{s l}(2, \mathbb{R})$ given by

$$
\left(E_{1}\right)_{e}=\left(\begin{array}{rr}
1 & 0 \\
0 & -1
\end{array}\right), \quad\left(E_{2}\right)_{e}=\left(\begin{array}{ll}
0 & 1 \\
1 & 0
\end{array}\right), \quad\left(E_{3}\right)_{e}=\left(\begin{array}{rr}
0 & -1 \\
1 & 0
\end{array}\right)
$$

define a left invariant frame $\left\{E_{1}, E_{2}, E_{3}\right\}$ on $\widetilde{\mathrm{SL}}(2, \mathbb{R})$ with the property that $\left[E_{1}, E_{2}\right]=-2 E_{3},\left[E_{2}, E_{3}\right]=2 E_{1},\left[E_{3}, E_{1}\right]=2 E_{2}$. The fields $E_{1}, E_{2}, E_{3}$ are respectively generated by the 1-parameter subgroups of $\operatorname{SL}(2, \mathbb{R})$ given by

$$
\left(\begin{array}{cc}
e^{t} & 0 \\
0 & e^{-t}
\end{array}\right), \quad\left(\begin{array}{cc}
\cosh t & \sinh t \\
\sinh t & \cosh t
\end{array}\right), \quad\left(\begin{array}{cc}
\cos t & -\sin t \\
\sin t & \cos t
\end{array}\right), \quad t \in \mathbb{R}
$$

where the first two are hyperbolic subgroups and the third one is an elliptic subgroup. 
The center $Z$ of $\widetilde{\mathrm{SL}}(2, \mathbb{R})$ is an infinite cyclic subgroup (hence isomorphic to $\mathbb{Z})$ whose elements correspond to liftings to $\widetilde{\mathrm{SL}}(2, \mathbb{R})$ of rotations by angles that are multiples of $2 \pi$; if $\Lambda: \widetilde{\operatorname{SL}}(2, \mathbb{R}) \rightarrow \operatorname{PSL}(2, \mathbb{R})$ denotes the universal covering map, then $Z=\Lambda^{-1}(e)$ where $e$ is the identity element of $\operatorname{PSL}(2, \mathbb{R})$. In this way, $Z$ is contained in the integral curve $\Gamma_{3} \subset \widetilde{\mathrm{SL}}(2, \mathbb{R})$ of the left invariant vector field $E_{3}$ given by (4.8) (indeed, $Z$ is contained in every elliptic subgroup of $\widetilde{\mathrm{SL}}(2, \mathbb{R})$ ).

By declaring the left invariant frame $\left\{E_{1}, E_{2}, E_{3}\right\}$ to be orthonormal, we define a left invariant metric $\langle$,$\rangle on \widetilde{\mathrm{SL}}(2, \mathbb{R})$ such that the metric Lie group $(\widetilde{\mathrm{SL}}(2, \mathbb{R}),\langle\rangle$,$) is isometric to the \mathbb{E}(\kappa, \tau)$-space with base curvature $\kappa=-4$ and bundle curvature $\tau^{2}=1$. Let

$$
\Pi_{0}: \mathbb{E}(-4,1) \rightarrow \mathbb{H}^{2}(-4)
$$

be the associated Riemannian submersion onto the hyperbolic plane endowed with the metric of constant curvature -4 (this submersion can be naturally identified with any of the submersions described in properties (F2) and (F3) above). Let $\phi:(\widetilde{\mathrm{SL}}(2, \mathbb{R}),\langle\rangle,) \rightarrow \mathbb{E}(-4,1)$ be a Riemannian isometry. Then, the image by $\phi$ of every integral curve of $E_{3}$ is a $\Pi_{0}$-vertical geodesic. The composition

$$
\Pi=\Pi_{0} \circ \phi: \widetilde{\mathrm{SL}}(2, \mathbb{R}) \rightarrow \mathbb{H}^{2}(-4)
$$

defines a submersion which is Riemannian with respect to the particular left invariant metric $\langle$,$\rangle defined above.$

The next result follows from [28, Section 2.7] and describes the geometry of each 1-parameter subgroup of $\widetilde{\mathrm{SL}}(2, \mathbb{R})$ in terms of the coordinates of its velocity vector at the identity element $e$ with respect to the basis $\left\{E_{1}, E_{2}, E_{3}\right\}$.

Lemma 4.8 Consider a vector $\Gamma^{\prime}(0) \in T_{e} \widetilde{\mathrm{SL}}(2, \mathbb{R})$ with coordinates $(a, b, c)$ with respect to the basis given in (4.7), and let $\Gamma$ denote the 1-parameter subgroup generated by $\Gamma^{\prime}(0)$. Then:

1. $\Gamma$ is elliptic if and only if $a^{2}+b^{2}<c^{2}$. If $a=b=0$, then $\Gamma$ is the lift to $\widetilde{\mathrm{SL}}(2, \mathbb{R})$ of the elliptic subgroup of rotations of $\mathbb{H}^{2}(-4)$ around the point $\Pi(e)$. If $0<a^{2}+b^{2}<c^{2}$, then $\Pi(\Gamma)$ is a constant geodesic curvature circle passing through $\Pi(e)$ and completely contained in $\mathbb{H}^{2}(-4)$.

2. $\Gamma$ is hyperbolic if and only if $a^{2}+b^{2}>c^{2}$. In this case, $\Pi(\Gamma)$ is a constant geodesic curvature arc passing through $\Pi(e)$ with two end points in the boundary at infinity of $\mathbb{H}^{2}(-4)$.

3. $\Gamma$ is parabolic if and only if $a^{2}+b^{2}=c^{2}$. In this case, then $\Pi(\Gamma)$ is a horocycle in $\mathbb{H}^{2}(-4)$.

Every left invariant metric in $\widetilde{\mathrm{SL}}(2, \mathbb{R})$ can be obtained by choosing numbers $\lambda_{1}, \lambda_{2}, \lambda_{3}>0$ and declaring the length of the left invariant vector field $E_{i}$ to 
be $\lambda_{i}, 1,2,3$, while keeping them orthogonal; see [27, Proposition 4.2]. For the remainder of this section, we will suppose that the metric Lie group $X$ is isometric and isomorphic to $\widetilde{\mathrm{SL}}(2, \mathbb{R})$ endowed with the left invariant metric given by an (arbitrary but fixed) choice of $\lambda_{i}>0, i=1,2,3$.

Lemma 4.9 Let $\gamma \subset \mathbb{H}^{2}(-4)$ be a geodesic. Denote by $\Sigma(\gamma)=\Pi^{-1}(\gamma)$ the surface in $\widetilde{\mathrm{SL}}(2, \mathbb{R})$ obtained by pulling back $\gamma$ through $\Pi$. Then:

(1) If $\Gamma \subset \widetilde{\mathrm{SL}}(2, \mathbb{R})$ is an integral curve of $E_{3}$, then $\phi(\Gamma)$ is an integral curve of the vertical vector field on $\mathbb{E}(-4,1)$ that generates the kernel of the differential of $\Pi_{0}$. Furthermore, if $R^{\Gamma}: \mathbb{E}(-4,1) \rightarrow \mathbb{E}(-4,1)$ denotes the rotation of angle $\pi$ around $\phi(\Gamma)$ (i.e., the lifting through $\Pi_{0}$ of the rotation $\psi_{\Pi(\Gamma)}$ by angle $\pi$ around the point $\Pi(\Gamma) \in \mathbb{H}^{2}(-4)$ ), then the map $R_{\Gamma}=\phi^{-1} \circ R^{\Gamma} \circ \phi$ is an order-two, orientation-preserving isometry of $X$ that fixes $\Gamma$.

(2) $\Sigma(\gamma)$ is ruled by geodesics of $X$ which are integral curves of $E_{3}$, and each integral curve $\Gamma$ of $E_{3}$ contained in $\Sigma(\gamma)$ is the fixed point set of the isometry $R_{\Gamma}: X \rightarrow X$, which restricts to an order-two, orientationreversing isometry on $\Sigma(\gamma)$.

(3) $\Sigma(\gamma)$ is a stable minimal surface in $X$.

(4) Given a curve $\gamma_{r} \subset \mathbb{H}^{2}(-4)$ at constant distance $r>0$ from $\gamma$, the surface $\Sigma\left(\gamma_{r}\right)=\Pi^{-1}\left(\gamma_{r}\right)$ has mean curvature vector of length bounded away from zero (not necessarily constant ${ }^{2}$ ) and pointing towards $\Sigma(\gamma)$.

(5) The critical mean curvature of $X$ (see Definition 3.5) satisfies $H(X) \geq \delta$, where $\delta$ is any positive lower bound for the mean curvature function of $\Sigma\left(\gamma_{r}\right)$ for any given $r>0$.

Proof Item 1 is well-known and follows from the fact that $E_{3}$ is a principal Ricci curvature direction at every point in $X$; see [28, Proposition 2.21] for details.

To prove item 2 , consider the integral curve $\Gamma \subset \widetilde{\mathrm{SL}}(2, \mathbb{R})$ of $E_{3}$ that passes through a given point $p \in \Sigma(\gamma)$. As $\Pi(\Gamma)=\Pi(p) \in \gamma$, then $\Gamma$ is entirely contained in $\Sigma(\gamma)$. Since $R^{\Gamma}: \mathbb{E}(-4,1) \rightarrow \mathbb{E}(-4,1)$ projects through $\Pi_{0}$ to the rotation $\psi_{\Pi(\Gamma)}: \mathbb{H}^{2}(-4) \rightarrow \mathbb{H}^{2}(-4)$ of angle $\pi$ around $\Pi(\Gamma) \in \gamma$, and $\psi_{\Pi(\Gamma)}$ leaves invariant the geodesic $\gamma$, then $R_{\Gamma}(\Sigma(\gamma))=\Sigma(\gamma)$. This proves that item 2 holds.

Minimality in item 3 follows since the mean curvature vector of $\Sigma(\gamma)$ maps to its negative under the differential of $R_{\Gamma} . \Sigma(\gamma)$ is stable because it admits a positive Jacobi function (namely, $\langle K, N\rangle$ where $K$ is the right invariant vector field on $\widetilde{\mathrm{SL}}(2, \mathbb{R})$ generated by the hyperbolic translations along a geodesic orthogonal to $\gamma$ in $\mathbb{H}^{2}(-4)$, and $N$ is a unit normal field to $\Sigma(\gamma)$ ).

2 The mean curvature of $\Pi_{0}^{-1}\left(\gamma_{r}\right) \subset \mathbb{E}(-4,1)$ is constant equal to half of the geodesic curvature of $\gamma_{r}$ in $\mathbb{H}^{2}(-4)$. 
To prove item 4, take a curve $\gamma_{r}$ at distance $r>0$ from a geodesic $\gamma \subset$ $\mathbb{H}^{2}(-4)$. Given a point $q \in \gamma_{r}$, there exists a geodesic $\widehat{\gamma} \subset \mathbb{H}^{2}(-4)$ passing through $q$ which is tangent to $\gamma_{r}$ at $q$, and $\widehat{\gamma}$ lies entirely on the side of $\gamma_{r}$ that does not contain $\gamma$. By item 3, the surface $\Sigma(\widehat{\gamma})=\Pi^{-1}(\widehat{\gamma})$ is minimal. Since $\Sigma(\widehat{\gamma})$ is tangent to $\Sigma\left(\gamma_{r}\right)$ and lies at one side of $\Sigma\left(\gamma_{r}\right)$, then the usual mean curvature comparison principle implies that the mean curvature of $\Sigma\left(\gamma_{r}\right)$ with respect to the unit normal vector field that points towards $\Sigma(\gamma)$, is nonnegative along $\Gamma=\Pi^{-1}(\{q\})$. In fact, the mean curvature of $\Sigma\left(\gamma_{r}\right)$ is strictly positive along $\Gamma$ as we explain next: otherwise the second fundamental forms $I I$ of $\Sigma\left(\gamma_{r}\right)$ and $\widehat{I I}$ of $\Sigma(\widehat{\gamma})$ would have the same trace and the same value in the direction given by the tangent vector $\Gamma^{\prime}$ to $\Gamma$; choosing $\Gamma^{\prime}$ as the first vector of an orthonormal basis $\mathcal{B}$ of the common tangent plane of both surfaces at any point of $\Gamma$, the matrix of the difference $I I-\widehat{I I}$ with respect to $\mathcal{B}$ is symmetric with zero diagonal entries; therefore, $I I-\widehat{I I}$ has opposite eigenvalues $a,-a$. If $a \neq 0$, then we contradict that $\Sigma(\widehat{\gamma})$ lies at one side of $\Sigma\left(\gamma_{r}\right)$; hence $I I=\widehat{I I}$ along $\Gamma$, which in turn implies that $\gamma_{r}$ would have a point of second order contact with $\widehat{\gamma}$, which is false. Therefore, the mean curvature function of $\Sigma\left(\gamma_{r}\right)$ is strictly positive.

To see that the mean curvature function of $\Sigma\left(\gamma_{r}\right)$ is bounded away from zero, consider the 1-parameter subgroup $G$ of hyperbolic translations of $\widetilde{\mathrm{SL}}(2, \mathbb{R})$ associated to the translations of $\mathbb{H}^{2}(-4)$ along $\gamma$. Then, $\Sigma\left(\gamma_{r}\right)$ is invariant under every element in $G$. Also, $\Sigma\left(\gamma_{r}\right)$ is invariant under left translation by every element in the center of $\widetilde{\mathrm{SL}}(2, \mathbb{R})$ (recall that elements in this center correspond to liftings to $\widetilde{S L}(2, \mathbb{R})$ of rotations by multiples of $2 \pi$ around a point in $\left.\mathbb{H}^{2}(-4)\right)$. Hence $\Sigma\left(\gamma_{r}\right)$ can be considered to be a doubly periodic surface. This property, together with the positivity of the mean curvature function of $\Sigma\left(\gamma_{r}\right)$, imply that item 4 holds.

Finally, item 5 is a direct consequence of item 4 and of the mean curvature comparison principle by the following argument. Let $\Lambda \subset X$ be any closed surface and take $r>0$. Since the mean convex side of $\Sigma\left(\gamma_{r}\right)$ contains arbitrarily large balls of $X$ in its interior, then after a left translation of $\Lambda$, we can assume that $\Lambda$ lies on the mean convex side of $\Sigma\left(\gamma_{r}\right)$. After further continuous left translations inside the mean convex side of $\Sigma\left(\gamma_{r}\right)$ applied to $\Lambda$, we can assume that $\Lambda$ is also tangent to $\Sigma\left(\gamma_{r}\right)$ at some common point $p$. By the mean curvature comparison principle, the absolute mean curvature function of $\Lambda$ at $p$ is at least as large as any lower positive bound $\delta$ of the positive mean curvature function of $\Sigma\left(\gamma_{r}\right)$. Hence, $H(X) \geq \delta$, which completes the proof.

Lemma 4.10 The push-forward by any left translation in $\widetilde{\mathrm{SL}}(2, \mathbb{R})$ of a right invariant elliptic (resp. hyperbolic, parabolic) vector field is again right invariant elliptic (resp. hyperbolic, parabolic) vector field. Furthermore, given a nonzero elliptic or parabolic vector field $F$ on $\widetilde{\mathrm{SL}}(2, \mathbb{R})$, the inner product of 
$F$ and the left invariant vector field $E_{3}$ given by (4.7), has constant nonzero sign on $\widetilde{\mathrm{SL}}(2, \mathbb{R})$, independently of the left invariant metric on $\widetilde{\mathrm{SL}}(2, \mathbb{R})$.

Proof The fact that the push-forward by a left translation of a right invariant vector field is again right invariant holds in any Lie group, and is well-known.

Consider now $F_{a}:=\left(l_{a}\right)_{*}(F)$, where $F$ is a right invariant vector field and $a \in \widetilde{\mathrm{SL}}(2, \mathbb{R})$. If $\Gamma$ denotes the 1 -parameter subgroup with $\Gamma^{\prime}(0)=F(e)$, then the corresponding 1-parameter subgroup $\Gamma_{a}$ that generates the right invariant vector field $F_{a}$ is given by $\Gamma_{a}=a \Gamma a^{-1}=l_{a}\left(r_{a^{-1}}(\Gamma)\right)$. In particular, $\Gamma_{a}$ and $\Gamma$ are conjugate subgroups, and so they have the same character. Thus, $F_{a}$ and $F$ also have the same character, as claimed.

Finally, observe that from the paragraph just before the statement of Lemma 4.9 we deduce that the sign of the inner product of a pair of tangent vectors $v, w \in T_{x} \widetilde{\mathrm{SL}}(2, \mathbb{R})$ at any $x \in X$ does not depend on the left invariant metric on $\widetilde{\mathrm{SL}}(2, \mathbb{R})$. Therefore, in order to prove the last sentence of the lemma, it suffices to choose a nonzero elliptic or parabolic vector field $F$ and prove that the function $\left\langle F, E_{3}\right\rangle: \widetilde{\mathrm{SL}}(2, \mathbb{R}) \rightarrow \mathbb{R}$ has no zeros, where $\langle$, is the metric defined just before (4.9).

Given $x \in \widetilde{\mathrm{SL}}(2, \mathbb{R})$, let $F^{x}$ be the vector field on $\widetilde{\mathrm{SL}}(2, \mathbb{R})$ defined by $\left(l_{x}\right)_{*}\left(F^{x}\right)=F$. By the first sentence of Lemma $4.10, F^{x}$ is a nonzero right invariant vector field of the same character as $F$ (i.e., elliptic or parabolic), and

$$
\left\langle F, E_{3}\right\rangle(x)=\left\langle\left(d l_{x}\right)_{e}\left(F_{e}^{x}\right),\left(E_{3}\right)_{x}\right\rangle=\left\langle F_{e}^{x},\left(E_{3}\right)_{e}\right\rangle=\left\langle F^{x}, E_{3}\right\rangle(e),
$$

where we have used that $E_{3}$ is left invariant and that $l_{x}$ is an isometry of $\langle$,$\rangle .$ As $F^{x}$ is elliptic or parabolic, by Lemma 4.8 , the coordinates $(a, b, c)$ of $F_{e}^{x}$ satisfy $a^{2}+b^{2} \leq c^{2}$, which implies that $\left\langle F, E_{3}\right\rangle(x)$ cannot vanish, as desired. Now the proof of Lemma 4.10 is complete.

We will finish this section with some considerations about two-dimensional subgroups of $\widetilde{\mathrm{SL}}(2, \mathbb{R})$ and their constant left invariant Gauss map images.

Given $\theta \in \partial_{\infty} \mathbb{H}^{2}$, let $\mathbb{H}_{\theta}^{2} \subset \widetilde{\mathrm{SL}}(2, \mathbb{R})$ be the set of liftings to $\widetilde{\mathrm{SL}}(2, \mathbb{R})$ of the orientation-preserving isometries of $\mathbb{H}^{2}$ that fix $\theta$. Elements in $\mathbb{H}_{\theta}^{2}$ are of two types: liftings to $\widetilde{\mathrm{SL}}(2, \mathbb{R})$ of hyperbolic translations along geodesics one of whose end points is $\theta$, and liftings to $\widetilde{\mathrm{SL}}(2, \mathbb{R})$ of parabolic rotations around $\theta . \mathbb{H}_{\theta}^{2}$ is a noncommutative, two-dimensional simply connected subgroup of $\widetilde{\mathrm{SL}}(2, \mathbb{R})$, and $\mathbb{H}_{\theta}^{2}$ contains a unique 1-parameter parabolic subgroup, namely the liftings to $\widetilde{\mathrm{SL}}(2, \mathbb{R})$ of parabolic rotations around $\theta$. Every two-dimensional subgroup of $\widetilde{\mathrm{SL}}(2, \mathbb{R})$ is of the form $\mathbb{H}_{\theta}^{2}$ for some $\theta \in \partial_{\infty} \mathbb{H}^{2}$, see the paragraph around equation (2.30) in [28] for more details.

Given a hyperbolic 1-parameter subgroup $\Gamma$ of $\widetilde{\mathrm{SL}}(2, \mathbb{R})$, by Lemma 4.8 , the image of $\Gamma$ through the map $\Pi$ defined in (4.10) is an arc in $\mathbb{H}^{2}(-4)$ of 

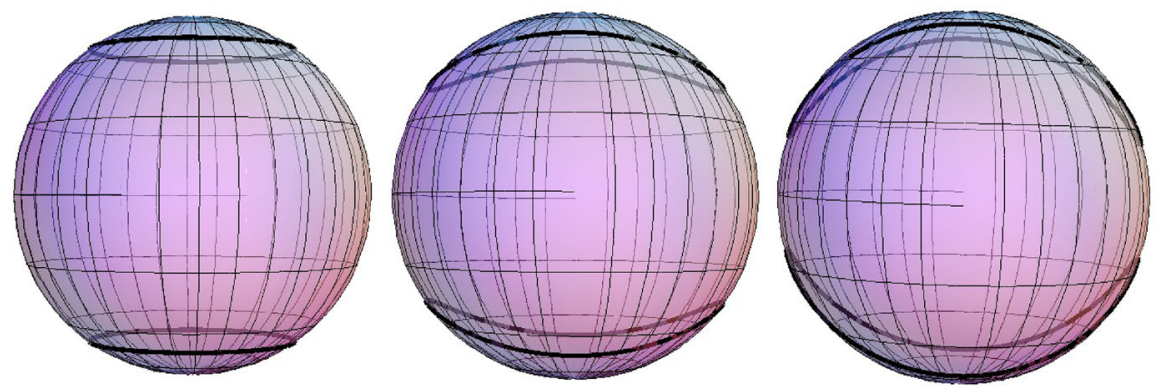

Fig. 1 Gauss map image of the family of two-dimensional subgroups $\mathbb{H}_{\theta}^{2}$ of $\widetilde{\mathrm{SL}}(2, \mathbb{R})$, with three different left invariant metrics. Left: $\lambda_{1}=\lambda_{2}=2, \lambda_{3}=1(\mathbb{E}(\kappa, \tau)$-metric). Center: $\lambda_{1}=0.5, \lambda_{2}=2, \lambda_{3}=1$. Right: $\lambda_{1}=0.1, \lambda_{2}=4, \lambda_{3}=1$

constant geodesic curvature that has two extrema $\theta_{1}, \theta_{2} \in \partial_{\infty} \mathbb{H}^{2}$. These two end-point values $\theta_{1}, \theta_{2}$ determine the two two-dimensional subgroups $\mathbb{H}_{\theta_{1}}^{2}$, $\mathbb{H}_{\theta_{2}}^{2}$ of $\widetilde{\mathrm{SL}}(2, \mathbb{R})$ that contain $\Gamma$.

The following result describes the image under the left invariant Gauss map of the circle family $\left\{\mathbb{H}_{\theta}^{2} \mid \theta \in \partial_{\infty} \mathbb{H}^{2}\right\}$ (recall that the left invariant Gauss map depends on the left invariant metric on $\widetilde{\mathrm{SL}}(2, \mathbb{R})$, and thus, it depends on the numbers $\lambda_{1}, \lambda_{2}, \lambda_{3}>0$ chosen just before Lemma 4.9).

Lemma 4.11 The constant value $G_{\theta} \in \mathbb{S}^{2}$ of the left invariant Gauss map of $\mathbb{H}_{\theta}^{2}$ is (up to a sign coming from a change of orientation):

$$
\begin{aligned}
G_{\theta}= & \frac{ \pm 1}{\sqrt{\lambda_{2} \lambda_{3} \sin ^{2} \theta+\lambda_{1} \lambda_{3} \cos ^{2} \theta+\lambda_{1} \lambda_{2}}} \\
& \left(-\sqrt{\lambda_{2} \lambda_{3}} \sin \theta, \sqrt{\lambda_{1} \lambda_{3}} \cos \theta,-\sqrt{\lambda_{1} \lambda_{2}}\right),
\end{aligned}
$$

in coordinates with respect to the orthonormal frame $\left\{\lambda_{i}^{-1 / 2}\left(E_{i}\right)_{e} \mid i=1,2,3\right\}$ (see Eq. (4.7)). The set $\left\{ \pm G_{\theta} \mid \theta \in \partial_{\infty} \mathbb{H}^{2}\right\}$ is a pair of antipodal, simple closed curves $\Upsilon \cup(-\Upsilon) \subset \mathbb{S}^{2}$, and it is invariant under the $\pi$-rotations in $\mathbb{S}^{2}$ in the directions of $\left(E_{1}\right)_{e},\left(E_{2}\right)_{e},\left(E_{3}\right)_{e}$, see Fig. 1.

Proof Let $v \in T_{e} \widetilde{\mathrm{SL}}(2, \mathbb{R})$ be a tangent vector with coordinates $(a, b, c)$ with respect to $\left\{\left(E_{1}\right)_{e},\left(E_{2}\right)_{e},\left(E_{3}\right)_{e}\right\}$. By Lemma 4.8, the tangent vector $v^{P} \in T_{e} \widetilde{\widetilde{S L}}(2, \mathbb{R})$ with coordinates $(a, b, c)=(-\sin \theta, \cos \theta, 1)$ (resp. $v^{H} \in T_{e} \widetilde{\mathrm{SL}}(2, \mathbb{R})$ with $\left.(a, b, c)=(\cos \theta, \sin \theta, 0)\right)$ produces a parabolic (resp. hyperbolic) 1-parameter subgroup $\Gamma^{P}$ (resp. $\Gamma^{H}$ ) of $\widetilde{\mathrm{SL}}(2, \mathbb{R})$. Furthermore, the projection of $\Gamma^{P}$ (resp. of $\Gamma^{H}$ ) through the map $\Pi$ defined in (4.10) is the horocycle that passes through the origin of the Poincaré disk and through the point at infinity $e^{i \theta} \in \partial_{\infty} \mathbb{H}^{2}$ (resp. the segment with extrema $\pm e^{i \theta}$ ). It 
follows that both subgroups $\Gamma^{P}, \Gamma^{H}$ are contained in the two-dimensional subgroup $\mathbb{H}_{\theta}^{2}$ of $\widetilde{\mathrm{SL}}(2, \mathbb{R})$.

The coordinates of $v^{P}, v^{H}$ with respect to the frame $\left\{\lambda_{i}^{-1 / 2}\left(E_{i}\right)_{e} \mid i=\right.$ $1,2,3\}$ are respectively $\left(-\sqrt{\lambda_{1}} \sin \theta, \sqrt{\lambda_{2}} \cos \theta, \sqrt{\lambda_{3}}\right)$ and $\left(\sqrt{\lambda_{1}} \cos \right.$ $\left.\theta, \sqrt{\lambda_{2}} \sin \theta, 0\right)$. After normalizing the cross product of these 3 -tuples, we deduce that the (constant) value of the left invariant Gauss map of $\mathbb{H}_{\theta}^{2}$ in the left invariant metric on $\widetilde{\mathrm{SL}}(2, \mathbb{R})$ determined by $\lambda_{1}, \lambda_{2}, \lambda_{3}>0$ is the one given by Eq. (4.11). The remaining conclusions of the lemma are direct consequences of (4.11).

\section{The geometry of invariant limit surfaces of index-one spheres}

In what follows, we will denote by $\mathcal{M}_{X}^{1}$ the moduli space of all index-one constant mean curvature spheres in a metric Lie group $X$ diffeomorphic to $\mathbb{R}^{3}$. Inside $\mathcal{M}_{X}^{1}$ we have the component $\mathcal{C}$ described in item 4 of Proposition 3.4. We will also use the number $h_{0}(X) \geq 0$ defined in Proposition 3.4.

Definition 5.1 We say that a complete, noncompact, connected $H$-surface $f: \Sigma \rightarrow X$ is a limit surface of $\mathcal{C}$ with base point $p \in \Sigma$ (also called a pointed limit immersion and denoted by $f:(\Sigma, p) \rightarrow(X, e))$ if $e=f(p)$ and there exists a sequence $\left\{\widehat{f}_{n}: S_{n} \rightarrow X\right\}_{n} \subset \mathcal{C}$, compact domains $\Omega_{n} \subset S_{n}$ and points $p_{n} \in \Omega_{n}$ such that the following two conditions hold:

(G1) $f$ is a limit as $n \rightarrow \infty$ of the immersions $f_{n}=l_{\widehat{f}_{n}\left(p_{n}\right)^{-1}} \circ\left(\left.\widehat{f}_{n}\right|_{\Omega_{n}}\right): \Omega_{n} \hookrightarrow \rightarrow$ $X$ obtained by left translating $\left.\widehat{f}_{n}\right|_{\Omega_{n}}$ by the inverse of $\widehat{f}_{n}\left(p_{n}\right)$ in $X$ (hence $f_{n}\left(p_{n}\right)=e$ ). Here, the convergence is the uniform convergence in the $C^{k}$-topology for every $k \geq 1$, when we view the surfaces as local graphs in the normal bundle of the limit immersion.

(G2) The area of $f_{n}$ is greater than $n$, for all $n \in \mathbb{N}$.

Remark 5.2 (1) By item 6 of Proposition 3.4, any such pointed limit immersion has constant mean curvature equal to $h_{0}(X)$. Moreover, by [26, Lemma 5.2], the space of pointed limit immersions of $\mathcal{C}$ is nonempty.

(2) If, with the notation in the last definition, $q_{n}$ is a point in $\Omega_{n}$ for each $n \in \mathbb{N}$ and the distance in $S_{n}$ from $q_{n}$ to $p_{n}$ is bounded independently of $n$, then

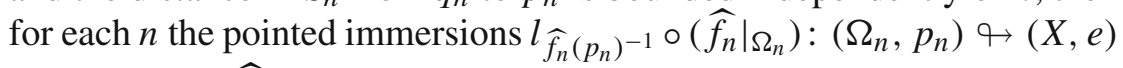
and $l_{\widehat{f}_{n}\left(q_{n}\right)^{-1}} \circ\left(\left.\widehat{f}_{n}\right|_{\Omega_{n}}\right):\left(\Omega_{n}, q_{n}\right) \leftrightarrow(X, e)$ differ only in the left translation in $X$ by the element $x_{n}:=\widehat{f}_{n}\left(q_{n}\right)^{-1} \widehat{f}_{n}\left(p_{n}\right)$. Observe that the distance in $X$ from $x_{n}$ to $e$ equals the distance on $X$ from $\widehat{f}_{n}\left(p_{n}\right)$ to $\widehat{f}_{n}\left(q_{n}\right)$, which is at most $d_{S_{n}}\left(p_{n}, q_{n}\right)$. In particular, if $d_{S_{n}}\left(p_{n}, q_{n}\right) \rightarrow 0$ as $n \rightarrow \infty$, we

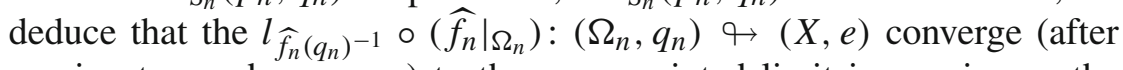
passing to a subsequence) to the same pointed limit immersion as the $l_{\widehat{f}_{n}\left(p_{n}\right)^{-1}} \circ\left(\left.\widehat{f}_{n}\right|_{\Omega_{n}}\right):\left(\Omega_{n}, p_{n}\right) \rightarrow(X, e)$. 
Frequently in this paper, we will apply the Transversality Lemma below. This is a basic property of immersed spheres in metric Lie groups proved by the authors in [24, Lemma 3.1], and that we formulate next adapted to our context.

Let $\Lambda$ be a two-dimensional subgroup of $X$. Then the set $\mathcal{F}=\left\{l_{a}(\Lambda)=\right.$ $a \Lambda \mid a \in X\}$ of left cosets of $\Lambda$ is a foliation of $X$. The quotient space $X / \Lambda$ is diffeomorphic to $\mathbb{R}$. Moreover, every element of $\mathcal{F}$ has the same constant Gauss map, say $g_{0} \in \mathbb{S}^{2}$, by property (C). With these considerations in mind, we have:

Lemma 5.3 (Transversality Lemma, [24]). Let $f: S \leftrightarrow X$ be an immersed sphere in $X$ whose left invariant Gauss map is a diffeomorphism. Then, with the notation above, $f(S)$ lies in the closed topological slab $\left[L_{a}, L_{b}\right]$ of $X$ determined by two leafs $L_{a}, L_{b}$ of $\mathcal{F}$, so that:

1. Each of the two leaves $L_{a}, L_{b}$ intersects $f(S)$ at a single point. At these two points, the Gauss map of $f$ is equal to $\pm g_{0}$.

2. Each leaf $L$ of $\mathcal{F}$ in the interior of $\left[L_{a}, L_{b}\right]$ intersects $f(S)$ transversely along a closed, connected, immersed curve. Moreover, $\alpha:=f^{-1}(L)$ is a closed, embedded curve in $S$.

Proposition 5.4 Let $f:(\Sigma, p) \rightarrow(X, e)$ be a pointed limit immersion of $\mathcal{C}$, and let $G: \Sigma \rightarrow \mathbb{S}^{2} \subset T_{e} X$ be its left invariant Gauss map. Then, the rank of the differential $d G$ is a constant $k \in\{0,1,2\}$ on $\Sigma$. Furthermore:

(1) If $\operatorname{rank}(d G)=0$, then $f$ is injective and $f(\Sigma)$ is a two-dimensional subgroup of $X$.

(2) If $\operatorname{rank}(d G)=1$, then there exists a unique (up to scaling) right invariant vector field on $X$ which is everywhere tangent to $f(\Sigma)$.

Proof Suppose first that $d G$ has rank zero at some point $q \in \Sigma$. By [28, equation (3.9) and Corollary 3.21], there exists a two-dimensional subgroup $\Lambda$ of $X$ which has the same mean curvature as $f$ and (constant) left invariant Gauss map of value $G(q) \in \mathbb{S}^{2}$. We are going to prove next that $f(\Sigma)=$ $l_{f(q)}(\Lambda)=f(q) \Lambda$.

Consider the foliation $\mathcal{F}=\left\{l_{a}(\Lambda)=a \Lambda \mid a \in X\right\}$ of left cosets of $\Lambda$. Then, we may write the leaves of $\mathcal{F}$ as the level sets of a Morse function without critical points $h: X \rightarrow \mathbb{R}$, so that $\{h=0\}=f(q) \Lambda$. Also, we can consider local coordinates $\left(x_{1}, x_{2}, x_{3}\right)$ in $X$ around $f(q)$ with $x_{3}:=h$, so that $f(q)=(0,0,0)$ in these coordinates. By taking a small neighborhood $V$ of $f(q)$ in $f(\Sigma)$, we may view $V$ as a graph $x_{3}=\varphi\left(x_{1}, x_{2}\right)$ over a small neighborhood $U$ of the origin in the $\left(x_{1}, x_{2}\right)$-plane.

Arguing by contradiction, assume that $f(\Sigma)$ and $f(q) \Lambda$ do not coincide in a neighborhood of $f(q)$. Then, $\varphi$ is not identically zero around the origin. Since the graphs $x_{3}=\varphi\left(x_{1}, x_{2}\right)$ and $x_{3}=0$ have the same constant mean 
curvature in $X$ (and thus define solutions to the same quasilinear elliptic PDE), then $\varphi-0=\varphi$ satisfies a second order linear homogeneous elliptic equation with smooth coefficients. So, by Bers' theorem [4], we have $\varphi\left(x_{1}, x_{2}\right)=$ $p_{k}\left(x_{1}, x_{2}\right)+o\left(\sqrt{x_{1}^{2}+x_{2}^{2}}\right)^{k}$, where $p_{k}\left(x_{1}, x_{2}\right)$ is a homogeneous polynomial of the form $p_{k}=h_{k} \circ \Phi$, with $h_{k}$ a harmonic homogeneous polynomial of degree $k \geq 2$ in $\mathbb{R}^{2}$, and $\Phi$ a linear transformation of the $\left(x_{1}, x_{2}\right)$-coordinates. In particular, the vector field $D \varphi:=\left(\varphi_{x_{1}}, \varphi_{x_{2}}\right)$ on $U$ has at the origin an isolated zero of negative index.

As $f$ is a pointed limit immersion of $\mathcal{C}$, there exist a sequence $\left\{\widehat{f}_{n}: S_{n} \rightarrow\right.$ $X\}_{n} \subset \mathcal{C}$, compact domains $\Omega_{n} \subset S_{n}$ and points $p_{n} \in \Omega_{n}$ such that the conditions (G1) and (G2) hold. For $n$ large, let $V(n) \subset \widehat{f_{n}}\left(\Omega_{n}\right)$ be subdomains that are expressed as small normal graphs over $V$ and converge to $V$ as $n \rightarrow \infty$. Thus, the $V(n)$ can be seen as graphs $x_{3}=\varphi_{n}\left(x_{1}, x_{2}\right)$ over $U$, making $U$ smaller if necessary. Since the $\varphi_{n}$ converge to $\varphi$, we have that for $n$ large enough, the winding number of each $D \varphi_{n}:=\left(\left(\varphi_{n}\right)_{x_{1}},\left(\varphi_{n}\right)_{x_{2}}\right)$ along a sufficiently small circle around the origin is negative. Thus, by degree theory, for $n$ large enough we have $D \varphi_{n}=(0,0)$ at some point $z_{n} \in U$, what means that the graph $x_{3}=\varphi_{n}\left(x_{1}, x_{2}\right)$ intersects tangentially some leaf $x_{3}=a_{n}$ of $\mathcal{F}$ at $\left(z_{n}, a_{n}\right)$. Note that, by the Transversality Lemma, the sphere $\widehat{f}_{n}\left(S_{n}\right)$ is tangent to the foliation $\mathcal{F}$ at exactly two points; in particular, $\varphi_{n}$ has then a local extremum at $z_{n}$ and no other critical points in $U$ except for, possibly, another local extremum. This contradicts the fact that the winding number of $D \varphi_{n}$ is negative around the origin, by Poincaré-Hopf. Thus, $f(\Sigma)=f(q) \Lambda$, and $d G$ has rank zero everywhere.

Suppose now that the rank of $d G$ is constant one on $\Sigma$. By item 1 of Proposition 4.1, $f(\Sigma)$ is invariant under the flow of a nonzero right invariant vector field $K$ on $X$. If $\widetilde{K}$ is a right invariant vector field on $X$ linearly independent from $K$, and $\widetilde{K}$ is everywhere tangent to $f(\Sigma)$, then the Lie bracket $[K, \widetilde{K}]$ is also everywhere tangent to $f(\Sigma)$. This implies that $K, \widetilde{K}$ generate an integrable two-dimensional subalgebra of the algebra of right invariant vector fields on $X$, whose integral submanifold passing through $e$ is a two-dimensional subgroup $\Lambda$ of $X$. By uniqueness of integral submanifolds of an integrable distribution, we have $f(\Sigma)=\Lambda$, which contradicts that the rank of $d G$ is constant one on $\Sigma$. Hence item 2 of the proposition is proved.

We next prove that if $d G$ has rank one at a some point $q \in \Sigma$, then the rank of $d G$ is one everywhere on $\Sigma$, which together with the previous paragraphs implies the constancy of the rank of $d G$ in any case and finishes the proof of Proposition 5.4. Suppose then that $\operatorname{rank}\left(d G_{q}\right)=1$ at some $q \in \Sigma$. By the argument in Step 1 of the proof of [26, Theorem 4.1], there is a nonzero right invariant vector field $V$ on $X$ that has a contact at $f(q)$ with $f(\Sigma)$ of order at least two. Suppose for the moment that $V$ is everywhere tangent to $f(\Sigma)$. In this case, the differential of $G$ has rank at most one at every point of $\Sigma$ by 
item 1 of Proposition 4.1. But by the previous arguments of this proof, the rank of $d G$ cannot be zero at any point of $\Sigma$, and so, $d G$ has constant rank one.

To finish the proof, we assume that $V$ is not everywhere tangent to $f(\Sigma)$ and we will obtain a contradiction. This contradiction will follow from the existence of two disjoint compact domains in the index-one $H_{n}$-spheres $\widehat{f_{n}}: S_{n} \rightarrow X$ that give rise to the limit immersion $f$ (in the sense of conditions (G1) and (G2) above), such that each of these compact domains is unstable. To create these compact subdomains, we proceed as follows. Consider the Jacobi function $J=\langle V, N\rangle$ on $\Sigma$, where $N$ stands for the unit normal vector field along $f$. By [5, Theorem 2.5], in a small compact neighborhood $E_{q}$ of $q$ in $\Sigma$, the set $J^{-1}(0)$ has the appearance of a set of $k$ embedded arcs $\alpha_{1}, \ldots, \alpha_{k}$ crossing at equal angles at $q$ with very small geodesic curvatures (their geodesic curvatures all vanish at the common point $q$ ), where $k \geq 2$ is the degree of vanishing of $J$ at $q$; see Fig. 2. Let $E(n) \subset \Omega_{n}$ be compact disks that converge to $E_{q}$ as $n \rightarrow \infty$, where $\Omega_{n} \subset S_{n}$ is defined in condition (G1) above. As $S_{n}$ has index one, any Jacobi function on $S_{n}$ is a second eigenfunction of its stability operator. Hence, it follows by Courant's nodal domain theorem that any Jacobi function on $S_{n}$ has at most two nodal domains; see e.g. [10] for a similar argument. Since $S_{n}$ is simply connected, we conclude then that the zero set of the Jacobi function $J_{n}=\left\langle V, N_{n}\right\rangle$ (here $N_{n}$ is the unit normal vector of $\left.\widehat{f_{n}}\right)$ is a regular, connected, analytic Jordan curve that decomposes $S_{n}$ into two nodal domains $D_{1}(n), D_{2}(n)$, each of them diffeomorphic to a disk, and the zero sets $J_{n}^{-1}(0) \cap \Omega_{n}$ converge as $n \rightarrow \infty$ to the zero set $J^{-1}(0)$ of $J$. Then one of the two nodal domains, say $D_{1}(n)$, intersects $E(n)$ in a connected set, see Fig. 2.

Let $U_{1}, U_{2} \subset \Sigma$ be two small compact disjoint geodesic disks of radius $\varepsilon_{0}>0$ centered at points $q_{1} \in \alpha_{1}, q_{2} \in \alpha_{2}$ such that $U_{1} \cup U_{2} \subset E_{q}$ and $\left(U_{1} \cup\right.$ $\left.U_{2}\right) \cap \alpha_{j}=\emptyset$ for all $j \neq 1,2$. Let $q_{1}(n), q_{2}(n) \in J_{n}^{-1}(0) \cap E(n)$ be points in the zero set of $J_{n}$ that converge to $q_{1}, q_{2}$, respectively, and let $U_{1}(n), U_{2}(n)$ be $\varepsilon_{0}$-disks in $E(n)$ centered at the points $q_{1}(n), q_{2}(n)$ that converge to $U_{1}, U_{2}$ as $n \rightarrow \infty$. Choose a compact embedded short $\operatorname{arc} \beta_{n} \subset D_{1}(n)-\left[U_{1}(n) \cup U_{2}(n)\right]$ such that

(H1) $\beta_{n}$ joins points in different components of $E(n) \cap \partial D_{1}(n)$, and $\beta_{n}$ separates $U_{1}(n) \cap D_{1}(n)$ from $U_{2}(n) \cap D_{1}(n)$ in $D_{1}(n)$.

(H2) $\left\{x \in S_{n} \mid d_{S_{n}}\left(x, \beta_{n}\right) \leq \frac{1}{n}\right\}_{n}$ converges to $\{q\}$ as $n \rightarrow \infty$, the length of $\beta_{n}$ is less than $1 / n$ for each $n$ and dist $S_{n}\left(\beta_{n}, U_{1}(n) \cup U_{2}(n)\right)>\delta$ for some small $\delta>0$ independent of $n$, where dist $S_{n}$ denotes intrinsic distance in $S_{n}$.

Note that $\beta_{n}$ decomposes $D_{1}(n)$ into two disk components $D_{1}(n)^{+}, D_{1}(n)^{-}$, each one being disjoint from exactly one of the disks $U_{1}(n), U_{2}(n)$. Without loss of generality, we can assume that $U_{1}(n) \cap D_{1}(n)^{-}=\emptyset$ (and thus, $U_{2}(n) \cap$ 

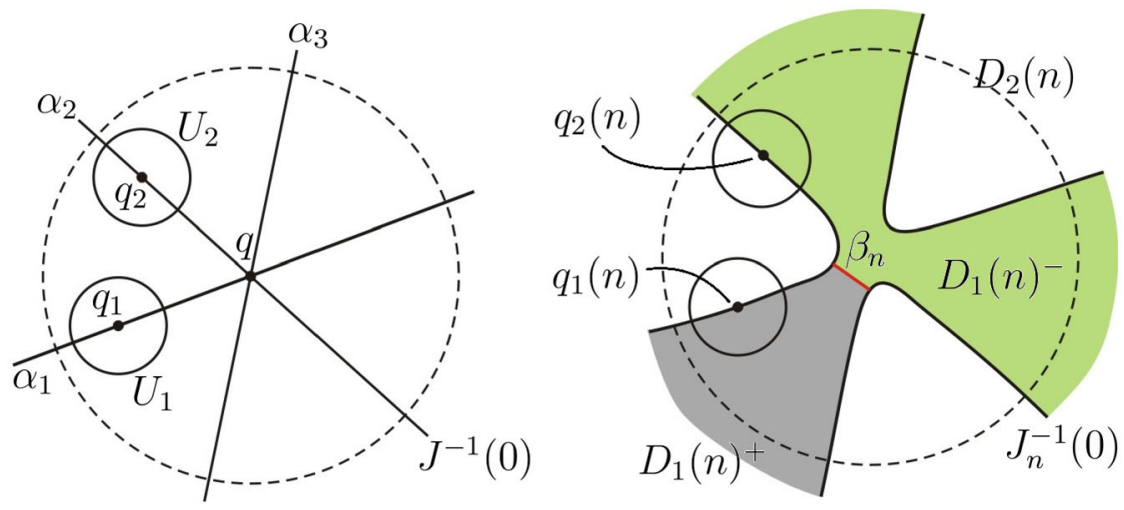

Fig. 2 Left: In a small neighborhood $E_{q}$ (represented as a dotted disk) of the point $q \in \Sigma$, the nodal lines of the Jacobi function $J$ form an equiangular system of (almost geodesic) arcs crossing at $q$. Right: The nodal lines of the related Jacobi functions $J_{n}$ in a (dotted) disk $E(n) \subset S_{n}$ converging to $E_{q} . J_{n}^{-1}(0)$ divides $S_{n}$ into two disk regions $D_{1}(n), D_{2}(n)$, and the short arc $\beta_{n}$ divides $D_{1}(n)$ in $D_{1}(n)^{+}, D_{1}(n)^{-}$. The two small disks centered at $q_{1}(n), q_{2}(n)$ are $U_{1}(n), U_{2}(n)$

$\left.D_{1}(n)^{+}=\emptyset\right)$, see Fig. 2. Let $\phi_{n}: S_{n} \rightarrow[0,1]$ be a piecewise smooth cut-off function with the following properties:

(I1) $\phi_{n}(x)=0$ if $\operatorname{dist}_{S_{n}}\left(x, \beta_{n}\right) \leq \frac{1}{3 n}$.

(I2) $\phi_{n}(x)=1$ if $\operatorname{dist}_{S_{n}}\left(x, \beta_{n}\right) \geq \frac{1}{2 n}$.

(I3) $\left|\nabla \phi_{n}\right| \leq n C$ in $S_{n}$, where $C>0$ is a universal constant.

Note that $\phi_{n} J_{n}$ is a piecewise smooth function that vanishes along $\partial D_{1}(n)^{+}$.

We claim that

$$
\int_{D_{1}(n)^{+}}\left(\left|\nabla\left(\phi_{n} J_{n}\right)\right|^{2}-P_{n} \phi_{n}^{2} J_{n}^{2}\right) \rightarrow 0 \text { as } n \rightarrow \infty
$$

where $\mathcal{L}_{n}=\Delta+P_{n}$ is the stability operator of $S_{n}$, i.e., $P_{n}=\left|\sigma_{n}\right|^{2}+\operatorname{Ric}\left(N_{n}\right)$, $\left|\sigma_{n}\right|$ is the norm of the second fundamental form of $S_{n}$ and $\operatorname{Ric}\left(N_{n}\right)$ denotes the Ricci curvature of $X$ in the direction of the unit normal to $S_{n}$. To prove (5.1), observe that since $\mathcal{L}_{n} J_{n}=0$ on $S_{n}$, then

$$
\left|\nabla J_{n}\right|^{2}-P_{n} J_{n}^{2}=\left|\nabla J_{n}\right|^{2}+J_{n} \Delta J_{n}=\operatorname{div}\left(J_{n} \nabla J_{n}\right) .
$$

Multiplying by $\phi_{n}^{2}$ in (5.2) and integrating on $D_{1}(n)^{+}$we find

$$
\int_{D_{1}(n)^{+}}\left(\phi_{n}^{2}\left|\nabla J_{n}\right|^{2}-P_{n} \phi_{n}^{2} J_{n}^{2}\right)=\int_{D_{1}(n)^{+}} \phi_{n}^{2} \operatorname{div}\left(J_{n} \nabla J_{n}\right) .
$$


On the other hand, $\operatorname{div}\left(\phi_{n}^{2} J_{n} \nabla J_{n}\right)=\frac{1}{2}\left\langle\nabla\left(\phi_{n}^{2}\right), \nabla\left(J_{n}^{2}\right)\right\rangle+\phi_{n}^{2} \operatorname{div}\left(J_{n} \nabla J_{n}\right)$, hence the Divergence Theorem and the fact that $\phi_{n} J_{n}=0$ along $\partial D_{1}(n)^{+}$give that

$$
\int_{D_{1}(n)^{+}} \phi_{n}^{2} \operatorname{div}\left(J_{n} \nabla J_{n}\right)=-\frac{1}{2} \int_{D_{1}(n)^{+}}\left\langle\nabla\left(\phi_{n}^{2}\right), \nabla\left(J_{n}^{2}\right)\right\rangle .
$$

Equations (5.3), (5.4) together with (I1), (I2) and (I3) imply that

$$
\begin{aligned}
& \int_{D_{1}(n)^{+}}\left(\left|\nabla\left(\phi_{n} J_{n}\right)\right|^{2}-P_{n} \phi_{n}^{2} J_{n}^{2}\right) \\
& =\int_{D_{1}(n)^{+}} J_{n}^{2}\left|\nabla \phi_{n}\right|^{2} \\
& ==\int_{\left\{\frac{1}{3 n}<d_{S_{n}}\left(x, \beta_{n}\right)<\frac{1}{2 n}\right\} \cap D_{1}(n)^{+}} J_{n}^{2}(x)\left|\nabla \phi_{n}\right|^{2}(x) \\
& \leq C^{2} n^{2} \cdot \operatorname{Area}\left(\left\{\frac{1}{3 n}<d_{S_{n}}\left(x, \beta_{n}\right)<\frac{1}{2 n}\right\}\right) \cdot \max _{\left\{\frac{1}{3 n}<d_{S_{n}}\left(x, \beta_{n}\right)<\frac{1}{2 n}\right\}}\left(J_{n}^{2}(x)\right) .
\end{aligned}
$$

As $\left\{\left.\widehat{f}_{n}\right|_{\Omega_{n}}\right\}_{n}$ is convergent, we conclude that Area $\left(\left\{\frac{1}{3 n}<d_{S_{n}}\left(x, \beta_{n}\right)<\frac{1}{2 n}\right\}\right)$ can be made smaller than $C_{1} / n^{2}$ for $n$ large, for some $C_{1}>0$ independent of $n$. Therefore, the last right-hand-side is bounded from above by

$$
C^{2} C_{1} \max _{\left\{\frac{1}{3 n}<d_{S_{n}}\left(x, \beta_{n}\right)<\frac{1}{2 n}\right\}}\left(J_{n}^{2}(x)\right) .
$$

which tends to zero as $n \rightarrow \infty$ by the first property in (H2) above, because $J(q)=0$. This proves our claim (5.1).

Next we will define a test function for the stability operator on the domain $D_{1}(n)^{+} \cup U_{1}(n)$.

To start, let us consider for $\varepsilon_{0}>0$ sufficiently small, local coordinates $(x, y)$ around $q_{1}$ in $\Sigma$, so that:

- $(x, y)$ are defined on $\mathbb{D}(\varepsilon)=\left\{(x, y): x^{2}+y^{2} \leq \varepsilon\right\}$ for some $\varepsilon \in\left(0, \varepsilon_{0}\right]$, $q_{1}$ corresponds in these coordinates to $(0,0)$ and $\mathbb{D}(\varepsilon)$ corresponds to a compact neighborhood $V_{1} \subset U_{1}$ of $q_{1}$.

- $\alpha_{1} \cap V_{1}$ corresponds to the $\operatorname{arc} \mathbb{D}(\varepsilon) \cap\{x=0\}$.

- The curves $\{y=$ constant $\}$ are geodesic arcs orthogonal to $\{x=0\} \subset \alpha_{1}$.

By noting the convergence of the disks $U_{1}(n)$ to $U_{1}$ as $n \rightarrow \infty$, it is clear that we can choose coordinates $\left(x_{(n)}, y_{(n)}\right)$ in $S_{n}$ around $q_{1}(n)$ for each $n$ large enough on domains $V_{1}(n)$ converging to $V_{1}$, given by a slight deformation of the coordinates $(x, y)$, and with similar properties. More specifically, for $n \geq n_{0}(\varepsilon)$ large enough there exist local coordinates around $q_{1}(n)$ in $S_{n}$, that will be denoted for simplicity also by $(x, y)$, so that: 

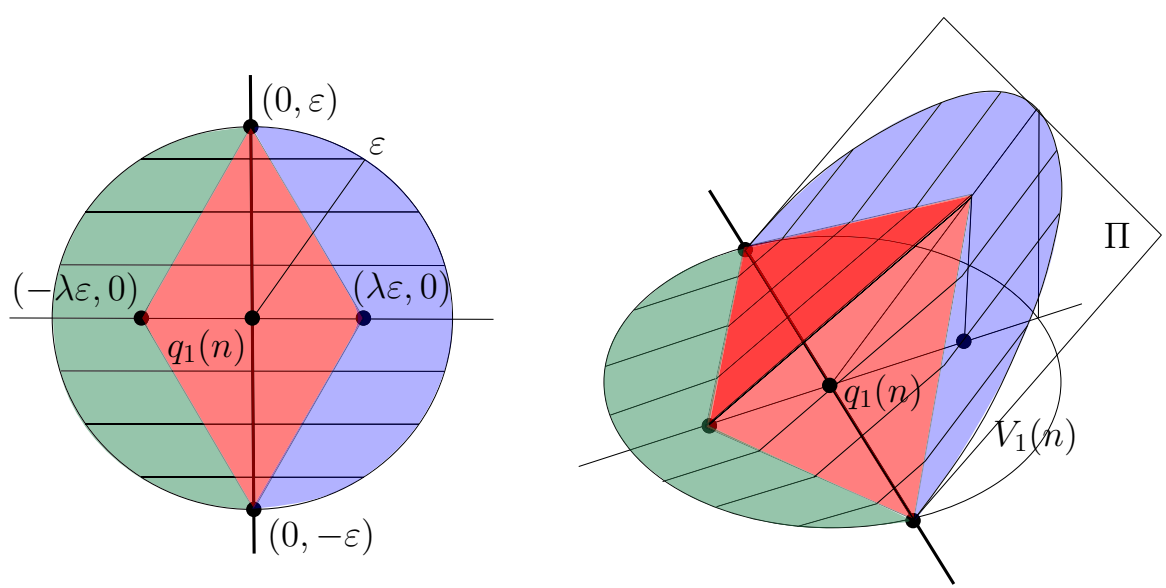

Fig. 3 Left: The compact neighborhood $V_{1}(n) \equiv\left\{x^{2}+y^{2} \leq \varepsilon^{2}\right\}$ of $q_{1}(n)=(0,0)$ intersects $\{x \geq 0\}$ in $D_{1}(n)^{+} \cap V_{1}(n) . V_{1}(n)$ contains the rhombus $R(n)$ (in red color). Right: The function $v_{1}(n)$ is zero in the green region, coincides with $J_{n}$ in the blue region and linearly interpolates these boundary values on $\partial R(n)$ as a function of $x$ in the interior of $R(n)$ (we have represented $J_{n}$ as a linear graph whose slope at $q_{1}(n)$ is that of the tilted plane $\Pi$ ) (color figure online)

- $(x, y)$ are defined on $\mathbb{D}(\varepsilon)=\left\{(x, y): x^{2}+y^{2} \leq \varepsilon\right\}$ for some $\varepsilon \in\left(0, \varepsilon_{0}\right]$, $q_{1}(n)$ corresponds in these coordinates to $(0,0)$ and $\mathbb{D}(\varepsilon)$ corresponds to a compact neighborhood $V_{1}(n) \subset U_{1}(n)$ of $q_{1}(n)$.

- $D_{1}(n)^{+} \cap V_{1}(n)$ corresponds to $\mathbb{D}(\varepsilon) \cap\{x \geq 0\}$.

- The curves $\{y=$ constant $\}$ are geodesic arcs orthogonal to $\{x=0\} \subset$ $\partial D_{1}(n)^{+}$.

Given $\lambda \in(0,1)$, consider the rhombus $R(n)=R(\varepsilon, \lambda, n) \subset \mathbb{D}(\varepsilon)$ that corresponds to the convex hull of the points $( \pm \lambda \varepsilon, 0),(0, \pm \varepsilon)$ in these coordinates, see Fig. 3.

We define a function $v_{1}(n): \mathbb{D}(\varepsilon) \rightarrow \mathbb{R}$ in these local coordinates as a function of $x, y$ as follows:

(J1) $v_{1}(n)=0$ in $\left[D_{2}(n) \cap V_{1}(n)\right]-R(n)$.

(J2) $v_{1}(n)=J_{n}$ in $\left[D_{1}(n)^{+} \cap V_{1}(n)\right]-R(n)$.

(J3) $v_{1}(n)$ linearly interpolates the values of $\left.J_{n}\right|_{D_{1}(n)^{+} \cap \partial R(n)}$ and the value zero on $\partial R(n) \cap D_{2}(n)$ along the geodesics $\{y=$ constant $\}$.

For $n$ large, $v_{1}(n)$ can be extended to a piecewise smooth map on $S_{n}$ (also denoted by $v_{1}(n)$ ), with the following properties for $\lambda, \varepsilon$ sufficiently small:

$(\mathrm{J} 1)^{\prime} v_{1}(n)=0$ in $S_{n}-\left[D_{1}(n)^{+} \cup R(n)\right]$,

$(\mathrm{J} 2)^{\prime} v_{1}(n)=\phi_{n} J_{n}$ in $D_{1}(n)^{+}-R(n)$,

(J3) $\max _{R(n)} v_{1}(n)^{2} \leq \max _{D_{1}(n)^{+} \cap \partial R(n)} J_{n}^{2}=\max _{D_{1}(n)^{+} \cap R(n)} J_{n}^{2}$.

(J4) $\max _{R(n)}\left|\nabla v_{1}(n)\right| \leq \frac{3}{5} \min _{D_{1}(n)^{+} \cap R(n)}\left|\nabla J_{n}\right|$. 
Observe that $(\mathrm{J} 1)^{\prime},(\mathrm{J} 2)^{\prime}$ respectively follow from $(\mathrm{J} 1),(\mathrm{J} 2)$ and the fact that $\phi_{n}=1$ in $U_{1}(n)$ for $n$ large. We explain next why properties $(\mathrm{J} 3)^{\prime}$ and $(\mathrm{J} 4)^{\prime}$ hold for $v_{1}(n)$.

To start, note that, in terms of the $(x, y)$ coordinates in $V_{1}(n)$, the Jacobi function $J_{n}$ satisfies $J_{n}(0, y)=0$ and $\left|\nabla J_{n}\right|(0, y) \neq 0$ for every $y \in[-\varepsilon, \varepsilon]$. Thus, for $\varepsilon$ small enough, $J_{n}$ can be arbitrarily well approximated in $\mathbb{D}(\varepsilon)$ by a linear function of the type $a x$ for some $a \neq 0$. This shows that the equality in $(\mathrm{J} 3)^{\prime}$ holds for $\varepsilon, \lambda$ small enough. The inequality in $(\mathrm{J} 3)^{\prime}$ is immediate from the definition of $v_{1}(n)$.

Similarly, by the previous comments and the definition by interpolation of $v_{1}(n)$ on $R(n)$, we see that $v_{1}(n)$ is arbitrarily well-approximated in $R(n) \subset$ $\mathbb{D}(\varepsilon)$ for $\varepsilon$ small enough by the piecewise linear function $\frac{a}{2}(x-\lambda|y|+\lambda \varepsilon)$, where $a \neq 0$ is the previous constant associated to $J_{n}$. Thus, the (almost constant) norm of the gradient of $v_{1}(n)$ in $R(n) \subset \mathbb{D}(\varepsilon)$ is approximately $\sqrt{1+\lambda^{2}} / 2$ times the (almost constant) norm of the gradient of $J_{n}$ in $\mathbb{D}(\varepsilon)$. By taking $\lambda$ small enough, we obtain condition $(\mathrm{J} 4)^{\prime}$.

Observe that $v_{1}(n)$ vanishes along the boundary of $D_{1}(n)^{+} \cup R(n)$. We will prove that, for $\varepsilon, \lambda$ sufficiently small, there is $n_{0}=n_{0}(\varepsilon, \lambda)$ such that, whenever $n \geq n_{0}$, the following inequality holds:

$$
\int_{D_{1}(n)+\cup R(n)}\left(\left|\nabla v_{1}(n)\right|^{2}-P_{n} v_{1}(n)^{2}\right)<0 .
$$

Assuming (5.5) holds, we conclude that $D_{1}(n)^{+} \cup R(n)$ is a strictly unstable domain in $S_{n}$, and thus, $D_{1}(n)^{+} \cup U_{1}(n)$ is strictly unstable as well. Arguing in a similar way we also conclude that $D_{1}(n)^{-} \cup U_{2}(n)$ is strictly unstable. As $D_{1}(n)^{+} \cup U_{1}(n), D_{1}(n)^{-} \cup U_{2}(n)$ have disjoint interiors, we contradict that the index of $S_{n}$ is one. Hence it only remains to prove (5.5) in order to find the desired contradiction in the case that the differential $d G$ has rank one at some point but the right invariant Killing vector field $V$ is not everywhere tangent to $\Sigma$. We next show (5.5).

Since $v_{1}(n)=\phi_{n} J_{n}$ in $D_{1}(n)^{+}-R(n)$, then

$$
\begin{aligned}
& \int_{D_{1}(n)^{+} \cup R(n)}\left(\left|\nabla v_{1}(n)\right|^{2}-P_{n} v_{1}(n)^{2}\right) \\
& =\int_{D_{1}(n)^{+}-R(n)}\left(\left|\nabla\left(\phi_{n} J_{n}\right)\right|^{2}-P_{n} \phi_{n}^{2} J_{n}^{2}\right) \\
& +\int_{R(n)}\left(\left|\nabla v_{1}(n)\right|^{2}-P_{n} v_{1}(n)^{2}\right) .
\end{aligned}
$$


Using property (I2) above, we split the first integral in the right-hand-side of (5.6) for $n$ large as

$$
\int_{D_{1}(n)^{+}}\left(\left|\nabla\left(\phi_{n} J_{n}\right)\right|^{2}-P_{n} \phi_{n}^{2} J_{n}^{2}\right)-\int_{D_{1}(n)^{+} \cap R(n)}\left(\left|\nabla J_{n}\right|^{2}-P_{n} J_{n}^{2}\right) .
$$

By the previous claim in Eq. (5.1), the first integral in (5.7) tends to zero as $n \rightarrow \infty$. Hence, to prove (5.5) we just need to show that if $\varepsilon, \lambda$ are sufficiently small and $n$ is large enough, then

$$
\begin{aligned}
& \int_{R(n)}\left(\left|\nabla v_{1}(n)\right|^{2}-P_{n} v_{1}(n)^{2}\right)-\int_{D_{1}(n)^{+} \cap R(n)}\left(\left|\nabla J_{n}\right|^{2}-P_{n} J_{n}^{2}\right) \\
& \quad \leq C(\varepsilon)
\end{aligned}
$$

for some constant $C(\varepsilon, \lambda)<0$.

First note that for $\varepsilon, \lambda$ sufficiently small and $n$ large enough (once $\varepsilon, \lambda$ are fixed), we have by (J3)'

$$
\begin{aligned}
& \left|-\int_{R(n)} P_{n} v_{1}(n)^{2}+\int_{D_{1}(n)^{+} \cap R(n)} P_{n} J_{n}^{2}\right| \\
& \quad \leq 2 \max _{R(n)}\left|P_{n}\right| \cdot \max _{D_{1}(n)^{+} \cap R(n)}\left(J_{n}^{2}\right) \cdot \operatorname{Area}[R(n)] \\
& \quad \leq 2 \max _{R(n)}\left|P_{n}\right| \cdot \varepsilon^{2} \max _{D_{1}(n)^{+} \cap R(n)}\left|\nabla J_{n}\right|^{2} \cdot \operatorname{Area}[R(n)] .
\end{aligned}
$$

Observe that the potential $P_{n}$ converges smoothly to the corresponding potential $P=|\sigma|^{2}+\operatorname{Ric}(N)$ for the limit surface $\Sigma$. Therefore, $\max _{R(n)}\left|P_{n}\right|$ can be supposed to be less than some $\mu>0$ independent of $\varepsilon, \lambda$. This implies that

$$
\begin{aligned}
& 2 \max _{R(n)}\left|P_{n}\right| \cdot \varepsilon^{2} \max _{D_{1}(n)^{+} \cap R(n)}\left|\nabla J_{n}\right|^{2} \cdot \operatorname{Area}[R(n)] \\
& \stackrel{(A)}{\leq} 4 \mu \cdot \varepsilon^{2} \min _{D_{1}(n)^{+} \cap R(n)}\left|\nabla J_{n}\right|^{2} \cdot \operatorname{Area}[R(n)],
\end{aligned}
$$

where in $(\mathrm{A})$ we have used that the sequences of numbers

$$
\left\{\max _{D_{1}(n)^{+} \cap V_{1}(n)}\left|\nabla J_{n}\right|\right\}_{n}, \quad\left\{\min _{D_{1}(n)^{+} \cap V_{1}(n)}\left|\nabla J_{n}\right|\right\}_{n}
$$

converge to the same positive limit $\left|(\nabla J)\left(q_{1}\right)\right|$ when we make $\varepsilon>0$ decrease to zero, and take $n$ large enough with respect to each such choice of $\varepsilon$.

On the other hand,

$$
\int_{R(n)}\left|\nabla v_{1}(n)\right|^{2}-\int_{D_{1}(n)^{+} \cap R(n)}\left|\nabla J_{n}\right|^{2}
$$




$$
\begin{aligned}
& \leq \max _{R(n)}\left|\nabla v_{1}(n)\right|^{2} \cdot \operatorname{Area}[R(n)]-\min _{D_{1}(n)^{+} \cap R(n)}\left|\nabla J_{n}\right|^{2} \cdot \operatorname{Area}\left[D_{1}(n)^{+} \cap R(n)\right] \\
& \stackrel{(B)}{\leq} \max _{R(n)}\left|\nabla v_{1}(n)\right|^{2} \cdot \operatorname{Area}[R(n)]-\min _{D_{1}(n)^{+} \cap R(n)}\left|\nabla J_{n}\right|^{2} \cdot \frac{2}{5} \operatorname{Area}[R(n)],
\end{aligned}
$$

where (B) holds for $\varepsilon$ small and $n$ large enough; here, we are using that by taking $\varepsilon$ small enough, the metric space structure on $S_{n}$ induced by $\widehat{f}_{n}$ can be assumed to be arbitrarily close to the flat one of the $(x, y)$-coordinates. By property $(\mathrm{J} 4)^{\prime}$, we obtain from the above inequality for $\varepsilon, \lambda$ small and $n$ large that

$$
\begin{aligned}
& \int_{R(n)}\left|\nabla v_{1}(n)\right|^{2}-\int_{D_{1}(n)^{+} \cap R(n)}\left|\nabla J_{n}\right|^{2} \\
& \leq\left(\frac{9}{25}-\frac{2}{5}\right) \min _{D_{1}(n)^{+} \cap R(n)}\left|\nabla J_{n}\right|^{2} \cdot \operatorname{Area}[R(n)] .
\end{aligned}
$$

Hence, (5.9), (5.10) and (5.11) give that

$$
\begin{aligned}
& \int_{R(n)}\left(\left|\nabla v_{1}(n)\right|^{2}-P_{n} v_{1}(n)^{2}\right)-\int_{D_{1}(n)^{+} \cap R(n)}\left(\left|\nabla J_{n}\right|^{2}-P_{n} J_{n}^{2}\right) \\
& \leq\left(4 \mu \varepsilon^{2}-\frac{1}{25}\right) \min _{D_{1}(n)^{+} \cap R(n)}\left|\nabla J_{n}\right|^{2} \cdot \operatorname{Area}[R(n)],
\end{aligned}
$$

which implies directly that inequality (5.8) holds for $\varepsilon, \lambda$ small enough and $n$ large. It then follows that for $n$ large, there exist two disjoint unstable regions on $S_{n}$ which contradicts that $S_{n}$ has index one. This completes the proof of the proposition.

Definition 5.5 Suppose $f^{\prime}:\left(\Sigma^{\prime}, p^{\prime}\right) \rightarrow(X, e)$ is a pointed limit immersion of $\mathcal{C}$. We define $\Delta\left(f^{\prime}\right)$ as the set of pointed immersions $f:(\Sigma, p) \rightarrow(X, e)$ where $\Sigma$ is a complete, noncompact connected surface, $p \in \Sigma, f(p)=e$ and $f$ is obtained as a limit of $f^{\prime}$ under an (intrinsically) divergent sequence of left translations. In other words, there exist compact domains $\Omega_{n}^{\prime} \subset \Sigma^{\prime}$ and points $q_{n} \in \Omega_{n}^{\prime}$ diverging to infinity in $\Sigma^{\prime}$ such that the sequence of left translated immersions $\left\{\left.\left(l_{f^{\prime}\left(q_{n}\right)^{-1}} \circ f^{\prime}\right)\right|_{\Omega_{n}^{\prime}}\right\}_{n}$ converges on compact sets of $\Sigma$ to $f$ as $n \rightarrow \infty$.

Proposition 5.6 Let $f^{\prime}:\left(\Sigma^{\prime}, p^{\prime}\right) \rightarrow(X, e)$ be a pointed limit immersion of $\mathcal{C}$. Then, the space $\Delta\left(f^{\prime}\right)$ is nonempty and every $[f:(\Sigma, p) \rightarrow(X, e)] \in \Delta\left(f^{\prime}\right)$ satisfies:

(1) $f$ is a limit surface of $\mathcal{C}$, thus of constant mean curvature $h_{0}(X)$.

(2) $f$ is stable.

(3) There exists a nonzero right invariant vector field on $X$ which is everywhere tangent to $f(\Sigma)$. 
(4) $f(\Sigma)$ is topologically an immersed plane or annulus in $X$.

(5) $\Sigma$ is diffeomorphic to a plane or an annulus.

Moreover, if $f(\Sigma)$ is not a two-dimensional subgroup of $X$, then the left invariant Gauss map image $G(\Sigma)$ of $f$ is a regular curve in $\mathbb{S}^{2}$.

Proof The fact that $\Delta\left(f^{\prime}\right)$ is not empty and items 1,2 of the proposition follow directly from the main statement of [26, Corollary 5.4]. Item 3 of the proposition was proved in item 1 of [26, Corollary 5.4], under the extra assumption (K1) below (as in our explanation previous to Proposition 4.1, assumption (K1) was stated in [26, Corollary 5.4] in terms of the $H$-potential, so one must use [28, Corollary 3.21] to reformulate it in the following manner):

(K1) No left translation of a two-dimensional subgroup in $X$ with constant mean curvature $h_{0}(X)$ is tangent to $f(\Sigma)$ at some point.

Therefore, item 3 of the proposition will be proved if we demonstrate that

(K2) If $f(\Sigma)$ is not a two-dimensional subgroup, then property (K1) holds.

We next prove (K2). Arguing by contradiction, suppose that $f(\Sigma)$ is not a two-dimensional subgroup and there exist a two-dimensional subgroup $\Lambda$ of $X$ with mean curvature $h_{0}(X)$, and points $x \in X, y \in f(\Sigma) \cap(x \Lambda)$ such that $f(\Sigma)$ and $x \Lambda=l_{x}(\Lambda)$ are tangent at $y$. Therefore, $f(\Sigma), x \Lambda$ are different surfaces tangent at $y$ with the same unit normal at this point and the same constant mean curvature. Since $f$ is a limit surface of $\mathcal{C}$ by the already proven item 1 of this proposition, we can easily adapt the arguments in the first part of the proof of Proposition 5.4 to deduce that $f(\Sigma)=x \Lambda$, which is impossible since $e \in f(\Sigma)$, and so $f(\Sigma)=\Lambda$, a subgroup. Therefore, property (K2) holds and the proof of item 3 of Proposition 5.6 is complete.

Once item 3 is proved, the proofs of items 4, 5 of the proposition are the same as the proofs of the related items 2, 3 of [26, Corollary 5.4], respectively.

It remains to prove the 'Moreover part' of the proposition, so assume that $f(\Sigma)$ is not a two-dimensional subgroup. By properties (K1), (K2) above, we conclude that there are no two-dimensional subgroups in $X$ with mean curvature $h_{0}(X)$, whose (constant) left invariant Gauss map lies in $G(\Sigma)$. In this setting, the already proven item 3 of this proposition implies that we can apply Proposition 4.1 to deduce that $f(\Sigma)$ is a regular curve. This completes the proof.

The next technical lemma will be used in the proof of Corollary 5.9.

Lemma 5.7 (Unique Limit Surface Lemma). Let $f_{1}:\left(\Sigma_{1}, p\right) \rightarrow(X, e)$ be a pointed limit immersion of $\mathcal{C}$, with associated pointed immersions $\widehat{f_{n}}:\left(S_{n}, p_{n}\right) \leftrightarrow(X, e), S_{n} \in \mathcal{C}$, so that each $H_{n}$-sphere $S_{n}$ contains a compact subdomain $\Omega_{n}^{1}$ with $p_{n} \in \Omega_{n}^{1}$ and $\left.\widehat{f}_{n}\right|_{\Omega_{n}^{1}}$ converges to $f_{1}$ as $n \rightarrow \infty$, 
and Area $\left(\left.\widehat{f}_{n}\right|_{\Omega_{n}^{1}}\right)>n$. Suppose that the rank of the differential $d G^{1}$ of the left invariant Gauss map $G^{1}$ of $f_{1}$ is one. Let $G_{n}$ be the left invariant Gauss map of $\widehat{f}_{n}$ and let $q_{n} \in S_{n}$ be the unique point with $G_{n}\left(q_{n}\right)=v=G^{1}(p)$. Then, after choosing a subsequence, there exist compact domains $\Omega_{n}^{2} \subset S_{n}$ with $q_{n} \in \Omega_{n}^{2}$ such that:

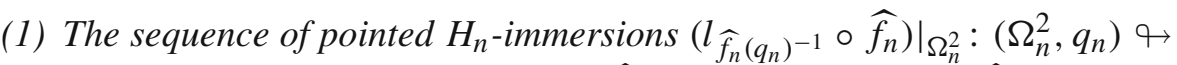
$(X, e)$ obtained by left translating $\left.\widehat{f}_{n}\right|_{\Omega_{n}^{2}}$ by the inverse of $\widehat{f}_{n}\left(q_{n}\right)$ in $X$, converges to a pointed immersion $f_{2}:\left(\Sigma_{2}, q\right) \rightarrow(X, e)$ that is a limit of $\mathcal{C}$, and which has unit normal vector $v$ at $q \in \Sigma_{2}$.

(2) The immersion $f_{2}$ has the same image as $f_{1}$.

Remark 5.8 Lemma 5.7 remains true if we drop the hypothesis that the rank of $d G^{1}$ is one, but we will not need this more general version in this paper.

Proof The existence of the compact subdomains $\Omega_{n}^{2} \subset S_{n}$ (after passing to a subsequence) and item 1 follow from standard arguments in elliptic theory, as we have uniform curvature estimates for the $H_{n}$-immersions $l_{\widehat{f}_{n}\left(q_{n}\right)^{-1}} \circ$ $\widehat{f_{n}}:\left(S_{n}, q_{n}\right) \leftrightarrow(X, e)$ by item 5 of Proposition 3.4. By construction, the $h_{0}(X)$-immersions $f_{1}, f_{2}$ have an oriented contact of order at least one at $e$. By Proposition 5.4, the differentials of the respective Gauss maps $G^{1}$ and $G^{2}$ of $f_{1}$ and $f_{2}$ have constant ranks, which are possibly different. We now distinguish cases depending on the value of $\operatorname{rank}\left(d G^{2}\right)$.

(L1) Suppose $\operatorname{rank}\left(d G^{2}\right)=0$. In this case, item 1 of Proposition 5.4 gives that $f_{2}$ is injective and $f_{2}\left(\Sigma_{2}\right)$ is a two-dimensional subgroup of $X$. Since $f_{1}(\Sigma)$ is not a two-dimensional subgroup (because $\operatorname{rank}\left(d G^{1}\right)=1$ ), and both $f_{1}\left(\Sigma_{1}\right), f_{2}\left(\Sigma_{2}\right)$ are tangent at $e$ with the same constant mean curvature, we can apply the arguments in the first part of the proof of Proposition 5.4 (see also the proof of property (K2) above) to find a contradiction. Therefore, this case cannot occur.

(L2) Suppose $\operatorname{rank}\left(d G^{2}\right)=1$. In this case, $G^{1}\left(\Sigma_{1}\right)$ and $G^{2}\left(\Sigma_{2}\right)$ are analytic immersed curves $\alpha_{1}, \alpha_{2}$ in $\mathbb{S}^{2}$ passing through $v \in \mathbb{S}^{2}$. If $\alpha_{1}, \alpha_{2}$ intersect tangentially at $v$, then by Lemma 4.3 we have $f_{1}\left(\Sigma_{1}\right)=f_{2}\left(\Sigma_{2}\right)$ and so the lemma holds. So, assume next that $\alpha_{1}, \alpha_{2}$ are transverse at $v$. Thus, given $\varepsilon>0$ small enough there is some $n_{0}=n_{0}(\varepsilon)$ such that, if $n \geq n_{0}$, the images through $G_{n}$ of the intrinsic balls $B_{S_{n}}\left(p_{n}, \varepsilon\right), B_{S_{n}}\left(q_{n}, \varepsilon\right)$ centered at $p_{n}$ and $q_{n}$ of radius $\varepsilon$, must intersect in an open nonempty set of $\mathbb{S}^{2}$ near $v$. As $G_{n}$ is a diffeomorphism, $B_{S_{n}}\left(p_{n}, \varepsilon\right) \cap B_{S_{n}}\left(q_{n}, \varepsilon\right) \neq \emptyset$, which implies that $d_{S_{n}}\left(p_{n}, q_{n}\right)<2 \varepsilon$. Therefore, $d_{S_{n}}\left(p_{n}, q_{n}\right) \rightarrow 0$ as $n \rightarrow \infty$, and this shows by item 2 of Remark 5.2 that $f_{1}\left(\Sigma_{1}\right)=f_{2}\left(\Sigma_{2}\right)$. This proves Lemma 5.7 in this case.

(L3) Suppose $\operatorname{rank}\left(d G^{2}\right)=2$. By the Inverse Function Theorem applied to $G^{2}$ around $q$, we can find $\varepsilon>0$ small such that if $B_{\varepsilon}$ denotes the intrinsic 
ball in $\Sigma_{2}$ of radius $\varepsilon$ centered at $q$, then $\left.G^{2}\right|_{B_{\varepsilon}}$ is a diffeomorphism onto its image. Choosing $\varepsilon>0$ small enough, we can assume that for $n$ large, $G^{2}\left(B_{\varepsilon}\right) \subset G_{n}\left(B_{S_{n}}\left(q_{n}, 2 \varepsilon\right)\right)$, where $B_{S_{n}}\left(q_{n}, 2 \varepsilon\right)$ is the intrinsic ball of radius $2 \varepsilon$ around $q_{n}$ in $S_{n}$. As $G_{n}\left(p_{n}\right) \rightarrow v$, we have $G_{n}\left(p_{n}\right) \in$ $G_{n}\left(B_{S_{n}}\left(q_{n}, 2 \varepsilon\right)\right)$, and as $G_{n}$ is a diffeomorphism, this implies that $p_{n} \in$ $B_{S_{n}}\left(q_{n}, 2 \varepsilon\right)$ for $n$ large enough. As $\varepsilon$ is arbitrarily small, we conclude that $d_{S_{n}}\left(p_{n}, q_{n}\right) \rightarrow 0$ as $n \rightarrow \infty$. This implies that the limit surfaces $f_{1}\left(\Sigma_{1}\right), f_{2}\left(\Sigma_{2}\right)$ are the same by item 2 of Remark 5.2 , so in particular $\operatorname{rank}\left(d G^{2}\right)=1$, a contradiction. Therefore, this case does not occur, and this completes the proof of Lemma 5.7.

As a consequence of Lemma 5.7 and the Transversality Lemma (Lemma 5.3), we have the following corollary.

Corollary 5.9 Suppose $f:(\Sigma, p) \rightarrow(X, e) \in \Delta\left(f^{\prime}\right)$, where $f^{\prime}:\left(\Sigma^{\prime}, p^{\prime}\right) \rightarrow$ $(X, e)$ is a limit immersion of $\mathcal{C}$. If $f(\Sigma)$ is tangent at some point to a left or right coset of a two-dimensional subgroup of $X$, then $f(\Sigma)$ is contained in one of the two closed complements of this coset in $X$.

Proof Suppose the image immersed surface $f(\Sigma)$ is tangent to a left or right coset $E$ of some two-dimensional subgroup of $X$ at a point $f(y) \in f(\Sigma) \cap E$, where $y \in \Sigma$. By the discussion just before property (C) in Sect. 4.1, we can assume that $E=f(y) \Lambda$ for some two-dimensional subgroup $\Lambda$ of $X$. After a left translation of $f$ by $f(y)^{-1}$, we may assume that $p=y$, and hence $f(\Sigma)$ is tangent to $\Lambda$ at $e$.

If $f(\Sigma)$ is a two-dimensional subgroup of $X$, then $f(\Sigma)=\Lambda$ and there is nothing to prove. Hence in the sequel we will assume that $f(\Sigma)$ is not a two-dimensional subgroup of $X$. In this case, as $f \in \Delta\left(f^{\prime}\right)$ and $f^{\prime}$ is a limit immersion of $\mathcal{C}$, then Proposition 5.6 gives that $G(\Sigma)$ is a regular curve in $\mathbb{S}^{2}$, where $G: \Sigma \rightarrow \mathbb{S}^{2} \subset T_{e} X$ is the left invariant Gauss map of $f$. In particular, $\operatorname{rank}(d G)=1$.

Applying Lemma 5.7 to $f_{1}=f$, we conclude that $f(\Sigma)$ coincides with the image set $f_{2}\left(\Sigma_{2}\right)$ of the limit $f_{2}:\left(\Sigma_{2}, p_{2}\right) \leftrightarrow(X, e)$ of a sequence of pointed immersions $f_{n}^{2}:\left(\Omega_{n}^{2}, q_{n}\right) \rightarrow(X, e)$, where each $\Omega_{n}^{2}$ is a compact subdomain of an $H_{n}$-sphere $S_{n}$ in $\mathcal{C}$ and such that the left invariant Gauss map of $f_{n}^{2}$ at $q_{n} \in \Omega_{n}^{2}$ is $v=G(p)$. By the Transversality Lemma, the spheres $f_{n}^{2}\left(S_{n}\right)$ all lie on one side of $\Lambda$. Thus, $f(\Sigma)=f_{2}\left(\Sigma_{2}\right)=\lim _{n \rightarrow \infty} f_{n}^{2}\left(\Omega_{n}^{2}\right)$ must lie on one side of $\Lambda$. This completes the proof of the corollary.

Lemma 5.10 Suppose $f:(\Sigma, p) \rightarrow(X, e) \in \Delta\left(f^{\prime}\right)$, where $f^{\prime}:\left(\Sigma^{\prime}, p^{\prime}\right) \rightarrow$ $(X, e)$ is a limit immersion for $\mathcal{C}$. For each $q \in \Sigma$, let $f_{q}=l_{f(q)}^{-1} \circ f:(\Sigma, q) \leftrightarrow$ $(X, e)$ denote the related pointed immersion from $(\Sigma, q)$ to $(X, e)$ obtained 
from $f$ after a change of base point and left translating by $f(q)^{-1}$. Consider the set $\Delta(f)$ of limits of $f$ under a divergent sequence left translations, in the sense of Definition 5.5. Then:

(1) $\Delta(f)$ is a subset of $\Delta\left(f^{\prime}\right)$.

Assume that $\Delta(f)$ contains no elements with constant left invariant Gauss map. Then:

(2) The image $\gamma_{f}=G(\Sigma)$ of the left invariant Gauss map $G: \Sigma \rightarrow \mathbb{S}^{2} \subset$ $T_{e} X$ of $f$ is a complete embedded regular curve in $\mathbb{S}^{2}$. Moreover, its closure $\overline{\gamma_{f}} \subset \mathbb{S}^{2}$ admits the structure of a lamination of $\mathbb{S}^{2}$, whose leaves correspond to the Gauss map images $\gamma_{\hat{f}}$ of elements $\widehat{f} \in \Delta(f)$. In particular, if $\widehat{f} \in \Delta(f)$, then $\Delta(\widehat{f}) \subset \Delta(f)$ and so, $\overline{\gamma_{\widehat{f}}}$ is a sublamination of $\overline{\gamma_{f}}$.

(3) There is a uniform upper bound on the absolute geodesic curvature of all the leaves of $\overline{\gamma_{f}}$.

(4) There exists $\widehat{f} \in \Delta(f)$ such that $\overline{\gamma_{\widehat{f}}}$ contains no proper sublaminations. Furthermore, one of the following two possibilities holds:

(a) If $\gamma_{\widehat{f}}$ is a closed curve, then the lamination $\overline{\gamma_{\widehat{f}}}$ contains a single leaf.

(b) If $\gamma_{\widehat{f}}$ is not a simple closed curve, then the lamination $\overline{\gamma_{\widehat{f}}}$ has uncountably many leaves and $\gamma_{\hat{f}}$ has the following recurrency property: given any compact arc I of $\gamma_{\hat{f}}$, there exists a sequence of intrinsically divergent, pairwise disjoint arcs in $\gamma_{\hat{f}}$ that converge to I in the $C^{1}$-topology.

Proof Item 1 follows from a standard diagonal argument.

In the sequel, we will assume that $\Delta(f)$ contains no elements with constant left invariant Gauss map. In particular, $f(\Sigma)$ is not a two-dimensional subgroup of $X$.

We next prove item 2. By Proposition 5.6, there exists a nonzero, right invariant vector field on $X$ which is everywhere tangent to $f(\Sigma)$ and the Gauss map image $\gamma_{f}$ is a regular curve in $\mathbb{S}^{2}$. Next we show that the curve $\gamma_{f}$ is complete, or equivalently, it has no end points in $\mathbb{S}^{2}$. If such an end point $x \in \mathbb{S}^{2}$ of $\gamma_{f}$ exists, then we can consider a divergent sequence $q_{n} \in \Sigma$ and compact geodesic disks $D_{n} \subset \Sigma$ around $q_{n}$ of fixed radius such that $G\left(D_{n}\right) \rightarrow\{x\}$. After passing to a subsequence, there exist compact subdomains $\Omega_{n} \subset \Sigma$ with $q_{n} \in \Omega_{n}$ such that the restrictions $f_{q_{n}} \mid \Omega_{n}$ converge to an element $\widetilde{f} \in \Delta(f)$ (see the first paragraph in the proof of Lemma 5.7 for a similar argument). By construction, the Gauss map of $\widetilde{f}$ is constantly equal to $x$ around $e$. So, by analyticity, $\widetilde{f}$ has constant left invariant Gauss map, which contradicts our hypothesis. Therefore, $\gamma_{f}$ is complete.

By the arguments in the proof of Lemma 4.3, $\gamma_{f}$ has no tangential selfintersections. Transversal self-intersections of $\gamma_{f}$ can also be ruled out by using a straightforward modification of the arguments in case (L2) of the proof of 
Lemma 5.7. Therefore, the curve $\gamma_{f}$ is embedded. Also, note that the surface $f(\Sigma)$ can be viewed locally as the graph of a solution to a quasilinear elliptic PDE, and recall that $f$ has uniformly bounded second fundamental form. By standard elliptic estimates, this provides a priori $C^{3}$ estimates for $f(\Sigma)$, and this shows in particular that $\gamma_{f}$ has bounded geometry. These properties for $\gamma_{f}$ imply that its closure $\overline{\gamma_{f}}$ has the structure of a lamination of $\mathbb{S}^{2}$, all whose leaves have bounded geometry as well. Hence item 3 of the lemma is proved.

If $\widehat{f} \in \Delta(f)$, then the arguments above apply to $\widehat{f}$ to give that the Gauss map image $\gamma_{\widehat{f}}$ of $\widehat{f}$ is a complete embedded regular curve in $\mathbb{S}^{2}$. By definition, there exists a sequence $\left\{q_{n}^{\prime}\right\}_{n} \subset \Sigma$ such that the immersions $f_{q_{n}^{\prime}}$ converge smoothly to $\widehat{f}$, and thus the corresponding Gauss map images $\gamma_{f_{q_{n}^{\prime}}}$ converge to $\gamma_{\hat{f}}$ in $\mathbb{S}^{2}$. But clearly $\gamma_{q_{n}^{\prime}}=\gamma_{f}$ as sets, from where we deduce that the leaves of the lamination $\overline{\gamma_{f}}$ correspond to the Gauss map images of elements of $\Delta(f)$. This proves item 2 of the lemma.

We finish by proving item 4 . Consider the set $\mathcal{S}$ of sublaminations of the lamination $\overline{\gamma_{f}}$, which is partially ordered by the inclusion. We want to apply Zorn's lemma to $\mathcal{S}$ in order to find a minimal element in $\mathcal{S}$, i.e., a sublamination of $\overline{\gamma_{f}}$ with no proper sublaminations. To do this, we must check that every totally ordered subset $\mathcal{S}_{1}$ of $\mathcal{S}$ has a lower bound. This is clear provided that the intersection of all sublaminations in $\mathcal{S}_{1}$ is nonempty. Since $\mathcal{S}_{1}$ is a collection of closed sets of the compact topological space $\mathbb{S}^{2}$ and $\mathcal{S}_{1}$ clearly satisfies the finite intersection property, then the intersection of all sublaminations in $\mathcal{S}_{1}$ is nonempty. By Zorn's lemma, there exists a sublamination $\mathcal{L}$ of $\overline{\gamma_{f}}$ with no proper sublaminations. Take a leaf $\Gamma$ of $\mathcal{L}$. As $\Gamma$ is a leaf of $\overline{\gamma_{f}}$, then item 2 implies that there exists an immersion $\widehat{f} \in \Delta(f)$ whose Gauss map image $\gamma_{\widehat{f}}$ is equal to $\Gamma$. Therefore, $\overline{\gamma_{\widehat{f}}}$ is a sublamination of $\mathcal{L}$, and by minimality of $\mathcal{L}$ in $\mathcal{S}$ implies that $\overline{\gamma_{\widehat{f}}}=\mathcal{L}$. This proves the first sentence of item 4 of the lemma.

In order to prove items $4 \mathrm{a}$ and $4 \mathrm{~b}$, it is worth adapting some known facts about laminations to our setting. Take a sublamination $\overline{\gamma_{\widehat{f}}}$ of $\overline{\gamma_{f}}$ with no proper sublaminations, corresponding to an element $\widehat{f} \in \Delta(f)$. A point $x \in \overline{\gamma_{\widehat{f}}}$ is called a limit point of $\overline{\gamma \widehat{f}}$ if $x$ is the limit in $\mathbb{S}^{2}$ of an intrinsically divergent sequence $\left\{x_{n}\right\}_{n} \subset \gamma_{\widehat{f}}$. If $x \in \overline{\gamma_{\widehat{f}}}$ is a limit point of $\overline{\gamma_{\widehat{f}}}$, then the leaf component $L$ of the lamination $\overline{\gamma_{\widehat{f}}}$ that contains $x$ consists entirely of limit points of $\overline{\gamma_{\widehat{f}}}$ (and $L$ is called a limit leaf). The $\operatorname{set} \operatorname{Lim}\left(\gamma_{\widehat{f}}\right)$ of limit points of $\overline{\gamma_{\widehat{f}}}$ is a (closed) sublamination of $\overline{\gamma_{\widehat{f}}}$, possibly empty.

If $\gamma_{\widehat{f}}$ is a closed curve, then clearly $\operatorname{Lim}\left(\gamma_{\widehat{f}}\right)$ is empty and so, $\overline{\gamma_{\widehat{f}}}$ consists of the single leaf $\gamma_{\hat{f}}$, which is item $4 \mathrm{a}$ of the lemma. Next suppose that $\gamma_{\widehat{f}}$ is not a simple closed curve. Thus, $\operatorname{Lim}\left(\gamma_{\widehat{f}}\right) \neq \emptyset$. Since $\overline{\gamma_{\widehat{f}}}$ contains no proper sublaminations, then $\operatorname{Lim}\left(\gamma_{\widehat{f}}\right)=\overline{\gamma_{\widehat{f}}}$. We next show that $\overline{\gamma_{\widehat{f}}}$ contains an uncountable number of leaves. Consider a small compact arc $\alpha \subset \mathbb{S}^{2}$ cutting $\overline{\gamma_{\widehat{f}}}$ transversally. Since complete one-manifolds are second countable in 
their intrinsic topology (each leaf of the lamination $\overline{\gamma_{\hat{f}}}$ has this property), to prove that $\overline{\gamma_{\widehat{f}}}$ has an uncountable number of leaves, as a lamination has a local product structure it suffices to prove that $W=\alpha \cap \overline{\gamma_{\hat{f}}}$ is uncountable. We can consider $W$ to be a complete metric space (note that $\alpha$ is a compact arc in $\mathbb{S}^{2}$ with its usual topology, hence $\alpha$ has a natural structure of a complete metric space, and $W$ is a closed subset of $\alpha$ ). $W$ has no isolated points, since $\operatorname{Lim}\left(\gamma_{\widehat{f}}\right)=\overline{\gamma_{\hat{f}}}$. In this setting, the uncountability of $W$ is a consequence of the following well-known elementary application of the Baire category theorem: any complete metric space without isolated points is uncountable.

Finally, the recurrency property in item $4 \mathrm{~b}$ of the lemma follows easily from the fact that $\gamma_{\widehat{f}} \subset \overline{\gamma_{\widehat{f}}}=\operatorname{Lim}\left(\gamma_{\widehat{f}}\right)$. Now Lemma 5.10 is proved.

For the remainder of this section we will use the notation introduced in Lemma 5.10, and denote by $f:(\Sigma, p) \rightarrow(X, e)$ an element of $\Delta\left(f^{\prime}\right)$ such that $\Delta(f)$ contains no elements with constant left invariant Gauss map. In particular, $f(\Sigma)$ is not a two-dimensional subgroup of $X$.

By Proposition 5.6, $\gamma_{f}$ is a regular curve in $\mathbb{S}^{2}$ and thus, the right invariant vector field $K_{\Sigma}$ given by item 2 of Proposition 5.4 is unique up to scaling. Once we pick $K_{\Sigma}$ and an orientation on $X$, the curve $\gamma_{f} \subset \mathbb{S}^{2}$ has a natural orientation as follows.

Definition 5.11 Suppose that $q \in \Sigma$ and $N(q) \in T_{f(q)} X$ is the unit normal vector to $\Sigma$ at $q$. Consider a short $\operatorname{arc} \beta$ in $\Sigma$ transverse to the integral curves of the vector field $\widetilde{K}_{\Sigma}$ induced by $K_{\Sigma}$ on $\Sigma$, parameterized so that $\beta(0)=q$ and $\left\{d f_{q}\left(\beta^{\prime}(0)\right), K_{\Sigma}(f(q)), N(q)\right\}$ is a positively oriented basis for $T_{f(q)} X$. Now, the orientation on $\gamma_{f}$ is defined to be the one given by $G \circ \beta$ where $G$ is the Gauss map of $f$.

Lemma 5.12 Assume that $\gamma_{f}$ is not a simple closed curve in $\mathbb{S}^{2}$. Then, there exists a compact interval $\sigma \subset \gamma_{f}$ with $G(p) \in \sigma$ (here $G$ is the left invariant Gauss map of $f$ and $p$ is the base point of $f$ ) and there exists a sequence of pairwise disjoint arcs $\sigma_{n} \subset \gamma_{f}$ that are small normal graphs over $\sigma$, which converge to $\sigma$ in the $C^{1}$-topology and, as graphs oriented by $\gamma_{f}$, satisfy that their induced orientations are opposite of the orientation of $\sigma$, see Fig. 4.

Proof By item 4b of Lemma 5.10, given a small arc $\sigma \subset \gamma_{f}$ containing $G(p)$, there exists a sequence of pairwise disjoint intervals $\sigma_{n} \subset \gamma_{f}$ which converge $C^{1}$ in $\mathbb{S}^{2}$ to $\sigma$. After extracting a subsequence, we can assume that all the $\operatorname{arcs} \sigma_{n}$ lie on the same side of $\sigma$; more specifically, we may assume that there exists a one-sided neighborhood $U$ of $\sigma$ diffeomorphic to a square $[0,1] \times[0,1]$, so that $\sigma$ corresponds to $\{0\} \times[0,1]$ and for each $n \in \mathbb{N}, \sigma_{n}$ corresponds to $\{1 / n\} \times[0,1]$ under this diffeomorphism. Also note that the lamination structure on the closure of $\gamma_{f}$ ensures that we can take $U$ so that 
Fig. 4 If $\gamma_{f}$ is not a simple closed curve, then it contains compact arcs $\sigma_{n}$ converging to a compact arc $\sigma$, with the orientations of the $\sigma_{n}$ being opposite to the one of $\sigma$

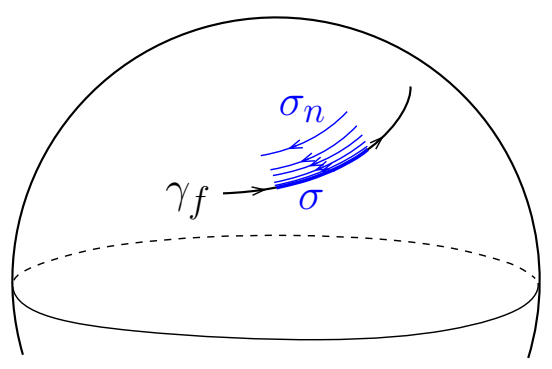

Fig. 5 The future of $I^{\prime}$ in $\gamma_{f}$ must enter $D$ by crossing $U$ pointing down, a contradiction

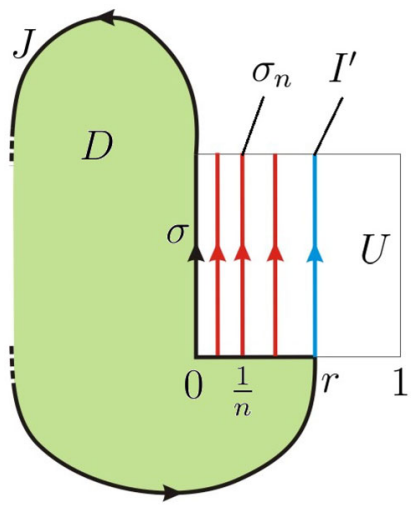

every component of $\gamma_{f} \cap U$ is a small normal graph over $\sigma$. Therefore, in the model of $U$ as $[0,1] \times[0,1], \gamma_{f} \cap U$ can be represented as $A \times[0,1]$ where $A \subset[0,1]$ is a compact infinite subset, $1 \in A$ and 0 is an accumulation point of $A$.

Without loss of generality, we can also assume that the orientation on $\sigma$ induced by the one of $\gamma_{f}$ is pointing upwards in this model of $\sigma$ as $\{0\} \times[0,1]$. If Lemma 5.12 fails, then we have that after choosing $U$ small enough, every component of $\gamma_{f} \cap U \equiv A \times[0,1]$ is oriented by pointing upwards. We can assume that $\gamma_{f}$ is parameterized by the real line $\mathbb{R}$, and its orientation is the induced one by this parameterization. Then, each of the segments $\sigma, \sigma_{n}$ corresponds respectively to a closed interval $I, I_{n} \subset \mathbb{R}$ by this parameterization, and these intervals form a pairwise disjoint collection. Each point or closed interval $J$ in $\mathbb{R}$ defines a "future" (the component of $\mathbb{R}-J$ that limits to $+\infty$ ) and a "past" (the component of $\mathbb{R}-J$ that limits to $-\infty$ ), and these definitions can be translated to $\gamma_{f}$ via the parameterization. After passing to a subsequence, we can assume that one of the two following possibilities holds:

(M1) $I_{n}$ is contained in the future of $I$, for all $n \in \mathbb{N}$.

(M2) $I_{n}$ is contained in the past of $I$, for all $n \in \mathbb{N}$.

If case (M1) holds, then consider the end point $t_{1}=\max (I)$ and the first $t_{2}>t_{1}$ whose image by the parameterization lies in the closed topological 
square $U \subset \mathbb{S}^{2}$; the image of the interval $\left(t_{1}, t_{2}\right) \subset \mathbb{R}$ is an open Jordan arc $J$ in $\mathbb{S}^{2}-U$ with endpoints in $\partial U$ corresponding to the points $(0,1)$ and $(r, 0)$ for a certain $r \in(0,1]$, in the model of $U$ as $[0,1] \times[0,1]$, see Fig. 5. By definition, $J \cap U=\emptyset$ and $(r, 0)$ lies in the boundary of an interval component $I^{\prime}$ of $\gamma_{f} \cap U$, which in our square model is represented by $\{r\} \times[0,1]$. Then, $\alpha:=\sigma \cup J \cup([0, r] \times\{0\})$ defines a Jordan curve in $\mathbb{S}^{2}$. Let $D \subset \mathbb{S}^{2}$ be the topological disk bounded by $\alpha$ whose interior is disjoint from the square $U$. Consider the embedded open $\operatorname{arc}\left(I^{\prime}\right)^{+}$of $\gamma_{f}$ given by the future of $I^{\prime}$. Note that $\left(I^{\prime}\right)^{+}$must eventually intersect the interior of $D$ (to see this, observe that for $n$ sufficiently large, the segment $\sigma_{n} \equiv\{1 / n\} \times[0,1]$ lies in $\left(I^{\prime}\right)^{+}$and intersects $\partial D$ transversely at its initial point). Since $\gamma_{f}$ has no self-intersections, we deduce that $\left(I^{\prime}\right)^{+}$must cross $\partial D$ along the arc $(0, r) \times\{0\}$. Moreover, at the points of $\left(I^{\prime}\right)^{+} \cap \partial D$ along the arc $(0, r) \times\{0\}$ where $\left(I^{\prime}\right)^{+}$enters $D$, the induced orientation of $\left(I^{\prime}\right)^{+}$points down, which contradicts our assumption that every component of $\gamma_{f} \cap U \equiv A \times[0,1]$ is oriented by pointing up. If possibility (M2) above occurs, then a similar argument leads to a contradiction. This finishes the proof of Lemma 5.12.

Lemma 5.13 Suppose that $\gamma_{f}$ is not a closed curve. Let $\sigma, \sigma_{n} \subset \gamma_{f}, n \in \mathbb{N}$, be the arcs given by Lemma 5.12, and assume that $G(p) \in \sigma$ (here $p$ is the base point of $f$ and $G$ is its left invariant Gauss map). Let $D, D_{n} \subset \Sigma$ be given by $D:=G^{-1}(\sigma), D_{n}:=G^{-1}\left(\sigma_{n}\right)$. Take points $p_{n} \in D_{n}$ so that $\left\{G\left(p_{n}\right)\right\}_{n}$ converges to $G(p)$. Then, there exist diffeomorphisms $\Phi_{n}: D \rightarrow D_{n}$ such that

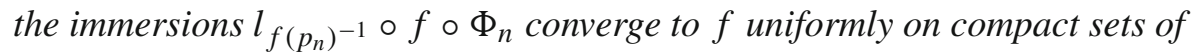
$D$.

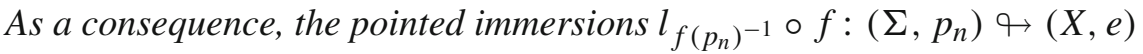
converge uniformly on compact sets to $f:(\Sigma, p) \rightarrow(X, e)$.

Proof As $\gamma_{f}$ is not a closed curve, $\Sigma$ cannot be diffeomorphic to an annulus, and so by Proposition 5.6 it is simply connected. Thus, by the arguments described after Proposition 4.1 and the uniformization theorem, we can parameterize $f$ as a conformal immersion $f: \mathcal{U} \subset \mathbb{C} \rightarrow X$ where $\mathcal{U}$ is either $\mathbb{C}$ or a vertical strip $\left\{s+i t \mid a_{1}<s<a_{2}\right\}$, and the left invariant Gauss map $G: \mathcal{U} \rightarrow \mathbb{S}^{2}$ of $f$ depends solely on $s$. From now on, we will identify $\Sigma$ with $\mathcal{U}$ and assume that the imaginary part of the base point $p \in \mathcal{U}$ of $f$ is zero. We will also consider the stereographically projected Gauss map $g:=\pi \circ G: \mathcal{U} \rightarrow \overline{\mathbb{C}}$, where $\pi: \mathbb{S}^{2} \rightarrow \overline{\mathbb{C}}$ is the stereographic projection from the South pole of $\mathbb{S}^{2}$ defined in terms of a previously chosen left invariant orthonormal frame $\left\{E_{1}, E_{2}, E_{3}\right\}$ of $X$ (see the paragraph before [26, Definition 3.2]). By item 2 of Lemma 5.10, $s \mapsto g(s)$ is a complete embedded regular curve in $\overline{\mathbb{C}}$. Moreover, after choosing an adequate left invariant frame $\left\{E_{1}, E_{2}, E_{3}\right\}$, [26, Theorem 3.7] implies that $g(s)$ is a solution of a second 
order analytic autonomous ODE, see (4.2). In particular, $g(s)$ satisfies property (D), introduced just before Lemma 4.3.

Write $\sigma=g\left(\left[b_{1}, b_{2}\right]\right)$, and $\sigma_{n}=g\left(I_{n}\right)$, where $\left[b_{1}, b_{2}\right]$ and each $I_{n}$ are compact arcs in $\left(a_{1}, a_{2}\right)$; note that $p$ can be then identified with some point in $\left[b_{1}, b_{2}\right]$. Let $\Phi_{n}(z)=\delta_{n} z+\mu_{n}$, where $\delta_{n}, \mu_{n} \in \mathbb{R}$ satisfy that $\Phi_{n}\left(\left[b_{1}, b_{2}\right]\right)=$ $I_{n}$. Then, we can consider the sequence of maps $g_{n}:=g \circ \Phi_{n}:\left[b_{1}, b_{2}\right] \rightarrow \overline{\mathbb{C}}$. It follows from property (D) above and from the $C^{1}$ convergence of the arcs $\sigma_{n}$ to $\sigma$ given by Lemma 5.12 that the following properties hold:

(N1) Each $g_{n}$ is a solution of the ODE (4.2). In fact, $g_{n}$ is the Gauss map of $f \circ \Phi_{n}$ restricted to $\left[b_{1}, b_{2}\right] \times \mathbb{R} \subset \mathbb{C}$.

(N2) $\left\{g_{n}\right\}_{n} \rightarrow g$ uniformly on $\left[b_{1}, b_{2}\right]$ in the $C^{1}$-topology.

Let $t_{n} \in\left[b_{1}, b_{2}\right]$ such that $g_{n}\left(t_{n}\right)$ is the value of the left invariant Gauss map of $f$ at $p_{n}$. By hypothesis on the points $p_{n}$, we have that $\left\{t_{n}\right\}_{n} \rightarrow p \in\left[b_{1}, b_{2}\right]$.

We next translate property (N2) above into the desired convergence of $l_{f\left(p_{n}\right)^{-1}} \circ f \circ \Phi_{n}$ to $f$. To do this, we observe that given $z \in \mathcal{U}$, we have

$$
f_{z}(z)=\sum_{i=1}^{3} A_{i}(z)\left(E_{i}\right)_{f(z)},
$$

where $A_{1}, A_{2}, A_{3}: \mathcal{U} \rightarrow \mathbb{C}$ are given in terms of $g$ by [26, equation (3.4)], and $\left\{E_{1}, E_{2}, E_{3}\right\}$ is the orthonormal basis of left invariant vector fields on $X$ that we prescribed previously. Note that the immersion $f \circ \Phi_{n}$ satisfies the same complex linear differential equation (5.12) with respect to $g_{n}$. Using properties (N1), (N2) above and the smooth dependence of the solution of an ODE with respect to initial conditions, we deduce that $l_{f\left(p_{n}\right)^{-1}} \circ f \circ \Phi_{n}$ converges uniformly on compact sets of $\left[b_{1}, b_{2}\right] \times \mathbb{R}$ to $f$.

Finally we prove the last sentence in the statement of Lemma 5.13. Now

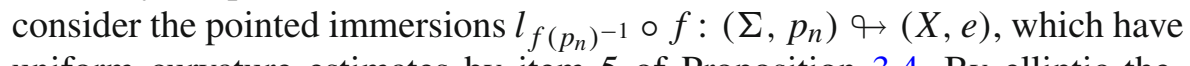
uniform curvature estimates by item 5 of Proposition 3.4. By elliptic theory, these immersions converge (up to subsequence) to some other complete pointed immersion of constant mean curvature. Now, the uniform convergence property that we obtained in the previous paragraph, and the unique continuation principle for surfaces of constant mean curvature (see e.g., Aronszajn [3]) concludes the proof of Lemma 5.13.

\section{The existence of periodic invariant limit surfaces}

In this section we will apply the results of Sect. 5 to prove Theorem 6.1 below, as well as its consequence, Corollary 6.7. In later sections we will also quote some of the steps in the proof of Theorem 6.1. 
We will use the following notation throughout this section. $X$ is a metric Lie group diffeomorphic to $\mathbb{R}^{3} ; h_{0}(X) \geq 0, \mathcal{C}$ are respectively the number and the component of the space $\mathcal{M}_{X}^{1}$ of index-one spheres with constant mean curvature in $X$ that appear in Proposition 3.4. $f:(\Sigma, p) \rightarrow(X, e)$ is a pointed limit immersion of $\mathcal{C}$ produced by Proposition 5.6; in particular, $f$ is stable with constant mean curvature $h_{0}(X)$, and there exists a nonzero, right invariant vector field $K_{\Sigma}$ on $X$ which is everywhere tangent to $f(\Sigma) . \Gamma_{\Sigma} \subset X$ will denote the 1-parameter subgroup of $X$ generated by $K_{\Sigma}$.

Observe that if the space $\Delta(f)$ defined in Lemma 5.10 contains an element $f_{1}:\left(\Sigma_{1}, p_{1}\right) \leftrightarrow(X, e)$ with constant left invariant Gauss map (equivalently, $f_{1}\left(\Sigma_{1}\right)$ is a two-dimensional subgroup of $\left.X\right)$, then $f_{1}$ is injective and $f_{1}\left(\Sigma_{1}\right)$ is an entire Killing graph in $X$ with respect to any nonzero right invariant vector field in $X$ transverse to $f_{1}\left(\Sigma_{1}\right)$. In particular, Theorem 1.5 holds trivially in this case (note that by item 1 of Proposition 5.6, $f_{1}$ is a limit surface of $\mathcal{C}$, and so it has the convergence properties in the statement of Theorem 1.5). Therefore, in the sequel we will assume that no element in $\Delta(f)$ has constant left invariant Gauss map; in particular, $f(\Sigma)$ is not a two-dimensional subgroup of $X$.

By Proposition 5.6, $\Sigma$ is either simply connected (with $f(\Sigma)$ being a plane or an annulus) or $\Sigma$ is diffeomorphic to an annulus (so $f(\Sigma)$ is an annulus as well). By Lemma 5.10, the left invariant Gauss map image $\gamma_{f}=G(\Sigma)$ of $f$ is an embedded regular curve in $\mathbb{S}^{2} \subset T_{e} X$, and we can choose $f$ so that the closure $\overline{\gamma_{f}}$ is a lamination of $\mathbb{S}^{2}$ with no proper sublaminations.

Theorem 6.1 In the above situation, $\gamma_{f}$ is a closed curve in $\mathbb{S}^{2}$.

Proof As $\gamma_{f}$ is not a point, then we can consider on $\gamma_{f}$ the orientation given by Definition 5.11. We will divide the proof of the theorem into three assertions for the sake of clarity.

Assertion 6.2 $\gamma_{f}$ is a closed curve if one of the following two conditions hold:

(1) $X$ is isomorphic to $\mathbb{R}^{2} \rtimes_{A} \mathbb{R}$ for some matrix $A \in \mathcal{M}_{2}(\mathbb{R})$ and $K_{\Sigma} \notin$ $\operatorname{Span}\left\{F_{1}, F_{2}\right\}$ where $F_{1}, F_{2}$ are given by $E q$. (4.5).

(2) $X$ is isomorphic to $\widetilde{\mathrm{SL}}(2, \mathbb{R})$ and $K_{\Sigma}$ is either elliptic or parabolic.

Proof of Assertion 6.2 Arguing by contradiction, suppose that $\gamma_{f}$ is not a simple closed curve. Let $\sigma$ and $\left\{\sigma_{n}\right\}_{n}$ be the arcs given in Lemma 5.12, where $q:=G(p)$ lies in $\sigma$. Let $p_{n}$ be points of $\Sigma$ such that the sequence $G\left(p_{n}\right) \in \sigma_{n}$ converges to $q$ as $n \rightarrow \infty$. By Lemma 5.13, the pointed immer-

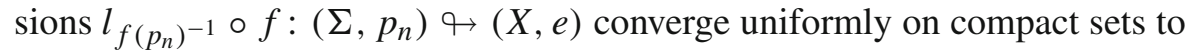
$f:(\Sigma, p) \rightarrow(X, e)$. As $K_{\Sigma}$ is everywhere tangent to $f(\Sigma)$, then the nonzero

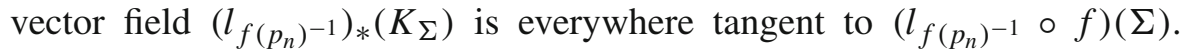

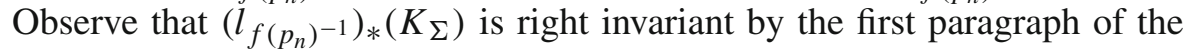


proof of Lemma 4.10. After normalizing, we deduce that the sequence of right invariant vector fields

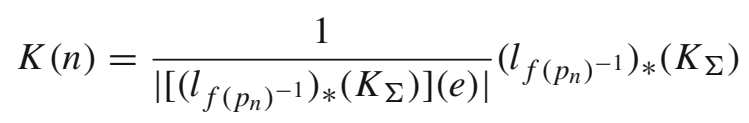

converges to a nonzero right invariant vector field $K_{1}$ on $X$ which is everywhere tangent to $f(\Sigma)$. Since $f(\Sigma)$ is not a two-dimensional subgroup of $X$, then $K_{1}$ must be a (constant) nonzero multiple of $K_{\Sigma}$. As the orientations of the $\operatorname{arcs} \sigma_{n}$ are all opposite to the orientation of the arc $\sigma$, by definition of the orientation of $\gamma_{f}$, we deduce that $K_{1}$ is a negative multiple of $K_{\Sigma}$.

We now check that the last property cannot happen under the hypotheses of Assertion 6.2. First suppose that we are in case 1 of this assertion. Since $K_{\Sigma} \notin \operatorname{Span}\left\{F_{1}, F_{2}\right\}$, then we may assume that

$$
K_{\Sigma}=F_{3}+c_{1} \partial_{x}+c_{2} \partial_{y}
$$

for some constants $c_{1}, c_{2} \in \mathbb{R}$; here $F_{3}$ is given by (4.5). Plugging (4.5) into this last equation we get $K_{\Sigma}=\partial_{z}+h_{1}(x, y) \partial_{x}+h_{2}(x, y) \partial_{y}$ for some functions $h_{1}, h_{2}$. Since $\partial_{z}$ is left invariant and $\left(l_{a}\right)_{*}$ preserves $\operatorname{Span}\left\{\partial_{x}, \partial_{y}\right\}$ for every $a \in X$, then we conclude that $K(n)$ cannot converge to a negative multiple of $K_{\Sigma}$, which is the desired contradiction if case 1 holds.

In case 2 , we can use similar arguments to find a contradiction, using the last sentence of Lemma 4.10. This finishes the proof of Assertion 6.2.

Assertion 6.3 If $X=\mathbb{R}^{2} \rtimes_{A} \mathbb{R}$ and $K_{\Sigma} \in \operatorname{Span}\left\{F_{1}, F_{2}\right\}$, then $\gamma_{f}$ is a closed curve. Moreover, if $\gamma_{f}$ does not pass through the North or South pole, then $f(\Sigma)$ is an entire Killing graph with respect to any horizontal right invariant vector field linearly independent from $K_{\Sigma}$.

Proof of Assertion 6.3 As $K_{\Sigma} \in \operatorname{Span}\left\{F_{1}, F_{2}\right\}$, Eq. (4.5) implies that $K_{\Sigma}$ is a linear combination $\lambda \partial_{x}+\mu \partial_{y}$ for some $\lambda, \mu \in \mathbb{R}$. Since $f(\Sigma)$ is foliated by integral curves of $K_{\Sigma}$, then $f(\Sigma)$ is ruled by straight lines in the direction of $\lambda \partial_{x}+\mu \partial_{y}$. There are two cases to consider:

(O1) The angles between the immersed surface $f(\Sigma)$ and the planes $\mathbb{R}^{2} \rtimes_{A}\{z\}$, $z \in \mathbb{R}$, are bounded away from zero. Equivalently, $\overline{\gamma_{f}}$ does not contain the North or South poles of $\mathbb{S}^{2}$.

(O2) There exists a sequence $\left\{q_{n}\right\}_{n} \subset \Sigma$ such that the angle between $f(\Sigma)$ and $\mathbb{R}^{2} \rtimes_{A}\left\{z\left(f\left(q_{n}\right)\right)\right\}$ at $f\left(q_{n}\right)$ tends to zero as $n \rightarrow \infty$. Equivalently, the North pole or the South pole in $\mathbb{S}^{2}$ is in $\overline{\gamma_{f}}$.

Suppose case (O1) holds. Note that the length of the pullback $\nabla(z \circ f)$ by $f$ of the tangential component of $\partial_{z}$ to $\Sigma$ is bounded away from zero. Without loss of generality, we can assume that $K_{\Sigma}=\partial_{x}$ (this is just a linear change 
of the coordinates $x, y$, which does not affect to our hypotheses). Hence, the image through $f$ of the integral curve of $\nabla(z \circ f)$ passing through the base point $p$ is a curve in $\mathbb{R}^{2} \rtimes_{A} \mathbb{R}$ that passes through the origin and intersects every horizontal plane $\mathbb{R}^{2} \rtimes_{A}\{t\}$ at a single point $Q(t)=(x(t), y(t), t)$. Furthermore, the intersection of $f(\Sigma)$ with $\mathbb{R}^{2} \rtimes_{A}\{t\}$ is the straight line passing through $Q(t)$ in the direction of $\partial_{x}$. Hence, $f(\Sigma)$ is an entire Killing graph with respect to any horizontal right invariant vector field $V=a \partial_{x}+b \partial_{y}$ linearly independent from $\partial_{x}$.

We now prove that $\gamma_{f}$ is a simple closed curve by using arguments that are similar to the ones in the proof of the first item of Assertion 6.2. Consider the cross product $K_{\Sigma} \times N$, which defines a nowhere zero tangent vector field to $f(\Sigma)$ (here $N$ is the unit normal of $f$ ). Thus, the tangent vector field $W$ to $\Sigma$ given by pulling back $K_{\Sigma} \times N$ through $f$ is never tangent to the foliation $\left\{\mathbb{R}^{2} \rtimes_{A}\{z\} \mid z \in \mathbb{R}\right\}$. By connectedness of $\Sigma$, the function $\langle W, \nabla(z \circ f)\rangle$ has constant (nonzero) sign on $\Sigma$. Observe that this sign does not change when we compose $f$ with a left translation in $X$ and do the same process with the translated surface. Assume $\gamma_{f}$ is not a simple closed curve and consider for each $n \in \mathbb{N}$ the right invariant vector field $K(n)$ given by (6.1), which in this case lies in $\operatorname{Span}\left\{\partial_{x}, \partial_{y}\right\}$ (because $\left(l_{a}\right)_{*}$ preserves $\operatorname{Span}\left\{\partial_{x}, \partial_{y}\right\}$ for every $a \in X)$. As before, the $K(n)$ converge to a negative multiple of $K_{\Sigma}$, and so the vector fields $W_{n}$ obtained by pulling back $K(n) \times N_{n}$ through $l_{f\left(p_{n}\right)^{-1} \circ f}$ converge to a negative multiple of $W$ (here, $N_{n}$ denotes the unit normal of

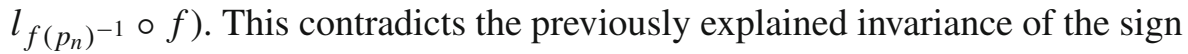
of the function $\langle W, \nabla(z \circ f)\rangle$ under left translations. Hence, $\gamma_{f}$ is a simple closed curve, what proves Assertion 6.3 if case (O1) holds.

In order to analyze case $(\mathrm{O} 2)$, we will prove the following general property to be used later on.

Claim 6.4 (Slab Property in $\mathbb{R}^{2} \rtimes_{A} \mathbb{R}$ ). Suppose $X=\mathbb{R}^{2} \rtimes_{A} \mathbb{R}$ and $K_{\Sigma} \in$ $\operatorname{Span}\left\{F_{1}, F_{2}\right\}$. Let $\widetilde{K}_{\Sigma}=f^{*}\left(K_{\Sigma}\right)$ be the Killing field on $\Sigma$ induced from the right invariant vector field $K_{\Sigma}$. If there exists a sequence $\left\{q_{n}\right\}_{n} \subset \Sigma$ such that the angle between $f(\Sigma)$ and $\mathbb{R}^{2} \rtimes_{A}\left\{z\left(f\left(q_{n}\right)\right)\right\}$ at $f\left(q_{n}\right)$ tends to zero as $n \rightarrow \infty$ (i.e., case (O2) holds), then:

(1) $f(\Sigma)$ is contained in a smallest horizontal slab $\mathbb{R}^{2} \rtimes_{A}[a, b]$ with $a \leq$ $0 \leq b, a \neq b$, and $f(\Sigma)$ is tangent to both $\mathbb{R}^{2} \rtimes_{A}\{a\}$ and $\mathbb{R}^{2} \rtimes_{A}\{b\}$.

(2) If $q \in \Sigma$ satisfies that $f(\Sigma)$ is tangent to $\mathbb{R}^{2} \rtimes_{A}\{z(f(q))\}$, then $z(f(q)) \in$ $\{a, b\}$.

(3) If $\Sigma$ is simply connected, then the points $q_{n}$ can be taken to lie on distinct integral curves of $\widetilde{K}_{\Sigma}$ such that

$$
z\left(f\left(q_{n}\right)\right)= \begin{cases}a & \text { if } n \text { is odd } \\ b & \text { if } n \text { is even } .\end{cases}
$$


(4) $\gamma_{f}$ is a simple closed curve that passes through the North and South poles of $\mathbb{S}^{2}$.

Proof of Claim 6.4 By the uniform curvature estimates in item 5 of Proposition 3.4, after passing to a subsequence, the sequence of left translated pointed

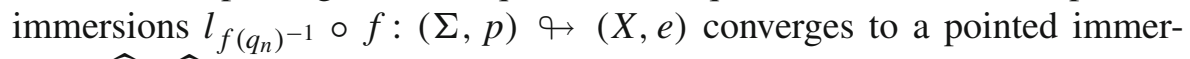
sion $[\widehat{f}:(\widehat{\Sigma}, \widehat{p}) \leftrightarrow(X, e)] \in \Delta(f)$. Since the angle between $f(\Sigma)$ and $\mathbb{R}^{2} \rtimes_{A}\left\{z\left(f\left(q_{n}\right)\right)\right\}$ at $f\left(q_{n}\right)$ is not bounded away from zero, then $\widehat{f}(\widehat{\Sigma})$ is tangent to $\mathbb{R}^{2} \rtimes_{A}\{0\}$ at $e=(0,0,0)$. By Corollary 5.9, $\widehat{f}(\widehat{\Sigma})$ is contained in $\mathbb{R}^{2} \rtimes_{A}(-\infty, 0]$ or $\mathbb{R}^{2} \rtimes_{A}[0, \infty)$. From now on, we will assume that $\widehat{f}(\widehat{\Sigma}) \subset \mathbb{R}^{2} \rtimes_{A}[0, \infty)$ (this does not affect the arguments that follow).

Note that $\widehat{f}$ is invariant under a nonzero right invariant vector field $K_{\widehat{\Sigma}}$, which is the limit as $n \rightarrow \infty$ of the right invariant vector fields $K(n)$ given by Eq. (6.1) after replacing $p_{n}$ by $q_{n}$; in particular, both $K(n)$ and $K_{\widehat{\Sigma}}$ lie in $\operatorname{Span}\left\{\partial_{x}, \partial_{y}\right\}$. Without loss of generality, we can assume that $K_{\widehat{\Sigma}}=\partial_{x}$.

Therefore, $\widehat{f}(\widehat{\Sigma})$ is ruled by straight lines in the direction of $\partial_{x}$. If $\widehat{f}(\widehat{\Sigma})=$ $\mathbb{R}^{2} \rtimes_{A}\{0\}$, then $\widehat{f}$ has constant left invariant Gauss map, which contradicts our assumption stated before Theorem 6.1. Since $\widehat{f}(\widehat{\Sigma})$ is analytic and $\widehat{f}(\widehat{\Sigma}) \neq \mathbb{R}^{2} \rtimes_{A}\{0\}$, then there exists $\varepsilon>0$ such that if $B_{\widehat{\Sigma}}(\widehat{p}, \varepsilon)$ denotes the intrinsic closed disk in $\widehat{\Sigma}$ centered at the base point $\widehat{p}$ with radius $\varepsilon$, then $D=\widehat{f}\left(B_{\widehat{\Sigma}}(\widehat{p}, \varepsilon)\right)$ is a small graphical disk over its vertical projection to $\mathbb{R}^{2} \rtimes_{A}\{0\}, D$ is foliated by line segments in the direction of $\partial_{x}$, exactly one of these line segments lies in the $x$-axis, and all the other ones lie in $\mathbb{R}^{2} \rtimes_{A}(0, \infty)$.

As $l_{f\left(q_{n}\right)^{-1}} \circ f$ converges to $\widehat{f}$, then the sequence of disks $D_{n}=\left(l_{f\left(q_{n}\right)^{-1}} \circ\right.$ $f)\left(B_{\Sigma}\left(q_{n}, \varepsilon\right)\right)$ converges smoothly to $D$, where $B_{\Sigma}\left(q_{n}, \varepsilon\right)$ stands for the intrinsic closed disk in $\Sigma$ centered at $q_{n}$ with radius $\varepsilon$. Consider the integral curves of $K(n)$ (which are horizontal straight lines not necessarily parallel to the $x$-axis) which intersect $D_{n}$. For each $n$, this set of parallel lines forms a smooth surface

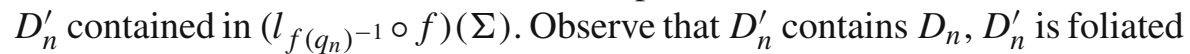
by complete parallel horizontal lines, $\partial D_{n}^{\prime}$ consists of two horizontal lines, and the $D_{n}^{\prime}$ converge smoothly on compact sets as $n \rightarrow \infty$ to the ruled surface $D^{\prime} \subset \widehat{f}(\widehat{\Sigma})$ consisting of the integral curves of $\partial_{x}$ that intersect $D$; note that $D^{\prime}$ contains the $x$-axis, $D^{\prime}$ is foliated by complete lines parallel to the $x$-axis, and its boundary $\partial D^{\prime}$ consists of two lines that both lie in $\mathbb{R}^{2} \rtimes_{A}(0, \infty)$, i.e., above the lowest straight line of $D^{\prime}$. In particular, for $n$ large, the boundary straight lines of $D_{n}^{\prime}$ lie strictly above the lowest straight line in $D_{n}^{\prime}$. Therefore, along this lowest straight line in $D_{n}^{\prime}$, the tangent plane to this surface is horizontal. Hence, $f(\Sigma)$ is also tangent to some horizontal plane and, by Corollary 5.9, we see that $f(\Sigma)$ lies above this plane.

After possibly replacing $f$ by a left translation of it and changing the base point $p$, we may assume that $f(\Sigma)$ is tangent to the plane $P_{0}=\mathbb{R}^{2} \rtimes_{A}\{0\}$ at $f(p)=(0,0,0)$ and it lies above $P_{0}$. 
By analyticity of $f$, we can choose an open strip $\mathcal{S} \subset \Sigma$ containing $p$ and such that:

- $\mathcal{S}$ is invariant under the flow of $\widetilde{K}_{\Sigma}$.

- $\nabla(z \circ f)$ only vanishes in $\mathcal{S}$ along the integral curve $\beta_{1}$ of $\widetilde{K}_{\Sigma}$ that passes through $p$.

- $z \circ f$ attains the same value at the two boundary components of $\mathcal{S}$.

Let $C_{1} \subset \Sigma$ be one of the (at most two) components of $\Sigma-\mathcal{S}$, and let $P^{\prime}$ be the horizontal plane that contains $f\left(\partial C_{1}\right)$. We claim that the angle that $f\left(C_{1}\right)$ makes with the foliation by horizontal planes is not bounded away from zero: otherwise there exists a sequence of points $r_{n} \in \Sigma$ such that $\left|(z \circ f)\left(r_{n}\right)\right| \rightarrow \infty$ as $n \rightarrow \infty$. After left translating $f$ by $f\left(r_{n}\right)^{-1}$ and extracting a subsequence, we find a new limit $f^{\prime} \in \Delta(f)$ such that its image makes angles bounded away from zero with the foliation of horizontal planes $\left\{\mathbb{R}^{2} \rtimes_{A}\{t\} \mid t \in \mathbb{R}\right\}$. Since $\gamma_{f}$ is a leaf of the lamination $\overline{\gamma_{f}}$ and $\overline{\gamma_{f}}=\overline{\gamma_{f^{\prime}}}$ (recall that we were assuming that $\overline{\gamma_{f}}$ has no proper sublaminations), then item 2 of Lemma 5.10 implies that $f$ is also a limit of $f^{\prime}$ under left translations. This clearly contradicts that $f(\Sigma)$ is tangent to $P_{0}$. Hence, our claim is proved.

By repeating the above arguments, there is an integral curve $\beta_{2} \subset C_{1}$ of $\widetilde{K}_{\Sigma}$ such that $\nabla(z \circ f)$ vanishes along $\beta_{2}$ and being the closest such integral curve to $\beta_{1}$ in $C_{1}$; let $b:=(z \circ f)\left(\beta_{2}\right)>0$. As before, Corollary 5.9 implies that $f(\Sigma)$ is contained on one side of the plane $P_{b}=\mathbb{R}^{2} \rtimes_{A}\{b\}$; by connectedness of $f(\Sigma)$, this side must be $\mathbb{R}^{2} \rtimes_{A}(-\infty, b]$, since $f(\Sigma)$ intersects $\mathbb{R}^{2} \rtimes_{A}\{0\}$. This implies that the immersed surface $f(\Sigma)$ is contained in the slab between the planes $P_{0}$ and $P_{b}$; also, the strip $Y_{1} \subset \Sigma$ between $\beta_{1}$ and $\beta_{2}$ has an embedded image contained in that slab (because $z \circ f$ has no critical points in the interior of $Y_{1}$ ). Repeating these arguments in the domain $C_{2}:=C_{1}-Y_{1}$, one finds an integral curve $\beta_{3}$ of $\widetilde{K}_{\Sigma}$ in $C_{2}$ closest to $\beta_{2}$ where $z \circ f$ has a critical value; call $c:=(z \circ f)\left(\beta_{3}\right)$. Note that $0 \leq c<b$, since $f(\Sigma)$ lies between $P_{0}$ and $P_{b}$ and is not equal to $P_{b}$. But also, $f(\Sigma)$ lies on one side of the corresponding horizontal plane $P_{c}$. Again by connectedness of $f(\Sigma)$, and since $f(\Sigma)$ intersects both $P_{0}$ and $P_{b}$, we must have $c=0$. Continuing inductively with this argument, in the case that $\Sigma$ is simply connected, one produces the desired sequence of points $\left\{q_{n}\right\}_{n \in \mathbb{N}}$, where $q_{n} \in \Gamma_{n}$ and for $n$ odd (resp. even) one has the related integral curves $\beta_{n}$ contained in $\mathbb{R}^{2} \rtimes_{A}\{0\}$ (resp. $\mathbb{R}^{2} \rtimes_{A}\{b\}$ ). The remainder of the statements listed in Claim 6.4 follow immediately from this discussion.

Finally, Assertion 6.3 follows from the Slab Property (Claim 6.4) and our previous discussion of case $(\mathrm{O} 1)$.

In the last step of the proof of Theorem 6.1 we will study the case in which $X$ is isomorphic to $\widetilde{\mathrm{SL}}(2, \mathbb{R})$ and $K_{\Sigma}$ is hyperbolic, or equivalently, the 1parameter subgroup $\Gamma_{\Sigma}$ of $\widetilde{\mathrm{SL}}(2, \mathbb{R})$ generated by $K_{\Sigma}$ is hyperbolic. Recall 
from Sect. 4.3 that the image of $\Gamma_{\Sigma}$ through the projection $\Pi: \widetilde{\mathrm{SL}}(2, \mathbb{R}) \rightarrow \mathbb{H}^{2}$ defined by (4.10) is an arc of constant geodesic curvature (possibly zero) in the standard metric of $\mathbb{H}^{2}$, that passes through the origin of the Poincaré disk and its two extrema $\theta_{1}, \theta_{2} \in \partial_{\infty} \mathbb{H}^{2}$ determine the two two-dimensional subgroups $\mathbb{H}_{\theta_{1}}^{2}, \mathbb{H}_{\theta_{2}}^{2}$ of $\widetilde{\mathrm{SL}}(2, \mathbb{R})$ that contain $\Gamma_{\Sigma}$. We will also use in the next statement the pair of antipodal, simple closed curves $\Upsilon \cup(-\Upsilon) \subset \mathbb{S}^{2}$ defined in Lemma 4.11.

Assertion 6.5 If $X$ is isomorphic to $\widetilde{\mathrm{SL}}(2, \mathbb{R})$ and $K_{\Sigma}$ is hyperbolic, then $\gamma_{f}$ is a simple closed curve. Moreover, if $\gamma_{f}$ does not intersect $\Upsilon \cup(-\Upsilon)$, and $\mathbb{H}$ is either $\mathbb{H}_{\theta_{1}}^{2}$ or $\mathbb{H}_{\theta_{2}}^{2}$ (where $K_{\Sigma}$ is tangent to both $\mathbb{H}_{\theta_{1}}^{2}$ and $\mathbb{H}_{\theta_{2}}^{2}$ ), then $f(\Sigma)$ is an entire Killing graph with respect to the parabolic right invariant vector field which is tangent at e to $\mathbb{H}$.

Proof of Assertion 6.5 We denote by $\Gamma_{3}$ the 1-parameter elliptic subgroup of $X$ defined by $\Gamma_{3}^{\prime}(0)=\left(E_{3}\right)_{e}$ (see Eq. 4.7)). Let $\mathbb{H}$ be one of the subgroups $\mathbb{H}_{\theta_{1}}^{2}$ or $\mathbb{H}_{\theta_{2}}^{2}$. Let $\mathcal{F}=\left\{r_{t}(\mathbb{H})=\mathbb{H} t \mid t \in \widetilde{\mathrm{SL}}(2, \mathbb{R})\right\}$ be the foliation of $X$ whose leaves are the right cosets of $\mathbb{H}$. Since $\Gamma_{3}$ intersects $\mathbb{H}$ only at the identity element, it is easily seen that every element of $X$ lies in a unique right coset of $\mathbb{H}$ of the form $\mathbb{H} t$, for some $t \in \Gamma_{3}$.

Consider the oriented distance function $\widetilde{d}: X \rightarrow \mathbb{R}$ to the closed set $\mathbb{H}$, which is positive on $\mathbb{H} t$, for $t>0$, where we have naturally parameterized $\Gamma_{3}$ by $\mathbb{R}$. As each right coset of a codimension one connected subgroup of a metric Lie group is at a constant distance from the subgroup (see [28, Lemma 3.9]) and $\Gamma_{3}$ is isomorphic to $\mathbb{R}$, then the level sets of $\widetilde{d}$ are the leaves of $\mathcal{F}$. Hence, we have an induced function $d: \mathcal{F} \rightarrow \mathbb{R}$ so that the absolute value of $d$ at a given element $\mathbb{H} t$ of $\mathcal{F}$ is the constant distance from $\mathbb{H}$ to $\mathbb{H} t$; thus $\mathcal{F}$ can be parameterized by $\Gamma_{3}$, i.e., $\mathcal{F}=\left\{\mathbb{H} t \mid t \in \Gamma_{3}\right\}$, and we may assume that $\left\langle\nabla \tilde{d}, E_{3}\right\rangle>0$, where here $\nabla$ stands for gradient in $X$.

The approach to prove Assertion 6.5 is to follow, when possible, the arguments in the proof of Assertion 6.3 by exchanging the former foliation $\left\{\mathbb{R}^{2} \rtimes_{A}\{t\} \mid t \in \mathbb{R}\right\}$ by the current one $\mathcal{F}$. Notice that the ambient function $z$ in Assertion 6.3 is now replaced by the oriented distance function $\widetilde{d}: X \rightarrow \mathbb{R}$. For the proof, it is also important to take into account the following list of elementary observations:

(P1) Each of the right cosets $\mathbb{H} t$ with $t \in \mathbb{R} \equiv \Gamma_{3}$ can be written as $\mathbb{H} t=$ $t\left(t^{-1} \mathbb{H} t\right)$, therefore $\mathbb{H} t$ is a left coset of a conjugate two-dimensional subgroup of $\mathbb{H}$; see Sect. 4.1. If $t \in \Gamma_{3}$ lies in the center of $X$ (which is isomorphic to $\mathbb{Z}$ ), then $\mathbb{H} t=t \mathbb{H}$. In this sense, $\mathcal{F}$ is a periodic foliation invariant under the left action of $\mathbb{Z}=\operatorname{center}(X) \subset \Gamma_{3}$.

(P2) Limits of $f$ after left translations by the inverses of points on the image surface $f(\Sigma)$ give rise to limit surfaces $\widehat{f}(\widehat{\Sigma})$ that are invariant under some other hyperbolic right invariant vector field and such that $\gamma_{\widehat{f}}$ is a 
leaf of $\overline{\gamma_{f}}$. Otherwise, Lemma 4.10 implies that $\widehat{f}(\widehat{\Sigma})$ would be tangent to a right invariant parabolic vector field (because the limit of a normalized sequence of hyperbolic right invariant vector fields is either hyperbolic or parabolic). As the set of parabolic vector fields normalized to have length 1 at $e$ is compact, then every element in $\Delta(\widehat{f})$ is tangent to a parabolic right invariant vector field. As $\gamma_{f}$ is a leaf of the lamination $\overline{\gamma_{f}}=\overline{\gamma_{\hat{f}}}$ (here we are using $\overline{\gamma_{f}}$ has no proper sublaminations), then $f$ is itself a limit of $\widehat{f}$, i.e., $f \in \Delta(\widehat{f})$, and so $f$ is tangent to a right invariant parabolic vector field. This is a contradiction since $K_{\Sigma}$ is hyperbolic and $f(\Sigma)$ is not a two-dimensional subgroup of $X$.

(P3) Given $a \in X$, the family $a \mathcal{F}=\left\{a L=l_{a}(L) \mid L \in \mathcal{F}\right\}$ is a foliation of $X$ by the right cosets of some conjugate subgroup of $\mathbb{H}$.

(P4) As the foliation $\mathcal{F}$ is periodic in the sense of item (P1), then for every sequence $\left\{a_{n}\right\}_{n} \subset X$, the sequence of foliations $\left\{a_{n} \mathcal{F}\right\}_{n \in \mathbb{N}}$ defined as in (P3) has a convergent subsequence to another foliation of $X$ by the right cosets of some conjugate subgroup of $\mathbb{H}$.

(P5) Let $\Gamma^{P}$ be the unique 1-parameter parabolic subgroup contained in $\mathbb{H}$. As $\Gamma_{\Sigma} \mathbb{H}=\mathbb{H}$ and $\Gamma^{P} \mathbb{H}=\mathbb{H}$, we deduce that the left actions of $\Gamma_{\Sigma}, \Gamma^{P}$ on $X$ leave invariant each of the right cosets of $\mathbb{H}$. In particular, $K_{\Sigma}$ and the parabolic right invariant vector field $P$ on $X$ generated by $\Gamma^{P}$ are everywhere tangent to each of the leaves of $\mathcal{F}$.

(P6) Given a leaf $\mathbb{H} t$ of $\mathcal{F}$, each integral curve of $K_{\Sigma}$ restricted to $\mathbb{H} t$ intersects each integral curve of $P$ restricted to $\mathbb{H} t$ in exactly one point.

To prove Assertion 6.5, let us start by assuming that the Gaussian image $\gamma_{f}$ is at a positive distance from the set $\Upsilon \cup(-\Upsilon)$. In particular, the angle between $f(\Sigma)$ and the leaves of $\mathcal{F}$ is bounded away from zero. Using observations (P5) and (P6) above, it follows from simple modifications of the arguments in the proof of case (O1) of Assertion 6.3 that in these conditions, $f(\Sigma)$ is an entire Killing graph with respect to $P$, and that $\gamma_{f}$ is closed. Details are left to the reader.

Claim 6.6 (Slab Property in $\widetilde{\mathrm{SL}}(2, \mathbb{R})$ ). Suppose $X=\widetilde{\mathrm{SL}}(2, \mathbb{R})$ and $K_{\Sigma}$ is hyperbolic. Let $\widetilde{K}_{\Sigma}=f^{*}\left(K_{\Sigma}\right)$ be the Killing field on $\Sigma$ induced by the right invariant vector field $K_{\Sigma}$. If there exists a sequence $\left\{q_{n}\right\}_{n} \subset \Sigma$ such that the angle between $f(\Sigma)$ and the leaf of $\mathcal{F}$ passing through $f\left(q_{n}\right)$ tends to zero as $n \rightarrow \infty$, then:

(1) $f(\Sigma)$ is contained in a smallest topological slab bounded by two different leaves $L_{a}, L_{b}$ of $\mathcal{F}$, and $f(\Sigma)$ is tangent to both leaves.

(2) If $f(\Sigma)$ is tangent to a leaf $L$ of $\mathcal{F}$, then $L=L_{a}$ or $L=L_{b}$.

(3) $\gamma_{f}$ is a simple closed curve that intersects both $\Upsilon$ and $-\Upsilon$.

Proof of Claim 6.6 Consider for each $n \in \mathbb{N}$ the left translated pointed immersion $f_{n}:=l_{f\left(q_{n}\right)^{-1}} \circ f:\left(\Sigma, q_{n}\right) \leftrightarrow(X, e)$, which is everywhere tangent to 
the hyperbolic right invariant vector field $K_{n}:=\left(l_{f\left(q_{n}\right)^{-1}}\right)_{*}\left(K_{\Sigma}\right)$; see observation (P2). Note that $K_{n}$ is everywhere tangent to the foliation by right cosets $\mathcal{F}_{n}:=\left\{\mathbb{H}_{n} t \mid t \in \mathbb{R}\right\}$, where $\mathbb{H}_{n}$ is the two-dimensional subgroup of $X$ given by $\mathbb{H}_{n}=f\left(q_{n}\right)^{-1} \mathbb{H} f\left(q_{n}\right)$. After extracting a subsequence, the $f_{n}$ converge on compact sets to a pointed limit immersion $\widehat{f}:(\widehat{\Sigma}, \widehat{p}) \leftrightarrow(X, e)$ and by observation (P4), $\mathcal{F}_{n}$ converges to some foliation $\widehat{\mathcal{F}}$ of $X$ by the right cosets of some two-dimensional subgroup $\widehat{\mathbb{H}}$. Observe that by hypothesis, the angle that $f_{n}(\Sigma)$ makes with $\mathcal{F}_{n}$ at $f_{n}\left(q_{n}\right)=e$ tends to zero as $n \rightarrow \infty$. Thus, $\hat{f}(\widehat{\Sigma})$ is tangent to $\widehat{\mathbb{H}}$ at $\widehat{f}(\widehat{p})=e$. In particular, the left invariant Gauss map of $\widehat{f}$ at $\widehat{p}$ lies in $\Upsilon \cup(-\Upsilon)$. Furthermore, $\widehat{f}$ is everywhere tangent to some right invariant hyperbolic Killing field $\widehat{K}$, by observation (P2) ( $\widehat{K}$ is the limit of appropriate rescalings of the $K_{n}$ after extracting a subsequence, as we did in (6.1)). In particular, $\widehat{K}$ is everywhere tangent to $\widehat{\mathbb{H}}$, which implies that $\widehat{K}$ is generated by a 1-parameter subgroup of $\widehat{\mathbb{H}}$. Let $\widehat{d}: X \rightarrow \mathbb{R}$ stand for the oriented distance function associated to the foliation $\widehat{\mathcal{F}}$. Note that $\widehat{d}$ is constant along each of the integral curves of $\widehat{K}$. By Corollary 5.9, $\widehat{f}(\widehat{\Sigma})$ is contained in one side of $\widehat{\mathbb{H}}$, i.e., the restriction of $\widehat{d}$ to $\widehat{f}(\widehat{\Sigma})$ is nonpositive or nonnegative. Moreover, arguing as in the proof of Claim 6.4 there exists a small compact topological disk $\widehat{D}=\widehat{f}\left(B_{\widehat{\Sigma}}(\widehat{p}, \varepsilon)\right)$ which is foliated by compact arcs of integral curves of $\widehat{K}$, such that:

(Q) Exactly one of these compact arcs of integral curves of $\widehat{K}$ in $\widehat{D}$ lies in the zero set of $\widehat{d}$, while all the other ones lie in $\widehat{d}^{-1}(0, \infty)\left(\right.$ or in $\left.\widehat{d}^{-1}(-\infty, 0)\right)$.

Since $f_{n}$ converges to $\widehat{f}$, then the sequence of disks $D_{n}:=f_{n}\left(B_{\Sigma}\left(q_{n}, \varepsilon\right)\right)$ converges smoothly to $\widehat{D}$. As $\left\{\mathbb{H}_{n}\right\}_{n} \rightarrow \widehat{\mathbb{H}}$, then we can assume that the distance function $d_{n}: \widetilde{\mathrm{SL}}(2, \mathbb{R}) \rightarrow \mathbb{R}$ associated to the foliation $\mathcal{F}_{n}$ converges on compact sets of $\widetilde{\mathrm{SL}}(2, \mathbb{R})$ to $\widehat{d}$. As $K_{n}$ is everywhere tangent to the leaves of $\mathcal{F}_{n}$, we conclude that $d_{n}$ is constant along the integral curves of $K_{n}$. Furthermore, item $(\mathrm{Q})$ and the previous convergence properties ensure that for $n$ large enough, $D_{n}$ contains an integral curve segment of $K_{n}$ along which $d_{n}$ has a local extremum. Hence, there are points $r_{n} \in B_{\Sigma}\left(q_{n}, \varepsilon\right)$ such that $f_{n}$ is tangent at $f_{n}\left(r_{n}\right)$ to some leaf of $\mathcal{F}_{n}$. Therefore, $f$ is tangent at $f\left(r_{n}\right)$ to some leaf $L_{a}$ of $\mathcal{F}$. By Corollary 5.9, we deduce that $f(\Sigma)$ lies on one side of $L_{a}$. Once here, and using similar ideas, we can adapt the last four paragraphs of the proof of Claim 6.4 to our $\widetilde{S L}(2, \mathbb{R})$ setting, and conclude that $f(\Sigma)$ is tangent to another leaf $L_{b}$ of $\mathcal{F}$, and lies in the topological slab of $\widetilde{\operatorname{SL}}(2, \mathbb{R})$ bounded by $L_{a} \cup L_{b}$. This proves item 1 of Claim 6.6, and item 2 is a direct consequence of item 1, Corollary 5.9 and the connectedness of $\Sigma$ that we leave to the reader.

As for item 3 of Claim 6.6, since the leaves of $\mathcal{F}$ are minimal, the mean curvature comparison principle applied to $f(\Sigma)$ and $L_{a}, L_{b}$ implies that the mean curvature vector of $f(\Sigma)$ points towards the interior of the previously defined topological slab at the tangency points between $f(\Sigma)$ and $L_{a} \cup L_{b}$. 
From here, a straightforward continuity argument applied to the normal field to $\mathcal{F}$ gives that $\gamma_{f}$ intersects both $\Upsilon$ and $-\Upsilon$. If $\Sigma$ is an annulus, then $\gamma_{f}$ is clearly a closed curve. Otherwise, $\Sigma$ is simply connected and $f(\Sigma)$ is tangent to $L_{a}$ along at least two (in fact, infinitely many) distinct integral curves of $\widetilde{K}_{\Sigma}=f^{*}\left(K_{\Sigma}\right)$. This implies that $\gamma_{f}$ takes the same value at these integral curves of $\widetilde{K}_{\Sigma}$. As $\gamma$ is embedded, we now conclude that $\gamma_{f}$ is a closed curve.

Assertion 6.5 follows from the Slab Property (Claim 6.6) and our discussion in the paragraph just before Claim 6.6.

We now complete the proof of Theorem 6.1. Since $X$ is not isomorphic to $\mathrm{SU}(2)$, it is either isomorphic to a semidirect product or it is isomorphic to $\widetilde{\mathrm{SL}}(2, \mathbb{R})$. By Assertions 6.2, 6.3 and 6.5, $\gamma_{f}$ is a simple closed curve, which completes the proof of Theorem 6.1.

Corollary 6.7 Let $f:(\Sigma, p) \rightarrow(X, e)$ be a pointed limit immersion that satisfies the hypotheses stated just before Theorem 6.1.

(1) If $\Sigma$ is diffeomorphic to an annulus, then it has linear area growth.

(2) If $\Sigma$ is simply connected, then one of the two following possibilities holds:

(a) f factors through a pointed immersion $\widehat{f}:(\widehat{\Sigma}, \widehat{p}) \rightarrow(X, e)$ of an annulus. In this case, $\Sigma$ has quadratic area growth and the constant mean curvature immersion $\widehat{f}$ is stable.

(b) $f$ is periodic, in the sense that there exists an element $a_{\Sigma} \in X-\Gamma_{\Sigma}$ such that the left translation by $a_{\Sigma}$ leaves $f(\Sigma)$ invariant.

Proof Recall that $f(\Sigma)$ is foliated by integral curves of $K_{\Sigma}$. Since $X$ is a simply connected Lie group diffeomorphic to $\mathbb{R}^{3}$, then the integral curves of $K_{\Sigma}$ are proper Jordan arcs. Let $\widetilde{K}_{\Sigma}=f^{*}\left(K_{\Sigma}\right)$ denote the Killing field induced on $\Sigma$ by $K_{\Sigma}$ after pulling back $K_{\Sigma}$ through $f$.

Assume that $\Sigma$ is diffeomorphic to an annulus and we will show that item 1 holds. Consider the natural projection $\Pi_{\Gamma_{\Sigma}}: X \rightarrow X / \Gamma_{\Sigma}$, where $X / \Gamma_{\Sigma}$ denotes the quotient surface whose points are the integral curves of $K_{\Sigma}$ in $X$. Since $\Sigma$ is an annulus, then $\left(\Pi_{\Gamma_{\Sigma}} \circ f\right)(\Sigma)$ is an immersed closed curve $\beta \subset X / \Gamma_{\Sigma}$ and $f: \Sigma \rightarrow \beta$ is a trivial $\mathbb{R}$-bundle over $\beta$, whose fibers are the integral curves of $\widetilde{K}_{\Sigma}$. Take a global section of this trivial bundle. The image set of this global section is an embedded closed curve $\alpha \subset \Sigma$ which intersects transversely each of the integral curves of $\widetilde{K}_{\Sigma}$. Since $\widetilde{K}_{\Sigma}$ is a Killing vector field, then the image $\psi_{t}(\alpha)$ of $\alpha$ through the flow $\left\{\psi_{t} \mid t \in \mathbb{R}\right\}$ by isometries of $\Sigma$ associated to $\widetilde{K}_{\Sigma}$ produces a foliation of $\Sigma$ by curves isometric to $\alpha$, so in particular all of these curves $\psi_{t}(\alpha)$ have the same length. From here it is straightforward to show that $\Sigma$ has linear area growth, so item 1 is proved.

Next suppose that $\Sigma$ is simply connected. Consider the quotient space $\Sigma / \widetilde{K}_{\Sigma}$ of integral curves of $\widetilde{K}_{\Sigma}$. Thus, $\Sigma / \widetilde{K}_{\Sigma}$ is a 1-dimensional manifold 
diffeomorphic to $\mathbb{R}$, and there is a natural projection $\Pi: \Sigma \rightarrow \Sigma / \widetilde{K}_{\Sigma}$. We can identify $\Sigma / \widetilde{K}_{\Sigma}=\mathbb{R}$, so that $\Pi(p)=0$ for the base point $p$ of $\Sigma$ satisfying $f(p)=e$. Let $\widehat{G}: \mathbb{R}=\Sigma / \widetilde{K}_{\Sigma} \rightarrow \gamma_{f} \subset \mathbb{S}^{2}$ be the mapping such that $G=\widehat{G} \circ \Pi$, where $G$ stands for the left invariant Gauss map of $f$. Since $\gamma_{f}$ is a simple closed regular curve by Theorem 6.1 , it is easy to check that the map $\widehat{G}$ is the universal cover of $\gamma_{f}$. Let $\tau: \Sigma / \widetilde{K}_{\Sigma} \rightarrow \Sigma / \widetilde{K}_{\Sigma}$ be one of the two generators of the group of automorphisms of the covering $\widehat{G}: \Sigma / \widetilde{K}_{\Sigma} \rightarrow \gamma_{f} \subset \mathbb{S}^{2}$. Since the left invariant Gauss map determines the surface up to left translations by [26, Theorem 3.7], given a point $q \in \Pi^{-1}(\tau(0))$, the left translation $l_{f(q)}: X \rightarrow X$ induces a nontrivial isometry $\tilde{l}_{q}: \Sigma \rightarrow \Sigma$ that is a lift of $\tau$ to $\Sigma$, i.e., $\tau \circ \Pi=\Pi \circ \widetilde{l_{q}}$, and satisfies $\widetilde{l_{q}}(p)=q$. Note that $\widetilde{l_{q}}$ acts freely on $\Sigma$ and the quotient $\widehat{\Sigma}=\Sigma / \widetilde{l_{q}}$ is an annulus. We now have two possibilities.

(R1) $f\left(\Pi^{-1}(0)\right)=f\left(\Pi^{-1}(\tau(0))\right)$, in which case $f$ factors through the quotient annulus $\widehat{\Sigma}$ to an immersion $\widehat{f}: \widehat{\Sigma} \rightarrow X$.

(R2) $f\left(\Pi^{-1}(0)\right) \neq f\left(\Pi^{-1}(\tau(0))\right)$, in which case the integral curves $f\left(\Pi^{-1}(0)\right), f\left(\Pi^{-1}(\tau(0))\right)$ of $K_{\Sigma}$ are disjoint.

In any of the two possibilities above, the isometry group Iso $(\Sigma)$ of the induced metric on $\Sigma$ by $f$ contains an $\mathbb{R}$-type subgroup (which corresponds to left translations by any element in $\Gamma_{\Sigma}$ ) and a $\mathbb{Z}$-type subgroup (which corresponds to the subgroup generated by $\widetilde{l_{q}}$ ), such that these two subgroups generate a subgroup $\Lambda$ of $\operatorname{Iso}(\Sigma)$, although $\Lambda$ is not necessarily isomorphic to $\mathbb{R} \times \mathbb{Z}$, because elements in the $\mathbb{R}$-type subgroup do not necessarily commute with those in the $\mathbb{Z}$-subgroup; also note that $\Sigma$ would have at most quadratic area growth provided that $\Lambda$ is isomorphic to $\mathbb{R} \times \mathbb{Z}$.

If case (R1) holds, then the quotient metric on $\widehat{\Sigma}$ (which is the induced metric by $\widehat{f}$ ) has linear area growth, and so $\Sigma$ has quadratic area growth. Since $f$ is stable and the cover $\Sigma \rightarrow \widehat{\Sigma}$ is cyclic, then the quotient immersion $\widehat{f}: \widehat{\Sigma} \rightarrow X$ is also stable (see for instance [29, Proposition 2.5]), which proves that item 2a in the statement of Corollary 6.7 holds. Finally, if case (R2) holds, then we define $a_{\Sigma}=f(q)$, which lies in $X-\Gamma_{\Sigma}$ since $f(p)=e \in \Gamma_{\Sigma}$ and the integral curves $f\left(\Pi^{-1}(0)\right), f\left(\Pi^{-1}(\tau(0))\right)$ of $K_{\Sigma}$ are disjoint. Then, the left translation by $a_{\Sigma}$ leaves $f(\Sigma)$ invariant and we have item $2 \mathrm{~b}$ of the corollary. Now the proof is complete.

\section{Proof of Theorem 1.5 when $X$ is a semidirect product}

In this section we will prove Theorem 1.5 in the case that $X$ is a semidirect product $\mathbb{R}^{2} \rtimes_{A} \mathbb{R}$ endowed with its canonical metric.

Let $S_{n}$ be a sequence of constant mean curvature spheres in $X$ with Area $\left(S_{n}\right)>n$ for all $n$. In order to prove Theorem 1.5 in $X$ it suffices to consider the case where all the spheres $S_{n}$ lie in the connected component $\mathcal{C}$ of 
the space of index-one spheres in $X$ with $H>h_{0}(X)$ given in Proposition 3.4. Indeed, if Theorem 1.5 holds for any such sequence of spheres $\left(S_{n}\right)_{n} \subset \mathcal{C}$, the discussion after Definition 3.5 ensures that $h_{0}(X)=H(X)$, and hence any $H$-sphere $\Sigma$ in $X$ satisfies that $H>h_{0}(X)$, and so Theorem 1.5 holds in full generality.

So, assuming these conditions (in particular, that $S_{n} \in \mathcal{C}$ for every $n$ ), Proposition 5.6, Theorem 6.1 and Corollary 6.7 imply that there exists a pointed limit immersion $f:(\Sigma, p) \leftrightarrow(X, e)$ of the spheres $S_{n}$ with the following properties:

(S1) $f$ is complete, has constant mean curvature $h_{0}(X)$, it is stable and its left invariant Gauss map image $\gamma_{f}$ is either a point or a regular, simple closed curve in $\mathbb{S}^{2} \subset T_{e} X$.

(S2) $f$ is everywhere tangent to a nonzero, right invariant Killing vector field $K_{\Sigma}$ in $X$. Moreover, exactly one of the following three situations happens:

(S2.1) $\Sigma$ is diffeomorphic to an annulus, and it has linear area growth.

(S2.2) $\Sigma$ is simply connected and $f: \Sigma \uparrow X$ factors through an immersion $\widehat{f}: \widehat{\Sigma} \rightarrow X$ of an annulus. In this case, $\Sigma$ has quadratic area growth and $\widehat{f}$ is a stable immersion with constant mean curvature.

(S2.3) There exists $a \in X-\Gamma_{\Sigma}$ such that the left translation by $a$ leaves $f(\Sigma)$ invariant. Here, $\Gamma_{\Sigma}$ is the 1-parameter subgroup of $X$ that generates $K_{\Sigma}$.

Remark 7.1 In case (S2.2), we have that $f: \Sigma \rightarrow X$ is an infinite covering of the topological annulus $f(\Sigma)$. In case (S2.1), $\Sigma$ is a topological annulus itself, but the immersion $f: \Sigma \rightarrow X$ can be a finite covering of the topological annulus $f(\Sigma)$.

Recall that if $\gamma_{f}$ is a point, then $f(\Sigma)$ is a two-dimensional subgroup of $X$. In particular $f(\Sigma)$ is an entire Killing graph with respect to some right invariant vector field in $X$, and so Theorem 1.5 holds. Hence, from now on we will assume that $f(\Sigma)$ is not a two-dimensional subgroup; in particular, $\gamma_{f}$ is a regular Jordan curve in $\mathbb{S}^{2}$, and $K_{\Sigma}$ is unique (up to scaling) among right invariant vector fields on $X$ everywhere tangent to $f(\Sigma)$.

First assume that the matrix $A$ is singular. By Lemma 4.5, $X$ is isometric to $\mathbb{R}^{3}$ or to some $\mathbb{E}(\kappa, \tau)$ space with $\kappa \leq 0$. So, $X$ has isometry group of dimension 6 or 4 and, as explained at the end of the introduction, Theorem 1.5 holds for $X$.

So, from now on, we will assume that the matrix $A$ is regular. By the previous discussion, the next result directly implies that Theorem 1.5 holds for any metric semidirect product $X$, as desired.

Theorem 7.2 In the above conditions, $K_{\Sigma}$ is horizontal, i.e., $K_{\Sigma} \in \operatorname{Span}\left\{F_{1}, F_{2}\right\}$, and $f(\Sigma)$ is an entire Killing graph in $X$ with respect to any nonzero right 
invariant Killing vector field $V \in \operatorname{Span}\left\{F_{1}, F_{2}\right\}$ that is linearly independent from $K_{\Sigma}$ (with the notation of (4.5)).

Observe that if Theorem 7.2 holds, then case (S2.3) occurs for $f$ (because in cases (S2.1) and (S2.2) the image surface $f(\Sigma)$ is an immersed annulus in $X$, which contradicts that $f(\Sigma)$ is an entire graph).

We will divide the proof of Theorem 7.2 into four steps. In Sect. 7.1 we will prove that Theorem 7.2 holds when $K_{\Sigma} \in \operatorname{Span}\left\{F_{1}, F_{2}\right\}$; thus, Theorem 7.2 will be proved provided that we find a contradiction whenever $K_{\Sigma}$ is not horizontal. Assuming that $K_{\Sigma} \notin \operatorname{Span}\left\{F_{1}, F_{2}\right\}$, in Sects. 7.2, 7.3, 7.4 we will respectively show that cases (S2.3), (S2.2), (S2.1) above cannot occur. This will complete the proof of Theorem 7.2 (and thus of Theorem 1.5 for $X$ a metric semidirect product).

\subsection{Proof of Theorem 7.2 when $K_{\Sigma}$ is horizontal}

Lemma 7.3 If $K_{\Sigma} \in \operatorname{Span}\left\{F_{1}, F_{2}\right\}$, then $f$ is an entire Killing graph with respect to any right invariant vector field $V \in \operatorname{Span}\left\{F_{1}, F_{2}\right\}$ that is linearly independent from $K_{\Sigma}$.

Proof In this case, $K_{\Sigma}=\lambda \partial_{x}+\mu \partial_{y}$ for some $\lambda, \mu \in \mathbb{R}$. Since $f(\Sigma)$ is foliated by integral curves of $K_{\Sigma}$, then $f(\Sigma)$ is ruled by straight lines in the direction of $K_{\Sigma}$. By Assertion 6.3, we conclude that the lemma will hold provided that $\gamma_{f}$ does not pass through the North or South pole of $\mathbb{S}^{2}$. Arguing by contradiction, assume that $\gamma_{f}$ passes through the North or South pole of $\mathbb{S}^{2}$. Then, $f$ is in the conditions of Claim 6.4, which ensures that $f(\Sigma)$ is contained in a smallest slab-type region $\mathbb{R}^{2} \rtimes_{A}[a, b]$ with $a \leq 0 \leq b, a \neq b, f(\Sigma)$ intersects each of the planes $\mathbb{R}^{2} \rtimes_{A}\{a\}, \mathbb{R}^{2} \rtimes_{A}\{b\}$ in nonempty sets whose components are integral curves of $K_{\Sigma}$, and $f(\Sigma)$ transversely intersects every intermediate plane $\mathbb{R}^{2} \rtimes_{A}\{z\}$ with $a<z<b$. Let $Y$ be the flat Riemannian product $\mathbb{R}^{2} \times[a, b]$. Thus, we can consider $f: \Sigma \rightarrow Y$ to be a complete flat immersion, which is ruled by straight lines parallel to $K_{\Sigma}$. In particular, $\Sigma$ has quadratic area growth with respect to the pullback metric of $Y$ through $f$. Since the coefficients of the canonical metric on $\mathbb{R}^{2} \rtimes_{A} \mathbb{R}$ with respect to the $(x, y, z)$-coordinates only depend on $z$ by Eq. (4.6), we conclude that the identity map Id: $Y \rightarrow \mathbb{R}^{2} \rtimes_{A}[a, b]$ is a quasi-isometry. From here, we deduce that $\Sigma$ has at most quadratic area growth with respect to the pullback metric of $X$ through $f$.

Let $V$ be any horizontal right invariant vector field on $X$ that is linearly independent from $K_{\Sigma}$. As the coefficients of the canonical metric are bounded in every horizontal slab of finite width by Eq. (4.6), then $V$ is bounded in $\mathbb{R}^{2} \rtimes_{A}[a, b]$. Denoting by $N$ the unit normal vector to $f$, we deduce that $J:=\langle N, V\rangle$ is a bounded Jacobi function on $\Sigma$ that changes sign (changes of 
sign of $J$ occur at points of $\left.f^{-1}\left(\mathbb{R}^{2} \rtimes_{A}\{a, b\}\right)\right)$. As $f: \Sigma \rightarrow X$ is a complete stable $h_{0}(X)$-surface with at most quadratic area growth, then the conformal structure of $\Sigma$ is parabolic, and Corollary 1 in Manzano et al. [21] implies that $J$ cannot change sign, which is a contradiction. This contradiction completes the proof of the lemma.

\subsection{Case (S2.3) is impossible when $K_{\Sigma}$ is not horizontal}

Assume that $K_{\Sigma} \notin \operatorname{Span}\left\{F_{1}, F_{2}\right\}$. The next lemma reduces this case to the specific nonhorizontal, right invariant vector field $K_{\Sigma}=F_{3}$ given by (4.5), which is generated by the 1-parameter subgroup of $X$ corresponding to the $z$ axis. In proving the next lemma, we will be making use of the assumption that $A$ is a regular matrix, as discussed just before the statement of Theorem 7.2.

Lemma 7.4 If $K_{\Sigma} \notin \operatorname{Span}\left\{F_{1}, F_{2}\right\}$, then after a horizontal left translation of $f$ and a scaling of $K_{\Sigma}$, the following statements hold:

(1) $K_{\Sigma}=F_{3}$.

(2) Case (S2.3) does not happen.

Proof Since $K_{\Sigma} \notin \operatorname{Span}\left\{F_{1}, F_{2}\right\}$, then after scaling and using (4.3) and (4.5),

$$
\begin{aligned}
K_{\Sigma} & =F_{3}+s \partial_{x}+t \partial_{y}=(a x+b y+s) \partial_{x}+(c x+d y+t) \partial_{y}+\partial_{z} \\
& \equiv\left(\begin{array}{c}
A \mathbf{p}+\mathbf{q} \\
1
\end{array}\right),
\end{aligned}
$$

for some $s, t \in \mathbb{R}$, where $\mathbf{p}=(x, y), \mathbf{q}=(s, t)$. Given $\mathbf{P}_{0}=\left(\mathbf{p}_{0}, 0\right) \in$ $\mathbb{R}^{2} \rtimes_{A}\{0\}$, the right invariant vector field $\left(l_{\mathbf{P}_{0}}\right)_{*}\left(K_{\Sigma}\right)$ is everywhere tangent to the left translation of $f$ by $\mathbf{P}_{0}$. Hence, to prove item 1 of the lemma it suffices to find $\mathbf{p}_{0} \in \mathbb{R}^{2}$ such that $\left(l_{\mathbf{P}_{0}}\right)_{*}\left(K_{\Sigma}\right)=F_{3}$. By direct computation using the group operation in $\mathbb{R}^{2} \rtimes_{A} \mathbb{R}$, see Sect. 4.2 , we have that $l_{\mathbf{P}_{0}}(\mathbf{p}, z)=\left(\mathbf{p}_{0}+\mathbf{p}, z\right)$ is an Euclidean translation, hence its differential is the identity after identifying the tangent spaces of $X$ at both points $(\mathbf{p}, z),\left(\mathbf{p}_{0}+\mathbf{p}, z\right)$ with $\mathbb{R}^{3}$ in the basis $\partial_{x}, \partial_{y}, \partial_{z}$. Therefore, the value of $\left(l_{\mathbf{P}_{0}}\right)_{*}\left(K_{\Sigma}\right)$ at $l_{\mathbf{P}_{0}}(\mathbf{p}, z)$ is given by

$$
\left(d l_{\mathbf{P}_{0}}\right)_{(\underset{z}{\mathbf{p}})}\left(\begin{array}{c}
A \mathbf{p}+\mathbf{q} \\
1
\end{array}\right)=\left(\begin{array}{c}
A \mathbf{p}+\mathbf{q} \\
1
\end{array}\right)
$$

while

$$
\left.\left(F_{3}\right)_{l_{\mathbf{p}_{0}}(\mathbf{p}}\right)=\left(\begin{array}{c}
A\left(\mathbf{p}_{0}+\mathbf{p}\right) \\
1
\end{array}\right)
$$

hence we only need to define $\mathbf{p}_{0}$ such that $A \mathbf{p}_{0}=\mathbf{q}$, which can be done since $A$ is regular. This proves the first statement in the lemma. 
To prove the second assertion, assume that case (S2.3) holds with $K_{\Sigma}=F_{3}$. Hence, there exists $a \in X-\Gamma_{\Sigma}=X-\{z$-axis $\}$ such that the left translation by $a$ leaves $f(\Sigma)$ invariant. Writing $a=\left(\mathbf{p}_{0}, z_{0}\right) \in \mathbb{R}^{2} \rtimes_{A} \mathbb{R}$ as the product $\left(\mathbf{p}_{0}, 0\right) *\left(\mathbf{0}, z_{0}\right)$ and using that $\left(\mathbf{0}, z_{0}\right) \in \Gamma_{\Sigma}$, we conclude that $a$ can be chosen so that $z_{0}=0$. As $l_{a}$ leaves $f(\Sigma)$ invariant and $F_{3}$ is unique up to rescaling among right invariant vector fields which are nonzero and everywhere tangent to $f(\Sigma)$, then $\left(l_{a}\right)_{*}\left(F_{3}\right)=\lambda F_{3}$ for some $\lambda \in \mathbb{R}$. But the value at $l_{a}(\mathbf{p}, z)=\left(\mathbf{p}_{0}+\mathbf{p}, z\right)$ of $\left(l_{a}\right)_{*}\left(F_{3}\right)$ is

$$
\left(d l_{a}\right)_{\left(\begin{array}{c}
\mathbf{p} \\
z
\end{array}\right)}\left(\begin{array}{c}
A \mathbf{p} \\
1
\end{array}\right)=\left(\begin{array}{c}
A \mathbf{p} \\
1
\end{array}\right),
$$

while $\left(F_{3}\right)_{l_{a}(\underset{z}{\mathbf{p}})}$ is given by (7.1). Since $A$ is a regular matrix, then we deduce that $\left(l_{a}\right)_{*}\left(F_{3}\right)$ cannot be a multiple of $F_{3}$. This completes the proof.

\subsection{Case (S2.2) is impossible when $K_{\Sigma}$ is not horizontal}

Assume that $K_{\Sigma} \notin \operatorname{Span}\left\{F_{1}, F_{2}\right\}$ and that case (S2.2) above holds. By item 1 of Lemma 7.4, we can assume after a horizontal left translation that $K_{\Sigma}=F_{3}$. This of course may produce a change of base point for $f$, of the form $f(p):=$ $Q \in \mathbb{R}^{2} \rtimes_{A}\{0\}$. Recall from the beginning of Sect. 7 that $f:(\Sigma, p) \rightarrow(X, e)$ is obtained as a limit of pointed limit immersions $f_{n}:\left(S_{n}, p_{n}\right) \rightarrow(X, e)$ of spheres $S_{n} \in \mathcal{C}$. Applying to the $f_{n}$ the same horizontal left translation that we applied to $f$ in order to have $K_{\Sigma}=F_{3}$, we have $f_{n}\left(p_{n}\right)=Q$ for all $n$.

By Proposition 3.4, each sphere $S_{n} \in \mathcal{C}$ is Alexandrov embedded. In this way, for each $n \in \mathbb{N}$ there exists a three-dimensional Riemannian manifold $\left(B_{n}, g_{n}\right)$ which is topologically a closed ball and a Riemannian submersion $\widetilde{f}_{n}:\left(B_{n}, g_{n}\right) \rightarrow X$ such that $\partial B_{n}=S_{n},\left.\widetilde{f}_{n}\right|_{S_{n}}=f_{n}$ and $\partial B_{n}$ is mean convex.

Lemma 7.5 In the conditions above, there is some $R^{*}>0$ and points $q_{n} \in S_{n}$ such that the volumes of the Riemannian metric balls $B_{g_{n}}\left(q_{n}, R^{*}\right)$ in $\left(B_{n}, g_{n}\right)$ of radius $R^{*}$ centered at $q_{n}$ tend to $\infty$ as $n \rightarrow \infty$.

Proof As explained in Remark 4.2, the surface $f(\Sigma)$ can be obtained by pulling back via $\Pi_{K_{\Sigma}}$ a closed immersed curve $\alpha$ contained in $X / K_{\Sigma}$. Since in our case $K=K_{\Sigma}=F_{3}$ and every integral curve of $F_{3}$ intersects any horizontal plane $\mathbb{R}^{2} \rtimes_{A}\left\{z_{0}\right\}$ transversely at a single point, we can identify $X / K_{\Sigma}$ with $\mathbb{R}^{2} \rtimes_{A}\{0\}$.

Consider the vertical geodesic of $X$

$$
\Gamma=\{\Gamma(t)=(0,0, t) \mid t \in \mathbb{R}\} .
$$

Given $r>0$, let $\mathcal{W}(\Gamma, r)$ denote the solid metric cylinder of radius $r$ around $\Gamma$, i.e., the set of points of $X$ whose distance to $\Gamma$ is at most $r$. Since $f(\Sigma)$ is an 
annulus invariant under the flow of $F_{3}$, and since all cylinders $\mathcal{W}(\Gamma, r)$ are also invariant under this flow, it is clear that $f(\Sigma)$ is contained in a solid metric cylinder $\mathcal{W}_{0}:=\mathcal{W}\left(\Gamma, r_{0}\right)$ for some $r_{0}>0$, such that $\partial \mathcal{W}_{0}$ is at a positive distance from $f(\Sigma)$.

We next recall the following independent a priori radius estimate for compact stable minimal surfaces with boundary in $X=\mathbb{R}^{2} \rtimes_{A} \mathbb{R}$, obtained by the authors in [25, Theorem 1.1]: there exists some $R=R\left(r_{0}\right)>0$ associated to the solid metric cylinder $\mathcal{W}_{0} \subset X$, with the following property:

( $\star$ Let $M$ be any compact, stable minimal surface in $X$ whose boundary is contained inside $\mathcal{W}_{0}$. Then, the intrinsic radius ${ }^{3}$ of $M$ is less than $R$.

In particular, there are no complete stable minimal surfaces contained in $\mathcal{W}_{0}$. In our current setting, this has the consequence that $h_{0}(X)>0$. Indeed, recall that the surface $f(\Sigma)$ is complete, stable, it has constant mean curvature equal to $h_{0}(X)$, and it is contained in $\mathcal{W}_{0}$. Thus, it cannot be minimal, by $(\star)$; therefore, $h_{0}(X)>0$.

Let us also consider the positive number $\mu_{0}=\mu_{0}\left(r_{0}\right)$ defined in the following way. Observe that $\mathcal{W}_{0}$ is foliated by integral curves of $F_{3}$. We define $\mu_{0}$ as the maximum length of all the arcs of integral curves of $F_{3}$ that are contained in the compact piece of $\mathcal{W}_{0}$ that lies in the slab determined by the planes $\mathbb{R}^{2} \rtimes_{A}\{R+2\}$ and $\mathbb{R}^{2} \rtimes_{A}\{-R-2\}$; note that $\mu_{0}$ exists since $\mathcal{W}_{0} \cap\left[\mathbb{R}^{2} \rtimes_{A}\{0\}\right]$ is compact.

As $\Sigma$ is simply connected and $f: \Sigma \rightarrow X$ is invariant under the flow of $F_{3}$, then, by the discussion in Remark 4.2, we may parameterize $f$ as

$$
f(s, t)=l_{\Gamma(t)}(\alpha(s)), \quad(s, t) \in \mathbb{R}^{2},
$$

where $\alpha=\alpha(s): \mathbb{R} \rightarrow \mathbb{R}^{2} \rtimes_{A}\{0\}$ is a curve parameterized by arc length in $\mathbb{R}^{2} \rtimes_{A}\{0\}$, and so that under the natural identification $\Sigma \equiv\left\{(s, t) \in \mathbb{R}^{2}\right\}$, the base point $p \in \Sigma$ for $f$ corresponds to $(0,0)$. In particular, $f(0,0)=\alpha(0)=$ $Q$. Note that since we are in the conditions of case (S2.2), the image $\alpha(\mathbb{R})$ is an immersed closed curve and $\alpha(s)$ is $L$-periodic, where $L$ is the length of $\alpha(\mathbb{R})$.

For each $s_{0} \in \mathbb{R}$ we may define numbers $c^{-}\left(s_{0}\right)<0, c^{+}\left(s_{0}\right)>0$ such that

$$
A\left(s_{0}\right):=\left\{l_{\Gamma(t)}\left(\alpha\left(s_{0}\right)\right) \mid c^{-}\left(s_{0}\right) \leq t \leq c^{+}\left(s_{0}\right)\right\}
$$

is the arc of the integral curve of $F_{3}$ that passes through $\alpha\left(s_{0}\right)$ whose endpoints lie in $\mathbb{R}^{2} \rtimes_{A}\{R+2\}$ and $\mathbb{R}^{2} \rtimes_{A}\{-R-2\}$. Note that $A\left(s_{0}\right) \subset f(\Sigma)$, and that the length of $A\left(s_{0}\right)$ is at most $\mu_{0}$.

\footnotetext{
3 The radius of a compact Riemannian manifold $M$ with boundary is the maximum distance of points in $M$ to its boundary $\partial M$.
} 


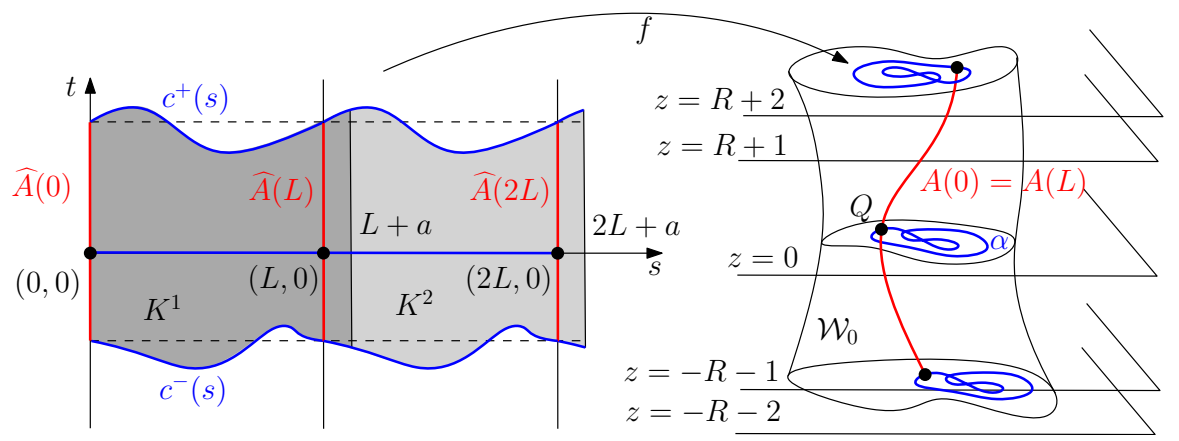

Fig. 6 Left: the compact regions $K^{1} \subset K^{2}$ in the $(s, t)$-plane. The vertical segments $\widehat{A}(0)$, $\widehat{A}(L), \widehat{A}(2 L)$ apply through $f$ into the compact arc $A(0)$, which is part of the integral curve of $F_{3}$ passing through $Q$. Each $K^{j}$ wraps $j$ times through $f$ around its image, due to the $L$-periodicity of $f$ in the $s$-variable

Take some $a \in(0, L)$ and consider for each $j \in \mathbb{N}$ the compact region of $\Sigma$ (viewed as $\mathbb{R}^{2}$ with coordinates $(s, t)$ ) given by

$$
K^{j}=\left\{(s, t) \mid 0 \leq s \leq j L+a, c^{-}(s) \leq t \leq c^{+}(s)\right\},
$$

see Fig. 6.

Since $f$ is a limit surface of the $f_{n}$, it follows that for $n$ large enough, there exist compact simply connected domains $K_{n}^{j} \subset S_{n}$ such that $f_{n}\left(K_{n}^{j}\right) \subset \mathcal{W}_{0}$ and the sequence $\left\{\left.f_{n}\right|_{K_{n}^{j}}\right\}_{n}$ converges to $\left.f\right|_{K^{j}}$ uniformly as $n \rightarrow \infty$. For $\varepsilon>0$ sufficiently small and less than the injectivity radius of $\Sigma$, denote by $\mathcal{U}^{k}$ the closed geodesic disk in $\Sigma$ of radius $\varepsilon$ centered at $(k L, 0)$ with $k \in\{1, \ldots, j\}$. After choosing $\varepsilon>0$ sufficiently small, we may assume that $\left\{\mathcal{U}^{1}, \ldots, \mathcal{U}^{j}\right\}$ forms a pairwise disjoint collection, and the intrinsic distance between $\mathcal{U}^{i}, \mathcal{U}^{k}$ for $i, k \in\{1, \ldots, j\}, i \neq k$, is greater than $2 \delta$ for some $\delta>0$ independent of $j$. Also, there exist closed geodesic disks of radius $\varepsilon, \mathcal{U}_{n}^{k} \subset K_{n}^{j}$, such that $\left\{\left.f_{n}\right|_{\mathcal{U}_{n}^{k}}\right\}_{n}$ converges to $\left.f\right|_{\mathcal{U}^{k}}$ uniformly as $n \rightarrow \infty$. Observe that for each $j$, there exists $n_{0}(j) \in \mathbb{N}$ such that if $n \geq n_{0}(j)$, the related compact disks $\mathcal{U}_{n}^{k} \subset K_{n}^{j}$ satisfy that the intrinsic distance between $\mathcal{U}_{n}^{i}$ and $\mathcal{U}_{n}^{k}$ inside $K_{n}^{j}$ is greater than $\delta$ for every $i, k \in\{1, \ldots, j\}, i \neq k$. It is important to notice that $\delta$ does not depend on $j$, since $f(s, t)$ is $L$-periodic with respect to $s$.

Let $I$ denote a small compact segment in $\mathbb{R}^{2} \rtimes_{A}\{0\}$ centered at $Q$ and transversal to $f(\Sigma)$ at $Q$. Let $\alpha_{n} \subset S_{n}$ denote the set $f_{n}^{-1}\left(\mathbb{R}^{2} \rtimes_{A}\{0\}\right)$. Since $f$ is a limit of the $f_{n}$ and $f(\Sigma)$ intersects $\mathbb{R}^{2} \rtimes_{A}\{0\}$ transversely, then by the Transversality Lemma (Lemma 5.3), $\alpha_{n}$ is a closed simple curve in $S_{n}$, for $n$ large enough; to see this, note that $\mathbb{R}^{2} \rtimes_{A}\{0\}$ is a two-dimensional subgroup of $X$. As $f(s, t)$ is $L$-periodic in the variable $s$, the previous convergence properties imply that for $n=n(j)$ large enough there exist $j$ points 
$q_{n}^{1}, \ldots, q_{n}^{j} \in \alpha_{n} \cap K_{n}^{j}$ such that, for every $k=1, \ldots, j$, we have $q_{n}^{k} \in \mathcal{U}_{n}^{k}$ and $f_{n}\left(q_{n}^{k}\right) \in I$. In particular, we note for later use the following property:

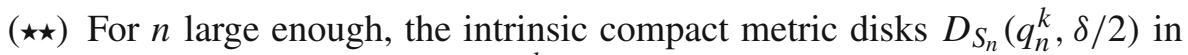
$S_{n}$ of radius $\delta / 2$ and center $q_{n}^{k}, k=1, \ldots, j$, are pairwise disjoint.

Let $J_{n}^{k}$ be the compact connected arc of $\alpha_{n}$ contained in $K_{n}^{j}$ whose endpoints are $q_{n}^{1}$ and $q_{n}^{k}$, for each $k \in\{1, \ldots, j\}$. Let $W_{n}$ be the vector field on $S_{n}$ obtained by pulling back via $f_{n}$ the tangent part to $f_{n}\left(S_{n}\right)$ of the right invariant vector field $K_{\Sigma}=F_{3}$; clearly, $W_{n}$ has no zeros on $K_{n}^{j} \subset S_{n}$ for $n$ large enough, since $K_{\Sigma}$ has no zeros, is everywhere tangent to $f(\Sigma)$ and $\left\{\left.f_{n}\right|_{K_{n}^{j}}\right\}_{n}$ converges to $\left.f\right|_{K^{j}}$ uniformly as $n \rightarrow \infty$. Moreover, again for $n$ large enough, the integral curves of $W_{n}$ that start at any point of any of the arcs $J_{n}^{k}$ satisfy that their images by $f_{n}$ intersect both planes $\mathbb{R}^{2} \rtimes_{A}\{-R-1\}$ and $\mathbb{R}^{2} \rtimes_{A}\{R+1\}$. In particular, we can define the compact disks $D_{n}^{k} \subset S_{n}$ obtained by letting the arc $J_{n}^{k}$ flow under $W_{n}$ between the sets $f_{n}^{-1}\left(\mathbb{R}^{2} \rtimes_{A}\{-R-1\}\right)$ and $f_{n}^{-1}\left(\mathbb{R}^{2} \rtimes_{A}\{R+1\}\right)$ of $S_{n}$. In fact, $D_{n}^{k} \subset K_{n}^{j}$ for all $k \in\{1, \ldots, j\}$ and for $n$ large.

Let $\gamma_{n}^{k}:=\partial D_{n}^{k}$, which is a Jordan curve in $S_{n}$. Observe that $\gamma_{n}^{k}$ can be written as a union $A_{1}^{n} \cup A_{2}^{n} \cup B_{1}^{n} \cup B_{2}^{n}$, where:

(T1) $A_{1}^{n}, A_{2}^{n}$ are compact arcs of integral curves of $W_{n}$ that pass through $q_{n}^{1}$ and $q_{n}^{k}$, respectively. In particular, the endpoints of both $f_{n}\left(A_{1}^{n}\right)$ and $f_{n}\left(A_{2}^{n}\right)$ lie on the planes $\mathbb{R}^{2} \rtimes_{A}\{-R-1\}, \mathbb{R}^{2} \rtimes_{A}\{R+1\}$. Moreover, since $f_{n}\left(A_{1}^{n}\right)$ and $f_{n}\left(A_{2}^{n}\right)$ converge uniformly as $n \rightarrow \infty$ to proper subarcs of the compact arc $A(0)$ defined by equation (7.4), then the lengths of $A_{1}^{n}$ and $A_{2}^{n}$ are smaller than the constant $\mu_{0}$ for $n$ large enough.

(T2) The arc $f_{n}\left(B_{1}^{n}\right)$ (resp. $f_{n}\left(B_{2}^{n}\right)$ ) lies in the plane $\mathbb{R}^{2} \rtimes_{A}\{-R-1\}$ (resp. $\left.\mathbb{R}^{2} \rtimes_{A}\{R+1\}\right)$.

Recall that $\left(B_{n}, g_{n}\right)$ is the abstract Riemannian three-ball that $S_{n}$ bounds, and $\tilde{f}_{n}:\left(B_{n}, g_{n}\right) \rightarrow X$ is a Riemannian submersion with mean convex boundary $\left.\widetilde{f}_{n}\right|_{\partial B_{n}}=f_{n}$. In particular, $S_{n}$ is a good barrier to solve Plateau problems in $B_{n}$ in the sense of Meeks and Yau [33]. More precisely, for each $k \in\{1, \ldots, j\}$ (and $n$ large enough), there exists a compact stable embedded minimal disk $M_{n}^{k} \subset B_{n}$ of least area, with $\partial M_{n}^{k}=\gamma_{n}^{k}$. Let us stress here that $M_{n}^{k}$ is embedded in the abstract ball $B_{n}$; in contrast, the image $\widetilde{f}\left(M_{n}^{k}\right) \subset X$ of $M_{n}^{k}$ via the Riemannian submersion $\widetilde{f}$ might be non-embedded in $X$. Furthermore, also by [33], $M_{n}^{k}$ is an immersion transverse to $\partial B_{n}$ at the regular points of its boundary $\gamma_{n}^{k}$ (this transversality property does not hold at the vertices of $\gamma_{n}^{k}$ ). In particular, $M_{n}^{k}$ is an immersion transverse to $\partial B_{n}$ at the interior points of the $\operatorname{arcs} A_{1}^{n}$ and $A_{2}^{n}$. Therefore, if we denote by $\Lambda_{n} \subset B_{n}$ the embedded surface $\left\{q \in B_{n} \mid \widetilde{f}_{n}(q) \in \mathbb{R}^{2} \rtimes_{A}\{0\}\right\}$ and $\beta_{n}^{k}:=M_{n}^{k} \cap \Lambda_{n}$, then $\beta_{n}^{k} \cap \partial M_{n}^{k}=\left\{q_{n}^{1}, q_{n}^{k}\right\}$, and the intersection of $M_{n}^{k}$ with $\Lambda_{n}$ is clearly transverse at $q_{n}^{1}, q_{n}^{k}$ since the arcs $A_{1}^{n}, A_{2}^{n}$ are transverse to $\Lambda_{n}$. Moreover, up to a small perturbation of $\Lambda_{n}$ in $B_{n}$ 


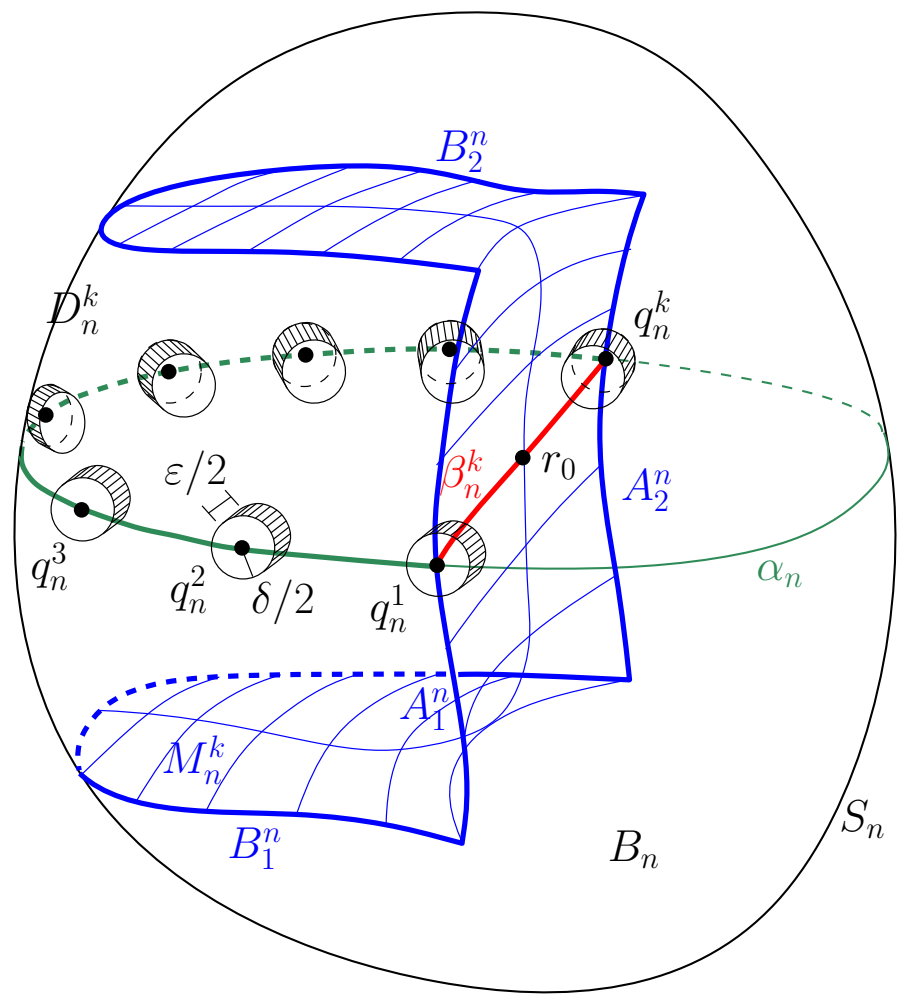

Fig. 7 The least area disk $M_{n}^{k}$ inside the abstract Riemannian ball $B_{n}$ has the same boundary $\gamma_{n}^{k}$ as the disk $D_{n}^{k} \subset S_{n}=\partial B_{n} \cdot \gamma_{n}^{k}$ consists of four consecutive $\operatorname{arcs} A_{1}^{n}, B_{1}^{n}, A_{2}^{n}, B_{2}^{n}$ satisfying properties (T1), (T2)

that fixes its boundary, we may assume that the intersection of $M_{n}^{k}$ with $\Lambda_{n}$ is transverse. Therefore, $\beta_{n}^{k}$ is a (possibly disconnected) compact 1-dimensional manifold with boundary, and a connected component of $\beta_{n}^{k}$ is a compact arc in $B_{n}$ joining $q_{n}^{1}$ with $q_{n}^{k}$. We will keep denoting this compact arc by $\beta_{n}^{k}$, see Fig. 7.

Take $r \in \beta_{n}^{k}$. We next give a lower bound of $d_{X}\left(\widetilde{f}_{n}(r), f_{n}\left(B_{1}^{n} \cup B_{2}^{n}\right)\right)$, the distance in $X$ from $\widetilde{f}_{n}(r)$ to $f_{n}\left(B_{1}^{n} \cup B_{2}^{n}\right)$. Since $f_{n}\left(B_{1}^{n} \cup B_{2}^{n}\right)$ is contained in the union of planes $\mathbb{R}^{2} \rtimes_{A}\{\underset{\widetilde{f}}{(}(R+1)\}$, then $d_{X}\left(\widetilde{f}_{n}(r), f_{n}\left(B_{1}^{n} \cup B_{2}^{n}\right)\right) \geq$ $d_{X}\left(\widetilde{f}_{n}(r), \mathbb{R}^{2} \rtimes_{A}\left\{ \pm R_{1}\right\}\right)$. As $\widetilde{f}_{n}(r)$ lies at an arbitrarily small distance of $\mathbb{R}^{2} \rtimes_{A}\{0\}$ and $d_{X}\left(\mathbb{R}^{2} \rtimes_{A}\{0\}, \mathbb{R}^{2} \rtimes_{A}\{ \pm(R+1)\}\right)=R+1$ (this follows, for instance, from [28, Lemma 3.9], since $\mathbb{R}^{2} \rtimes_{A}\{ \pm(R+1)\}$ are right cosets of $\left.\mathbb{R}^{2} \rtimes_{A}\{0\}\right)$, then $d_{X}\left(\widetilde{f}_{n}(r), \mathbb{R}^{2} \rtimes_{A}\{ \pm(R+1)\}\right)$ can be taken arbitrarily close to $R+1$. In particular,

$$
d_{X}\left(\widetilde{f_{n}}(r), f_{n}\left(B_{1}^{n} \cup B_{2}^{n}\right)\right)>R, \quad \text { for all } r \in \beta_{n}^{k} .
$$


As $\left.\widetilde{f}_{n}\right|_{M_{n}^{k}}: M_{n}^{k} \uparrow X$ is by construction a compact immersed stable minimal disk in $X$ whose boundary is contained in the solid metric cylinder $\mathcal{W}_{0}$, we deduce from the radius estimate $(\star)$ above that for any $r \in \beta_{n}^{k}$ the distance $d_{M_{n}^{k}}\left(r, \gamma_{n}^{k}\right)$ in $M_{n}^{k}$ from $r$ to $\gamma_{n}^{k}=\partial M_{n}^{k}$ is not greater than $R$. Note that $d_{M_{n}^{k}}\left(r, B_{1}^{n} \cup B_{2}^{n}\right) \geq d_{X}\left(\widetilde{f}_{n}(r), f_{n}\left(B_{1}^{n} \cup B_{2}^{n}\right)\right)$, which by (7.6) is greater than $R$. Therefore,

$$
d_{M_{n}^{k}}\left(r, \gamma_{n}^{k}\right)=d_{M_{n}^{k}}\left(r, A_{1}^{n} \cup A_{2}^{n}\right), \quad \text { for all } r \in \beta_{n}^{k} .
$$

Moreover, since $q_{n}^{1} \in A_{1}^{n}$ and $q_{n}^{k} \in A_{2}^{n}$, we deduce by the continuity and connectedness of the arc $\beta_{n}^{k}$ that there exists some midpoint $r_{0} \in \beta_{n}^{k}$ such that $d_{M_{n}^{k}}\left(r_{0}, A_{1}^{n}\right)=d_{M_{n}^{k}}\left(r_{0}, A_{2}^{n}\right)$. Thus, we can estimate the distance in $B_{n}$ from $r_{0}$ to $A_{i}^{n}, i=1,2$, by

$$
\begin{array}{rlrl}
d_{B_{n}}\left(r_{0}, A_{i}^{n}\right) & \leq d_{M_{n}^{k}}\left(r_{0}, A_{i}^{n}\right) & & \\
& =d_{M_{n}^{k}}\left(r_{0}, A_{1}^{n} \cup A_{2}^{n}\right) & & \left(r_{0} \text { is a midpoint }\right) \\
& =d_{M_{n}^{k}}\left(r_{0}, \gamma_{n}^{k}\right) & & \text { (by Eq. (7.7)) } \\
& \leq R . &
\end{array}
$$

Therefore,

$$
\begin{aligned}
d_{B_{n}}\left(q_{n}^{1}, q_{n}^{k}\right) & \leq d_{B_{n}}\left(q_{n}^{1}, r_{0}\right)+d_{B_{n}}\left(r_{0}, q_{n}^{k}\right) & & \text { (triangle inequality) } \\
& \leq \mu_{0}+d_{B_{n}}\left(r_{0}, A_{1}^{n}\right)+\mu_{0}+d_{B_{n}}\left(r_{0}, A_{2}^{n}\right) & & \text { (triangle inequality and (T 1) } \left.7 \gamma_{0} 9\right) \\
& \leq 2 \mu_{0}+2 R . & & \text { (inequality (7.8)) }
\end{aligned}
$$

Recall that, by item 5 of Proposition 3.4, the norms of the second fundamental forms of the spheres $S_{n}$ are uniformly bounded by some constant $C>0$. Also, note that since we have already proved that $h_{0}(X)>0$ in this case, the values $H_{n}$ of the mean curvatures of $S_{n}$ are uniformly bounded away from zero (since $H_{n}>h_{0}(X)$ for every $n$ ). Hence, by [30], there exists some $\varepsilon_{1}>0$ smaller than the injectivity radius of $B_{n}$ such that $S_{n}=\partial B_{n}$ has a regular neighborhood $\mathcal{V}_{n}^{\varepsilon_{1}}$ in $B_{n}$ of fixed size $\varepsilon_{1}$ (independent of $n$ ). In other words, we have a diffeomorphism

$$
\Phi_{n}: S_{n} \times\left[0, \varepsilon_{1}\right) \rightarrow \mathcal{V}_{n}^{\varepsilon_{1}}, \quad \Phi(x, t)=\exp _{x}^{n}\left(t N_{n}(x)\right)
$$

where $N_{n}$ is the inward pointing unit normal in $\left(B_{n}, g_{n}\right)$ of the mean convex sphere $S_{n}=\partial B_{n}$ and $\exp ^{n}$ denotes the exponential map in $\left(B_{n}, g_{n}\right)$.

By Property ( $\star$ ) above, for $n$ large enough, the solid cylinders $C_{n}^{1}, \ldots, C_{n}^{j}$ in $\mathcal{V}_{n}^{\varepsilon_{1}}$ given by the image under $\Phi_{n}$ of the compact cylinders $D_{S_{n}}\left(q_{n}^{k}, \delta / 2\right) \times$ $\left[0, \varepsilon_{1} / 2\right]$ are mutually disjoint, see Fig. 7 . Also, note that the volume of each $C_{n}^{k}$ in $\left(B_{n}, g_{n}\right)$ is greater than some $V>0$, independent of $n, j$ and $k$ (again this follows from the uniform bound of the second fundamental forms of the 
$S_{n}$, as well as from the fact $\varepsilon_{1}$ does not depend on $n$ ). In particular, for $n$ large enough, the total volume in $\left(B_{n}, g_{n}\right)$ of the (disjoint) union $C_{n}^{1} \cup \cdots \cup C_{n}^{j}$ is at least $j V$. Note that this volume can be made arbitrarily large, as $j \in \mathbb{N}$ was chosen fixed but arbitrary.

Also, by the triangle inequality we have for any $x \in C_{n}^{k}, k \in\{1, \ldots, j\}$, that $d_{B_{n}}\left(q_{n}^{k}, x\right) \leq \frac{\varepsilon_{1}+\delta}{2}$. This inequality and (7.9) imply that

$$
d_{B_{n}}\left(q_{n}^{1}, x\right) \leq d_{B_{n}}\left(q_{n}^{1}, q_{n}^{k}\right)+d_{B_{n}}\left(q_{n}^{k}, x\right) \leq 2\left(\mu_{0}+R\right)+\frac{\varepsilon_{1}+\delta}{2},
$$

from where we deduce that $C_{n}^{1} \cup \cdots \cup C_{n}^{j}$ is contained in the Riemannian ball of $\left(B_{n}, g_{n}\right)$ centered at $q_{n}^{1}$ and of radius $R^{*}:=2\left(\mu_{0}+R\right)+\frac{\varepsilon_{1}+\delta}{2}$.

In particular, we have proved the following: given a fixed but arbitrary $j \in \mathbb{N}$, then for all $n \in \mathbb{N}$ large enough, the metric ball $B_{g_{n}}\left(q_{n}^{1}, R^{*}\right)$ in the Riemannian manifold $\left(B_{n}, g_{n}\right)$ centered at $q_{n}^{1} \in \partial B_{n}$ of radius $R^{*}$ has volume at least $j V$, where $V, R^{*}>0$ do not depend on $j, n$. This clearly proves Lemma 7.5.

We will next obtain a contradiction with Lemma 7.5, that will prove that case (S2.2) is impossible when $K_{\Sigma} \notin \operatorname{Span}\left\{F_{1}, F_{2}\right\}$, which was the objective of the present Sect. 7.3. First, observe that as the second fundamental forms of the $f_{n}$ are uniformly bounded, there exists $\delta>0$ such that, for all $n$, the following properties hold:

(U1) $\left(B_{n}, g_{n}\right)$ can be extended to a compact Riemannian three-manifold with boundary $\left(\widetilde{B}_{n}, \widetilde{g}_{n}\right)$, in the sense that $B_{n}$ is a compact subset of $\widetilde{B}_{n}$ and $\left.\widetilde{g}_{n}\right|_{B_{n}}=g_{n}$.

(U2) The map $(x, t) \in S_{n} \times[-\delta, 0] \rightarrow \exp _{x}^{n}\left(t N_{n}(x)\right) \in \widetilde{B}_{n}-\operatorname{Int}\left(B_{n}\right)$ is a diffeomorphism, where $\exp ^{n}$ denotes the exponential map of $\left(\widetilde{B}_{n}, \widetilde{g}_{n}\right)$ and $N_{n}$ is the unit normal vector to $S_{n}$ at $x$ with respect to $\widetilde{g}_{n}$ such that $H_{n} N_{n}$ is the mean curvature vector of $S_{n}$.

(U3) The metrics $\widetilde{g}_{n}$ are uniformly bounded in the $C^{2}$-topology.

Let $\varphi:(-\delta, 0] \rightarrow(0, \infty)$ be a smooth positive function such that $\varphi=1$ in $[-\delta / 3,0]$ and $\varphi(t)=\frac{1}{\tilde{B}^{t+\delta}}$ in $(-\delta,-2 \delta / 3]$. We define the complete Riemannian metric $\widehat{g}_{n}$ on $\widetilde{B}_{n}-\partial \widetilde{B}_{n}$ by

$$
\widehat{g}_{n}= \begin{cases}g_{n} & \text { in } B_{n} \\ \varphi^{2} \widetilde{g}_{n} & \text { in } S_{n} \times(-\delta, 0]\end{cases}
$$

(we are identifying $S_{n} \times[-\delta, 0]$ with $\widetilde{B}_{n}-\operatorname{Int}\left(B_{n}\right)$ through the diffeomorphism that appears in property (U2)). Observe that

$$
\widehat{g}_{n}=\frac{1}{(t+\delta)^{2}} \widetilde{g}_{n} \text { in } S_{n} \times(-\delta,-2 \delta / 3] .
$$


As $\left.\widetilde{g}_{n}\right|_{\partial \widetilde{B}_{n}}$ is a Riemannian metric on the closed surface $\partial \widetilde{B}_{n}$, then (7.10) implies that given $\varepsilon>0$, there exists $\delta_{1}=\delta_{1}(\varepsilon) \in(2 \delta / 3, \delta)$ such that the restriction of $\widehat{g}_{n}$ to $S_{n} \times\left(-\delta,-\delta_{1}\right]$ is $\varepsilon$-close in the $C^{2}$-topology to the hyperbolic metric of constant sectional curvature -1 . In particular, the Ricci curvature of $\widehat{g}_{n}$ satisfies

$$
\left|\operatorname{Ric}_{\widehat{g}_{n}}+2\right|_{L^{\infty}\left(S_{n} \times\left(-\delta,-\delta_{1}\right]\right)}<b(\varepsilon),
$$

where $b(\varepsilon)$ can be made arbitrarily small for $\varepsilon$ small.

As the coefficients of the Ricci tensor of $\varphi^{2} \widetilde{g}_{n}$ are smooth expressions of the coefficients of $\widetilde{g}_{n}$, of those of the Ricci tensor of $\widetilde{g}_{n}$ and of $\varphi, \varphi^{\prime}, \varphi^{\prime \prime}$, then property (U3) and the smoothness of $\varphi$ imply that the Ricci curvature of $\varphi^{2} \widetilde{g}_{n}$ satisfies for all $n$ :

$$
\operatorname{Ric}_{\varphi^{2} \widetilde{g}_{n}} \text { is uniformly bounded in } S_{n} \times\left[-\delta_{1}, 0\right] \text {. }
$$

As $g_{n}$ is locally homogeneous, (7.11) and (7.12) imply that there exist $c<0$ independent of $n$ such that $\operatorname{Ric}_{\widehat{g}_{n}} \geq 2 c$ in $\widetilde{B}_{n}-\partial \widetilde{B}_{n}$ for all $n$. Since $\left(\widetilde{B}_{n}-\partial \widetilde{B}_{n}, \widehat{g}_{n}\right)$ is a complete Riemannian manifold, then Bishop's comparison theorem gives the following upper bound for the volume of the metric ball $B_{\widehat{g}_{n}}\left(q_{n}, R^{*}\right)$ in $\left(\widetilde{B}_{n}-\partial \widetilde{B}_{n}, \widehat{g}_{n}\right)$ of radius $R^{*}>0$ centered at $q_{n}$ :

$$
\operatorname{Vol}\left(B_{\widehat{g}_{n}}\left(p_{n}, R^{*}\right)\right) \leq V\left(c, R^{*}\right)
$$

where $V\left(c, R^{*}\right)$ denotes the volume of any metric ball of radius $R^{*}$ in the three-dimensional space form of constant sectional curvature $c$. This is a contradiction with Lemma 7.5, as desired.

\subsection{Case (S2.1) is impossible when $K_{\Sigma}$ is not horizontal}

Assume that case (S2.1) holds and $K_{\Sigma} \notin \operatorname{Span}\left\{F_{1}, F_{2}\right\}$. We start with the same normalizations as in the previous Sect. 7.3. So, $K_{\Sigma}=F_{3}$, and $f:(\Sigma, p) \rightarrow$ $(X, Q)$ (here $\left.Q \in \mathbb{R}^{2} \rtimes_{A}\{0\}\right)$ is obtained as a limit of pointed immersions $f_{n}:\left(S_{n}, p_{n}\right) \rightarrow(X, Q)$ of spheres $S_{n} \in \mathcal{C}$. Also, we will consider the compact Riemannian balls $\left(B_{n}, g_{n}\right)$ with $\partial B_{n}=S_{n}$, and Riemannian submersions $\widetilde{f}_{n}:\left(B_{n}, g_{n}\right) \rightarrow X$ such that $\partial B_{n}$ is mean convex and $\left.\widetilde{f}_{n}\right|_{\partial B_{n}}=f_{n}$. Let $\alpha_{n}:=f_{n}^{-1}\left(\mathbb{R}^{2} \rtimes_{A}\{0\}\right) \subset S_{n}$, which is a simple closed curve in $S_{n}$ for $n$ large enough, by the Transversality Lemma (Lemma 5.3). Also, let $K_{\Sigma}^{n}$ be the Killing field on $B_{n}$ that is induced by $K_{\Sigma}$ via the pullback by the submersion $\widetilde{f}_{n}$. 
Lemma 7.6 In the conditions above, $\alpha_{\infty}:=f^{-1}\left(\mathbb{R}^{2} \rtimes_{A}\{0\}\right) \subset \Sigma$ is a simple closed curve in $\Sigma$, and the sequence $\left\{\left.f_{n}\right|_{\alpha_{n}}\right\}_{n}$ converges uniformly to the immersion $\left.f\right|_{\alpha_{\infty}}$.

Proof Recall that in this case (S2.1), $\Sigma$ is an annulus. Also, recall that the space $X / K_{\Sigma}$ whose points are the integral curves of $K_{\Sigma}=F_{3}$ can be identified with $\mathbb{R}^{2} \rtimes_{A}\{0\}$; see the beginning of the proof of Lemma 7.5. In particular, by Remark 4.2, $f(\Sigma)$ is the immersed annulus obtained by letting the immersed closed curve $\beta:=f(\Sigma) \cap\left(\mathbb{R}^{2} \rtimes_{A}\{0\}\right)$ flow under $F_{3}$. Also, by the argument in the proof of item 1 of Corollary 6.7, we have that $\alpha_{\infty}:=f^{-1}\left(\mathbb{R}^{2} \rtimes_{A}\{0\}\right)$ is an embedded closed curve in $\Sigma$ that generates the homology group of the cylinder $\Sigma$. Note that $\left.f\right|_{\alpha_{\infty}}: \alpha_{\infty} \rightarrow \mathbb{R}^{2} \rtimes_{A}\{0\}$ is not an embedding, and it could actually be a finite covering of the closed immersed curve $\beta=f\left(\alpha_{\infty}\right)$.

Finally, the convergence property of $\left\{\left.f_{n}\right|_{\alpha_{n}}\right\}_{n}$ to $\left.f\right|_{\alpha_{\infty}}$ follows from the convergence of the pointed immersions $f_{n}:\left(S_{n}, p_{n}\right) \rightarrow(X, Q)$ to $f:(\Sigma, p) \rightarrow$ $(X, Q)$, and the compactness of $\alpha_{\infty}$ in $\Sigma$.

By Theorems 1 and 2 in [33], $\alpha_{n}$ is the boundary of a smooth, embedded least-area disk $M(n)$ in the abstract Riemannian 3-ball $B_{n}$ and this disk is transverse to $S_{n}=\partial B_{n}$ along its boundary $\alpha_{n}$. Note that, even though $M(n)$ is embedded in $B_{n}, \widetilde{f}_{n}(M(n))$ might be non-embedded in $X$. Also, note that the intrinsic radii of the disks $M(n)$ are bounded by a universal constant $R>0$ by Theorem 1.1 in [25], since their compact image boundary curves $\left.f_{n}\right|_{\partial M(n)}=$ $\left.f_{n}\right|_{\alpha_{n}}$ converge uniformly as $n \rightarrow \infty$ to $\left.f\right|_{\alpha_{\infty}}$, and $f\left(\alpha_{\infty}\right)$ is contained in a solid metric cylinder around $\Gamma=\{(0,0, t) \mid t \in \mathbb{R}\}$ (so the same holds for $f_{n}(\partial M(n))$ for all $n \in \mathbb{N}$, for a slightly larger solid metric cylinder around $\left.\Gamma\right)$.

Notice that each of the immersed curves $\alpha_{n}$ is the boundary curve of the intrinsically flat "horizontal" disk in $D_{n} \subset B_{n}$. Since the lengths of the curves $\alpha_{n}$ are uniformly bounded, then the areas of the disks $D_{n}$ are also uniformly bounded by the classical isoperimetric inequality for flat disks. In particular, the areas of the least-area disks $M(n)$ are also uniformly bounded. It follows that a subsequence of the immersed stable minimal disks $\widetilde{f}_{n}(M(n)) \subset X$, which lie locally in the mean convex side of $f_{n}\left(S_{n}\right)$ near $f_{n}\left(\alpha_{n}\right)$, converge uniformly on compact sets to an immersed stable minimal disk $M(\infty)$ in $X$ with boundary $f\left(\alpha_{\infty}\right)$. We claim that $M(n)$ lies in the piecewise smooth, closed upper half-ball $B_{n}^{+}=\widetilde{f}_{n}^{-1}\left(\mathbb{R}^{2} \rtimes_{A}[0, \infty)\right)$ determined by the disk $\widetilde{f}_{n}^{-1}\left(\mathbb{R}^{2} \rtimes_{A}\{0\}\right) \subset B_{n}$. To see this, first recall that for every $z_{0} \in \mathbb{R}$, the halfspace of the form $\mathbb{R}^{2} \rtimes_{A}\left[z_{0}, \infty\right)$ is mean convex in $X$, and the constant mean curvature of its boundary is equal to $H(X) \geq 0$; recall that if $\operatorname{tr}(A)=0$, then $H(X)=0$ and so both half-spaces limited by $\mathbb{R}^{2} \rtimes_{A}\left\{z_{0}\right\}$ are mean convex. Since $f_{n}\left(\alpha_{n}\right) \subset \mathbb{R}^{2} \rtimes_{A}\{0\}$ is the boundary of the immersed minimal disk $f_{n}(M(n)$ ), then the maximum principle (or the mean curvature comparison 
principle) applied to $M(n)$ and to the smooth surfaces $\widetilde{f}_{n}^{-1}\left(\mathbb{R}^{2} \rtimes_{A}\{z\}\right), z \in \mathbb{R}$, implies that $M(n) \subset B_{n}^{+}$as desired.

We next claim that there exists an $\eta \in(0, \pi / 4)$ such that the angle that $M(n)$ makes with $S_{n}$ along $\alpha_{n}$ is greater than $\eta$ for all $n \in \mathbb{N}$ large enough. Assume that the claim does not hold; in this case, $M(\infty)$ is tangent to $f(\Sigma)$ at some boundary point $Q^{\prime} \in \partial M(\infty)$. As $M(\infty)$ lies locally in the mean convex side of $f(\Sigma)$ along $f\left(\alpha_{\infty}\right)$, then the Hopf boundary maximum principle implies that $f(\Sigma)$ coincides with $M(\infty)$ nearby $Q^{\prime}$. This is a contradiction since we know that $h_{0}(X)>0$ in this case, as it was explained just after property ( $\star$ ) in the previous section. Thus, the claim at the beginning of this paragraph holds.

Let $N_{M(n)}$ denote the unit normal vector field to $M(n)$ in $\left(B_{n}, g_{n}\right)$. We claim that for $n$ sufficiently large, $g_{n}\left(N_{M(n)}, K_{\Sigma}^{n}\right)$ has no zeros along $\alpha_{n}$. Arguing by contradiction, suppose that after passing to a subsequence, $K_{\Sigma}^{n}$ is tangent to $M(n)$ at some point $q_{n} \in \alpha_{n}$ for all $n$. Then $K_{\Sigma}^{n}\left(q_{n}\right)$ and the unit tangent vector $w_{n}$ to $\alpha_{n}$ at $q_{n}$ generate the tangent space $T_{q_{n}} M(n)$. By the last paragraph, the angle between $T_{q_{n}} M(n)$ and $T_{q_{n}} S_{n}$ is greater than $\eta$. Since $K_{\Sigma}$ is everywhere tangent to $f(\Sigma)$, then the angle that $K_{\Sigma}^{n}\left(q_{n}\right)$ makes with $T_{q_{n}} S_{n}$ tends to zero as $n \rightarrow \infty$. Since $K_{\Sigma}^{n}\left(q_{n}\right)$ is tangent to $M(n)$ at $q_{n}$, then the angle that $K_{\Sigma}^{n}\left(q_{n}\right)$ makes with $w_{n}$ becomes arbitrarily small if $n$ is large enough (note that $\left.w_{n} \in T_{q_{n}} S_{n} \cap T_{q_{n}} M(n)\right)$. Applying the differential of $\widetilde{f}_{n}$ at $q_{n}$, we deduce that the angle between $\left(K_{\Sigma}\right) f_{n}\left(q_{n}\right)=\left(F_{3}\right)_{f_{n}\left(q_{n}\right)}$ and $\left(d f_{n}\right)_{q_{n}}\left(w_{n}\right)$ tends to zero as $n \rightarrow \infty$. Since $\left(d f_{n}\right)_{q_{n}}\left(w_{n}\right)$ is a nonzero horizontal vector, this only can occur provided that $\left(F_{3}\right) f_{n}\left(q_{n}\right)$ becomes horizontal as $n \rightarrow \infty$, or equivalently, the ratio between the component of $\left(F_{3}\right)_{f_{n}\left(q_{n}\right)}$ in the direction of $\partial_{z}$ and its horizontal component tends to zero. By Eq. (4.5), this only can occur if $f_{n}\left(q_{n}\right)$ diverges in $\mathbb{R}^{2} \rtimes_{A}\{0\}$. But this contradicts the fact that $f\left(\alpha_{\infty}\right)$ lies at a finite distance from the origin. Hence the claim follows.

Therefore, after replacing by a subsequence and choosing $N_{M(n)}$ so that the Jacobi function $J_{n}=g_{n}\left(N_{M(n)}, K_{\Sigma}^{n}\right)$ is positive along $\alpha_{n}$, we deduce from the stability of the minimal disk $M(n)$ that $J_{n}$ is positive on $M(n)$.

Consider the Jacobi function $J_{\infty}=\left\langle N_{M(\infty)}, K_{\Sigma}\right\rangle$ defined on the stable minimal disk $M(\infty)$. Observe that $J_{\infty}$ does not vanish at any point of $\partial M(\infty)=f\left(\alpha_{\infty}\right)$ (otherwise $M(\infty)$ would become tangent to $f(\Sigma)$ at some boundary point of $M(\infty)$, which we have seen that contradicts the Hopf boundary maximum principle). Once we know that $J_{\infty}$ does not vanish at any point of $\partial M(\infty)$, then $\left.J_{\infty}\right|_{\partial M(\infty)}$ is positive (because $\left.J_{n}\right|_{\alpha_{n}}$ is positive for $n$ large), and thus, $J_{\infty}>0$ on $M(\infty)$ because $M(\infty)$ is stable.

Consider the mapping $T_{n}: M(n) \times \mathbb{R} \rightarrow X$ given by

$$
T_{n}(q, t)=l_{\Gamma(t)}\left(\tilde{f}_{n}(q)\right)
$$


where $\Gamma(t)$ is given by (7.2). As $J_{n}$ is positive on $M(n), T_{n}$ is a proper submersion of $M(n) \times \mathbb{R}$ into $X$ (note that the projection $(x, t) \mapsto t$ is proper). Our next goal is to take limits of these immersions $T_{n}$. To do this, we will use conformal parameters for $M(n)$ and $M(\infty)$ and a standard three-point condition argument.

Specifically, let $\overline{\mathbb{D}}$ be the closed unit disk in $\mathbb{R}^{2}$ and parameterize each $M(n)$ by a conformal diffeomorphism $\phi_{n}: \overline{\mathbb{D}} \rightarrow M(n)$ in such a way that the points $(1,0),(0,1),(-1,0)$ of $\partial \mathbb{D}$ have as images by $f_{n} \circ \phi_{n}$ three ordered points $w_{1}^{n}, w_{2}^{n}, w_{3}^{n}$ in $f_{n}\left(\alpha_{n}\right)$. Similarly, let $\widetilde{f}_{\infty}: \overline{\mathbb{D}} \rightarrow X$ be the analogous conformal parametrization of $M(\infty)$ with respect to three ordered points $\xi_{1}, \xi_{2}, \xi_{3}$ in $f\left(\alpha_{\infty}\right)$. Since $\left\{\left.f_{n}\right|_{\alpha_{n}}\right\}_{n}$ converges uniformly to $\left.f\right|_{\alpha_{\infty}}$ by Lemma 7.6, we can choose the points $w_{i}^{n}$ so that they converge to $\xi_{i}$, for $i=1,2,3$. With this choice, and taking in mind the a priori radius estimates, the uniform area estimates and the boundary convergence of the minimal disks $D(n)$, we can use a classical argument via the Courant-Lebesgue theorem to ensure that the conformal immersions $\widetilde{f}_{n} \circ \phi_{n}: \overline{\mathbb{D}} \rightarrow X$ converge smoothly up to a subsequence to the conformal immersion $\tilde{f}_{\infty}: \overline{\mathbb{D}} \leftrightarrow X$. Details of this standard compactness argument can be found for instance in [32, pages 423-424], see also [35].

Note that as $n \rightarrow \infty$, the proper immersions $T_{n} \circ\left(\phi_{n} \times 1_{\mathbb{R}}\right): \overline{\mathbb{D}} \times \mathbb{R} \rightarrow X$ converge to the submersion $T_{\infty}: \overline{\mathbb{D}} \times \mathbb{R} \rightarrow X$ given by

$$
T_{\infty}(x, t)=l_{\Gamma(t)}\left(\tilde{f}_{\infty}(x)\right),
$$

which again is a proper submersion since $(x, t) \in \overline{\mathbb{D}} \times \mathbb{R} \rightarrow \mathbb{R} \mapsto t \in \mathbb{R}$ is proper (because $J_{\infty}$ is positive on $M(\infty)$ ). Consider the codimension-one foliation of $\overline{\mathbb{D}} \times \mathbb{R}$ given by the family of compact disks

$$
\mathcal{F}=\left\{D_{t}=T_{\infty}^{-1}\left(\mathbb{R}^{2} \rtimes_{A}\{t\}\right) \mid t \in \mathbb{R}\right\} .
$$

Let $K_{1}$ be the nowhere zero, smooth vector field on $\overline{\mathbb{D}} \times \mathbb{R}$ obtained after pulling back $K_{\Sigma}$ through $T_{\infty}$. Clearly, each of the integral curves of $K_{1}$ in $\overline{\mathbb{D}} \times \mathbb{R}$ intersects the disk $D_{0}$ in a single point. Thus, we can view $\overline{\mathbb{D}} \times \mathbb{R}$ in a natural manner as being the topological product $W:=D_{0} \times \mathbb{R}$, and we endow this three-manifold with boundary with the pulled-back metric by $T_{\infty}$. We will find the desired contradiction as an application of Theorem 9.1 below, as we explain next.

Observe that the boundary $\partial W=\left(\partial D_{0}\right) \times \mathbb{R}$ of $W$ satisfies

$$
\begin{aligned}
T_{\infty}(\partial W) & =\left\{l_{\Gamma(t)}\left(T_{\infty}(x)\right) \mid x \in \partial D_{0}, t \in \mathbb{R}\right\} \\
& =\left\{l_{\Gamma(t)}(f(x)) \mid x \in \alpha_{\infty}, t \in \mathbb{R}\right\}=f(\Sigma) .
\end{aligned}
$$


Recall that $f(\Sigma)$ has constant mean curvature $h_{0}(X)>0$ in $X$ with respect to the unit normal field obtained as the limit of the inward pointing unit normal fields to the spheres $S_{n}$; see the paragraph after property $(\star)$ in Sect. 7.3. Also note that the inward pointing unit normal field on $\partial W$ has positive inner product with the inner conormal to $M(\infty)$ by the Hopf boundary maximum principle, and that we can view $M(\infty)$ as an embedded minimal disk in $W$. Since $M(\infty)$ can be viewed as the limit of the disks $M(n)$, each of which lies in the mean convex ball $B_{n}$ bounded by $S_{n}$, then we conclude that $\partial W$ has constant mean curvature $h_{0}(X)$ with respect to the inward pointing normal field to its boundary (thus, $W$ has mean convex boundary of constant mean curvature).

The vector field $K_{1}$ is clearly a Killing field on $W$ endowed with the pulledback metric by $T_{\infty}$. Note that $K_{1}$ arises from the proper action of $\Gamma=\Gamma(t)$, which we can view as a 1-parameter subgroup of isometries of $W$.

We claim that there exists a nowhere zero Killing field $K_{2}$ in $W$ which is tangent to the foliation $\mathcal{F}$ defined in (7.15), bounded on the end of $W$ given by the end representative $D_{0} \times[0, \infty)$ and such that the closure in $W$ of every integral curve of $K_{2}$ in $\operatorname{Int}(W)$ is a compact arc with end points in $\partial W$. To see this, first note that by Lemma 4.7 there exists a nonzero horizontal right invariant vector field $V$ in $X$ that is bounded in the halfspace $\mathbb{R}^{2} \rtimes_{A}[0, \infty)$. Let $K_{2}$ be the Killing field in $W$ defined as the pullback by $T_{\infty}$ of $V$. Since $V$ is everywhere horizontal and bounded in $\mathbb{R}^{2} \rtimes_{A}[0, \infty)$, then $K_{2}$ is tangent to $\mathcal{F}$ and bounded on the end representative $T_{\infty}^{-1}\left(\mathbb{R}^{2} \rtimes_{A}[0, \infty)\right)$ of $W$. Clearly, both noncompact domains $D_{0} \times[0, \infty), T_{\infty}^{-1}\left(\mathbb{R}^{2} \rtimes_{A}[0, \infty)\right)$ represent the same end of the solid cylinder $W$. Finally, since $T_{\infty}$ is proper, then the closure of every integral curve of $K_{2}$ in $\operatorname{Int}(W)$ is a compact arc in a disk $D_{t}$ for some $t \in \mathbb{R}$, with end points in $\partial W$. Thus our claim in this paragraph is proved.

By Theorem 9.1, the CMC flux (see Definition 9.2)

$$
\operatorname{Flux}\left(\partial W, \partial D_{0} \times\{0\}, K_{1}\right) \neq 0 \text {. }
$$

We will arrive to a contradiction by showing that the above CMC flux is actually zero. Note that $\alpha_{n}$ is homologous to zero in $S_{n}$, and so, the homological invariance of the CMC flux gives that $\operatorname{Flux}\left(\partial B_{n}, \alpha_{n}, K_{\Sigma}^{n}\right)=0$ for all $n$. But this sequence of numbers converges as $n \rightarrow \infty$ to $\operatorname{Flux}\left(\partial W, \partial D_{0} \times\{0\}, K_{1}\right)$, which gives the desired contradiction.

\section{Proof of Theorem 1.5 when $X$ is isomorphic to $\widetilde{\mathrm{SL}}(2, \mathbb{R})$}

In this section we will prove Theorem 1.5 when $X$ is a metric Lie group isomorphic to $\widetilde{\mathrm{SL}}(2, \mathbb{R})$. As explained at the beginning of Sect. 7 , to prove Theorem 1.5 it suffices to prove that if $S_{n}$ is a sequence of constant mean 
curvature spheres in $X$ that lie in the connected component $\mathcal{C}$ of the space of index-one spheres in $X$ with $H>h_{0}(X)$ defined in Proposition 3.4, and for which $\operatorname{Area}\left(S_{n}\right) \rightarrow \infty$ as $n \rightarrow \infty$, then there exists a pointed limit immersion $f:(\Sigma, p) \rightarrow(X, e)$ of this sequence such that $f(\Sigma)$ is an entire Killing graph in $X$ with respect to a right invariant Killing vector field in $X$. Also by the discussion at the beginning of Sect. 7, we can also assume that there exists a pointed limit immersion $f:(\Sigma, p) \rightarrow(X, e)$ of the spheres $S_{n}$ such that properties (S1), (S2) hold. Observe that properties (S1), (S2) and the three mutually exclusive possibilities (S2.1), (S2.3) and (S2.3) make sense in this case when $X$ is isomorphic to $\widetilde{\mathrm{SL}}(2, \mathbb{R})$.

By item 5 of Lemma 4.9, $H(X)>0$. Therefore, by (3.1) we have $h_{0}(X)>$ 0 , which implies that no immersions in $\Delta(f)$ have constant left invariant Gauss map (because two-dimensional subgroups of $X$ are minimal, see Corollary 3.17 in [28]). In particular, $f(\Sigma)$ is not a two-dimensional subgroup of $X$ and the right invariant vector field $K_{\Sigma}$ to which $f(\Sigma)$ is everywhere tangent is unique up to scaling.

Theorem 1.5 when $X$ is isomorphic to $\widetilde{\mathrm{SL}}(2, \mathbb{R})$ will follow directly from the next result.

Theorem 8.1 In the above conditions, $f(\Sigma)$ is an entire Killing graph with respect to a nonzero right invariant vector field on $X$. Moreover, $f$ satisfies the periodicity condition (S2.3) stated at the beginning of Sect. 7.

We next explain the strategy of the proof of Theorem 8.1. Firstly observe that the second sentence of Theorem 8.1 is a consequence of the first one (by the same argument given in the paragraph just after the statement of Theorem 7.2; note that this argument did not use that $X$ was a semidirect product and remains valid in the present case that $X$ is isomorphic to $\widetilde{\mathrm{SL}}(2, \mathbb{R})$ ). So we only need to prove that $f(\Sigma)$ is an entire Killing graph with respect to some nonzero right invariant vector field. The proof of this property will be divided into four steps, depending on the character of the right invariant vector field $K_{\Sigma}$ (elliptic, hyperbolic or parabolic), and on the situations (S2.1), (S2.2) and (S2.3). In Sect. 8.1 we will show that $K_{\Sigma}$ cannot be elliptic. In Sect. 8.2 we will prove that Theorem 8.1 holds if $K_{\Sigma}$ is parabolic. In Sect. 8.3 we will prove that Theorem 8.1 holds if $K_{\Sigma}$ is hyperbolic and case (S2.3) occurs. In Sects. 8.4 and 8.5 we will respectively show that cases (S2.2) and (S2.1) are impossible when $K_{\Sigma}$ is hyperbolic. This discussion exhausts all possible cases, and thus proves Theorem 8.1, and therefore Theorem 1.5 when $X$ is isomorphic to $\widetilde{\mathrm{SL}}(2, \mathbb{R})$. 


\section{1 $K_{\Sigma}$ cannot be elliptic}

Lemma 8.2 $K_{\Sigma}$ is not generated by an elliptic 1-parameter subgroup of $\widetilde{\mathrm{SL}}(2, \mathbb{R})$.

Proof Let $\Gamma^{E}$ be any elliptic 1-parameter subgroup of $\widetilde{\mathrm{SL}}(2, \mathbb{R})$ and let $K^{E}$ be the right invariant, elliptic vector field on $\widetilde{\mathrm{SL}}(2, \mathbb{R})$ generated by the left action of $\Gamma^{E}$ on $\widetilde{\mathrm{SL}}(2, \mathbb{R})$. Each elliptic 1-parameter subgroup of $\widetilde{\mathrm{SL}}(2, \mathbb{R})$ consists of the set of liftings to $\widetilde{\mathrm{SL}}(2, \mathbb{R})$ of all rotations of $\mathbb{H}^{2}$ around a given point; see Sect. 4.3. If $a \in \mathrm{SL}(2, \mathbb{R})$ satisfies $\left(l_{a}\right)_{*}\left(K^{E}\right)=\lambda K^{E}$ for some $\lambda \in \mathbb{R}-\{0\}$, then $a \Gamma^{E} a^{-1}=\Gamma^{E}$, see e.g., the proof of Lemma 4.10. Once here, it is well-known that on an elliptic subgroup this is only possible if $a \in \Gamma^{E}$.

Suppose now that $K_{\Sigma}$ is generated by the left action on $\widetilde{\mathrm{SL}}(2, \mathbb{R})$ of an elliptic 1-parameter subgroup $\Gamma_{\Sigma}=\Gamma^{E}$ of $\widetilde{\mathrm{SL}}(2, \mathbb{R})$ and we will obtain a contradiction. If case (S2.3) holds, then there exists $a \in X-\Gamma_{\Sigma}$ such that $l_{a}(f(\Sigma))=f(\Sigma)$. As the right invariant vector field $\left(l_{a}\right)_{*}\left(K_{\Sigma}\right)$ is everywhere tangent to $l_{a}(f(\Sigma))$ and $K_{\Sigma}$ is unique up to scaling among nonzero right invariant vector fields that are everywhere tangent to $f(\Sigma)$, then we conclude that $\left(l_{a}\right)_{*}\left(K_{\Sigma}\right)=\lambda K_{\Sigma}$ for some $\lambda \in \mathbb{R}, \lambda \neq 0$. Thus, the first paragraph in this proof ensures that $a \in \Gamma^{E}$, a contradiction. Therefore, case (S2.3) cannot hold.

In both cases (S2.1) and (S2.2), Remark 4.2 implies that $f(\Sigma)$ is obtained by the flow under the elliptic right invariant vector field $K_{\Sigma}$ of an immersed closed curve contained in $\widetilde{\mathrm{SL}}(2, \mathbb{R}) / K_{\Sigma}$. In particular, since $K_{\Sigma}$ is Killing, the distance in $X$ of $f(\Sigma)$ to $\Gamma^{E}$ is bounded, or equivalently, $f(\Sigma)$ is contained in a solid metric cylinder in $X$ of fixed radius $r>0$ centered along $\Gamma^{E}$,

$$
\mathcal{W}\left(\Gamma^{E}, r\right)=\left\{x \in X \mid d_{X}\left(x, \Gamma^{E}\right) \leq r\right\},
$$

where $d_{X}$ denotes distance in $X$. As every elliptic 1-parameter subgroup of $X$ contains the center $Z$ of $\widetilde{S L}(2, \mathbb{R})$, then so does $\Gamma^{E}$, from where we deduce that $\mathcal{W}\left(\Gamma^{E}, r\right)$ is invariant under every left translation in $Z$. Also, it is clear that every right invariant vector field of $\widetilde{S L}(2, \mathbb{R})$ descends to the quotient group $\widetilde{\mathrm{SL}}(2, \mathbb{R}) / Z$ given by the action on $\widetilde{\mathrm{SL}}(2, \mathbb{R})$ of the group of left translations by elements of $Z$. As $\mathcal{W}\left(\Gamma^{E}, r\right) / Z$ is compact, the restriction to $\mathcal{W}\left(\Gamma^{E}, r\right)$ of every right invariant vector field in $\widetilde{\mathrm{SL}}(2, \mathbb{R})$ is bounded in $\mathcal{W}\left(\Gamma^{E}, r\right)$.

Let $V$ be any nonzero right invariant vector field in $X$ tangent to $f(\Sigma)$ at $f(p)=e$ and linearly independent from $K_{\Sigma}$. As $V$ is not everywhere tangent to $f(\Sigma)$ and $V$ is bounded in $\mathcal{W}\left(\Gamma^{E}, r\right)$, then the Jacobi function $J=\langle V, N\rangle$ is not identically zero, vanishes at $p$ and it is bounded on $\Sigma$ (here $N$ is the unit normal vector field to $f$ ). Note that $J$ changes sign on $\Sigma$ by the maximum principle. As $f: \Sigma \mapsto X$ is a complete stable $h_{0}(X)$-surface with at most quadratic area growth, the existence of the function $J$ contradicts [21, 
Corollary 1] (see the last paragraph of the proof of Lemma 7.3 for a similar argument). This contradiction proves the lemma.

\subsection{Proof of Theorem 8.1 when $K_{\Sigma}$ is parabolic}

In order to prove Theorem 8.1 if $K_{\Sigma}$ is parabolic, we will use the following auxiliary result:

Lemma 8.3 Let $\Gamma^{P}, \Gamma^{H}$ be two 1-parameter subgroups of $\widetilde{\mathrm{SL}}(2, \mathbb{R})$ such that $\Gamma^{P}$ is parabolic, $\Gamma^{H}$ is hyperbolic and they generate a two-dimensional subgroup of $\widetilde{\mathrm{SL}}(2, \mathbb{R})$. Let $K^{P}, K^{H}$ denote the right invariant vector fields on $\widetilde{\mathrm{SL}}(2, \mathbb{R})$ generated by $\Gamma^{P}, \Gamma^{H}$. Then, there exists a function $h \in$ $C^{\infty}(\widetilde{\mathrm{SL}}(2, \mathbb{R}))$ such that the vector field $K^{H}-h K^{P}$ satisfies:

(1) $K^{H}-h K^{P}$ is bounded in $X=(\widetilde{\mathrm{SL}}(2, \mathbb{R}),\langle\rangle$,$) (regardless of the left$ invariant metric on $\widetilde{\mathrm{SL}}(2, \mathbb{R})$ ).

(2) If the isometry group of $X$ has dimension four, then $K^{H}-h K^{P}$ has constant length.

Proof First note that if we decompose $K^{H}$ as $K^{H}=h K^{P}+V$ for some $h \in C^{\infty}(\widetilde{\mathrm{SL}}(2, \mathbb{R}))$ and some smooth vector field $V$ on $\widetilde{\mathrm{SL}}(2, \mathbb{R})$, then the boundedness of $V$ in $X=(\widetilde{\mathrm{SL}}(2, \mathbb{R}),\langle\rangle$,$) does not depend on the left invariant$ metric $\langle$,$\rangle on \widetilde{\mathrm{SL}}(2, \mathbb{R})$, because all such metrics are quasi-isometric by the identity map in $\widetilde{\mathrm{SL}}(2, \mathbb{R})$. Also, note that the particular bound for $V$ on each specific $X$ might depend on the metric $\langle$,$\rangle . Thus, it suffices to prove item 2$ of the lemma.

We next prove item 2. Let $\mathbb{H}^{2}=\mathbb{H}_{\theta}^{2}$ be the 2-dimensional subgroup of $\widetilde{\mathrm{SL}}(2, \mathbb{R})$ that contains $\Gamma^{P}, \Gamma^{H}$ (see the description of $\mathbb{H}_{\theta}^{2}$ before Lemma 4.11). Consider the Lie group given by the semidirect product $\mathbb{R} \rtimes_{(1)} \mathbb{R}=$ $\{(x, y) \mid x, y \in \mathbb{R}\}$, endowed with the group operation

$$
(x, y) *\left(x^{\prime}, y^{\prime}\right)=\left(x+e^{y} x^{\prime}, y+y^{\prime}\right) .
$$

Let $\Phi_{1}: \mathbb{H}^{2} \rightarrow \mathbb{R} \rtimes_{(1)} \mathbb{R}$ be a group isomorphism, which exists since both $\mathbb{H}^{2}, \mathbb{R} \rtimes_{(1)} \mathbb{R}$ are noncommutative Lie groups of dimension two. As $\Phi_{1}$ is an isomorphism, the push-forward vector fields $\widetilde{K}^{P}=\left(\Phi_{1}\right)_{*}\left(K^{P}\right), \widetilde{K}^{H}=$ $\left(\Phi_{1}\right)_{*}\left(K^{H}\right)$ are nowhere zero and right invariant on $\mathbb{R} \rtimes_{(1)} \mathbb{R}$. Furthermore, as the unique parabolic one-dimensional subgroup of $\mathbb{R} \rtimes_{(1)} \mathbb{R}$ is $\{(x, 0) \mid x \in \mathbb{R}\}$, then we conclude that there exists $\lambda \in \mathbb{R}-\{0\}$ such that $\widetilde{K}^{P}=\lambda \partial_{x}$. Since $\left\{\partial_{x}, x \partial_{x}+\partial_{y}\right\}$ is a basis of right invariant vector fields on $\mathbb{R} \rtimes_{(1)} \mathbb{R}$ and $\lambda \neq 0$, there exist $\mu_{1}, \mu_{2} \in \mathbb{R}$ such that

$$
\widetilde{K}^{H}=\mu_{1} \widetilde{K}^{P}+\mu_{2}\left(x \partial_{x}+\partial_{y}\right)=\left(\mu_{1}+\frac{\mu_{2}}{\lambda} x\right) \widetilde{K}^{P}+\mu_{2} \partial_{y} .
$$


Consider the left invariant metric $\langle$,$\rangle on \mathbb{R} \rtimes_{(1)} \mathbb{R}$ that makes $\Phi_{1}$ an isometry (recall that $\mathbb{H}^{2}$ has the induced metric from $\widehat{S L}(2, \mathbb{R})$ ). As $\partial_{y}$ is left invariant on $\mathbb{R} \rtimes_{(1)} \mathbb{R}$, then the length of $\partial_{y}$ with respect to $\left\langle_{,}\right\rangle_{\widetilde{K}}$ is constant (nonzero). Hence, Eq. (8.2) implies that the length of $\widetilde{K}^{H}-\widetilde{h} \widetilde{K}^{P}$ with respect to $\langle$, is constant, where $\widetilde{h}=\mu_{1}+\frac{\mu_{2}}{\lambda} x \in C^{\infty}\left(\mathbb{R} \rtimes_{(1)} \mathbb{R}\right)$. This implies that if we call $h=\widetilde{h} \circ \Phi_{1} \in C^{\infty}\left(\mathbb{H}^{2}\right)$, then the vector field $\left.\left(K^{H}\right)\right|_{\mathbb{H}^{2}}-\left.h\left(K^{P}\right)\right|_{\mathbb{H}^{2}}$ has constant length on $\mathbb{H}^{2}$.

Parameterize by $\{c(t) \mid t \in \mathbb{R}\}$ the 1-parameter subgroup $\Gamma_{3}$ of $\widetilde{\mathrm{SL}}(2, \mathbb{R})$ generated by $E_{3}$. As the family $\left\{r_{c(t)}\left(\mathbb{H}^{2}\right) \mid t \in \mathbb{R}\right\}$ of right cosets of $\mathbb{H}^{2}$ is a foliation of $\widetilde{\mathrm{SL}}(2, \mathbb{R})$, we can extend $h$ to a smooth function, also called $h \in C^{\infty}(\widetilde{\mathrm{SL}}(2, \mathbb{R}))$, so that $h$ is constant along the orbits of the right action by elements in $\Gamma_{3}$. Suppose now that the left invariant metric on $X$ has isometry group of dimension four. Thus, the right translations $r_{c(t)}$ by elements in $\Gamma_{3}$ are isometries of the ambient metric (because $\Gamma_{3}^{\prime}(0)=\left(E_{3}\right)_{e}$ given by (4.7) and $E_{3}$ is a left invariant Killing vector field of every left invariant metric $\widetilde{\mathrm{SL}}(2, \mathbb{R})$ whose isometry group has dimension four). This property and the right invariance of $K^{H}, K^{P}$ imply that the length of $K^{H}-h K^{P}$ is constant along the orbits of the right action by elements of $\Gamma_{3}$. As $\left.\left(K^{H}\right)\right|_{\mathbb{H}^{2}}-\left.h\left(K^{P}\right)\right|_{\mathbb{H}^{2}}$ has constant length on $\mathbb{H}^{2}$, we conclude that $K^{H}-h K^{P}$ has constant length on $X$ and item 2 of Lemma 8.3 is proved.

The next lemma implies that Theorem 8.1 holds in the case that $K_{\Sigma}$ is parabolic, which is the objective of the present Sect. 8.2.

Lemma 8.4 Suppose that $K_{\Sigma}$ is parabolic. Let $\mathbb{H}_{\theta}^{2}$ be the two-dimensional subgroup of $\widetilde{\mathrm{SL}}(2, \mathbb{R})$ that contains the 1-parameter subgroup $\Gamma_{\Sigma}$ generated by $K_{\Sigma}$. Let $\Gamma^{H}$ be any hyperbolic 1-parameter subgroup of $\mathbb{H}_{\theta}^{2}$ and $K^{H}$ the nonzero right invariant vector field on $\widetilde{\mathrm{SL}}(2, \mathbb{R})$ generated by $\Gamma^{H}$. Then, $f(\Sigma)$ is a smooth entire Killing $K^{H}$-graph in $X=(\widetilde{\mathrm{SL}}(2, \mathbb{R}),\langle\rangle$,$) .$

Proof Consider the foliation $\mathcal{F}$ of $\widetilde{\mathrm{SL}}(2, \mathbb{R})$ by right cosets of $\mathbb{H}_{\theta}^{2}$. As $\Gamma^{H}$ (resp. $\Gamma_{\Sigma}$ ) is a 1-parameter subgroup of $\mathbb{H}_{\theta}^{2}$, then the integral curves of $K^{H}$ (resp. $K_{\Sigma}$ ) are entirely contained in right cosets of $\mathbb{H}_{\theta}^{2}$; see property (P5) just before Claim 6.6. Since $f(\Sigma)$ is ruled by integral curves of $K_{\Sigma}$, we have that if $f(\Sigma)$ intersects a leaf $r_{x}\left(\mathbb{H}_{\theta}^{2}\right)$ of $\mathcal{F}$ at a point $x$, then the integral curve $r_{x}\left(\Gamma_{\Sigma}\right)$ of $K_{\Sigma}$ passing through $x$ is also contained in $f(\Sigma) \cap r_{x}\left(\mathbb{H}_{\theta}^{2}\right)$, and the angle that $f(\Sigma)$ makes with $r_{x}\left(\mathbb{H}_{\theta}^{2}\right)$ along $r_{x}\left(\Gamma_{\Sigma}\right)$ is constant, since left translations by elements of $\Gamma_{\Sigma}$ are isometries of $X$.

Fix one of the leaves of $\mathcal{F}$, say $r_{x}\left(\mathbb{H}_{\theta}^{2}\right), x \in \widetilde{\mathrm{SL}}(2, \mathbb{R})$. Since each of the integral curves of $K^{H}$ contained in $r_{x}\left(\mathbb{H}_{\theta}^{2}\right)$ intersects exactly once the unique integral curve $r_{x}\left(\Gamma_{\Sigma}\right)$ of $K_{\Sigma}$ contained in $r_{x}\left(\mathbb{H}_{\theta}^{2}\right)$, then it follows that Lemma 8.4 will hold provided that we show that $f(\Sigma)$ intersects each of the right cosets $r_{x}\left(\mathbb{H}_{\theta}^{2}\right)$ transversely. 
By straightforward modifications of the arguments in Assertion 6.5 (note that in that assertion, $K_{\Sigma}$ was assumed to be hyperbolic while we are now assuming that $K_{\Sigma}$ is parabolic; nevertheless the arguments in Assertion 6.5 still hold since they will be applied to the foliation by right cosets of $\mathbb{H}_{\theta}^{2}$, which are tangent to both $K_{\Sigma}$ and $K^{H}$ ), we deduce that one of the two following possibilities holds for $f$ :

(V1) $f(\Sigma)$ makes angles with the leaves of $\mathcal{F}$ that are bounded away from zero.

(V2) $f(\Sigma)$ is contained in a smallest slab-type region between two different leaves $L:=r_{x}\left(\mathbb{H}_{\theta}^{2}\right), L^{\prime}:=r_{y}\left(\mathbb{H}_{\theta}^{2}\right)$ of $\mathcal{F}$, for some $x, y \in \widetilde{\mathrm{SL}}(2, \mathbb{R})$, and $f(\Sigma)$ is tangent to $L$ (resp. $L^{\prime}$ ) along an integral curve $\Gamma_{x}$ (resp. $\Gamma_{y}$ ) of $K_{\Sigma}$ (in other words, the conclusions of Claim 6.6 hold).

A straightforward modification of the arguments in the paragraph just before Claim 6.6 implies that if case (V1) holds, then Lemma 8.4 also holds. Therefore, we will assume that case (V2) holds and we will find a contradiction.

Consider the Jacobi function $J=\left\langle K^{H}, N\right\rangle$ on $\Sigma$, where $N$ stands for the unit normal vector to $f$. As $K_{\Sigma}$ is parabolic and everywhere tangent to $f(\Sigma)$, then Lemma 8.3 applied to $K^{P}:=K_{\Sigma}$ and $K^{H}$ ensures that $J$ is bounded on $\Sigma$. Also, note that $J$ vanishes along the curves in $f^{-1}\left(L \cup L^{\prime}\right)$, which exist by condition (V2). Since each nodal domain $\Omega \subset \Sigma$ of $J$ is a smooth infinite strip invariant under a 1-parameter group of isometries (the ones generated by the flow of $K_{\Sigma}$ ), then $\Omega$ has linear area growth. Hence, the closure $\bar{\Omega}$ of $\Omega$ is a parabolic Riemannian surface with boundary, in the sense that there exists a sequence $\left\{\varphi_{j}\right\}_{j} \subset C_{0}^{\infty}(\bar{\Omega})$ such that $0 \leq \varphi_{j} \leq 1$, the $\varphi_{j}^{-1}(1)$ form an increasing exhaustion of $\bar{\Omega}$ and $\int_{\Omega}\left|\nabla \varphi_{j}\right|^{2} \leq \frac{1}{j}$ for each $j$. As $f$ is stable, there exists $v \in C^{\infty}(\Sigma)$ such that $v>0$ and $L v=0$ in $\Sigma$, where $L$ is the Jacobi operator of $f$. Since $J$ is bounded in $\Sigma$ and changes sign, then we have a contradiction with Lemma 8.5 below. This contradiction proves the lemma.

Lemma 8.5 Suppose $\Sigma$ is a complete Riemannian surface without boundary. Suppose that $L=\Delta+P$ is a Schrödinger operator with potential $P \in C^{\infty}(\Sigma)$. Let $u, v \in C^{\infty}(\Sigma)$ satisfying $L u=L v=0$, with $u$ not identically zero and $v>0$ in $\Sigma$. If $\Omega \subset \Sigma$ is a nodal domain of $u$ such that $\bar{\Omega}$ is parabolic and $u$ is bounded in $\Omega$, then $u$ is a nonzero multiple of $v$ and $\Omega=\Sigma$.

Proof The arguments in the proof of Theorem 1 in [21] are still valid in our current setting and we leave the details to the reader. 


\subsection{Proof of Theorem 8.1 when $K_{\Sigma}$ is hyperbolic and case (S2.3) holds}

Lemma 8.6 Suppose that $K_{\Sigma}$ is generated by a 1-parameter hyperbolic subgroup $\Gamma^{H}$ of $\widetilde{\mathrm{SL}}(2, \mathbb{R})$, and that case $(S 2.3)$ holds. Let $\mathbb{H}_{\theta}^{2}$ be one of the two two-dimensional subgroups of $\widetilde{\mathrm{SL}}(2, \mathbb{R})$ containing $\Gamma^{H}$. Let $\Gamma^{P}, K^{P}$ be the parabolic subgroup of $\mathbb{H}_{\theta}^{2}$ and the right invariant vector field on $\widetilde{\mathrm{SL}}(2, \mathbb{R})$ generated by $\Gamma^{P}$, respectively. Then, $f(\Sigma)$ is a smooth entire Killing $K^{P}$-graph.

Proof We argue in a similar way as in the proof of Lemma 8.4. Consider the foliation $\mathcal{F}=\left\{r_{t}\left(\mathbb{H}_{\theta}^{2}\right) \mid t \in \Gamma_{3}\right\}$ of $\widetilde{\mathrm{SL}}(2, \mathbb{R})$ by right cosets of $\mathbb{H}_{\theta}^{2}$, where $\Gamma_{3}$ is the elliptic 1-parameter subgroup associated to $E_{3}$ given by Eq. (4.7). Recall that the leaves of $\mathcal{F}$ are equidistant surfaces to $\mathbb{H}_{\theta}^{2}$.

Suppose first that the angles that $f(\Sigma)$ makes with $\mathcal{F}$ are bounded away from zero. Then, $f(\Sigma)$ is an entire Killing graph with respect to $K^{P}$ as explained just before Claim 6.6, and so Lemma 8.3 holds in this case. Otherwise, we can apply the Slab Property in $\widetilde{\mathrm{SL}}(2, \mathbb{R})$ (Claim 6.6$)$ to conclude that $f(\Sigma)$ is contained in a smallest slab-type region $S$ between two different leaves $L, L^{\prime}$ of $\mathcal{F}$. As we are in the conditions of case (S2.3), there is an element $a \in \widetilde{\mathrm{SL}}(2, \mathbb{R})-\Gamma^{H}$ such that $l_{a}(f(\Sigma))=f(\Sigma)$. As $f(\Sigma)$ is everywhere tangent to the right invariant vector field $K_{\Sigma}$ and $f(\Sigma)$ is not a two-dimensional subgroup of $\widetilde{\mathrm{SL}}(2, \mathbb{R})$, then there exists $\lambda \in \mathbb{R}-\{0\}$ such that $\left(l_{a}\right)_{*}\left(K_{\Sigma}\right)=\lambda K_{\Sigma}$. Observe that the 1-parameter subgroup of $\widetilde{\mathrm{SL}}(2, \mathbb{R})$ generated by $\left(l_{a}\right)_{*}\left(K_{\Sigma}\right)$ is $a \Gamma_{\Sigma} a^{-1}=$ $\left(l_{a} \circ r_{a^{-1}}\right)\left(\Gamma_{\Sigma}\right)$ (see e.g., the proof of Lemma 4.10), hence this last 1-parameter subgroup must be a reparameterization of $\Gamma_{\Sigma}$. By Lemma 8.7 below, either $a^{2}$ lies in the center $Z$ of $\widetilde{\mathrm{SL}}(2, \mathbb{R})$, or $a$ is the product of an element $h \in \Gamma^{H}$ with an element $b \in Z$. In the first case, $a^{2}=e$ since otherwise the left translation by a large power $a^{2 k}$ of $a^{2}$ sends the topological slab $S$ into another topological slab disjoint from $S$, which contradicts that $f(\Sigma)$ is invariant under the left translation by $a^{2 k}$ and $f(\Sigma)$ is contained in $S$. But $a^{2}=e$ implies $a=e$ (because $Z$ is an infinite cyclic subgroup), which contradicts our hypothesis that $a \in \widetilde{\mathrm{SL}}(2, \mathbb{R})-\Gamma^{H}$. Therefore, $a=b h$ with $h \in \Gamma^{H}$ and $b \in Z$. Observe that $b \neq e$ since $a \notin \Gamma^{H}$. Therefore, a large power $a^{n}=b^{n} h^{n}$ of $a$ sends the topological slab $S$ into another topological slab disjoint from $S$ and we find a contradiction as before. This proves Lemma 8.6.

Lemma 8.7 Suppose $a \in \widetilde{\mathrm{SL}}(2, \mathbb{R})$ satisfies $a \Gamma^{H} a^{-1}=\Gamma^{H}$ for some hyperbolic 1-parameter subgroup $\Gamma^{H}$ of $\widetilde{\mathrm{SL}}(2, \mathbb{R})$. Let $\boldsymbol{P}: \widetilde{\mathrm{SL}}(2, \mathbb{R}) \rightarrow$ $\operatorname{PSL}(2, \mathbb{R})=\widetilde{\operatorname{SL}}(2, \mathbb{R}) / Z$ be the quotient homomorphism where $Z$ is the center of $\widetilde{\mathrm{SL}}(2, \mathbb{R})$. Then $\boldsymbol{P}(a) \in \boldsymbol{P}\left(\Gamma^{H}\right)$ or $a^{2} \in Z$.

Proof We identify $\mathbf{P}\left(\Gamma^{H}\right)$ with the set of hyperbolic translations of the Poincaré disk $\mathbb{D}$ along a geodesic $\gamma$. Since $\mathbf{P}(a) \mathbf{P}\left(\Gamma^{H}\right) \mathbf{P}\left(a^{-1}\right)=\mathbf{P}\left(\Gamma^{H}\right)$, we conclude that $\mathbf{P}(a)$ lies in the normalizer subgroup of $\mathbf{P}\left(\Gamma^{H}\right)$ in $\operatorname{PSL}(2, \mathbb{R})$. In 
this case, it is well-known that $\mathbf{P}(a) \in \mathbf{P}\left(\Gamma^{H}\right)$ or $\mathbf{P}(a)$ is an elliptic isometry of order two around some point in $\gamma$. In the second case, $\mathbf{P}(a)^{2}$ is the identity transformation of $\mathbb{D}$, or equivalently $a^{2}$ lies in the kernel of $\mathbf{P}$ which is $Z$.

\subsection{Case (S2.2) is impossible if $K_{\Sigma}$ is hyperbolic}

The proof that $f(\Sigma)$ cannot be in the conditions of case (S2.2) if $K_{\Sigma}$ is hyperbolic will be an adaptation to the $\widetilde{\mathrm{SL}}(2, \mathbb{R})$ setting of our arguments in Sect. 7.3. In order to do this, we prove first a radius estimate (Lemma 8.9) for compact stable minimal surfaces with boundary contained in some special regions of $\widetilde{\mathrm{SL}}(2, \mathbb{R})$, similar in spirit to Theorem 1.1 in [25]. To start, we next associate to every hyperbolic 1-parameter subgroup $\Gamma^{H}$ of $\widetilde{S L}(2, \mathbb{R})$ a cylindrical-type neighborhood of $\Gamma^{H}$ in $X$, which depends on the left invariant metric and on a number $r>0$. We will call this neighborhood of $\Gamma^{H}$ an infinite box.

Definition 8.8 Let $\Gamma^{H}$ be a hyperbolic 1-parameter subgroup of $\widetilde{\mathrm{SL}}(2, \mathbb{R})$, and let $\gamma \subset \mathbb{H}^{2}(-4)$ be the geodesic with the same extrema as the circle arc $\Pi\left(\Gamma^{H}\right) \subset \mathbb{H}^{2}(-4)$ (recall that $\Pi$ was defined in (4.10)). Note that $\Pi\left(\Gamma^{H}\right)$ is at constant hyperbolic distance $r_{0}$ from $\gamma$, for some $r_{0} \geq 0$. Given $r>r_{0}$, let $\gamma_{r}^{+}, \gamma_{r}^{-} \subset \mathbb{H}^{2}(-4)$ be the two arcs at distance $r$ from $\Pi\left(\Gamma^{H}\right)$ and let $P^{+}(r)=\Pi^{-1}\left(\gamma_{r}^{+}\right), P^{-}(r)=\Pi^{-1}\left(\gamma_{r}^{-}\right)$be the topological planes inside $\widetilde{\mathrm{SL}}(2, \mathbb{R})$ obtained after lifting these arcs through $\Pi$. Let $\mathbb{H}=\mathbb{H}_{\theta}^{2}$ be one of the two two-dimensional subgroups of $\widetilde{\mathrm{SL}}(2, \mathbb{R})$ that contain $\Gamma^{H}$, and call $\mathbb{H} a_{r}^{+}, \mathbb{H} a_{r}^{-}$the right cosets of $\mathbb{H}$ at distance $r$ from $\mathbb{H}$ (here $a_{r}^{+}, a_{r}^{-}$are some elements in $\widetilde{\mathrm{SL}}(2, \mathbb{R}))$. Observe that each of the surfaces $P^{+}(r), P^{-}(r)$ intersects transversely both $\mathbb{H} a_{r}^{+}$and $\mathbb{H} a_{r}^{-}$, along integral curves of the hyperbolic right invariant vector field $K^{H}$ generated by the left action of $\Gamma^{H}$ on $\widetilde{S L}(2, \mathbb{R})$, see Fig. 8.

With these ingredients, we define the infinite box $\mathcal{B}\left(\Gamma^{H}, r\right)$ to be the closure of the component of $\widetilde{\mathrm{SL}}(2, \mathbb{R})-\left[P^{+}(r) \cup P^{-}(r) \cup\left(\mathbb{H} a_{r}^{+}\right) \cup\left(\mathbb{H} a_{r}^{-}\right)\right]$that has portions of all four surfaces in its boundary. Observe that $\Gamma^{H}$ is contained in the interior of $\mathcal{B}\left(\Gamma^{H}, r\right)$.

Lemma 8.9 Consider an infinite box $\mathcal{B}\left(\Gamma^{H}, r\right)$ as before. Then:

(1) $\mathcal{B}\left(\Gamma^{H}, r\right)$ is invariant under the left translations by elements in $\Gamma^{H}$.

(2) Every compact, immersed minimal surface $M \subset X$ whose boundary lies in $\mathcal{B}\left(\Gamma^{H}, r\right)$ satisfies that $M \subset \mathcal{B}\left(\Gamma^{H}, r\right)$.

(3) There exists a constant $R(r)>0$ such that for every compact, stable, immersed minimal surface $h: M \leftrightarrow X$ with $h(\partial M) \subset \mathcal{B}\left(\Gamma^{H}, r\right)$, the intrinsic radius of $M$ is less than $R(r)$.

Proof Item 1 follows from the definitions of the surfaces in the boundary of $\mathcal{B}\left(\Gamma^{H}, r\right)$, all of which are invariant under left translations by elements in $\Gamma^{H}$. 


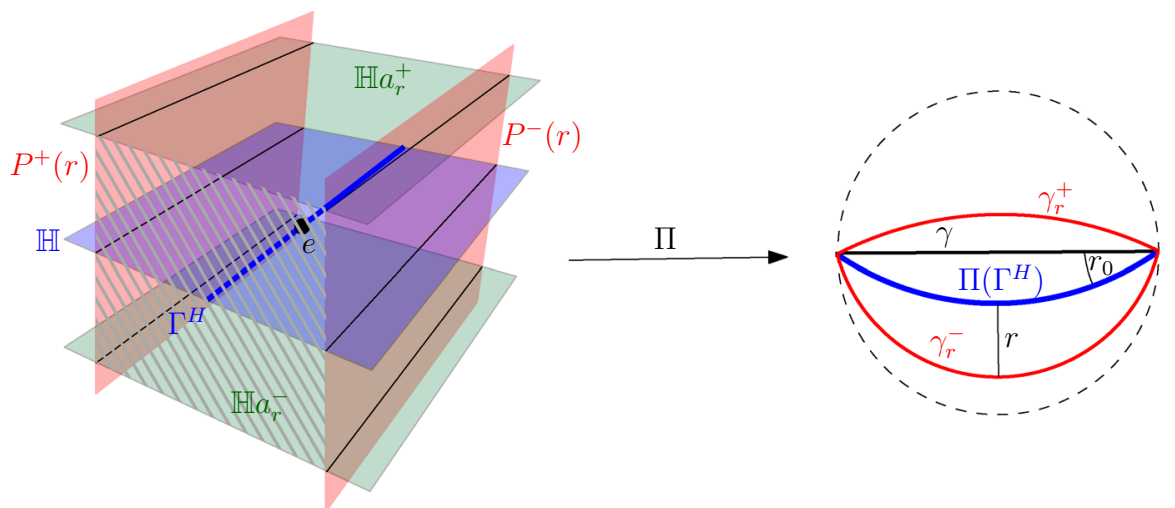

Fig. 8 Schematic representation of an infinite box $\mathcal{B}\left(\Gamma^{H}, r\right)$ in $\widetilde{\mathrm{SL}}(2, \mathbb{R})$, which is the closed, non-compact three-dimensional region invariant under the flow of the hyperbolic 1-parameter subgroup $\Gamma^{H}$ (the blue curve on the left) trapped between the following four topological planes: the two ones represented by red vertical planes are the liftings $P^{+}(r)=\Pi^{-1}\left(\gamma_{r}^{+}\right), P^{-}(r)=$ $\Pi^{-1}\left(\gamma_{r}^{-}\right)$under the canonical projection $\Pi$ of the $\operatorname{arcs} \gamma_{r}^{+}, \gamma_{r}^{-} \subset \mathbb{H}^{2}(-4)$ at distance $r$ of $\Pi\left(\Gamma^{H}\right)$, and the other two represented by green horizontal planes on the left are the two right cosets $\mathbb{H} a_{r}^{+}, \mathbb{H} a_{r}^{-}$of $\mathbb{H}$ at distance $r$ from $\mathbb{H}$, where $\mathbb{H}$ is one of the two two-dimensional subgroups of $\widetilde{\mathrm{SL}}(2, \mathbb{R})$ that contain $\Gamma^{H}$ (represented by a blue horizontal plane in the left) (color figure online)

Item 2 is a direct consequence of the maximum principle and the following facts:

- For every $r^{\prime} \geq r, \mathcal{B}\left(\Gamma^{H}, r^{\prime}\right)$ has piecewise smooth mean convex boundary (this follows from item 4 of Lemma 4.9 and the fact that every right coset $\mathbb{H} a$ is a left translation of some two-dimensional subgroup of $X$, hence minimal), and the inner angles between adjacent smooth surfaces in this boundary are less than $\pi$. Furthermore, $\mathcal{B}\left(\Gamma^{H}, r\right) \subset \mathcal{B}\left(\Gamma^{H}, r^{\prime}\right)$.

- $\bigcup_{r^{\prime}>r} \mathcal{B}\left(\Gamma^{H}, r^{\prime}\right)=X$.

Suppose item 3 of the lemma fails to hold. Then, there exists a sequence of compact, stable, immersed minimal surfaces $h_{n}: M_{n} \rightarrow X$ such that $h_{n}\left(\partial M_{n}\right) \subset \mathcal{B}\left(\Gamma^{H}, r\right)$ and points $p_{n} \in M_{n}$ such that the intrinsic distances from $p_{n}$ to the boundary of $M_{n}$ satisfy $d_{M_{n}}\left(p_{n}, \partial M_{n}\right)>n$ for all $n \in \mathbb{N}$. Next we show that after appropriate left translations and passing to a subsequence, we can take limits of the $h_{n}$. Let $\beta$ be the geodesic in $\mathbb{H}^{2}(-4)$ that passes through $\Pi(e)$ and is orthogonal to $\gamma$. Note that each integral curve in $\widetilde{\mathrm{SL}}(2, \mathbb{R})$ of the hyperbolic right invariant vector field $K^{H}$ generated by $\Gamma^{H}$ intersects the surface $\Pi^{-1}(\beta)$ exactly once; in particular, the integral curve $\Gamma^{H} h_{n}\left(p_{n}\right)=r_{h_{n}\left(p_{n}\right)}\left(\Gamma^{H}\right)$ of $K^{H}$ passing through the point $h_{n}\left(p_{n}\right)$ intersects the compact surface $Y=\Pi^{-1}(\beta) \cap \mathcal{B}\left(\Gamma^{H}, r\right)$ just once (to see this, observe that by the already proven items 1,2 of this lemma, $\mathcal{B}\left(\Gamma^{H}, r\right)$ is invariant under the left action of $\Gamma^{H}$ and $\left.h_{n}\left(M_{n}\right) \subset \mathcal{B}\left(\Gamma^{H}, r\right)\right)$. Hence, after replacing $h_{n}$ by 
a left translation by some element in $\Gamma^{H}$, we will have that $h_{n}\left(p_{n}\right) \in Y$ and $h_{n}\left(M_{n}\right)$ still lies in $\mathcal{B}\left(\Gamma^{H}, r\right)$. As $Y$ is compact, after choosing a subsequence we may assume that $h_{n}\left(p_{n}\right)$ converges to a point $q_{\infty} \in Y$.

By curvature estimates for stable minimal surfaces (Schoen [38], Ros [37]), the immersions $h_{n}$ restricted to the intrinsic balls

$$
B_{M_{n}}\left(p_{n}, n / 2\right)=\left\{x \in M_{n} \mid d_{M_{n}}\left(p_{n}, x\right)<\frac{n}{2}\right\}
$$

have uniformly bounded second fundamental forms. Hence, after choosing a subsequence, we obtain a complete minimal immersion $h_{\infty}: M_{\infty} \rightarrow$ $\mathcal{B}\left(\Gamma^{H}, r\right)$ with bounded second fundamental form that is a limit of the restriction of the $h_{n}$ to compact domains in $M_{n}$, and such that $h_{\infty}\left(p_{\infty}\right)=q_{\infty}$ for some point $p_{\infty} \in M_{\infty}$. Consider the set

$$
\mathcal{M}=\overline{\left\{a h_{\infty}\left(M_{\infty}\right)=l_{a}\left(h_{\infty}\left(M_{\infty}\right)\right) \mid a \in \Gamma^{H}\right\}} \subset \mathcal{B}\left(\Gamma^{H}, r\right),
$$

where for any $A \subset X, \bar{A}$ is the closure of $A$. Since $h_{\infty}\left(M_{\infty}\right)$ has bounded second fundamental form, then given any point $q \in \mathcal{M}$, there exists a compact, embedded minimal disk $D(q) \subset \mathcal{M}$ with $q \in \operatorname{Int}(D(q))$; specifically, the disk $D(q)$ is a limit of embedded geodesic disks of fixed small geodesic radius centered at points $q_{n}$ in some left translate $l_{a_{n}}\left(h_{\infty}\left(M_{\infty}\right)\right)$ such that $\lim _{n \rightarrow \infty} q_{n}=q$, where $a_{n}$ is some element in $\Gamma^{H}$. Since $\mathcal{M} \cap Y$ is a compact set, there exists a largest positive $r^{\prime} \leq r$ such that the set

$$
W=\left[\mathbb{H} a_{r^{\prime}}^{+} \cup \mathbb{H} a_{r^{\prime}}^{-}\right] \cap \mathcal{M} \cap Y
$$

is nonempty. Note that the $\Gamma^{H}$-invariance of $\left[\mathbb{H} a_{r^{\prime}}^{+} \cup \mathbb{H} a_{r^{\prime}}^{-}\right] \cap \mathcal{M}$ implies that $r^{\prime}$ is also the largest positive number such that $\left[\mathbb{H} a_{r^{\prime}}^{+} \cup \mathbb{H} a_{r^{\prime}}^{-}\right] \cap \mathcal{M}$ is nonempty. Let $q \in W$. Then by the maximum principle for minimal surfaces, the set $\mathbb{H} a_{r^{\prime}}^{+} \cup \mathbb{H} a_{r^{\prime}}^{-}$must contain the aforementioned embedded minimal disk $D(q)$. But in this case, $\mathcal{M}$ would have to contain one of the surfaces $\mathbb{H} a_{r^{\prime}}^{+}, \mathbb{H} a_{r^{\prime}}^{-}$ which is false since neither of these surfaces is entirely contained in $\mathcal{B}\left(\Gamma^{H}, r\right)$. This contradiction proves the lemma.

With Lemma 8.9 at hand, we can now rule out case (S2.2).

Proposition 8.10 If $K_{\Sigma}$ is hyperbolic, then case (S2.2) does not occur for $f$.

Proof We will adapt the arguments of Sect. 7.3 to our current setting, focusing on the differences with the case of $X$ being a semidirect product. Suppose that $K_{\Sigma}=K^{H}$ is hyperbolic and that case (S2.2) holds. Recall that $f:(\Sigma, p) \rightarrow$ $(X, e)$ is a complete stable pointed immersion with constant mean curvature $h_{0}(X)$, that is obtained as a limit of pointed immersions $f_{n}:\left(S_{n}, p_{n}\right) \leftrightarrow(X, e)$ 
of spheres $S_{n} \in \mathcal{C}$. Since each sphere $S_{n} \in \mathcal{C}$ is Alexandrov embedded, for each $n \in \mathbb{N}$ there exists a three-dimensional Riemannian manifold $\left(B_{n}, g_{n}\right)$ which is topologically a closed ball, together with a Riemannian submersion $\widetilde{f}_{n}:\left(B_{n}, g_{n}\right) \rightarrow X$ such that $\partial B_{n}=S_{n},\left.\widetilde{f}_{n}\right|_{S_{n}}=f_{n}$ and $\partial B_{n}$ is mean convex. The key step in the proof of Proposition 8.10 will be proving that Lemma 7.5 holds in our situation. Observe that if we admit the validity of Lemma 7.5 in our current setting, then all the arguments in Sect. 7.4 after the proof of Lemma 7.5 remain valid in $\widetilde{S L}(2, \mathbb{R})$ and the proof of Proposition 8.10 will be finished. Therefore, we will only concentrate on the proof of the statement of Lemma 7.5 in our current $\widetilde{S L}(2, \mathbb{R})$-setting.

Consider the hyperbolic 1-parameter subgroup $\Gamma^{H}$ of $\widetilde{\mathrm{SL}}(2, \mathbb{R})$ generated by $K_{\Sigma}$. Since $f(\Sigma)$ is an annulus invariant under the flow of $K^{\Sigma}$, we can find $r_{0}>0$ such that $f(\Sigma)$ is contained in an infinite box $\mathcal{B}\left(\Gamma^{H}, r_{0}\right)$ and $\partial \mathcal{B}\left(\Gamma^{H}, r_{0}\right)$ is at a positive distance from $f(\Sigma)$; in what follows, $\mathcal{B}\left(\Gamma^{H}, r_{0}\right)$ will play the role of the solid cylinder $\mathcal{W}\left(\Gamma, r_{0}\right)$ of the original proof of Lemma 7.5 for metric semidirect products.

By item 3 of Lemma 8.9, there exists some $R=R\left(r_{0}\right)>0$ with the following property:

$(\star)^{\prime}$ If $M$ is any compact, stable immersed minimal surface in $X$ whose boundary $\partial M$ is contained in $\mathcal{B}\left(\Gamma^{H}, r_{0}\right)$, then the intrinsic radius of $M$ is less than $R$.

The role of the horizontal planes in the proof of the semidirect product case of Lemma 7.5 will be now played by the leaves of the foliation $\mathcal{F}^{\prime}=\left\{P_{t}=\right.$ $\left.l_{t}\left(\Pi^{-1}(\beta)\right) \mid t \in \Gamma^{H} \equiv \mathbb{R}\right\}$, where $\beta$ is the geodesic in $\mathbb{H}^{2}(-4)$ that passes through $\Pi(e)$ and is orthogonal to $\gamma$, where both $\gamma$ and $\beta$ were previously defined in the proof of Lemma 8.9. Notice that $\mathcal{F}^{\prime}$ is also the foliation of $X$ whose projection by $\Pi$ to $\mathbb{H}(-4)$ is the foliation of $\mathbb{H}(-4)$ by the set of geodesics orthogonal to $\gamma$, and the leaves of $\mathcal{F}^{\prime}$ are minimal topological planes by Lemma 4.9 .

As $\Sigma$ is simply connected and $f: \Sigma \rightarrow X$ is invariant under the flow of $K_{\Sigma}$, then by the discussion in Remark 4.2 we may parameterize $f$ as in Eq. (7.3) where $\Gamma$ is replaced by $\Gamma^{H}$, and $\alpha=\alpha(s): \mathbb{R} \rightarrow P_{0}$ is an immersed $L$-periodic curve parameterized by arc length in the plane $P_{0}$, so that $f(0,0)=\alpha(0)=e$, $L>0$ being the length of $\alpha(\mathbb{R})$.

Observe that $\mathcal{B}\left(\Gamma^{H}, r_{0}\right)$ is foliated by integral curves of $K^{H}$. As $\mathcal{B}\left(\Gamma^{H}, r_{0}\right) \cap$ $P_{0}$ is compact, there exists the maximum length $\mu_{0}$ of all the arcs of integral curves of $K^{H}$ that are contained in the compact piece of $\mathcal{B}\left(\Gamma^{H}, r_{0}\right)$ that lies in the topological slab of $\widetilde{\mathrm{SL}}(2, \mathbb{R})$ determined by the leaves $P_{-R-2}$ and $P_{R+2}$ of $\mathcal{F}^{\prime}$. Given $s_{0} \in \mathbb{R}$, the definition (7.4) of the compact arc $A\left(s_{0}\right) \subset f(\Sigma)$ of the integral curve of $\Gamma^{H}$ that passes through $\alpha\left(s_{0}\right)$ whose endpoints lie in $P_{-R-2}$ and $P_{R+2}$, remains valid after replacing $\Gamma$ by $\Gamma^{H}$, as well as the property that the length of $A\left(s_{0}\right)$ is at most $\mu_{0}$. 
For each $j \in \mathbb{N}$, consider the compact region $K^{j} \subset \Sigma$ defined by Eq. (7.5). Repeating the arguments in the proof of Lemma 7.5 verbatim, each $K^{j}$ produces compact simply connected domains $K_{n}^{j} \subset S_{n}$ for $n$ large, such that $f_{n}\left(K_{n}^{j}\right) \subset \mathcal{B}\left(\Gamma^{H}, r_{0}\right)$ and the sequence $\left\{\left.f_{n}\right|_{K_{n}^{j}}\right\}_{n}$ converges to $\left.f\right|_{K^{j}}$ uniformly as $n \rightarrow \infty$, and we can also find for each $k \in\{1, \ldots, j\}$ a small compact disk $\mathcal{U}^{k}$ centered at $(k L, 0)$ in $K^{j}$ and related compact disks $\mathcal{U}_{n}^{k} \subset K_{n}^{j}$ such that $\left\{\left.f_{n}\right|_{\mathcal{U}_{n}^{k}}\right\}_{n}$ converges to $\left.f\right|_{\mathcal{U}^{k}}$ uniformly as $n \rightarrow \infty$. Furthermore, the $\mathcal{U}_{n}^{k}$ satisfy that the intrinsic distance between $\mathcal{U}_{n}^{i}$ and $\mathcal{U}_{n}^{k}$ inside $K_{n}^{j}$ is greater than $\delta$ for some $\delta>0$ independent of $n, j, k$. Notice however, that in the present setting, while the plane $P_{0}$ intersects $f(\Sigma)$ transversely along the closed immersed curve $\alpha(\mathbb{R})$, we no longer know that $f_{n}^{-1}\left(P_{0}\right)$ is a simple closed curve (since $P_{0}$ is not a two dimensional subgroup). Instead, for any fixed $j$ and for $n$ sufficiently large, we have that $f_{n}^{-1}\left(P_{0}\right) \cap K_{n}^{j}$ is a connected compact embedded arc which intersects each of the disks $\mathcal{U}_{n}^{k} \subset K_{n}^{j}$, where $k \in\{1, \ldots, j\}$. Let $I$ denote a small compact segment in $P_{0}$ centered at $\alpha(0)=e$ and transversal to $f(\Sigma)$ at this point. The $L$-periodicity of $f(s, t)$ in the variable $s$ and the previous convergence properties allow to find for $n=$ $n(j)$ large enough, $j$ points $q_{n}^{k} \in \mathcal{U}_{n}^{k}$ with $f_{n}\left(q_{n}^{k}\right) \in I$, for every $k=1, \ldots, j$. In particular, property $(\star \star)$ in the proof of Lemma 7.5 holds.

Let $J_{n}^{k}$ be the compact connected arc of $f_{n}^{-1}\left(P_{0}\right) \cap K_{n}^{j}$ whose endpoints are $q_{n}^{1}$ and $q_{n}^{k}$, for each $k \in\{1, \ldots, j\}$. Pulling back via $f_{n}$ the tangent part to $f_{n}\left(S_{n}\right)$ of $K_{\Sigma}$, we obtain a nowhere zero vector field $W_{n}$ on $K_{n}^{j}$ for $n$ large enough. Letting the arc $J_{n}^{k}$ flow under $W_{n}$ between the sets $f_{n}^{-1}\left(P_{-R-1}\right)$ and $f_{n}^{-1}\left(P_{R+1}\right)$ we obtain a compact disk $D_{n}^{k} \subset K_{n}^{j}$ which is foliated by compact arcs of integral curves of $W_{n}$, each of which joins points in $\left(\partial D_{n}^{k}\right) \cap$ $f_{n}^{-1}\left(P_{-R-1}\right)$ and $\left(\partial D_{n}^{k}\right) \cap f_{n}^{-1}\left(P_{R+1}\right)$, for $n$ large. Then, the Jordan curve $\gamma_{n}^{k}:=\partial D_{n}^{k}$, can be decomposed as $\gamma_{n}^{k}=A_{1}^{n} \cup A_{2}^{n} \cup B_{1}^{n} \cup B_{2}^{n}$, where properties (T1), (T2) hold after replacing the planes $\mathbb{R}^{2} \rtimes_{A}\{ \pm(R+1)\}$ by the topological planes $P_{ \pm(R+1)}$.

We next solve for $k \in\{1, \ldots, j\}$ fixed (and $n$ large enough) the Plateau problem with boundary $\gamma_{n}^{k}$ in $\left(B_{n}, g_{n}\right)$, finding a compact stable embedded minimal disk $M_{n}^{k} \subset B_{n}$ of least area, with $\partial M_{n}^{k}=\gamma_{n}^{k}$. Furthermore, $M_{n}^{k}$ is an immersion transverse to $\partial B_{n}$ at the interior points of $A_{1}^{n}$ and $A_{2}^{n}$. Let $\Lambda_{n}$ be the embedded surface $\left\{q \in B_{n} \mid \widetilde{f}_{n}(q) \in P_{0}\right\}$ and $\beta_{n}^{k}:=M_{n}^{k} \cap \Lambda_{n}$. Following the same reasoning as in the proof of Lemma 7.5 we deduce that after a small perturbation of $\Lambda_{n}$ in $B_{n}$ that fixes its boundary, we may assume that $\beta_{n}^{k}$ contains a compact arc joining $q_{n}^{1}$ with $q_{n}^{k}$ along which $M_{n}^{k}$ intersects $\Lambda_{n}$ transversally. Equations (7.6), (7.7), (7.8) and (7.9) hold in our current situation with the only changes of $\mathbb{R}^{2} \rtimes_{A}\{ \pm(R+1)\}$ by $P_{ \pm(R+1)}, \mathbb{R}^{2} \rtimes_{A}\{0\}$ by $P_{0}$ and Property $(\star)$ by Property $(\star)^{\prime}$. Finally, the arguments in the last five 
paragraphs of the proof Lemma 7.5 remain valid without changes, by noticing that in our $\widetilde{\mathrm{SL}}(2, \mathbb{R})$-setting, the mean curvature $h_{0}(X)$ of $f$ is positive as explained just before the statement of Theorem 8.1. This finishes the sketch of proof of Lemma 7.5 when $X$ is isomorphic to $\widetilde{\mathrm{SL}}(2, \mathbb{R})$ and $K_{\Sigma}$ is hyperbolic, and also finishes the proof of Proposition 8.10.

\subsection{Case (S2.1) is impossible if $K_{\Sigma}$ is hyperbolic}

Proposition 8.11 If $K_{\Sigma}$ is hyperbolic, then $f(\Sigma)$ cannot be in the conditions of case (S2.1).

Proof Again the proof of this proposition is basically an adaptation of the contents of Sect. 7.4 to the setting of $\widetilde{\mathrm{SL}}(2, \mathbb{R})$. As we did when proving Proposition 8.10, we will only focus on the differences with the case of $X$ being a semidirect product, and we will also use part of the notation established in the proof of Proposition 8.10.

Suppose that case (S2.1) holds and $K_{\Sigma}$ is hyperbolic, with associated 1-parameter subgroup $\Gamma^{H}: \mathbb{R} \rightarrow \widetilde{\mathrm{SL}}(2, \mathbb{R})$. We use the same notation $f:(\Sigma, p) \leftrightarrow(X, e), f_{n}:\left(S_{n}, p_{n}\right) \rightarrow(X, e)$ and $\widetilde{f}_{n}: B_{n} \rightarrow X$ as in the first paragraph of the proof of Proposition 8.10. The desired contradiction will come from application of a flux argument (Theorem 9.1) that we explain next.

Let $\mathbb{H}=\mathbb{H}_{\theta}^{2}$ be one of the two two-dimensional subgroups of $\widetilde{\mathrm{SL}}(2, \mathbb{R})$ that contain $\Gamma^{H}$. Associated to $\mathbb{H}$ and to $\Gamma^{H}$, we have the infinite boxes $\mathcal{B}\left(\Gamma^{H}, r\right)$ for all $r>0$. Since $f(\Sigma)$ is an annulus invariant under the flow of $K_{\Sigma}$, we can find $r_{0}>0$ such that $f(\Sigma)$ is contained in $\mathcal{B}\left(\Gamma^{H}, r_{0}\right)$ and $\partial \mathcal{B}\left(\Gamma^{H}, r_{0}\right)$ is at a positive distance from $f(\Sigma)$. Consider the related foliation $\mathcal{F}^{\prime}=\left\{P_{t} \mid t \in \Gamma^{H} \equiv \mathbb{R}\right\}$ by topological minimal planes defined in the proof of Proposition 8.10.

For $n$ sufficiently large, consider the simple closed curve component $\alpha_{n}$ of $f_{n}^{-1}\left(P_{0}\right) \subset S_{n}$ containing $p_{n}$ and let $K_{\Sigma}^{n}$ be the Killing vector field on $B_{n}$ obtained by pulling-back $K_{\Sigma}$ through $\widetilde{f}_{n}$. As we are assuming that case (S2.1) holds, $\Sigma$ is an annulus and $f(\Sigma)$ is the immersed annulus obtained by letting the immersed closed curve $f(\Sigma) \cap P_{0}$ flow under $K_{\Sigma}$. Let $\alpha_{\infty}=f^{-1}\left(P_{0}\right)$, which is a simple closed curve in $\Sigma$ such that the sequence $\left\{f_{n} \mid \alpha_{n}\right\}_{n}$ converges uniformly to $\left.f\right|_{\alpha_{\infty}}$.

As $\mathcal{F}^{\prime}$ is a foliation by minimal planes, then the maximum principle implies that the smooth, embedded least-area disk $M(n)$ in the abstract Riemannian 3-ball $B_{n}$ obtained by solving the Plateau problem in $B_{n}$ with boundary $\alpha_{n}$, satisfies that $\widetilde{f}_{n}$ restricts to $M(n)$ producing an immersion of $M(n)$ into $P_{0}$. By the convergence of the sequence $\left\{\left.f_{n}\right|_{\alpha_{n}}\right\}_{n}$, it follows that the areas of the minimal disks $\widetilde{f}_{n}(M(n))$ are uniformly bounded. Furthermore, as the mean curvatures of the $S_{n}$ are positive, then $M(n)$ is transverse to $\partial B_{n}$ along $\alpha_{n}$. 
The same argument as in the third paragraph after the proof of Lemma 7.6 proves that there exists an $\eta \in(0, \pi / 4)$ such that the angle that $M(n)$ makes with $S_{n}$ along $\alpha_{n}$ is greater than $\eta$ for all $n \in \mathbb{N}$ large enough, after replacement of the intrinsic radius estimate Theorem 1.1 in [25] by item 3 of Lemma 8.9, changing $\Gamma$ by $\Gamma^{H}$, and noticing that $h_{0}(X) \geq H(X)>0$.

The next step consists of proving that if $N_{M(n)}$ stands for the unit normal field to $M(n)$ in $\left(B_{n}, g_{n}\right)$, then for $n$ sufficiently large the function $J_{n}=$ $g_{n}\left(N_{M(n)}, K_{\Sigma}^{n}\right): M(n) \rightarrow \mathbb{R}$ has no zeros along $\alpha_{n}$. The proof of the same property in Sect. 7.4 does not work now, since it uses Eq. (4.5) which is only valid in a semidirect product. Instead, we will argue in $\widetilde{\mathrm{SL}}(2, \mathbb{R})$ as follows. Observe that

(W) $K_{\Sigma}$ is everywhere transversal to $P_{0}$.

Now, if $J_{n}$ vanishes at some point $q_{n} \in \alpha_{n}$, then $K_{\Sigma}^{n}\left(q_{n}\right)$ is tangent to $M(n)$ at $q_{n}$. Applying the differential of $\tilde{f}_{n}$ at $q_{n}$, we deduce that $K_{\Sigma}\left(f_{n}\left(q_{n}\right)\right)$ is tangent to $P_{0}$ at $f_{n}\left(q_{n}\right)$, which contradicts (W). Therefore, $J_{n}$ has no zeros along $\alpha_{n}$ for all $n \in \mathbb{N}$.

Once we know that $J_{n}$ has no zeros along $\alpha_{n}$ for all $n$, we can follow the arguments in Sect. 7.4 with the following changes (we are using the same notation as in Sect. 7.4):

- Replace $\Gamma$ by $\Gamma^{H}$ in Eqs. (7.13) and (7.14). In this way, we can obtain a submersion $T_{\infty}: \overline{\mathbb{D}} \times \mathbb{R} \rightarrow X$.

- Replace $\mathbb{R}^{2} \rtimes_{A}\{t\}$ in Eq. (7.15) by the horocylinder $\Pi^{-1}\left(c_{t}\right)$ where $c_{t}$ is the horocircle in $\mathbb{H}^{2}(-4)$ whose end point at $\partial_{\infty} \mathbb{H}^{2}$ is $\theta$ defined by $\mathbb{H}=\mathbb{H}_{\theta}^{2}$, such that $c_{t}$ passes through $\Pi(t), t \in \Gamma^{H}$. That is, define $D_{t}=$ $T_{\infty}^{-1}\left(\Pi^{-1}\left(c_{t}\right)\right)$, and then define the foliation $\mathcal{F}$ as in Eq. (7.15).

- Define $K_{1}$ as the nowhere zero, smooth vector field on $\overline{\mathbb{D}} \times \mathbb{R}$ obtained after pulling $K_{\Sigma}$ by the submersion $T_{\infty}$. Observe that each of the integral curves of $K_{1}$ in $\overline{\mathbb{D}} \times \mathbb{R}$ intersects $D_{0}$ in a single point.

- Define $K_{2}$ as the nowhere zero vector field on $W:=D_{0} \times \mathbb{R}$ which is the pullback by $T_{\infty}$ of a parabolic right invariant vector field $V$ on $X$ associated to $\mathbb{H}$ ( $V$ is defined up to a multiplicative nonzero constant). Observe that $K_{2}$ is tangent to the foliation $\mathcal{F}$ and that once we endow $W$ with the pulled-back metric by $T_{\infty}, K_{2}$ is a Killing field. We claim that $K_{2}$ is bounded on one of the two ends of $W$ : to do this, first note that to prove this boundedness property, it suffices to assume that $X$ has isometry group of dimension four (as every left invariant metric on $\widetilde{\mathrm{SL}}(2, \mathbb{R})$ is quasi-isometric to any fixed left invariant metric on $\widetilde{\mathrm{SL}}(2, \mathbb{R})$ ). Also notice that $\Pi^{-1}\left(c_{0}\right) \cap \mathbb{H}$ is the 1-parameter parabolic subgroup of $\mathbb{H}$, and that the restriction of $V$ to $\Pi^{-1}\left(A_{0}\right) \cap \mathbb{H}$ is bounded, where $A_{0}$ is the open horodisk in $\mathbb{H}^{2}$ bounded by $c_{0}$ (in fact, if we identify $\mathbb{H}$ with the semidirect product $\mathbb{R} \rtimes_{(1)} \mathbb{R}=\{(x, y) \mid x, y \in \mathbb{R}\}$ as explained after (8.1), then $V$ 
identifies with $\partial_{x}$ up to a multiplicative constant, $\Pi^{-1}\left(A_{0}\right) \cap \mathbb{H}$ is the open halfspace $\mathbb{R} \rtimes_{(1)}(0, \infty)$ and $\partial_{x}$ is bounded in $\left.\mathbb{R} \rtimes_{(1)}(0, \infty)\right)$. As the right translations by elements in the elliptic subgroup of $\widetilde{S L}(2, \mathbb{R})$ generated by the vector $\left(E_{3}\right)_{e}$ given by Eq. (4.7) are isometries of any $\mathbb{E}(\kappa, \tau)$-metric in $\widetilde{\mathrm{SL}}(2, \mathbb{R})$ and $V$ is right invariant, we conclude that $V$ is bounded in $\Pi^{-1}\left(A_{0}\right)$. Therefore, our claim follows.

The above changes allow us to apply Theorem 9.1 to find the same contradiction with CMC flux as in Sect. 7.4. This contradiction finishes the proof of Proposition 8.11.

Open Access This article is licensed under a Creative Commons Attribution 4.0 International License, which permits use, sharing, adaptation, distribution and reproduction in any medium or format, as long as you give appropriate credit to the original author(s) and the source, provide a link to the Creative Commons licence, and indicate if changes were made. The images or other third party material in this article are included in the article's Creative Commons licence, unless indicated otherwise in a credit line to the material. If material is not included in the article's Creative Commons licence and your intended use is not permitted by statutory regulation or exceeds the permitted use, you will need to obtain permission directly from the copyright holder. To view a copy of this licence, visit http://creativecommons.org/licenses/by/4.0/.

\section{Appendix: A nonvanishing result for the CMC flux}

Let $W$ be a Riemannian $(n+1)$-manifold and $M \subset W$ a two-sided $n$ hypersurface with constant mean curvature $H \in \mathbb{R}$ (throughout this appendix we define the mean curvature of $M$ to be the trace of its second fundamental form divided by $n$ ). The CMC flux of $M$ associated to a Killing vector field $K$ on $W$ and to a piecewise smooth $(n-1)$-chain $\alpha$ in $M$ which is the boundary of a piecewise smooth two-sided $n$-chain $\beta$ in $W$, is the real number

$$
\operatorname{Flux}(M, \alpha, K)=\int_{\alpha}\langle K, \eta\rangle+n H \int_{\beta}\langle K, N\rangle,
$$

where $\eta$ is a unit conormal vector to $M$ along $\alpha$ and $N$ is a unit normal vector field along $\beta$. In order for (9.1) not to depend on orientation choices, certain compatibility between $H, \eta$ and $N$ must be satisfied, see a more precise definition in Definition 9.2. A key property of $\operatorname{Flux}(M, \alpha, K)$ is that it does not depend on the homology class of $\alpha$ in $M$.

The goal of this section is to prove the following general result, in which the CMC flux of an $n$-hypersurface $M=\partial \Sigma \times \mathbb{R}$ with respect to a certain Killing vector field $K=K_{1}$ is nonzero. Theorem 9.1 has been used in Sects. 7.4 and 8.5. 
Theorem 9.1 Let $\Sigma$ be a smooth, compact oriented n-manifold with nonempty boundary, $n \leq 6$. Let $W$ be $\Sigma \times \mathbb{R}$ equipped with a Riemannian metric such that:

(1) The boundary $\partial \Sigma \times \mathbb{R}$ of $W$ is mean convex, with constant mean curvature $h_{0} \geq 0$.

(2) There exists a nowhere zero Killing field $K_{1}$ of $W$ arising from a proper action $^{4}$ of an $\mathbb{R}$-subgroup of the isometry group of $W$.

Suppose that there exists a Killing field $K_{2}$ in $W$ which is tangent to the foliation $\{\Sigma \times\{s\} \mid s \in \mathbb{R}\}$, bounded on either $\Sigma \times[0, \infty)$ or on $\Sigma \times(-\infty, 0]$ and such that every integral curve of $K_{2}$ that intersects $\operatorname{Int}(W)$ is a compact arc with boundary in $\partial W$. Then:

$$
\operatorname{Flux}\left(\partial \Sigma \times \mathbb{R}, \partial \Sigma \times\{0\}, K_{1}\right) \neq 0
$$

Definition 9.2 (CMC flux). Suppose $M$ is a hypersurface with constant mean curvature $H \in \mathbb{R}$ in an oriented Riemannian $(n+1)$-manifold $X$, and let $K$ be a Killing field on $X$. For each $[\alpha] \in H_{n-1}(M)$ in the kernel of the induced inclusion homomorphism $i_{*}: H_{n-1}(M) \rightarrow H_{n-1}(X)$, consider two homologous piecewise smooth $(n-1)$-cycles $\alpha, \alpha_{1} \subset M$ representing $[\alpha]$ and let $\beta, \beta_{1}$ be piecewise smooth $n$-chains in $X$ with boundaries $\partial \beta=\alpha$, $\partial \beta_{1}=\alpha_{1}$ (which exist since $[\alpha] \in \operatorname{ker}\left(i_{*}\right)$ ). We assume that if $M\left(\alpha, \alpha_{1}\right)$ is the $n$-chain in $M$ whose boundary is $\alpha_{1}-\alpha$, then $\beta-\beta_{1}-M\left(\alpha, \alpha_{1}\right)$ is the boundary of an $(n+1)$-chain $\Omega$ in $X$. Applying the Divergence Theorem to $K$ in $\Omega$, we obtain

$$
0=-\int_{\beta}\langle K, N\rangle+\int_{\beta_{1}}\left\langle K, N_{1}\right\rangle-\int_{M\left(\alpha, \alpha_{1}\right)}\left\langle K, N^{M}\right\rangle,
$$

where $-N, N_{1}$ are unit normal vector fields to $\beta, \beta_{1}$ (defined almost everywhere) pointing outward $\Omega$, and $N^{M}$ is the unit normal vector field to $M$ pointing inward $\Omega$ (see Fig. 9). By the Gauss equation, the divergence in $M$ of the tangential part $K^{T}$ of $K$ is given by $\operatorname{div}^{M}\left(K^{T}\right)=n H\left\langle K, N^{M}\right\rangle$, where $H$ is the mean curvature of $M$ with respect to $N^{M}$. Applying the Divergence Theorem in $M\left(\alpha, \alpha_{1}\right)$,

$$
n H \int_{M\left(\alpha, \alpha_{1}\right)}\left\langle K, N^{M}\right\rangle=\int_{\alpha}\langle K, \eta\rangle-\int_{\alpha_{1}}\left\langle K, \eta_{1}\right\rangle,
$$

\footnotetext{
4 This means that if we denote by $\left\{\phi_{s} \mid s \in \mathbb{R}\right\}$ the 1-parameter subgroup of isometries of $W$ corresponding to the flow of the Killing field $K_{1}$, then for each $x \in W$ the map $s \in \mathbb{R} \mapsto \phi_{S}(x)$ is proper.
} 
Fig. 9 Interpretation of the

CMC flux homological invariant

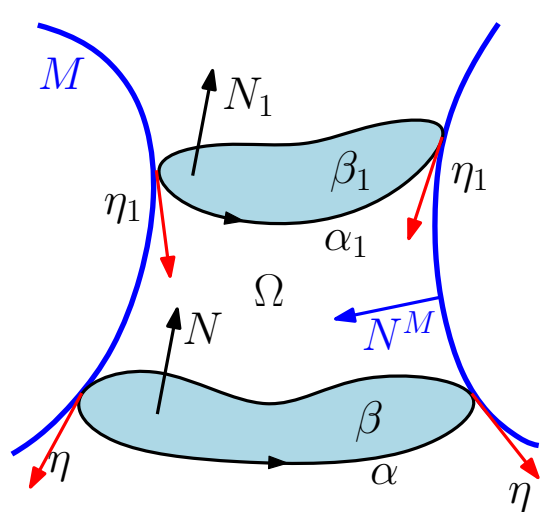

where $\eta,-\eta_{1}$ are the unit conormal vector fields to $M$ along $\alpha, \alpha_{1}$ pointing outward $M\left(\alpha, \alpha_{1}\right)$, which are defined almost everywhere on $\alpha, \alpha_{1}$. Equations (9.2), (9.3) show that the real number

$$
\operatorname{Flux}(M, \alpha, K)=\int_{\alpha}\langle K, \eta\rangle+n H \int_{\beta}\langle K, N\rangle
$$

is independent of the choice of the representative $\alpha$ in a given homology class $[\alpha] \in \operatorname{ker}\left(i_{*}\right) \subset H_{n-1}(M)$ and of the $n$-chain $\beta$ as above. This number is usually called the $C M C$ flux of $M$ along $[\alpha]$ associated to $K$. Observe that the mean curvature of $M$ is computed with respect to the unit normal vector that, along $\alpha$, points to the same closed component of $M \cup \beta$ as $N$, and that $\langle N, \eta\rangle \leq 0$ along $\alpha$. The above argument shows the homological invariance of the CMC flux; we refer the reader to the papers $[15,19,20,22]$ for applications of this CMC flux.

Proof of Theorem 9.1 Without loss of generality, we will assume that the Killing field $K_{2}$ is bounded on the end $\Sigma \times(-\infty, 0]$ of $W$. Let $\left\{\phi_{s} \mid s \in \mathbb{R}\right\}$ be the 1-parameter subgroup of isometries of $X$ corresponding to the flow of the Killing field $K_{1}$. Since the action $(s, x) \in \mathbb{R} \times W \mapsto \phi_{s}(x)$ is proper, then after possibly changing $K_{1}$ by $-K_{1}$, we may suppose that $\phi_{S}(\Sigma \times\{0\})$ lies eventually in the upper end $\Sigma \times[0, \infty)$ of $W$ as $s \rightarrow+\infty$. Consider the quotient map $\Pi: W \rightarrow W / G=\widehat{W}$, where $G=\left\{\phi_{s} \mid s \in 2 \pi \mathbb{Z}\right\}$ (note that the discrete group of isometries $G$ acts proper discontinuously on $W$ ). We will denote by $\mathbb{S}^{1}$ the related 1-parameter subgroup of isometries of $\widehat{W}$.

Let $\widehat{\Gamma}$ be an area-minimizing oriented $(n-1)$-dimensional hypersurface in $\partial \widehat{W}$ that represents $[\partial \Sigma] \in H_{n-1}(\partial \widehat{W})(\widehat{\Gamma}$ is an area-minimizing integral current, which is smooth in this dimension and it exists since $\partial \widehat{W}$ is a closed Riemannian manifold). By cut-and-paste type arguments and the maximum 
principle for minimal hypersurfaces, any two distinct solutions to this areaminimization problem in $\partial \widehat{W}$ are disjoint or equal. It follows that $\partial \widehat{W}$ is foliated by the area-minimizing oriented $(n-1)$-dimensional submanifolds $\phi_{S}(\widehat{\Gamma})$, $\phi_{s} \in \mathbb{S}^{1}$. For homological reasons, $\widehat{\Gamma}$ has a lift through $\Pi$ which is a compact $(n-1)$-dimensional hypersurface $\Gamma$ in $\partial W$ that is area-minimizing in $\partial W$, and when considered to lie in $W$, it is the boundary of an integral $n$-chain. By the properness of the $\mathbb{R}$-action giving rise to $K_{1}$ and the compactness of $\Gamma$, we deduce that $K_{1}$ cannot be everywhere tangent to $\Gamma$. On the other hand, since for $s \neq 0$ it holds $\phi_{s}(\Gamma) \cap \Gamma=\varnothing$, then the Jacobi function $J_{\Gamma}=\left\langle K_{1}, N_{\Gamma}\right\rangle: \Gamma \rightarrow \mathbb{R}$, is nonnegative on $\Gamma$ (after choosing an appropriate orientation of $\Gamma$, or equivalently a unit normal $N_{\Gamma}$ along $\Gamma$, i.e., $N_{\Gamma}$ is tangent to $\partial W$, orthogonal to $\Gamma$ and unitary); note that $J_{\Gamma}$ is positive at the points of $\Gamma$ where $K_{1}$ is not tangent to $\Gamma$. The maximum principle (see e.g., [29, Assertion 2.2]) implies that $J_{\Gamma}>0$ on $\Gamma$; in particular, $K_{1}$ is transverse to $\Gamma$ and each integral curve of $K_{1}$ intersects $\Gamma$ in a single point.

Let $\Lambda \subset W$ be an area-minimizing hypersurface with boundary $\Gamma$, which in this dimension is smooth by standard interior and boundary regularity results for minimizing integral varifolds; see for example, Hardt and Simon [14] for boundary regularity results and also see $[31,33]$ for related barrier arguments. By the mean curvature comparison principle, $\Lambda$ is never tangent to $\partial W$ along $\Gamma$. Since $J_{\Gamma}>0$ by the previous paragraph, then we can choose a unit normal vector $N_{\Lambda}$ to $\Lambda$ such that $\left\langle\left.\left(N_{\Lambda}\right)\right|_{\partial \Gamma}, N_{\Gamma}\right\rangle$ is positive along $\Gamma$. As $K_{1}$ is tangent to $\partial W,\left.\left(N_{\Lambda}\right)\right|_{\Gamma}$ is orthogonal to $\Gamma$ and both $\left\langle K_{1}, N_{\Gamma}\right\rangle,\left\langle\left.\left(N_{\Lambda}\right)\right|_{\partial \Gamma}, N_{\Gamma}\right\rangle$ are positive along $\Gamma$, then we conclude that $\left\langle K_{1}, N_{\Lambda}\right\rangle>0$ along $\Gamma$. As $\Lambda$ is stable and $\left\langle K_{1}, N_{\Lambda}\right\rangle>0$ along $\partial \Lambda$, then $\left\langle K_{1}, N_{\Lambda}\right\rangle$ is positive on $\Lambda$ and so, $\Lambda$ intersects each of the integral curves of $K_{1}$ transversely in a single point. Therefore, the set $\mathcal{F}=\left\{\phi_{s}(\Lambda) \mid s \in \mathbb{R}\right\}$ is a smooth minimal foliation of $W$, where each leaf $\phi_{S}(\Lambda)$ of this foliation intersects every integral curve of $K_{1}$ transversely in a single point.

We will now deform the minimal surface $\Lambda$ by a 1-parameter family of surfaces with constant mean curvature and the same boundary as $\Lambda$. By the "blowing a bubble" technique described in [19], for $\delta>0$ sufficiently small there exists a unique 1-parameter family of hypersurfaces $t \in[0, \delta) \mapsto \Lambda(t) \subset$ $W$ depending smoothly on $t$ such that for all $t \in[0, \delta)$ :

(X1) $\Lambda(t)$ is the graph of a smooth function $f_{t}: \Lambda \rightarrow \mathbb{R}$ (in the direction of $\left.K_{1}\right)$, with $\Lambda(0)=\Lambda$ and $f_{0}=0$.

(X2) $\Lambda(t)$ has constant mean curvature $-t$ with respect to the unit normal vector field $N_{\Lambda(t)}$ to $\Lambda(t)$ that satisfies $\left\langle K_{1}, N_{\Lambda(t)}\right\rangle>0$.

(X3) $f_{t}=0$ on $\partial \Lambda$.

(X4) $f_{t}(x)>f_{t^{\prime}}(x)$ whenever $t>t^{\prime}$ and $x \in \operatorname{Int}(\Lambda)$.

We now study the maximal half-open interval $\left[0, T_{0}\right)$ of $t$-values in which the family $\Lambda(t)$ can be defined (here $\left.T_{0} \in(0, \infty]\right)$. 
First consider the case in which the constant mean curvature $h_{0}$ of $\partial W$ satisfies $h_{0}<T_{0}$. Then, there exists a hypersurface $\Lambda\left(t_{1}\right) \subset X$ with constant mean curvature $-t_{1}$, which is the graph of a function $f_{t_{1}}: \Lambda \rightarrow \mathbb{R}$ such that $f_{t_{1}}=0$ on $\partial \Lambda$ and $t_{1}>h_{0}$. Since $\partial \Lambda(0)=\partial \Lambda\left(t_{1}\right)$, then the CMC flux of $\Lambda\left(t_{1}\right)$ along $\left[\partial \Lambda\left(t_{1}\right)\right]$ associated to $K_{1}$ is given by

$$
\operatorname{Flux}\left(\Lambda\left(t_{1}\right), \partial \Lambda\left(t_{1}\right), K_{1}\right)=\int_{\partial \Lambda(0)}\left\langle K_{1}, \eta_{\Lambda\left(t_{1}\right)}\right\rangle+n t_{1} \int_{\Lambda(0)}\left\langle K_{1}, N_{\Lambda(0)}\right\rangle,
$$

where $\eta_{\Lambda\left(t_{1}\right)}$ is the outward pointing unit conormal vector field to $\Lambda\left(t_{1}\right)$ along $\partial \Lambda\left(t_{1}\right)$ and $N_{\Lambda(0)}$ is the unit normal vector field to $\Lambda(0)$ pointing towards the compact region of $W$ bounded by $\Lambda(0)$ and $\Lambda\left(t_{1}\right)$. Note that

$$
\operatorname{Flux}\left(\Lambda\left(t_{1}\right), \partial \Lambda\left(t_{1}\right), K_{1}\right)=0
$$

by the homological invariance of the CMC flux $\left(\partial \Lambda\left(t_{1}\right)\right.$ is homologically trivial in $\left.\Lambda\left(t_{1}\right)\right)$. On the other hand, along $\partial \Lambda(0)$ the inequality

$$
\left\langle K_{1}, \eta_{(\partial W)^{+}}\right\rangle \leq\left\langle K_{1}, \eta_{\Lambda\left(t_{1}\right)}\right\rangle
$$

holds, where $\eta_{(\partial W)^{+}}$denotes the outward pointing unit conormal field to the portion $(\partial W)^{+}$of $\partial W$ which lies above $\partial \Lambda(0)$. This last inequality together with $h_{0}<t_{1}$ imply that

$$
\begin{aligned}
\operatorname{Flux}\left((\partial W)^{+}, \partial \Lambda(0), K_{1}\right) & =\int_{\partial \Lambda(0)}\left\langle K_{1}, \eta_{(\partial W)^{+}}\right\rangle+n h_{0} \int_{\Lambda(0)}\left\langle K_{1}, N_{\Lambda(0)}\right\rangle \\
& <\int_{\partial \Lambda(0)}\left\langle K_{1}, \eta_{(\partial W)^{+}}\right\rangle+n t_{1} \int_{\Lambda(0)}\left\langle K_{1}, N_{\Lambda(0)}\right\rangle \\
& \stackrel{(9.5)}{\leq} \operatorname{Flux}\left(\Lambda\left(t_{1}\right), \partial \Lambda\left(t_{1}\right), K_{1}\right)=0 .
\end{aligned}
$$

Finally, the homological invariance of the CMC flux gives

$$
\begin{aligned}
\text { Flux }\left(\partial W, \partial \Sigma \times\{0\}, K_{1}\right) & =\text { Flux }\left((\partial W)^{+}, \partial \Sigma \times\{0\}, K_{1}\right) \\
& =\operatorname{Flux}\left((\partial W)^{+}, \partial \Lambda(0), K_{1}\right)<0,
\end{aligned}
$$

which completes the proof of the theorem in the case $h_{0}<T_{0}$.

Assume in the sequel that $T_{0} \leq h_{0}$ and we will find a contradiction, which will finish the proof of the theorem. We first prove the following property.

Assertion 9.3 $\cup_{t \in\left[0, T_{0}\right)} \Lambda(t)$ is not contained in any compact region of $W$. In particular, for every $n \in \mathbb{N}$ there exists a $t(n) \in\left[0, T_{0}\right)$ such that $\Lambda(t)$ contains points at distance at least $n$ from $\Lambda(0)$ whenever $t \geq t(n)$. 
Proof of the assertion. Suppose the assertion does not hold. Then, the constant mean curvature hypersurfaces $\left\{\Lambda(t) \mid t \in\left[0, T_{0}\right)\right\}$ have uniform height estimates (as $K_{1}$-graphs). By the "blowing a bubble" technique in [19], these height estimates imply that the $\left\{\Lambda(t) \mid t \in\left[0, T_{0}\right)\right\}$ satisfy uniform curvature estimates, i.e., they have uniform bounds on the length of their second fundamental forms; these uniform curvature estimates can also be seen to hold since $\Lambda(t)$ minimizes the functional Volume ${ }_{n}+n t$ Volume $_{n+1}$ with respect to compact hypersurfaces in $W$ with boundary $\partial \Lambda$ that are homologous to $\Lambda$ relative to $\partial \Lambda$, where Volume ${ }_{k}$ is the volume of any $k$-chain. From here, standard compactness results show that the top boundary component of $\cup_{t \in\left[0, T_{0}\right)} \Lambda(t)$ is a hypersurface $\Lambda\left(T_{0}\right)$ with constant mean curvature $-T_{0}$.

Note that with respect to the unit normal vector field $N_{\Lambda\left(T_{0}\right)}$ to $\Lambda\left(T_{0}\right)$ that is the limit of the $N_{\Lambda(t)}$ as $t \nearrow T_{0}$, the function $\left\langle K_{1}, N_{\Lambda\left(T_{0}\right)}\right\rangle$ is nonnegative, and it is positive at some point by previous arguments. Since $\left\langle K_{1}, N_{\Lambda\left(T_{0}\right)}\right\rangle$ is a Jacobi function on $\Lambda\left(T_{0}\right)$, then the maximum principle (see e.g., [29, Assertion 2.2]) implies that $\left\langle K_{1}, N_{\Lambda\left(T_{0}\right)}\right\rangle>0$ at $\operatorname{Int}\left(\Lambda\left(T_{0}\right)\right)$. In particular, $\Lambda\left(T_{0}\right)$ is the $K_{1^{-}}$ graph of a continuous function $f_{T_{0}}: \Lambda \rightarrow \mathbb{R}$ that is smooth at $\operatorname{Int}\left(\Lambda\left(T_{0}\right)\right)$. As $\Lambda\left(T_{0}\right)$ is not contained in $\partial W$, then the mean curvature comparison principle and the Hopf boundary maximum principle imply that the gradient of $f_{T_{0}}$ is bounded as we approach $\partial \Lambda\left(T_{0}\right)$; hence, $\left\langle K_{1}, N_{\Lambda\left(T_{0}\right)}\right\rangle$ is positive along $\partial \Lambda\left(T_{0}\right)$. It follows from the maximum principle that $\left\langle K_{1}, N_{\Lambda\left(T_{0}\right)}\right\rangle$ is positive in $\Lambda\left(T_{0}\right)$ and thus, $f_{T_{0}}$ is smooth in $\Lambda$. Therefore, items (X1)-(X4) hold for $t=T_{0}$. In this situation, the strict stability of $\Lambda\left(T_{0}\right)$ implies that the family of hypersurfaces $\left\{\Lambda(t) \mid t \in\left[0, T_{0}\right]\right\}$ can be extended to a related family of hypersurfaces defined for $t$-values in a larger interval $\left[0, T_{0}+\varepsilon\right), \varepsilon>0$, which contradicts the definition of the value $T_{0}$. This contradiction proves the assertion.

For each $t \in\left[0, T_{0}\right)$ fixed, consider the related foliation of $W$

$$
\mathcal{F}(t)=\left\{\phi_{s}(\Lambda(t)) \mid s \in \mathbb{R}\right\},
$$

all whose leaves have constant mean curvature $-t$, see Fig. 10 left. Let

$$
L(t)=\phi_{S(t)}(\Lambda(t))
$$

be the unique leaf of $\mathcal{F}(t)$ such that $L(t)$ intersects $\Lambda(0)$ and lies on the lower side of $\Lambda(0)$. Assertion 9.3 implies that $s(t) \rightarrow-\infty$ as $t \rightarrow T_{0}$. Let $L\left(T_{0}\right)$ be the limit of the hypersurfaces $L(t)$ as $t \nearrow T_{0}$. Such a limit exists and it is a complete graphical hypersurface with constant mean curvature $-T_{0}$ by the stability of $L(t)$ for all $t<T_{0}$, and the same arguments as in the proof of Assertion 9.3. Let $\Lambda^{\prime}$ be the open subdomain of $\Lambda$ over which $L\left(T_{0}\right)$ is a $K_{1}$-graph. Clearly, $L\left(T_{0}\right)$ intersects $\Lambda(0)$ and lies below $\Lambda(0)$. Define $t_{0} \in \mathbb{R}$ 

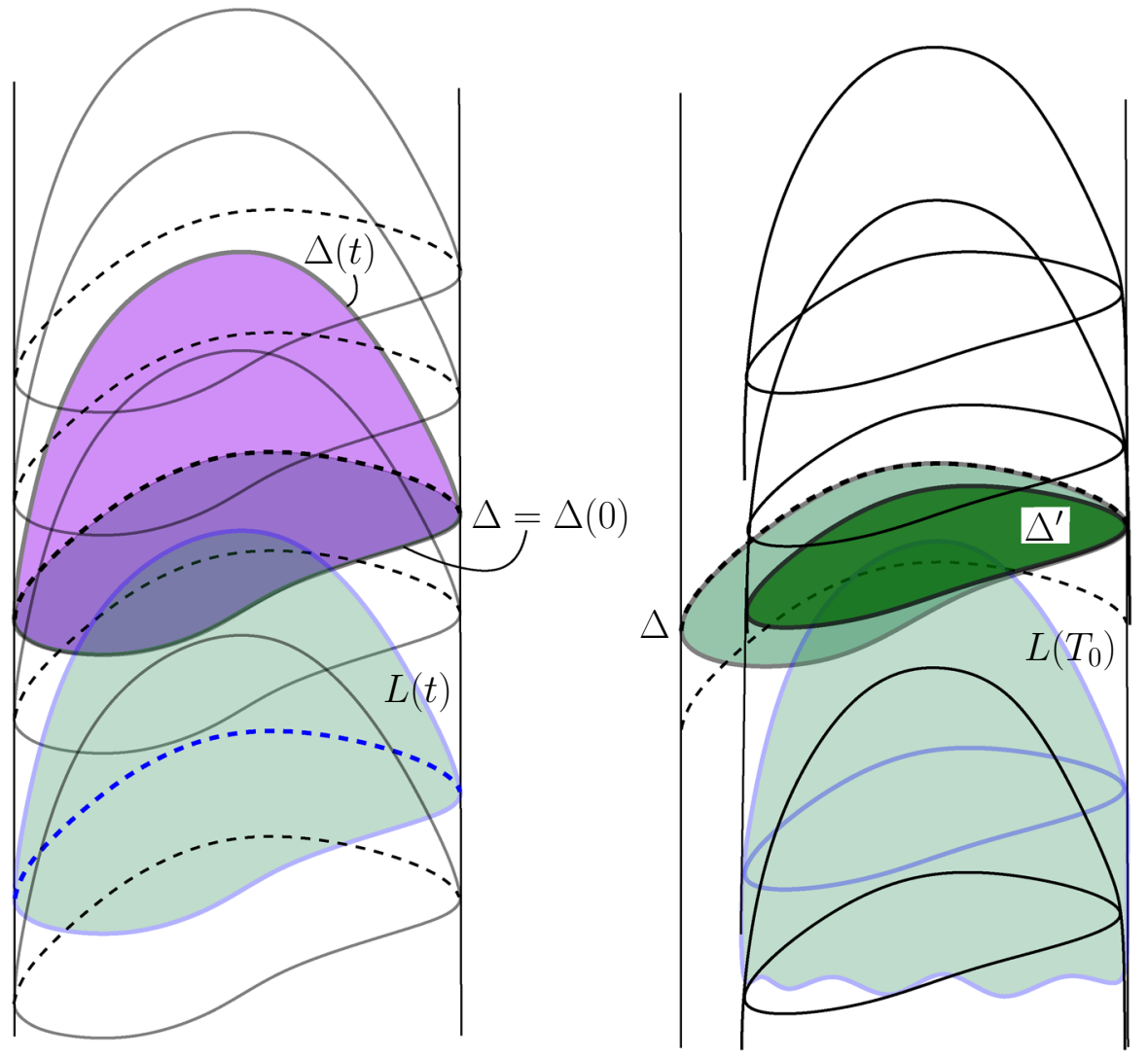

Fig. 10 Left: After blowing a bubble $\Lambda(t)$ with constant mean curvature $-t \in\left(-T_{0}, 0\right]$ (which is $K_{1}$-graphical over $\Lambda=\Lambda(0)$ ), we translate it with $\left\{\phi_{s}\right\}_{s \in \mathbb{R}}$ to produce a foliation $\mathcal{F}(t)$, and we highlight the highest graphical leaf $L(t)$ of $\mathcal{F}(t)$ lying below $\Lambda(0)$ with $L(t) \cap \Lambda(0) \neq \emptyset$. Right: Since the $\Lambda(t)$ with $t \rightarrow T_{0}^{-}$do not lie in a fixed compact set, we produce a noncompact limit $L\left(T_{0}\right)=\lim _{t \rightarrow T_{0}^{-}} L(t)$ which is a graph over a domain $\Lambda^{\prime}$ of $\Lambda$

such that $L\left(T_{0}\right)$ lies in $\Sigma \times\left(-\infty, t_{0}\right]$ and $t_{0}$ is the smallest value with this property. Observe that $L\left(T_{0}\right)$ intersects $\Sigma \times\left\{t_{0}\right\}$ tangentially at some interior point of $\Sigma \times\left\{t_{0}\right\}$.

Assertion $9.4 L\left(T_{0}\right)$ has linear volume growth. In particular, $L\left(T_{0}\right)$ is parabolic.

Proof of the assertion. A straightforward limit argument shows that $L\left(T_{0}\right)$ minimizes the functional Volume ${ }_{n}+n T_{0}$ Volume $_{n+1}$ on compact subsets of $\operatorname{Int}(W)$. This property implies that $L\left(T_{0}\right)$ has bounded second fundamental form. Therefore, the gradient of the graphing function $h: \Lambda^{\prime} \rightarrow \mathbb{R}$ that produces $L\left(T_{0}\right)$ as a $K_{1}$-graph becomes unbounded as we approach any point of the boundary of $\Lambda^{\prime}$ (otherwise we could enlarge $\Lambda^{\prime}$ ). Therefore the asser- 
tion holds provided that we check that the total $(n-1)$-dimensional volume function of the submanifold $\Gamma_{s}:=L\left(T_{0}\right) \cap \phi_{s}(\Lambda(0))$ of $L\left(T_{0}\right)$ is bounded as $s \rightarrow-\infty$. To see this, for $s$ large $(s \ll-1)$ let us denote by $\Omega(s)$ the portion of $L\left(T_{0}\right)$ lying above $\phi_{s}(\Lambda(0))$, and let $\eta_{s}$ be the outward pointing unit conormal vector field of $\Omega(s)$ along its boundary $\Gamma_{s}$. Note that for every $\varepsilon>0$ small, there exists $s_{0}(\varepsilon) \ll-1$ such that for each $s \leq s_{0}$,

$$
\left\langle K_{1}, \eta_{s}\right\rangle<-\varepsilon \text { along } \Gamma_{s},
$$

for some $\varepsilon>0$ independent of $s$. On the other hand,

$$
\operatorname{Flux}\left(\Omega(s), \Gamma_{s}, K_{1}\right)=\int_{\Gamma_{s}}\left\langle K_{1}, \eta_{s}\right\rangle+n T_{0} \int_{\phi_{s}\left(\Lambda_{s}\right)}\left\langle K_{1}, N_{\phi_{s}\left(\Lambda_{s}\right)}\right\rangle
$$

where $\Lambda_{s}$ is the subdomain of $\Lambda^{\prime}$ over which $\Omega(s)$ is a $K_{1}$-graph, and $N_{\phi_{s}\left(\Lambda_{s}\right)}=\left(\phi_{s}\right)_{*}\left(N_{\Lambda(0)}\right)$. By homological invariance of the flux, we have Flux $\left(\Omega(s), \Gamma_{s}, K_{1}\right)=0$. As the second integral in the right-hand-side of (9.7) is bounded above in absolute value by $\left|K_{1}\right|_{\infty}$ times the $n$-dimensional volume of $\Lambda(0)$ (here $\left|K_{1}\right|_{\infty}=\max _{\Lambda}\left|K_{1}\right|$, which exists since $\Lambda$ is compact and $K_{1}$ is generated by $\left\{\phi_{s} \mid s \in \mathbb{R}\right\}$ ), then the first integral in the right-hand-side of (9.7) is bounded independently of $s \rightarrow-\infty$. This property together with (9.6) ensure that the $(n-1)$-dimensional volume of $\Gamma_{s}$ is bounded as $s \rightarrow-\infty$. Now the assertion is proved.

Recall that by assumption, $K_{2}$ is bounded in $\Sigma \times(-\infty, 0]$. Define $u=$ $\left\langle K_{2}, N_{L\left(T_{0}\right)}\right\rangle: L\left(T_{0}\right) \rightarrow \mathbb{R}$, where $N_{L\left(T_{0}\right)}$ denotes a unit normal vector field along $L\left(T_{0}\right) . u$ is a bounded Jacobi function on $L\left(T_{0}\right)$, which vanishes at each of the points of the nonempty set $L\left(T_{0}\right) \cap\left(\Sigma \times\left\{t_{0}\right\}\right)$. Since every integral curve of $K_{2}$ that intersects $L\left(T_{0}\right)$ is a compact interval with its boundary in $\partial W$, then $K_{2}$ is not everywhere tangent to $L\left(T_{0}\right)$, which implies $u$ is not identically zero; by the maximum principle, $u$ must change sign in a neighborhood of any of the points in $L\left(T_{0}\right) \cap\left(\Sigma \times\left\{t_{0}\right\}\right)$. As $L\left(T_{0}\right)$ is complete, stable, and is parabolic by Assertion 9.4, we conclude that every bounded nonzero Jacobi function on $L\left(T_{0}\right)$ has constant sign [21, Corollary 1], which is a contradiction. This contradiction finishes the proof of Theorem 9.1.

\section{References}

1. Abresch, U., Rosenberg, H.: A Hopf differential for constant mean curvature surfaces in $\mathbb{S}^{2} \times \mathbb{R}$ and $\mathbb{H}^{2} \times \mathbb{R}$. Acta Math. 193(2), 141-174 (2004)

2. Abresch, U., Rosenberg, H.: Generalized Hopf differentials. Mat. Contemp. 28, 1-28 (2005)

3. Aronszajn, N.: A unique continuation theorem for solutions of elliptic partial differential equations or inequalities of second order. J. Math. Pures Appl. 36, 235-249 (1957) 
4. Bers, L.: Local behavior of solutions of general linear elliptic equations. Commun. Pure Appl. Math. 8, 473-496 (1955)

5. Cheng, S.Y.: Eigenfunctions and nodal sets. Comment. Math. Helv. 51(1), 43-55 (1976)

6. Chern, S.S.: On the minimal immersions of the two-sphere in a space of constant curvature. In: Problems in analysis (Lectures at the symposium in honor of Salomon Bochner, Princeton Univ., Princeton, 1969), pp. 27-40. Princeton Univ. Press, Princeton (1970)

7. Chern, S.S.: On surfaces of constant mean curvature in a three-dimensional space of constant curvature. In: Palis, J. (ed.) Geometric Dynamics. Lecture Notes in Mathematics, vol. 1007, pp. 104-108. Springer, Berlin (1983)

8. Daniel, B.: Isometric immersions into 3-dimensional homogeneous manifolds. Comment. Math. Helv. 82(1), 87-131 (2007)

9. Daniel, B., Hauswirth, L., Mira, P.: Constant Mean Curvature Surfaces in Homogeneous Manifolds. Korea Institute for Advanced Study, Seoul (2009)

10. Daniel, B., Mira, P.: Existence and uniqueness of constant mean curvature spheres in $\mathrm{Sol}_{3}$. Crelle J. Reine Angew. Math. 685, 1-32 (2013)

11. Fernández, I., Mira, P.: Constant mean curvature surfaces in 3-dimensional Thurston geometries. In: Proceedings of the International Congress of Mathematicians, Volume II (Invited Conferences), pp. 830-861. Hindustan Book Agency, New Delhi (2010). http:// arXiv.org/abs/1004.4752

12. Figueroa, C.B., Mercuri, F., Pedrosa, R.H.L.: Invariant surfaces of the Heisenberg groups. Ann. Mat. Pura Appl. 4(177), 173-194 (1999)

13. Gálvez, J.A., Mira, P.: Uniqueness of immersed spheres in three-manifolds. J. Differ. Geom. (to appear)

14. Hardt, R., Simon, L.: Boundary regularity and embedded minimal solutions for the oriented Plateau problem. Ann. Math. 110, 439-486 (1979)

15. Hoffman, D., de Lira, J.H.S., Rosenberg, H.: Constant mean curvature surfaces in $M^{2} \times \mathbb{R}$. Trans. Am. Math. Soc. 358(2), 491-507 (2006)

16. Hopf, H.: Differential Geometry in the Large, Volume 1000 of Lecture Notes in Mathematics. Springer, Berlin (1989)

17. Hsiang, W.T., Hsiang, W.Y.: On the uniqueness of isoperimetric solutions and imbedded soap bubbles in noncompact symmetric spaces. I. Invent. Math. 98(1), 39-58 (1989)

18. Jellet, J.H.: Sur la surface dont la coubure moyenne est constante. J. Math. Pures Appl. 18, $163-167$ (1853)

19. Korevaar, N., Kusner, R., Meeks III, W.H., Solomon, B.: Constant mean curvature surfaces in hyperbolic space. Am. J. Math. 114, 1-43 (1992)

20. Korevaar, N., Kusner, R., Solomon, B.: The structure of complete embedded surfaces with constant mean curvature. J. Differ. Geom. 30, 465-503 (1989)

21. Manzano, J.M., Pérez, J., Rodríguez, M.M.: Parabolic stable surfaces with constant mean curvature. Calc. Var. Partial Differ. Equ. 42, 137-152 (2011)

22. Mazet, L.: Cylindrically bounded constant mean curvature surfaces in $\mathbb{H}^{2} \times \mathbb{R}$. Trans. Am. Math. Soc. 367, 5329-5354 (2015)

23. Meeks III, W.H.: Constant mean curvature spheres in Sol3. Am. J. Math. 135(3), 763-775 (2013)

24. Meeks III, W.H., Mira, P., Pérez, J.: Embeddedness of spheres in homogeneous threemanifolds. Int. Math. Res. Not. 15, 4796-4813 (2017)

25. Meeks III, W.H., Mira, P., Pérez, J.: The geometry of stable minimal surfaces in metric Lie groups. Trans. Am. Math. Soc. 372(2), 1023-1056 (2019)

26. Meeks III, W.H., Mira, P., Pérez, J., Ros, A.: Constant mean curvature spheres in homogeneous three-spheres. J. Differ. Geom. (to appear)

27. Meeks III, W.H., Mira, P., Pérez, J., Ros, A.: Isoperimetric domains of large volume in homogeneous three-manifolds. Adv. Math. 264, 546-592 (2014) 
28. Meeks III, W.H., Pérez, J.: Constant mean curvature surfaces in metric Lie groups. In: Galvez, J., Pérez, J. (eds.) Geometric Analysis, vol. 570, pp. 25-110. Contemporary Mathematics, Singapore (2012)

29. Meeks III, W.H., Pérez, J., Ros, A.: Stable constant mean curvature surfaces. In: Ji, Lizhen, Li, Peter, Schoen, Richard, Simon, Leon (eds.) Handbook of Geometrical Analysis, vol. 1, pp. 301-380. International Press, Vienna (2008). ISBN: 978-1-57146-130-8

30. Meeks III, W.H., Tinaglia, G.: Existence of regular neighborhoods for $H$-surfaces. Ill. J. Math. 55(3), 835-844 (2011)

31. Meeks III, W.H., Yau, S.T.: Topology of three-dimensional manifolds and the embedding problems in minimal surface theory. Ann. Math. 112, 441-484 (1980)

32. Meeks III, W.H., Yau, S.T.: The classical Plateau problem and the topology of threedimensional manifolds. Topology 21(4), 409-442 (1982)

33. Meeks III, W.H., Yau, S.T.: The existence of embedded minimal surfaces and the problem of uniqueness. Math. Z. 179, 151-168 (1982)

34. Milnor, J.W.: Curvatures of left invariant metrics on Lie groups. Adv. Math. 21, 293-329 (1976)

35. Morrey, C.B.: Multiple Integrals in the Calculus of Variations. Springer, Berlin (1987)

36. Pedrosa, R., Ritoré, M.: Isoperimetric domains in the Riemannian product of a circle with a simply connected space form and applications to free boundary value problems. Indiana Univ. Math. J. 48, 1357-1394 (1999)

37. Ros, A.: One-sided complete stable minimal surfaces. J. Differ. Geom. 74, 69-92 (2006)

38. Schoen, R.: Estimates for Stable Minimal Surfaces in Three Dimensional Manifolds, volume 103 of Annals of Mathematics Studies. Princeton University Press, Princeton (1983)

39. Scott, P.: The geometries of 3-manifolds. Bull. Lond. Math. Soc. 15(5), 401-487 (1983)

40. Sekigawa, K.: On some 3-dimensional curvature homogeneous spaces. Tensor (N.S.) 31(1), 87-97 (1977)

41. Souam, R.: On stable constant mean curvature surfaces in $\mathbb{S}^{2} \times \mathbb{R}$ and $\mathbb{H}^{2} \times \mathbb{R}$. Trans. Am. Math. Soc. 362(6), 2845-2857 (2010)

42. Torralbo, F.: Rotationally invariant constant mean curvature surfaces in homogeneous 3manifolds. Differ. Geom. Appl. 28, 523-607 (2010)

Publisher's Note Springer Nature remains neutral with regard to jurisdictional claims in published maps and institutional affiliations. 